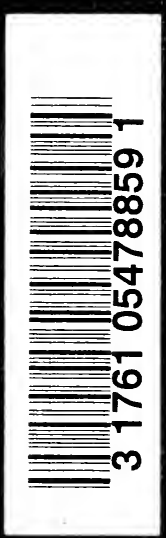


Digitized by the Internet Archive in 2007 with funding from Microsoft Corporation 


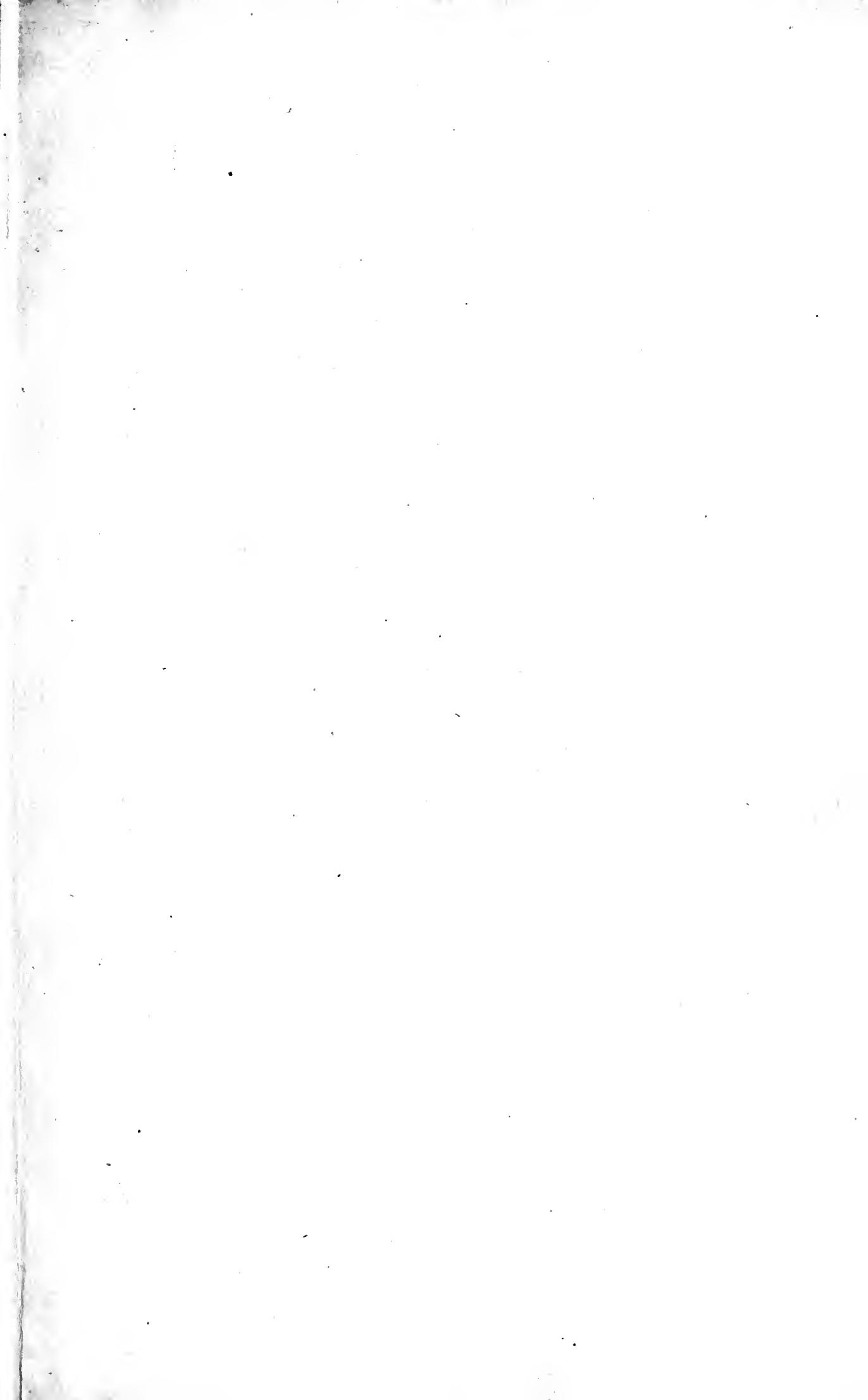





\title{
AN INTRODUCTION TO THERMODYNAMICS
}

\section{FOR ENGINEERING STUDENTS}

\author{
BI \\ JOHN MILLS \\ PROFESSOR OF PHYSICS AND ELECTRICAL ENGINEERING \\ COLORADO COLLEGE
}

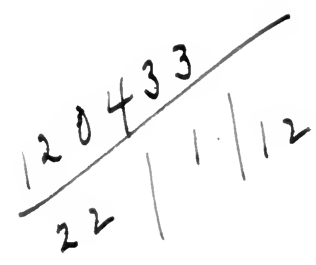

GINN AND COMPANY

BOSTON · NEW YORK - CHICAGO - LONDON 
Copyright, 1910

BY JOHN MILLS

ALL RIGHTS RESERVED

710.9

Cbe atbenxum Dress

GIN ANI) COMPANY PKO-

PKIETUKS - BOSTUN - U.S.A. 


\section{PREFACE}

This book is the outcome of the writer's desire to put into the hands of a class, studying introductory thermodynamics, in a concise form a statement of those principles and concepts which are essential to the study of the construction and operation of steam engines, steam turbines, and their auxiliaries, and, to a more limited extent, of air compressors and gas engines. The book deals only with the ideal and limiting cases, and aims only at a preparation of the student for reading the more advanced technical works or papers. As such the text makes no claim to originality except, to some extent, in the selection and arrangement of material and in the location of the emphasis.

The material selected is from such sources as the works and books of Clausius, Rainkine, Carnot, Kelvin, Stodola, Pellat, Planck, Preston, Edser, Bryan, Boulvin, Swinburne, Peabody, and Buckingham. The arrangement is intended to lead the student from the sophomore physies work into thermodynamics as a continuation and more detailed study of a portion of physical science already somewhat familiar. The emphasis is therefore placed upon the physical concepts, and constant reference is made to the molecular kinetic theory, which is usually familiar to such a student. Although an effort has been made to eliminate as far as practicable the use and solution of differential equations, it is hoped that should the book be used by a student who later takes up the study of pure thermodynamics, he will have little if anything to unlearn and will find the physical interpretation of the mathematical relations rendered easier. The emphasis is also placed upon the solution of numerical problems, many examples of which are given. These in general are arranged to lead from the simpler concepts to the ideal limiting cases of the applications to engineering problems. 
In addition to the general statement of sources made above, acknowlerlgments of details are made in footnotes. It is the writer's pleasure to acknowledge the influenee of former study under Professor (. W. Berry of the Massachusetts Institute of Technology. To the inspiration of Professor R. A. Millikan of the University of Chicago, a friend and former teacher, who has read a portion of the proof of this text, a general acknowledgment is gratefully made for most that is good in the writer's point of view and pedagogical method in physics.

Colorado Springs, Colorado 


\section{CONTENTS}

\section{CHAPTER I. FUNDAMENTAL CONCEPTS AND LAWS}

1. Work. 2. Energy. 3. Molecular Kinetic Theory. 4. Temperature. 5. Thermal Units. 6. First Law of Thermodynamics. 7. Specific Heat. 8. Component Parts of Specific Heat. 9. Latent Heat. 10. Boyle's Law. 11. Joule's Law. 12. Perfect Gases. 13. Charles's Law. 14. Standard Hydrogen Thermometer. 15. Absolute Temperature. 16. General Equation for a Perfect Gas. 17. Use of the Term "Specific." 18. Specific Heat of Gases. 19. Graphical Representation of Gas Transformations. 20. Work Calculations for Gas Transformations. 21. Adiabatic Transformation. 22. Comparison of Adiabatic and Isothermal Transformations. 23. Second Law of Thermodynamics. 24. Carnot Cycle. 25. Reversible Cycle. 26. Carnot Theorem. 27. Discussion of Reversible Processes. 28. Discussion of the Second Law. 29. Thermodynamic Scale of Temperature. 30. Carnot Cycle for a Perfect Gas. 31. Entropy. 32. Measurement of Entropy. 33. Temperature-Entropy Plot. 34. Discussion of Carnot Cycle from $\theta-\phi$ Plot. 35. Entropy Changes in Irreversible Processes.

Chapter II. GaSES . . . . . . . . . . . . . . . .

36. Graphical Representation of State for a Perfect Gas. 37. Equations for Polytropic Transformation for a Perfect Gas. 38. Fundamental Heat Equations for a Perfect Gas. 39. Entropy Changes for a Perfect Gas. 40. Relation of the Specific Heats of a Gas. 41. Changes in Intrinsic Energy of a Gas. 42. Imperfect Gases. 43. Résumé of Equations of Chapters I and II.

Problems and Solutions: Gases. . . . . . . . . . . . . .

1. Use of the Characteristic Equation. 2. Carnot Cycle. 3. Intrinsic Energy. 4. Constant-Pressure Transformation. 5. Exponent $n$. 6. Entropy Changes. 7. Isothermal Expansion. 8. Adiabatic Expansion. 9. Simultaneous Equations in $p$ and $v$. 10. Pressure-Volume Plot. 11. Regenerative Cycle, Sterling Engine. 12. Air Compressor with and without Clearance. 13. Otto Gas Engine Cycle. 14. Diesel Internal Combustion Cycle. 15. Air Refrigeration.

CHAPTER III. WATER AND ITS SATURATED VAPOR. . . . .

44. Phenomena of Vaporization. 45. Specific Heat of Water. 46. Heat of Vaporization. 47. Quality of Steam. 48. Intrinsic Energy of Steam. 49. Entropy of the Liquid. 50. Entropy of Vaporization. 51. Temperature-Entropy Chart for Steam. 52. Isentropic Transformation of a Mixture. 53. Specific Heat of Steam. 54. Résumé of Equations for a Mixture of Steam and Water.

Problems and Solutions: Saturated Water Vapor

16. Quality. 17. Intrinsic Energy. 18. Isoenergic Transformation. 19. Isothermal Transformation. 20. Adiabatic Transformation. 21. Constant-Volume Transformation. 22. Boiler Explosion. 23. Equivalent Evaporation and Boiler 
Horse Power. 24. Surface Condenser. 25. Jet Condenser. 26. Rankine Cycle. 27. Incomplete Expansion. 28. Efficiency and Boiler Pressure. 29. Condensing Engine. 30. Lse of the Temperature-Entropy Chart. 31. Boulvin Diagram. 32. Hyperbolic Expansion.

CHAPTER IY. SEPERHEATED STEAM . . . . . . . . . .

5.5. Three Molecular States. 56. Superheated Steam. 57. Specific Heat of Superheated Steam. 58. Entropy of Superheated Steam. 59. TemperatureEntropy Chart for Superheated Steam. 60. Intrinsic Energy of Superheated Steam. 61. Isentropic 'Transformation. 62. Superheating in Engineering Practice. 63. Résumé of Equations for Superheated Steam.

Problems axd Solltioxs: Superheated Steay . . . . . . . . .

33. Specific Volume. 34. Temperature of Superheat. 35. Specific Heat of Superheated Steam. 36. Total Heat. 37. Entropy. 38. Quality. 39. Quality after Adiabatic Expansion. 40. Intrinsic Energy. 41. Work of Adiabatic Expansion. 42. Gain by Superheating.

\section{CHAPTER V. FLOW OF STEAM AND GASES}

64. Formula of de Saint-Venant. 65. Alternative Expression for the Formula of de Saint-Venant. 66. Zeuner's Formula for Kinetic Energy. 67. Kinetic Energy of an Irreversible Adiabatic Expansion. 68. Loss of Kinetic Energy due to Friction. 69. Flow through an Orifice. 70. Fliegner's Formulas for the Flow of Air. 71. Résumé of Equations for the Flow of Fluids.

Problems and Solutions : Flow of Fluids . . . . . . . . . . . .

43. Kinetic Energy of Steam. 44. Kinetic Energy of Superheated Steam. 45. Loss due to Friction. 46. Flow of Air. 47 and 48. Napier's Formula. 49. Nonlifting Injector. 50. Peabody Throttling Calorimeter. 51. Limits of the Throttling Calorimeter. 52. Throttle Control. 53. Nozzle Area. 54. Turbine Nozzle.

MISCELLANEOLS PROBLEMS . . • . . . . . • • • • • • • 125

TABLES . . . . . . . . . . . . . . . . . . . . 129

1 and 2. Gas Constants. 3. Steam and Water Constants. 4. Logarithms. 


\section{SYMBOLS}

The symbols giver below are used consistently throughout this text. They have been selected, as far as is possible, in accordance with the following rules: (1) a given symbol is to be used for but one physical magnitude, (2) a lower-case letter is always to represent the value per unit mass of the magnitude to which it refers, (3) the symbols are to be in accordance with the American usage.

$A=1 / J=$ factor for reducing mechanical units of energy to heat units.

$a=$ general expression for area.

$\alpha=$ volume coefficient of a gas

$$
=\frac{1}{V}\left(\frac{d V}{d t}\right)_{p}
$$

$A p u=$ external latent heat of vaporization in thermal units.

B.t. $u .=$ British thermal unit.

$\beta=$ pressure coefficient of a gas

$c=$ general expression for specific heat.

$c_{p}=$ specific heat at constant pressure.

$c_{v}=$ specific heat at constant volume.

$E=$ total intrinsic energy of a substance.

$\epsilon=$ intrinsic energy of a unit mass of a substance.

$\eta=$ thermal efficiency of a process or cycle.

$\boldsymbol{F}=$ general expression for force.

$g=$ numerical value of the acceleration due to gravity.

$\gamma=$ general expression for density.

$h=$ heat units required to superheat from dry saturated steam, per unit mass.

$I=$ intrinsic energy due to molecular configuration.

$J=$ factor for reducing thermal units to work units.

$\kappa=$ ratio of $c_{p}$ to $c_{v}$.

$l=$ loss of kinetic energy due to friction, per unit mass of steam. $\lambda=$ total heat contents per unit mass of a mixture (from $32^{\circ} \mathrm{F}$.), e.g. $\lambda=q+r+h$, or $\lambda=q+x r$, or $\lambda=q+x \rho+x A p u$.

$M=$ mass (or weight) per second in flow of fluids.

$m=$ mass (or weight).

$n=$ general expression for the exponent in the relation $p v^{n}=$ constant.

$p=$ general expression for pressure (i.e. specific pressure).

$\phi=$ general expression for entropy.

$\phi_{w}=$ general expression for entropy of liquid (per unit mass) above that of water at $32^{\circ} \mathrm{F}$.

$\phi_{v}=$ increase in entropy per unit mass due to vaporization.

$\phi_{s}=$ increase in entropy per unit mass due to superheating.

$q=$ heat contents of water per unit mass measured from $32^{\circ} \mathrm{F}$.

$\mathfrak{Q}=$ general expression for heat transferred to or from an external source.

$\mathfrak{q}=$ general expression for heat transferred per unit mass.

$R=$ constant in the gas relation

$$
p V=m R \theta .
$$

$r=$ total latent heat of vaporization per unit mass.

$\rho=$ internal latent heat of vaporization per unit mass.

$S=$ intrinsic energy due to molecular motion.

$s=$ specific volume of dry saturated steam.

$\sigma=$ specific volume of water $=0.016$ cubic foot per pound. 
$T=$ temperature measured on the thermodynamic scale.

$t=$ general expression for temperature.

$\mathbf{t}=$ general expression for time.

$\theta=$ absolute temperature as measured on a perfect gas scale.

$t_{0}=$ saturation temperature of dry steam before superheating.

$\theta_{0}=$ absolute saturation temperature of dry steam before superheating.

$V=$ general expression for volume.

$v=$ general expression for the volume of a mixture per unit mass.

$\mathfrak{v}=$ velocity. $u=$ increase in specific volume due to vaporization, i.e. $u=s-\sigma$.

$W=$ general expression for external mechanical work.

$w=$ general expression for external work per unit mass of working substance.

$x=$ quality of a mixture of $x$ parts steam and $(1-x)$ parts water.

$y=$ friction work during flow through a channel per unit mass of the fluid. $z$ and $d z=$ distance through which a force acts, e.g. $W=\int F d z$. 


\section{AN INTRODUCTION TO THERMODYNAMICS}

\section{CHAPTER I \\ FUNDAMENTAL CONCEPTS AND LAWS}

1. Work. Work is defined as the act of overcoming a resistance through space. It is measured by the product of the component of the acting force along the direction of the motion of its point of application, and the distance through which its point of application moves during its action. The scholium to Newton's 'Third Law of Motion states that "if the action (activity) of an agent be measured by its amount and its velocity conjointly, and if similarly the reaction (counter-activity) of the resistance be measured by the velocities of its several parts and their amounts conjointly, whether these arise from friction, molecular force, weight, or acceleration, action and reaction in all combinations of machines will be equal and opposite." Since velocity is merely the rate at which space is traversed, it is seen that this scholium states that during any given time the work of the acting forces is equal to the work of the resisting forces.

The unit of work depends upon the chosen unit of force. In the absolute C. G. S. system where the unit of force is the dyne (a force which if acting constantly upon one gram will produce a change in its velocity of one centimeter per second every second), the unit of work is the erg, or the work done by one dyne acting through one centimeter. In the gravitational C. G. S. system the unit of force is the gram weight (i.e. 980 dynes), and the unit of work is the gramcentimeter. The force unit in the F.P.S. system (gravitational) is the pound, or the force of the earth's attraction upon one pound of matter, and the unit of work is the foot pound.

2. Energy. Energy is defined as the capacity of a body for doing work. It is evident, then, that work and energy are to be measured in the same units. In general the energy possessed by a body or by a system of bodies may be considered as the result of work done upon that body or system by another body or system. The energy of a system may be either its capacity to do work as the result of 
work done upon the system in giving to its component parts motion (that is, work done against the resistance of acceleration), in which case it is known as kinetic energy; or, it may be the result of work done upon the system in producing the existing configuration of its component parts (that is, work done against gravitation or other forces), in which case it is known as potential energy.

The sum of the potential and kinetic energy of a body is commonly called its intrinsic energy. The Principle of the Conservation of Energy states that if work is done upon a body the amount of work is equal to the increase in intrinsic energy of the body; that is, to the sum of the increases in the kinetic and potential energy. And conversely, the work done by a body is equal to the decrease in its intrinsic energy. Thus, if $d \boldsymbol{E}$ represents the increase in the intrinsic energy of a body and $d W$ the amount of work done by the body, then $d E+d W=0$.

The measure of the kinetic energy of a rigid body is the sum of its kinetic energy of translation and its kinetic energy of rotation. The kinetic energy of translation is $\frac{1}{2} m \mathfrak{v}_{0}^{2}$, where $m$ is the mass of the body and $\mathfrak{b}_{0}$ is its absolute velocity of translation. The kinetic energy of rotation is $\frac{1}{2} K \omega_{0}^{2}$, where $K$ is the moment of inertia of the body about an axis through its center of mass, and $\omega_{0}$ is the absolute velocity of rotation about this axis. The potential energy of a body is $\int F d z$, where the integral represents the work done against the resisting forces in moving the body from a position of zero potential energy to the position under consideration.

From the above statement of the measure of the intrinsic energy of a body it is evident that its energy is the total amount of work which could be derived from it under the most favorable theoretical considerations. For the practical purposes of the universe in which we live the intrinsic energy may be considered in two parts; namely, available and unavailable energy. The availability of the intrinsic energy of a body depends upon external conditions, as will be seen later in various problems. A simple mechanical illustration is as follows. Consider the velocity of a cannon ball of mass $m$ to be $\mathfrak{v}_{0}^{2}$, then its energy is $\frac{1}{2} m \mathfrak{v}_{0}^{2}$. If the velocity of the ball after it has struck a ship initially at rest (that is, the velocity communicated to the ship by the impact) be represented by $\mathfrak{v}$, then the intrinsic energy of the ball is now $\frac{1}{2} m \mathfrak{b}^{2}$. Of the total energy of the ball only the portion $\frac{1}{2} m\left(\mathfrak{v}_{0}^{2}-\mathfrak{v}^{2}\right)$ was available for the destruction of the ship. 
3. Molecular Kinetic Theory. The molecular kinetic theory is based upon certain assumptions as to the character and actions of molecules which do not pretend to represent the actual character of the molecules but which simplify the problem so that it admits of a mathematical analysis. The molecules of a gas (for which state many of the assumptions would be approximately correct) are considered to be in motion, to act like hard, smooth, small, and perfectly elastic spheres, exerting no forces upon each other except at the instant of collision, and to exist in almost immeasurable numbers in any finite volume of the gas. These assumptions admit of the application of the laws of probability, and of the treatment of collisions as if the molecules were uniform spheres of unit coefficient of restitution, colliding centrally.

Upon these assumptions by a mathematical analysis Maxwell, Clausius, Joule, Boltzmann, and others have developed conclusions ${ }^{1}$ as to the behavior of a gas which agree with the observed facts as expressed in the well-known laws of Boyle, Charles, Dalton (and Avogadro).

As expressed by Risteen, one of these conclusions is as follows: "In a molecular mixture there is one physical quality which is the same for every set of molecules; and that is, the average kinetic energy of translation per molecule." Now it is an observed fact that whenever two or more substances are brought into intimate contact there is one physical property which is the same for all of the substances, namely, the temperature. Hence, it is natural to assume that equality in the temperature means equality in energy of translation of the molecules.

4. Temperature. The temperature of a body is merely. an arbitrary expression of its "hotness" as compared with some states of hotness assumed as standard. Because, however, of the inexactness of the sense of touch as a measure of the "hotness" and because of the experimentally determined fact that some substances expand as they become hotter, the expansion or contraction of a given amount of such a substance is arbitrarily taken as the quantitative measure of a temperature change. For ordinary purposes the substance used is mercury enclosed in glass, and the relative expansion of the mercury is taken as a measure of the change in temperature.

\footnotetext{
1 It is desirable at this point to give merely the briefest possible résumé of the kinetic theory (the facts of which are already familiar to the student from his course in general physics), in order that the concept of temperature may be approached from the side of energy. For a more complete statement in non-mathematical language see Risteen, " Molecules and the Molecular Theory," pp. 42-43.
} 
The scale upon which temperature is measured has been chosen in a purely arbitrary manner. On the Centigrade scale one degree ${ }^{1}$ of temperature represents a change in temperature equal to that which would produce one one-hundredth of the effect produced in the mercury thermometer by a difference in temperature corresponding to the following easily reproducible states of pure water. The lower point, called zero degrees, is the temperature of pure water and melting ice in an intimate mixture. The upper point, called one hundred degrees, is the temperature of the vapor of pure boiling water in contact with its liquid. In both cases (although it is of primary importance only in the second case) the pressure upon the mixture must be what is known as standard or normal pressure, which is equal to that exerted by a column of mercury 76 centimeters high, when the mercury is at the temperature of melting ice and water. The scale is extended in each direction from these two fixed points by divisions corresponding to a degree as defined alove.

The Fahrenheit scale has 180 degrees which are equivalent to 100 degrees on the Centigrade scale. The fixed point corresponding to the temperature of melting ice and water is marked 32 degrees, and the boiling point is 212 degrees.

According to the molecular kinetic theory two bodies are at the same temperature when the average kinetic energy of translation of their molecules is the same. It will be shown later in Section 15 that according to this theory one degree Centigrade is a difference in temperature such that the average kinetic energy of translation of the molecules of the substance considered, is increased (or diminished) by one one-hundredth of the change in this kinetic energy which would be produced by a difference in temperature corresponding to that between zero and one hundred degrees Centigrade. It will also be seen that this is, for a gaseous body, approximately equal to $\frac{1}{2} \overline{7}$ of the average kinetic energy of the molecules when the substance is at zero degrees Centigrade.

5. Thermal Units. When two bodies at different temperatures are placed in proximity, it is found that they ultimately come to a common temperature (unless this difference in temperature is maintained by some external agency). According to the kinetic theory this must mean that a transfer of energy has taken place from one borly to the other in order that the final average molecular energy of translation of the two bodies shall be the same. Energy trans-

\footnotetext{
1 For more rigorous definition of a degree Centigrade see Section 14.
} 
ferred under these conditions is known as heat. Because it was not recognized in the earlier development of physical science that heat is energy, units were introduced for the measurement of heat.

These thermal units are essentially arbitrary and relative, since they depend upon the unit of temperature difference. Thus the heat (energy) required to change the temperature of one gram of pure water from $15^{\circ}$ to $16^{\circ}$ Centigrade is the thermal unit in the C. G. S. system and is called the calorie. And similarly the amount of heat (energy) required to raise the temperature of one pound of water from $62^{\circ}$ to $63^{\circ} \mathrm{Fahrenheit}$ is called a British thermal unit, or as abbreviated a B.t.u.

6. First Law of Thermodynamics. The Principle of the Conservation of Energy was not established until the classical work of Joule (and Mayer) performed during the years following 1843. This principle may be stated in a more general form than that given in Section 2 as follows: "Every physical or chemical change of state has a fixed mechanical equivalent expressible in work units." Thus, for example, the amount of heat (or energy) required to produce in water the change in physical state which is produced by a calorie (or a B.t.u.) is to be equated under all conditions to a definite number of work units. This particular application of the Principle of the Conservation of Energy was experimentally shown to be correct by Joule and was really the basis for the adoption of the principle in its broadest form. This application has received the name of the First Law of Thermodynamics. As usually stated it reads: "Heat and mechanical work are mutually convertible, ${ }^{1}$ and heat requires for its production and produces by its disappearance a certain definite amount of work for each thermal unit." The value of this mechanical equivalent of heat from the determination made by Rowlands is 778 foot pounds per B.t.u., or $4.19 \times 10^{7}$ ergs per calorie. In symbols this law may be written

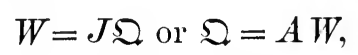

where $\mathfrak{Q}$ is the number of thermal units, $W$ the number of mechanical units which correspond, and $J$ is the (mechanical) equivalent of each thermal unit in work units for the system in which $W$ is to be expressed. The factor $A$ is evidently the reciprocal of $J$.

7. Specific Heat. The amount of heat required to produce a change of one degree in the temperature of a substance depends

1 Except in so far as all the heat energy of a body is not available for such a conversion, as is discussed in Section 2 and also in later sections. 
upon the nature of the substance, the mass in which it is desired to produce this change, and, as will be seen in Section 18, upon the conditions under which the change is produced. For the same mass of a given substance it is found that the amount of heat required per degree is different at different temperatures; that is, it is a function of the temperature. The thermal capacity per unit mass or "specific heat" of a substance is defined as the number of units of heat required to change the temperature of unit mass of the substance one degree. This is expressed either in calories per gram or in B.t.u. per pound, and the numbers representing the value of the specific heat of a given substance are obviously the same in the two systems. But it would be better to express the specific heat directly in mechanical units, since these belong to an absolute system and since this may readily be done in accordance with the First Law of Thermodynamics. Thus $J$, the mechanical equivalent of heat, is merely the specific heat of water in absolute units.

Since the specific heat of a substance is slightly different at different temperatures, it may best be defined by a differential notation. Thus if a quantity of heat $d \mathfrak{q}$ be added to a unit mass of a substance and produce an increase in the temperature of $d t$, then the specific heat $c$, at the temperature $t$, at which the addition was made, or the heat required per degree per unit mass, is $\frac{d \mathfrak{q}}{d t}=c$. Hence the total heat $\mathfrak{\Omega}$ required to produce in a mass $m$ a change of temperature from $t_{1}$ to $t_{2}$ is

$$
\mathfrak{Q}=m \int_{t_{1}}^{t_{2}} d t . . . \quad \text {. . . . }
$$

Frequently, it is sufficiently accurate to assume the specific heat constant through the range of temperature $t_{1}$ to $t_{2}$ and to write

$$
\mathfrak{Q}=m c\left(t_{2}-t_{1}\right),
$$

where $c$ is the average value of the specific heat of the substance for a range of temperature which includes $t_{1}$ and $t_{2}$.

8. Component Parts of Specific Heat. When the temperature of any substance is changed by the addition of a quantity of heat, it is possible to consider the change as composed of three parts, and it is frequently possible to assign to each part its proper portion of the heat energy transferred. In general, heat added to a substance may be considered as producing first, a change in the temperature; second, a change in the configuration of the molecules composing the substance; and third, because of the consequent increase (or decrease) 
of the volume occupied by the substance, a certain amount of external work. The first portion, which goes to produce a change in the temperature, increases according to the molecular theory the average kinetic energy of translation of the molecules. ${ }^{1}$ This portion will be represented by the symbol $A d S$. 'The second portion of the heat supplied produces a change in the potential energy of the molecules and will be represented by $A d I$. The third portion, representing an amount of external work, will be symbolized by $d W$. In general, then, if a small amount of heat $d \mathfrak{Q}$ is required to produce a change in the temperature of a substance, we may write

$$
d \mathfrak{Q}=A(d S+d I+d W), . \quad . \quad . \quad .
$$

where $d S, d I$, and $d W$ are all expressed in mechanical units.

In the case of the change of state from water to steam, to be discussed later, the term $d I$ is large as compared with the other two terms. In the case of a "perfect gas," also to be discussed later, the term $d I$ is zero for all changes.

9. Latent Heat. In the case of a change of state, as that referred to above, where water is converted into a vapor, or vice versa (or in the case of the formation of ice from water, or vice versa) there is produced no change in temperature until sufficient heat energy has been added, or subtracted, to produce the change in potential energy for the entire mass corresponding to the different configuration of the molecules. During such a change of state the term $A d S$ is then zero. The term $\frac{A d I}{m}$, representing the amount of heat required per unit mass to produce the change in internal potential energy, is known as the internal latent heat for that particular change of state. This internal latent heat is also a function of the temperature at which the change of state occurs, as will be discussed more fully later, in the case of steam. As commonly expressed the latent heat is the sum of the terms $\frac{A d I}{m}$ and $\frac{A d W}{m}$, the latter representing the external latent heat or external work which must be done in producing the change.

Thus in C. G. S. units, the latent heat of vaporization of water at $100^{\circ}$ Centigrade and under an atmospheric pressure of 76 centi-

\footnotetext{
1 It is true also that an increase in temperature means in general an increase in the kinetic energy of rotation of the molecule and also an increase in the "sub-molecular" kinetic and potential energy of the component parts of the molecule, but for the further purposes of this discussion all this energy will be considered as included by the term AdS.
} 
meters of mercury is 536.5 calories. Of this the part corresponding to $\frac{A d W}{m}$ may be found as follows. The volume occupied by one gram of water changes from one cubic centimeter to 1649 cubic centimeters, which is the volume occupied by one gram of water vapor under the given condition of temperature. Since this change takes place against a pressure of (76) (13.6) (980) dynes per square centimeter, $(76)(13.6)(980)(1649-1)$, or $1.668 \times 10^{9}$, ergs represent the external work during evaporation. That is, the external latent heat of evaporation is $1.668 \times 10^{9} \div 4.19 \times 10^{7}=40$ calories for the conditions of the problem. The internal latent heat, representing the gain in molecular potential energy, is then 496.5 calories or $2.08 \times 10^{10} \mathrm{ergs}$.

10. Boyle's Law. Boyle's Law states that, other things being equal (that is, the temperature, mass, and molecular constitution of the gas experimented upon remaining unchanged), the product of the pressure at which a given gas is maintained and the volume which it occupies is a constant. Or, in other words, if the pressure is changed, the volume occupied by a given quantity of gas maintained at a constant temperature undergoes a change inversely proportional to the change in pressure. In symbols this is expressed as $p V=$ a constant, or

$$
p_{1} V_{1}=p_{2} V_{2}=p_{3} V_{3}, \cdot \text {. . . . } .
$$

where $p$ represents the pressure or force per unit area and $V$ represents the volume (usually measured in the same system of units as is the area).

The value of the constant is evidently for a given mass of some particular gas a function of the temperature. This follows at once from the observable fact that if the temperature is changed, the value of the product $p V$ is also changed. The physical meaning of this fact is evident from the following analysis based upon the assumptions of the kinetic theory.

The pressure upon the bounding surface of a body of gas is due to the impacts of the moving molecules. It is proportional to the number of these impacts per second upon each unit area of the bounding surface, and to the impulse of each molecular impact. The impulse is proportional to the change in momentum of the moving molecule, and this in turn to the velocity and mass of the molecule. The total number of impacts made by a given molecule will be proportional to its velocity. The number of molecular impacts upon each unit of area of the bounding surface will be proportional 
to the gas density ; that is, for a given mass of gas, inversely proportional to the entire volume. Hence the pressure is directly proportional to the mass of a molecule and to the square of the average molecular velocity, and inversely proportional to the entire volume. That is, the product of the pressure and the volume is directly proportional to the average molecular kinetic energy of translation, and hence is some function of the temperature. ${ }^{1}$

This law of Boyle is not rigorously correct, for all gases show departures from it at high pressures. It is followed very closely for pressures under 10 atmospheres. For higher pressures the departures are more marked. Thus for air they range from about $\frac{1}{4}$ per cent at 10 atmospheres to as much as 25 per cent at 600 atmospheres.

11. Joule's Law. Another important law of gases is due to experiments performed by Joule. This law states that "all the work done in compressing a gas is conducted away as heat if the temperature of the gas is kept constant, and conversely, when a gas expands at constant temperature, it must receive from its surroundings a quantity of heat equal to the work done by the expansion." This means that during the expansion of a gas no internal work is done by the molecules either by molecular repulsions or against molecular attractions. For if there are attractive forces between the molecules, then in such an expansion, since the molecules become more widely separated, work must be done to produce this separation, and this must be supplied by the partial conversion of molecular kinetic energy into molecular potential energy. If there is to be no consequent lowering in the temperature, there must be supplied to the molecules of the substance from the outside an amount of heat energy equal to that converted into molecular potential energy. The amount of heat supplied to an expanding gas would then be greater than the amount of external work performed by the gas during expansion by this amount of increase in the molecular potential energy. According to Joule's Law there are then no molecular attractive forces acting between molecules. On the other hand, if repellent forces exist between the molecules, the potential energy term will decrease in value, and there will be a larger amount of external work done than there is heat supplied to the expanding gas. Thus, the possibility of repellent forces between the molecules of a gas is also denied by Joule's Law. In so far, then, as a

1 See any.general physics text, or Millikan, "Mechanics, Molecular Physies, and Heat," Chapters XIV and XVI. 
gas obeys Joule's Law the potential energy term $d I$ in the equation $d \mathfrak{Z}=A(d S+d I+d W)$ must be zero. This law is not more rigorous than Boyle's Law, and the departures from Boyle's Law may be explained in part upon the assumption that there are molecular attractions which are not entirely negligible when the density is high and the molecules close together.

Joule's classical experiment was performed with apparatus shown diagrammatically in Figure 1. Two similar metal vessels $A$ and $B$,

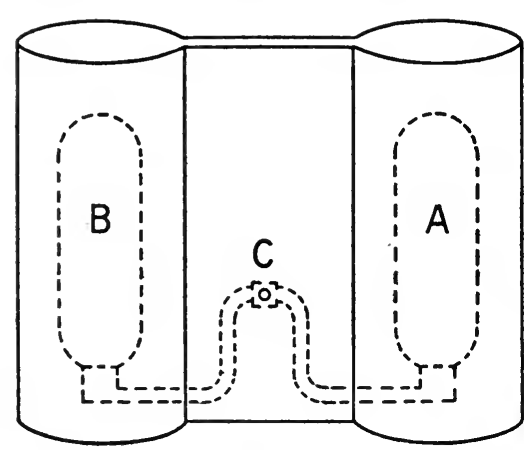

FIG. 1 connecting by a tube fitted with a stopcock $C$, were enclosed in a large calorimeter. The temperature was measured by a thermometer reading to $0^{\circ} .005$ Fahrenheit. ${ }^{1}$ The vessel $A$ contained air under a pressure of about 22 atmospheres, and the vessel $B$ was practically a vacuum. When the stopcock was opened, the air in $A$ expanded into $B$ without doing external work. It was found impossible to detect any change in the temperature of the water as a result of this expansion. From this experiment Joule concluded that "no change in temperature occurs when air is allowed to expand in such a manner as not to develop mechanical work." From this there follows the conclusions stated above as to the intrinsic potential energy of the molecules of a gas.

12. Perfect Gases. Since all gases show departures from the approximate laws of Boyle and Joule, but since these departures are not in general large, it is convenient for the purposes of analysis in problems dealing with gases to treat of purely fictitious so-called "perfect gases." A perfect gas is defined as one for which the laws of Boyle and Joule hold rigorously for all pressures and temperatures. Such a gas would then have no interacting forces between its molecules except at the instant of mutual impact, and according

1 The more accurate "porous plug experiment" of Kelvin and Joule is described later (see Section 35), but merely as an illustration of an entropy change in an irreversible adiabatic expansion. The conelusions of that experiment which bear upon this discussion are here stated. At orlinary temperatures there is a small but appreeiable attraction between the molecules of a gas, and hence a cooling by free expansion through an orifice. The cooling is ronghly $0^{\circ} .25$ ) Centigrale per atmosphere of pressure difference on the two sides of the orifice. The value is less for higher temperatures, and greater for lower temperatures. This phenomenon, sncressively caused to take place in the same mass of gas, is of course the basis of liquil air machines. 
to the kinetic theory (see Section 10, above) the product of the pressure and the volume would be some function of the temperature.

Writing $p V=f(t)$, where $f(t)$ represents some function of the temperature, it is possible to show that for a perfect gas the values of the "pressure coefficient" and of the "volume coefficient" are equal when determined for the same temperature. The pressure coefficient $\beta$ is defined as the increase in pressure per degree of temperature per unit of pressure. In symbols this is expressed as

$$
\beta=\frac{p_{1}-p_{0}}{p_{0}\left(t_{1}-t_{0}\right)}
$$

where $p_{0}$ is the original pressure exerted by a given mass of gas at a temperature of $t_{0}$, and $p_{1}$ and $t_{1}$ represent the pressure and temperature of some later condition of the gas while occupying the same -volume. In differential notation this is most conveniently expressed by using the symbol ()$_{V}$ to indicate the fact that the quantity enclosed by the parenthesis is found under the conditions of constant volume. Thus

$$
\beta=\frac{1}{p}\left(\frac{d p}{d t}\right)_{\nabla} \cdot \cdot \cdot \cdot \cdot \cdot \cdot \cdot .
$$

Similarly, the expansion coefficient $\alpha$ is

$$
\alpha=\frac{1}{V}\left(\frac{d V}{d t}\right)_{p} \cdot \cdot \cdot \cdot \cdot \cdot \cdot \cdot \cdot \cdot
$$

From the expression of Boyle's Law for a perfect gas as $p V=f(t)$ we have

$$
\begin{aligned}
& \beta=\frac{1}{p}\left(\frac{d f(t)}{V d t}\right)_{V}=\frac{f^{\prime}(t)}{p V}=\frac{f^{\prime}(t)}{f(t)}, \\
& \alpha=\frac{1}{V}\left(\frac{d V}{d t}\right)_{p}=\frac{f^{\prime}(t)}{p V}=\frac{f^{\prime}(t)}{f(t)} .
\end{aligned}
$$

The values of $\alpha$ and of $\beta$ are evidently equal for the same values of the temperature $t$.

The fact that all gases have the same value of $\alpha$ for any given temperature was discovered as an experimental fact by Charles in 1787, some sixty years before the announcement of Joule's Law, which has been used here in the definition of a perfect gas.

13. Charles's Law. Charles's Law states that all gases show the same fractional or per cent expansions between the limits of two fixed temperatures. Later experiments have shown that this law of Charles (or of Gay-Lussac, as it is sometimes known) is only 
approximate. Experiments have also shown that for all gases the values of the pressure coefficient $\beta$ for the same initial temperature are practically equal, and are also practically equivalent in value to the values of the expansion coefficient $\alpha$ for the same temperature. This is evident from Table $1,{ }^{1}$ where are given for various gases values of $\alpha$ and $\beta$ which represent their average values for the range of temperature from $0^{\circ}$ to $100^{\circ}$ Centigrade.

The definition of a perfect gas leads then to the conclusion that for perfect gases Charles's Law holds rigorously, and also the values of $\alpha$ and $\beta$ are identical. For a perfect gas the value of $\beta$ will be taken as 0.0036625 at $0^{\circ}$ Centigrade, as is explained in Section 15.

14. Standard Hydrogen Thermometer. Because of the fact that the expansion of most solids and liquids is not a linear function of the temperature, while that of a gas is very nearly a linear function in accordance with Charles's Law, the standard thermometric substance is a gas. A gas thermometer might, obviously, with almost equal justification, be constructed to measure temperature by the expansion of the gas under the condition of constant pressure, or by the change in pressure under the condition of constant volume. Because of the experimental difficulty of making the corrections which enter into a determination of temperature with a constant pressure gas thermometer (due to the variations in the exposed length of the column of gas contained in the stem of the thermometer), Regnault adopted the constant volume form. In 1887 the International Committee of Weights and Measures adopted the constant volume hydrogen thermometer as the standard. According to this agreement one degree Centigrade is such a temperature as will cause a change in the pressure of a mass of hydrogen (kept at a constant volume) of 0.0036625 , or $\frac{1}{2} \frac{1}{3}$, of the pressure which this hydrogen exerts at the temperature of melting ice and pure water.

15. Absolute Temperature. According to the discussion of Section 10 for a constant volume perfect gas thermometer we may write the pressure as proportional to the average molecular kinetic energy of translation. If, further, the pressure coefficient $\beta$ be taken for a perfect gas as equal to the value 0.0036625 , whch is given by Chappuis for hydrogen, a gas which most nearly obeys the laws of perfect gases, it may be shown, as follows, that one degree Centigrade change in the temperature of a perfect gas corresponds to a change in the average molecular kinetic energy of $\frac{1}{2} \overline{3}$ part of the 
value at zero degrees Centigrade of this average molecular kinetic energy. Thus

\section{Hence}

$$
p V \propto \text { av. K. E. }
$$

$$
\left(\frac{d p}{d t}\right)_{V} \propto\left(\frac{d(\text { av. K. E. })}{d t}\right)_{V}
$$

and

or

$$
\alpha=\beta=\frac{1}{p}\left(\frac{d p}{d t}\right)_{V}=\frac{1}{\text { av. K. E. }}\left(\frac{d(\text { av. K. E. })}{d t}\right)_{V},
$$

$$
\frac{1}{273}=\frac{1}{\text { av. K. E. at } 0^{\circ} \mathrm{C} .}\left(\frac{d(\text { av. K. E. })}{d t}\right)_{V \text { at } 0^{\circ} \mathrm{C} .,} .
$$

where the symbol " ( $)_{V}$ at $0^{\circ} \mathrm{C}$." is used to represent the fact that the volume is maintained constant at the value which it has for a temperature of zero degrees Centigrade.

If, then, the temperature of a perfect gas be lowered to $-273^{\circ}$ Centigrade, it follows that the molecules would be completely deprived of kinetic energy of translation, and hence at rest. The pressure exerted by the gas would then be zero. This temperature of $-273^{\circ}$ Centigrade is known as the absolute zero; for, if temperatures are measured in degrees absolute, that is in degrees from this absolute zero, the intrinsic energy of a perfect gas is directly proportional to its temperature. For all practical problems the intrinsic energy of the so-called permanent gases may also be taken to be proportional to the absolute temperature.

This absolute zero is not an attainable temperature. All known gases liquefy and even solidify above this temperature. Thus hydrogen liquefies at about $-252^{\circ} \mathrm{C}$. and solidifies at about $-256^{\circ} \mathrm{C}$. By evaporating liquid helium a temperature of about $-270^{\circ} .5 \mathrm{C}$. has been attained. These low temperatures are measured with a platinum resistance thermometer which has been found to give results very close to that of a hydrogen thermometer.

For a perfect gas, and for most practical problems in gases, under the conditions of constant volume the pressure may be taken proportional to the absolute temperature. This is expressed in symbols in equation (9). And similarly, for constant pressure, the volume may be taken proportional to the absolute temperature, as is expressed in symbols in equation (10)

$$
\begin{aligned}
& \left(\frac{p_{2}}{p_{1}}\right)_{V}=\left(\frac{\theta_{2}}{\theta_{1}}\right)_{V}, \ldots . . \\
& \left(\frac{V_{2}}{V_{1}}\right)_{p}=\left(\frac{\theta_{2}}{\theta_{1}}\right)_{p}, . \quad . \quad . \quad . \quad . \quad . \quad .
\end{aligned}
$$


where $\theta$ is the absolute temperature as measured on the perfect gas scale. Or, in symbols,

$$
\theta_{0}=273 . \quad \theta_{1}=t_{1}+273 \text { in degrees Centigrade. . . . . }
$$

And since $273^{\circ}$ Centigrade equals $491^{\circ} .5$ Fahrenheit,

$$
\theta_{0}=459.5 . \quad \theta_{1}=t_{1}+459.5 \text { in degrees Fahrenheit. . . . }
$$

16. General Equation for a Perfect Gas. It is evident since

$$
p V^{\top} \propto \text { average molecular K. E. } \propto \theta,
$$

that a general expression for the pressure, volume, and absolute temperature of a perfect gas may be written as follows:

$$
p V=m R \theta, .
$$

where $p$ is pressure, $V$ is volume, $\theta$ is the absolute temperature, $m$ is the mass of the gas, and $R$ is a constant. The value of $R$ depends upon the kind of gas and has a numerical value which represents in the system of units to which $m$ belongs the value of the expression $\frac{p v}{\theta}$ for unit mass of the gas for any condition for which the corresponding values of $p, v$, and $\theta$ are known.

Thus for air, assuming that it will follow the equation for a perfect gas, and finding $R$ for the C.G.S. system, we have the density of air at the standard conditions of 76 centimeters of mercury pressure and zero degrees Centigrade equal to 0.001293 as given by Regnault for latitude $45^{\circ}$ (where $g$ is 980.6 ). The volume of one gram of air for these conditions is then 773.4 cubic centimeters. The value of $p$ is $1.0134 \times 10^{6}$ dynes per square centimeter. The absolute temperature, $\theta$, is 273 . Hence $R$ is $2.867 \times 10^{6}$.

Similarly for the F.P.S. system, the volume of one pound of air is 12.39 enbic feet at a pressure of 14.7 pounds per square inch and at a temperature of $32^{\circ}$ Fahrenheit. Expressing the pressure $p$ as $144 \times 14.7$ pounds per square foot and substituting, gives $R$ equal to 53.35 .

17. Use of the Term "Specific." As used in physics the meaning of the term "specific" may best be shown by illustration. Thus "specific heat" is the amount of heat required to produce a definite result, namely, unit change of temperature in unit mass. The term "sprecifie" then indicates on the part of the physical magnitude to which it is applied a definite and peculiar quality in which this particular value of the magnitude differs from all other possible values of the same magnitude. In general it limits the magnitude to unit 
mass, unit length, unit area, unit volume, or to a unit change in some related magnitude. Thus "specific resistance" is the resistance per unit cube, or the resistance per unit length having unit cross section, as in the case of the mil-foot.

The adjective "specific" is somewhat more widely used in engineering, where the terminology is not otherwise as carefully differentiated as in pure physics. Thus in the rigorous physical usage "pressure" always means "force per unit area," but in engineering usage, broadened and rendered less precise by the influence of the necessity of conveying ideas in popular language, pressure and force are used almost interchangeably. For this reason there has come into common engineering use the term "specific pressure" to mean force per unit area, as for example in the F.P.S. system, where specific pressure is the force in pounds per square foot. Similarly, "density," which to the student of physics means "mass per unit volume," is expressed by the engineer as "specific weight," meaning thereby the weight per cubic foot. "Specific volume" is the volume of one pound in cubic feet. "Specific gravity" is the weight of a given volume of a substance in terms of the weight of the same volume of water, taken as unity. 1

For the purposes of this book the word "pressure" will always be used in its rigorous sense of force per unit area. Also, the following scheme of notation has been adopted to avoid confusion. Whenever volume, quantity of heat, or any other unit which may later be introduced refers to unit mass of the substance under consideration, a lower case letter will be used. Whenever reference is made to the total value of a magnitude, referring to some other mass than unity, a capital letter will be used. It will be noticed that this has been done consistently in the earlier portion of the text; thus, $p V=m R \theta$, but $p v=R \theta$.

18. Specific Heat of Gases. The specific heat of a gas may be determined under the condition either of constant volume or of constant pressure. It is evident from a consideration of equation (4) of Section 8 that when the volume is constant, since the term $d W$ is zero (because there is no external work done in expanding),

1 This inexactitude of common engineering terminology has frequent illustration. Thus "speed" and "velocity" are used interchangeably. "Acceleration," which is the rate of change of velocity, is popularized into " rate of acceleration," an expression which rigorously is a second derivative of velocity instead of the first. "Electromotive force" and "potential difference" are frequently usel as synonymous. Also "energy" and "power" are not carefully distinguished, as is illustrated by the frequent use of expressious like " expenditure of power." 
the amount of heat required to produce in unit mass a temperature change of one degree is less than that required to produce this change when the pressure is kept constant and the term $d W$ has a numerical value (representing the work done externally by the expansion of the gas against the constant pressure to which it is subjected). These two values of the specific heat of a gas will in the future be represented by $c_{p}$ and $c_{v}$ for the conditions of constant pressure and constant volume, respectively. Or, in symbols,

$$
c_{p}=\left(\frac{d \mathfrak{q}}{d t}\right)_{p} \text { and } c_{v}=\left(\frac{d \mathfrak{q}}{d t}\right)_{v} \cdot \text {. . . . . . }
$$

The ratio of the specific heats of a gas (written $\left.\kappa=\frac{c_{p}}{c_{v}}\right)$ may be shown to depend upon the atomic structure ${ }^{1}$ of the gas considered. For monatomic gases this ratio $\kappa$ has a value of 1.66 , for diatomic gases a value of about 1.40; for the diatomic mixture constituting air the value is 1.401 , for carbon dioxide it is 1.30 (at $30^{\circ} \mathrm{C}$.).

19. Graphical Representation of Gas Transformations. Changes in the values of the magnitudes $p, V$, and $\theta$ for a given mass of gas may best be represented graphically by plotting on a set of coördinate axes values of $V$ as abseissas and values of $p$ as ordinates. Since the temperature $\theta$ is a function of $p$ and $V$, it is implicitly represented upon the same plot.

The possible ways in which a given mass of gas may undergo a continuous transformation, not involving any chemical change, are evidently the following : (a) at constant volume by changing the pressure indirectly through a change in the temperature; $(b)$ at constant pressure by changing the volume indirectly through a change in the temperature; (c) at constant temperature, that is isothermally, and hence in accordance with the relation of Boyle's Law; the values of $p$ and $V$ are then related as $p V=p_{1} V_{1}=$ a constant; $(d)$ without the addition or subtraction of heat, that is, as it is called, adiabatically; as will be seen in Section 21 the equation expressing this relation is $p V^{\kappa}=p_{1} V^{\kappa}{ }_{1}=$ a constant; (e) in accordance with the condition that the intrinsie energy shall be constant, that is, an isoenergic transformation; for a perfect gas this condition is evidently equivalent to that of an isothermal change; $(f)$ the change may be under the condition of constancy in what is called the entropy of the gas, that is, the change may be isentropic; for a reversible transformation, as will be discussed in Section 31, this is equivalent to

1 See Edser, "Heat for Advanced Students," p. 302. 
an adiabatic change; or $(g)$ the transformation may not be in accord with any of the above conditions, in which case frequently it may be represented by a relation of the form $p V^{n}=p_{1} V_{1}^{n}=$ a constant; or, it may be assumed to take place in a series of infinitesimal steps which are in accordance with some of the above conditions.

The plots of Figure 2, lettered $a, b, c, d$, and $g$, represent graphically changes in accordance with the conditions of $a, b, c, d$, and $g$,

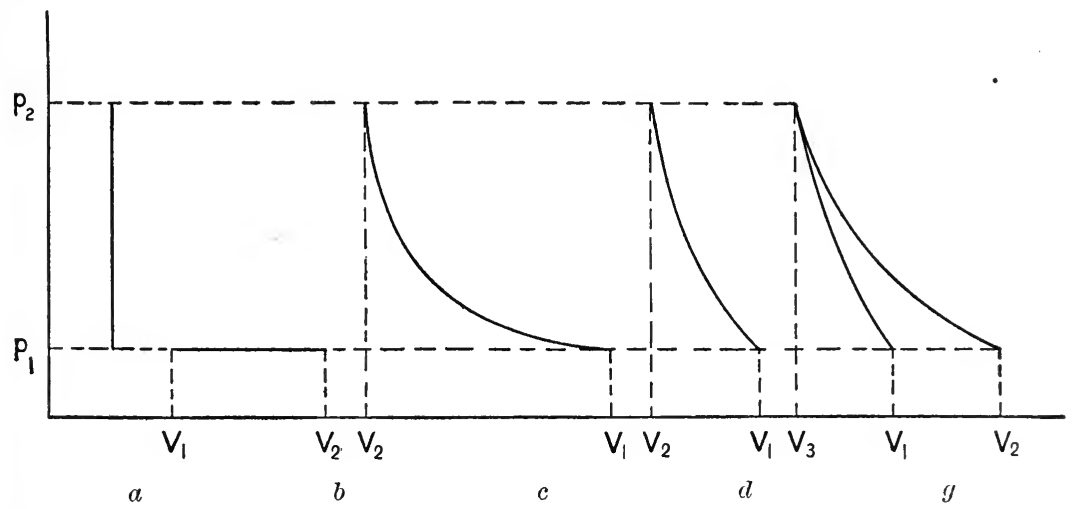

Fig. 2

above, from a volume and pressure of $V_{1}$ and $p_{1}$ to a volume and pressure of $V_{2}$ and $p_{2}$, respectively. The plot $(g)$ is drawn to a very much larger scale than the others and represents disproportionately a change as taking place in two imaginary or component infinitesimal steps; namely, from $\left(p_{1}, V_{1}\right)$ to $\left(p_{2}, V_{3}\right)$ by an adiabatic compression and from $\left(p_{2}, V_{3}\right)$ to $\left(p_{1}, V_{2}\right)$ by an isothermal expansion.

20. Work Calculations for Gas Transformations. The work required to produce a change in the state of a gas, or the external work done by the gas, during some transformation, may be represented graphically upon the pressure-volume plot just described. Thus, consider that a quantity of gas is contained in a vessel fitted with a piston of area $a$. If the pressure of the gas is $p$ units of force per unit of area, then the total force exerted upon this piston is $F=p a$ units. If the piston is caused to move a small distance $d z$ by this force (or against it), the infinitesimal amount of work $d W$ is $d W=F d z=$ padz. During this motion the volume is changed by an infinitesimal amount $d V$, which is evidently equal to adz. The expression for the work done in producing this change is then $d W=F d z=p a d z=p d V$. This may be considered positive if the gas, or working substance, produces the change and does this amount 
of external work, and negative if the work is done by an external agent upon the working substance. The total work required to produce the change from a volume $V_{1}$ to a volume $V_{2}$ is then

$$
W=\int_{V_{1}}^{V_{2}} p d V
$$

If the summation is performed between the limits as shown above, it is evident that an expansion will be positive and correspond to work done by the substance, and similarly a compression will be negative and correspond to work done upon the substance.

The integration given above is only possible analytically when $p$ is either constant or some known function of $V$. In the " $p-V$ plot," however, the work required to produce a given change in volume is always to be represented by the area enclosed between the axis of volumes, the curve $p=f(V)$, and the two ordinates of values $V_{1}$ and $V_{2}$.

It is important to note that although the above discussion referred specifically to gases, the integral $\int p d V$ between the proper limits always gives the mechanical work required to produce the change in volume indicated. In the case of the transformations of steam in a steam engine the pressure-volume plot is drawn by a mechanism known as an indicator, which is attached to the cylinder of the engine in such a manner as to record pressures and to the piston of the engine so as to record piston displacements. The work done by the steam is then found approximately by using a planimeter to determine the area.

The cases mentioned in Section 19 and shown in Figure 2, $a, b, c$, and $d$, will now be discussed. For completeness, in addition to representing the value of the work integral there will be recorded at this time the energy relations and the heat relations for these changes, although some of these relations are not fully developed until Sections 21 and 22. The following symbols will be used: to represent intrinsic energy $\boldsymbol{E}$; to represent heat added to the substance from some external source $\mathfrak{Q}$. The subscripts 1 and 2 will represent states 1 and 2 respectively. It is to be noticed that the relations given below hold only for a perfect gas or one which may be assumed perfect, and in every instance of a change of volume the change is taken as an expansion; that is, $V_{2}$ is greater than $V_{1}$.

(a) Since the volume is constant, $W=0$. Because the temperature is increased the intrinsic energy is increased and by an amount

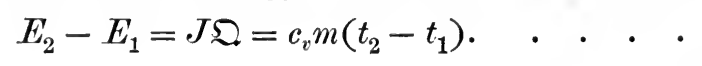


(b) Since $p$ is constant,

$$
\begin{aligned}
W & =p\left(T_{2}-T_{1}\right) . \quad \text { Also } \\
J \mathfrak{Q} & =c_{p} m\left(t_{2}-t_{1}\right), \\
& =W+E_{2}-E_{1} . \quad . \quad . \quad . \quad . \quad .
\end{aligned}
$$

(c) For an isothermal expansion

$$
\begin{gathered}
E_{2}-E_{1}=0, \text { and } \mathfrak{Q}=A W, \text { where } \\
W=\int_{V_{1}}^{V} p d V=\int_{V_{1}}^{V_{2}} p_{1} V_{1} \frac{d V}{V}=p_{1} V_{1} \log _{e} \frac{V_{2}}{V_{1}}=p_{1} V_{1} \log _{e} \frac{p_{1}}{p_{2}} \\
=m R \theta_{1} \log _{e} \frac{p_{1}}{p_{2}} .
\end{gathered}
$$

(d) For an adiabatic expansion $\mathfrak{Q}=0$, and $W=E_{2}-E_{2}$, where

$$
\begin{aligned}
W=\int_{V_{1}}^{V_{2}} p d V & =\int_{V_{1}}^{V_{2}} p_{1} V_{1}^{\kappa} \frac{d V}{V^{\kappa}}=\frac{p_{1} V_{1}}{\kappa-1}\left[1-\left(\frac{T_{1}}{V_{2}}\right)^{\kappa-1}\right] \\
& =\frac{p_{1} V_{1}}{\kappa-1}\left[1-\left(\frac{p_{2}}{p_{1}}\right)^{\frac{\kappa-1}{\kappa}}\right]=\frac{p_{1} V_{1}-p_{2} V_{2}}{\kappa-1} . . . .
\end{aligned}
$$

These integrations should be verified by the student. The results will be used in subsequent discussions.

21. Adiabatic Transformation. The equation stated in Section 19 as representing the relation for an adiabatic transformation of a gas will now be deduced. Consider a temperature change of infinitesimal amount taking place in a unit mass of the gas as the result of the addition of an infinitesimal amount of heat energy $\delta \mathfrak{q}$. Imagine the change to take place in two infinitesimal steps as follows, a change in temperature of amount $(\delta \theta)_{v}$ while the volume remains constant, and a change of $(\delta \theta)_{p}$ while the pressure remains constant. Since part of this change is at constant volume and part at constant pressure, the amount of heat $\delta q$ required to produce the change is the sum of the heat required to produce these component changes, namely, $c_{v}(\delta \theta)_{v}$ and $c_{p}(\delta \theta)_{p}$, where $c_{v}$ and $c_{p}$ are the specific heats at constant volume and at constant pressure respectively.

Thus

$$
\delta \mathfrak{q}=c_{v}(\delta \theta)_{v}+c_{p}(\delta \theta)_{p^{*}} \quad . \quad . \quad .
$$

Since, however, for this unit mass of gas $p$ and $v$ are related by the equation $p v=R \theta$, it follows that a change of temperature of amount $(\delta \theta)_{v}$ corresponds to and is expressible by $(v / R) \delta p$, and similarly a change of temperature $(\delta \theta)_{p}$ is expressible as $(p / R) \delta v$. Making 
this change of variables and writing in differential notation instead of the notation of infinitesimals, gives

$$
d q=c_{v} \frac{v}{R} d p+c_{p} \frac{p}{R} d v . \quad \text {. . . . . }
$$

Equation (21) is a general heat equation for a perfect gas and expresses the effect of adding heat energy in terms of the changes of the pressure and the volume of the gas. In Section 38 similar equations will be written expressing this effect in terms of the other possible variables of the perfect gas equation, namely, $p$ and $\theta$, and $v$ and $\theta$.

In the case of an adiabatic transformation, that is by definition one which takes place under conditions which prevent the transfer of heat to or from the substance, obviously the term $d \mathfrak{d}$ is zero, and equation (21) becomes

$$
c_{v} \frac{v}{R} d p+c_{p} \frac{p}{R} d v=0 . \quad \text {. . . . . . }
$$

Upon substitution of $\kappa=c_{p} / c_{v}$ (see definition of $\kappa$ in Section 18), this gives

$$
\kappa \frac{d v}{v}=-\frac{d p}{p}
$$

Solving this differential equation by integration between the limits of $p_{1}$ and $v_{1}$, which represents the condition of the gas in the first state, and $p_{2}$ and $v_{2}$, which represents its second condition, gives

$$
\log _{e}\left(\frac{v_{2}}{v_{1}}\right)^{\kappa}=\log _{e} \frac{p_{1}}{p_{2}}, \text {. . . . . . . }
$$

whence

$$
p v^{\kappa}=p_{1} v_{1}{ }^{\kappa}=p_{2} v_{2}{ }^{\kappa}, \quad \cdot \quad \cdot \quad \cdot
$$

or using the volume of $m$ units instead of one unit of mass,

$$
p_{1} V_{1}^{\kappa}=p_{2} V_{2}^{\kappa} \text {. }
$$

22. Comparison of Adiabatic and Isothermal Transformations. It is evident from equation (22) that the curve representing an adiabatic transformation on a pressure-volume plot is steeper than that representing an isothermal transformation, inasmuch as the tangent of the angle of slope, given by $d p / d v$ is $\kappa$ times as large for the adiabatic as for the isothermal curve. This is in aceordance with the physical idleas involved; for, considering a compression, it is clear that the work done by an external agent would tend to raise the temperature of the gas. If the change is isothermal, this must be prevented by conducting away the heat equivalent of the work 
as fast as it is produced. The isothermal change is represented in Figure 3 by $a b$. In an adiabatic compression, on the other liand, this is not done, and the heat goes into increasing the molecular motion of the gas. The adiabatic is $a c$ in Figure 3. This increased molecular motion means an increased pressure for the same final volume as was attained in the isothermal compression. Similar reasoning applies to the case of an expansion.

In general, it is to be noted that if a compression is adiabatic the work done goes into raising the

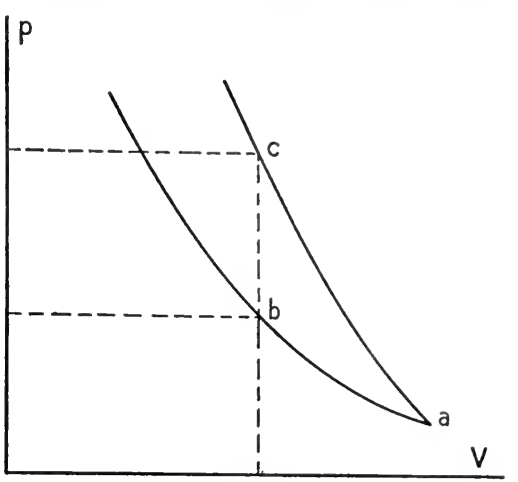

Fig. 3 temperature and increasing the intrinsic energy of the gas. If an expansion is adiabatic, part of the intrinsic energy of the gas is transformed into external work and the temperature of the gas is lowered. In both cases the amount of work done is numerically equal to the change in intrinsic energy. On the other hand, if the change is isothermal, heat energy must be given off during a compression and absorbed by the gas during an expansion, and the amount of this heat energy in the case of a perfect gas is equivalent to the work done, as is stated in Joule's Law. Thus, an isothermal transformation for most practical gas problems may be considered an isoenergic transformation.

23. Second Law of Thermodynamics. It was stated in Section 19 that for a reversible process an adiabatic transformation was also a so-called isentropic transformation. The concept of entropy may best be developed after a discussion of a cycle of changes known as the "Carnot cycle" and of a law known as the "Second Law of Thermodynamics."

The Second Law of Thermodynamics is similar to several laws of physics in that it admits of no direct verification and applies essentially to ideal cases. The first Newtonian law of motion is of this kind, for it states that "every body continues in a state of rest or of uniform motion in a straight line except in so far as compelled by force to change this state." The condition of a body free from the action of forces, either of friction or of gravitational attraction, is one that is not reproducible in nature, and yet the law is accepted as essentially axiomatic, because as nearly as the conditions can be 
produced the law is in accord with known facts. The Second Law of Thermodynamics may be accepted in somewhat the same manner, not because it is capable of direct verification but because no deduction from it that is capable of experimental proof has ever failed of verification.

The Second Law of Thermodynamics states that "it is impossible for any self-acting machine, unaided by any external agency, to convey heat from a body at a low temperature to one at a high temperature; or heat cannot of itself (that is, without the performance of work by some external agency) pass from a cold to a warmer body." This law applies only to a type of operations known as "cyclic." Its discussion will be postponed until after a description of the "cycle" of changes suggested by Carnot.

24. Carnot Cycle. Imagine a working substance, as for example air or gas, to be contained in a cylinder fitted with a piston. The
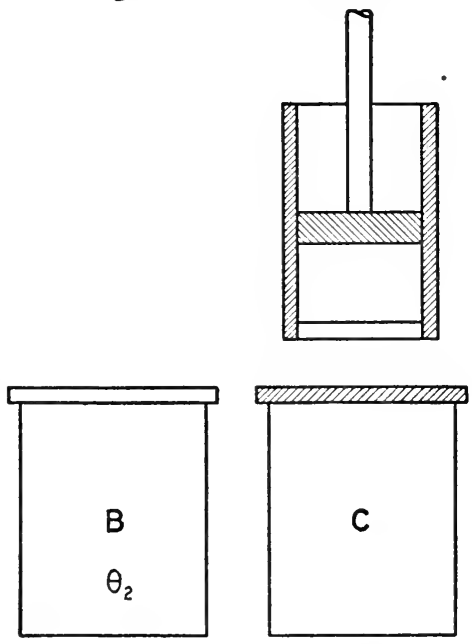

FIG. 4 sides of the cylinder and the piston are supposed to be absolute non-conductors of heat. But the bottom of the cylinder is assumed to be a perfect conductor of heat. Let there be imagined also two sources of heat energy, diagrammed as $A$ and $B$ in Figure 4, which are maintained at constant temperatures of $\theta_{1}$ and $\theta_{2}$, where $\theta_{1}$ is higher than $\theta_{2}$. Let there also be provided an insulating stand shown at $C$. Let the condition of the gas in the cylinder be represented by the point $a$ on the pressure-volume plot of Figure 5, and let this original condition be so chosen that the temperature is $\theta_{1}$.

Place the cylinder upon the hot source $A$ and allow the gas to expand isothermally from its initial state of $p_{1}, V_{1}, \theta_{1}$, to a state $p_{2}$, $V_{2}, \theta_{1}$, represented by the point $b$. During this expansion the energy necessary to do the external work against the pressure of the piston is derived from the source $A$. Now remove the cylinder 
from $A$ and placing it upon the insulating stand $C$, allow an adiabatic expansion until the temperature has fallen to that of the cold source $B$, namely, $\theta_{2}$. The point $c$ of coördinates $p_{3}$ and $V_{3}$ represents on the pressure-volume plot the state of the gas. Now place the cylinder upon the source $B$ and compress isothermally until the state of the gas is represented by $d$ of coördinates $p_{4}$ and $V_{4}$, and temperature $\theta_{2}$. The point $d$ is conditioned by the requirements that it shall lie upon an adiabatic through $a$ as well as upon the isothermal through $c$. Then removing the cylinder, place it upon the stand $C$ and compress the contents adia-

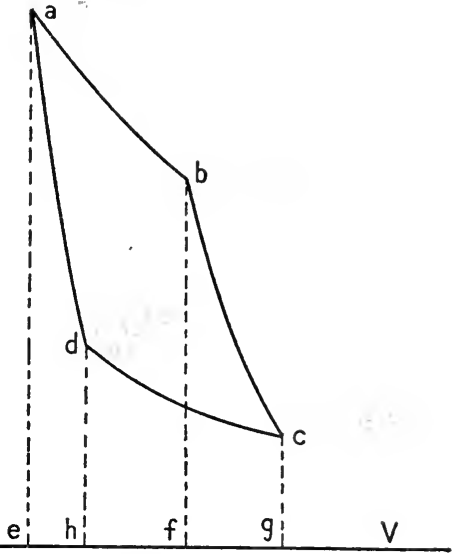

FIG. 5 batically until the temperature has risen to $\theta_{1}$, when the state of the gas will again be represented by the point $a$.

This set of four successive operations is called a cycle for the obvious reason that the working substance undergoes a series of changes which retirn it to its original condition. The cycle is known as the Carnot Cycle from its proposer, and the scheme here presented is known as a Carnot Engine. In this ideal heat engine the emphasis is placed upon the working substance and its cycle of changes, and not upon the mechanical details by which such an engine could be used practically.

These four operations will now be examined in more detail. During the isothermal expansion from $a$ to $b$ an amount of heat equal to $A \times$ area $a b f e$ has been received from the hot source and converted into work. During the adiabatic expansion from $b$ to $c$ an amount of intrinsic energy equal in heat units to $A \times$ area $b c g f$ has been converted into mechanical work. This has been at the expense of the temperature, as noted above. During the isothermal compression from $c$ to $d$ an amount of external work has been converted into $\boldsymbol{A} \times$ area $c d h g$ heat units and abstracted from the gas by the cold source $B$. During the adiabatic compression from $d$ to $a$ an amount of heat equal to $A \times$ area daeh has been given to the working substance as the result of the external work, and the temperature has 
consequently risen. The net result of the cycle of operations has been therefore the performance of $W$ units of work by the gas, where

$$
W=\text { area }(a b f e+b c g f-c g h d-d h e a)=\text { area } a b c d .
$$

Further, since the molecular energy possessed by the working substance is the same at any point on an isoenergic and hence isothermal line, it follows that the change produced in the intrinsic energy in passing from one isothermal to another is independent of the path followed. Hence, it is considered evident that the loss in intrinsic energy which occurs during the adiabatic expansion from $b$ to $c$ is numerically equal to the gain occasioned by the compression from $d$ to $a$. Representing by $\mathfrak{Q}_{1}$ the entire amount of heat energy taken from the hot source $A$ and equal to $A \times$ area abfe, and similarly representing by $\mathfrak{\Omega}_{2}$ the heat energy rejected to the cold source $B$, of amount $A \times$ area $c d h g$, we have, since the working substance returns to its original state, the net amount of heat supplied equal to the net amount of external work performed. Then $\mathfrak{D}_{1}-\mathfrak{Q}_{2}=A W$.

The efficiency of the engine is to be written as the ratio of the output $A W$ or $\mathfrak{Q}_{1}-\mathfrak{Q}_{2}$, to the input, which is $\mathfrak{Q}_{1}$. If $\eta$ represent the efficiency, we have then

$$
\eta=\frac{A W}{\mathfrak{\beth}_{1}}=\frac{\mathfrak{Q}_{1}-\mathfrak{Q}_{2}}{\mathfrak{\Omega}_{1}}
$$

25. Reversible Cycle. The Carnot cycle is reversible, inasmuch as it is possible to perform these operations in the reverse order, and to return the working substance to its initial state. Thus starting with the gas in condition represented by $a$, allow it to expand adiabatically to the state represented by $d$ in the pressure-volume plot. Then placing it upon the cold source $B$, allow the gas to expand isothermally from $d$ to $c$, while receiving an amount of heat $\mathfrak{Q}_{2}$. Then compress adiabatically from $c$ to $b$, where the temperature is $\theta_{1}$. And finally placing the cylinder upon the hot source $A$, compress isothermally from $b$ to $a$ and reject to the source an amount of heat $\mathfrak{\Omega}_{1}$. An amount of external work $W$, equal in value to $J\left(\mathfrak{Q}_{1}-\mathfrak{Q}_{2}\right)$, will have been done upon the gas, and an amount of heat $\mathfrak{Q}_{2}$ absorbed from the cold source and rejected to the hot source. This transfer of heat from a cold source to a warmer source has been at the expense of work performed by some external agency, and does not therefore constitute a violation of the Second Law:

26. Carnot's Theorem. Upon the assumption of the Second Law a theorem advanced by Carnot may be proved. This theorem states that all reversible heat engines have the same efficiency when absorb- 
ing and rejecting heat at the same limiting temperatures. To prove this statement imagine two heat engines, that is, two working sul)stances, operating in the fashion described in Section 2t. Let one of these, denoted by $A$, have a greater effieiency than the other engine, which will be represented by $B$. Imagine the mechanical details so arranged that when working with the same hot source and the same cold source the work done by $A$ shall equal that done by $B$. Then let $B$ pass through its eycle in the reversed direction, in a manner similar to that described in Section 25, and let the mechanical energy necessary to drive it be supplied by $A$. The work done by $A$ will then just equal that required to drive $B$, reversed. Let $A$ absorb from the hot source an amount of heat $\Sigma_{1}$, and reject to the cold source an amount $\mathfrak{Q}_{2}$. Similarly, let $B$ absorb from the cold source an amount $\mathfrak{\Omega}_{2}^{\prime}$, and reject to the hot source an amount $\mathfrak{Z}_{1}{ }^{\prime}$. Then if the heat taken from a source be indicated by a minus sign and that rejected to a source by a plus sign, the net result of the two operations upon the heat sources is shown in the following tabular arrangement:

Hot Source

Engine $A$

Engine $B$

Total heat

$$
\begin{array}{r}
-\mathfrak{\Xi}_{1} \\
+\mathfrak{\Xi}_{1}^{\prime} \\
\mathfrak{V}_{1}^{\prime}-\mathfrak{\Xi}_{1}
\end{array}
$$

Cold Source

$$
\begin{array}{r}
+\Omega_{2} \\
-\Omega_{2}^{\prime} \\
\Omega_{2}-\Omega_{2}^{\prime}
\end{array}
$$

Now since the work done by $A$ is equal to that which $B$ would do if passing through its cycle in the positive direction, it follows that

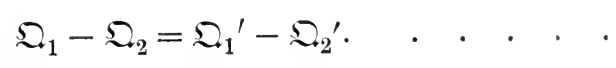

Also, since by assumption the efficiency of $A$ is greater than that of $B$,

$$
\frac{\Omega_{1}-\Omega_{2}}{\mathfrak{Q}_{1}}>\frac{\mathfrak{\Omega}_{1}^{\prime}-\mathfrak{\Omega}_{2}^{\prime}}{\mathfrak{\Omega}_{1}^{\prime}}
$$

Combining equations (i) and (ii) gives

$$
\begin{aligned}
& \frac{1}{\Omega_{1}}>\frac{1}{\Xi_{1}^{\prime}}, \\
& \mathfrak{\Omega}_{1}^{\prime}>\Omega_{1} .
\end{aligned}
$$

and hence

Further, by comparing equations (i) and (iii) it is evident that

$$
\mathfrak{\Xi}_{2}^{\prime}>\mathfrak{\Xi}_{2} \text {. }
$$

In the tabular arrangement above it appears therefore that an amount of heat is taken from the cold source and added to the hot 
source. The net result is that a self-acting machine (or combination of machines) performing a cycle is transferring heat from a cold body to a hot body without the performance of work by some external agency. This is impossible, according to the Second Law. Therefore $\mathfrak{l}_{1}^{\prime}$ cannot be greater than $\mathfrak{l}_{1}$ and also $\mathfrak{l}_{2}^{\prime}$ cannot be greater than $\mathfrak{Q}_{2}$.

Hence the efficiency of engine $A$ cannot be greater than that of engine $B$. A similar process of reasoning will show that the efficiency of $B$ cannot be greater than that of $A$. Hence the efficiencies of any two (or of all) reversible heat engines absorbing heat from the same hot source and rejecting heat to the same cold source must be the same.

27. Discussion of Reversible Processes. Planck says of reversible processes: " "A process which can in no way be completely reversed is termed irreversible; all other processes are reversible. That a process may be irreversible it is not sufficient that it cannot be directly reversed. This is the case with many mechanical processes which are not reversible. The full requirement is that it be impossible, even with the assistance of all agents in nature, to restore everywhere the exact initial state when the process has once taken place. The generation of heat by friction, the expansion of a gas without the performance of external work, the absorption of external heat, and the conduction of heat, and so on, are irreversible processes. Since there exists in nature no process entirely free from friction or heat conduction, all processes which actually take place in nature are in reality irreversible; reversible processes form only an ideal limiting case. They are, however, of considerable importance for theoretical demonstration and for application to states of equilibrium."

28. Discussion of the Second Law. In a summary of the foundations of thermodynamics Bryan says :2 "Passage of heat from one body to another is usually irreversible and therefore accompanied by a loss of available energy. If we define $A$ to be hotter or colder than $B$ according as available energy is lost or gained by the transference of heat from $A$ to $B$, it follows that heat can, and in general will, pass from hotter to colder bodies, but the reverse change can only be effected by combining it with a compensating transformation. Carnot's cycle reversed is a compensated reversible transformation by which heat can be continuously taken from a colder and given to a hotter body, or vice versa, without loss of availability.

1 Planck, “Thermodynamics," translated by Ogg, p. 85.

2 Bryan, “'Thermodynamies," Teubner, 1907. 
In this case the compensating transformation takes the form of work absorbed or produced."

The conclusion of Carnot's Theorem given in Section 26 is often stated as the Second Law. That theorem, however, holds only for reversible cyclic processes and is not as broad a statement of the Second Law as that quoted in Section 23, which is due chiefly to Clausius.' An excellent statement of the essence of the law is quoted from Drude ${ }^{1}$ as follows: "Mechanical work can'never be continually obtained at the expense of heat if only one source of heat at uniform temperature is at disposal."

From this statement there may easily be derived an alternative proof of Carnot's Theorem. Thus consider the engines $A$ and $B$ of Section 26, in which engine $A$ is assumed to be of greater efficiency than engine $B$, to be so arranged mechanically that they would each take from the same hot source the same amount of heat, but because of greater efficiency $A$ would convert more into work and reject less to the cold source than would $B$. Now imagine engine $A$ to drive engine $B$, reversed, and let $B$-give up to the hot source just as much heat as $A$ absorbs from it. Then the hot source may be omitted from consideration and $B$ supply $A$ directly. Because $A$. has a higher efficiency it will reject less heat to the cold source than will $B$ with its lower efficiency abstract from it. Further, $A$ will do more work than will $B$, and hence there will be a net amount of work available for external purposes. But this would be in violation of the Second Law, for this external work would be done as a result of a net amount of heat absorbed from the cold source ; that is, from the single source of heat at disposal. Hence there can be no external work. Hence, further, the efficiencies must be the same.

In this expression of the substance of the Second Law by Drude the emphasis may for the moment be placed upon the word " continually," which is essentially equivalent to the term "in a cyclic process." As an illustration of the fact that the Second Law holds only for cyclic processes, consider the following example. Imagine two non-conducting cylinders, one containing air at a high pressure and a low temperature and the other air at a low pressure and a high temperature. If they are fitted with connecting pistons so that the higher pressure air may expand and compress that in the other cyl-

1 Drude, "Theory of Optics," translated by Mann and Millikan, pp. 493-494. This statement is equivalent to that of Lord Kelvin ; namely, "It is impossible, by means of inanimate material agency, to derive mechanical effect from any portion of matter by cooling it below the temperature of the coldest of surrounding objects." 
inder, then, for this adiabatic change the temperature in the high pressure cylinder will fall, while that in the low pressure cylinder will rise. During the process, then, heat has been abstracted from the low temperature side and given to the high temperature side. This does not, however, constitute a violation of the Second Law, since the change is not cyclic. ${ }^{1}$

Imagine that the low pressure cylinder is in connection with the atmosphere as in a vacuum pump. The high pressure side then does external work in pushing out the atmosphere contained in the pump, and in so doing the working substance in the high pressure cylinder is cooled still farther below the temperature of the surrounding atmosphere. Heat may then be absorbed from the atmosphere and the original temperature thus regained. But still the working substance is not returned to its original state, and to return it would require as much work as was originally obtained from its expansion. The example cited is then in accordance with the Second Law.

In general, it may be said for the production of mechanical work two sources of heat at different temperatures are required, and the production of work requires the transfer of heat in such a direction as to tend to result in a final equality of temperature of the sources, in which condition all the available energy of the system will have been used up.

29. Thermodynamic Scale of Temperature. For a reversible cycle it has been seen that the efficiency of all heat engines is the same provided only that the temperature limits are the same. Lord Kelvin therefore proposed a thermodynamic scale of temperatures which is independent of the substance used.

Draw any two adiabatic lines for a substance and also two isothermal lines (for reasons evident later the isothermals will be those for temperatures of $0^{\circ}$ and $100^{\circ} \mathrm{C}$.). Let the area bounded by the two isothermals and the two adiabatics be divided into 100 equal parts by drawing other isothermals. Calling the area between any two adjacent isothermals $a$, divide the space below the zero degree Centigrade isothermal and between the two adiabatics into areas equal to $a$ by drawing other isothermals. In Figure 6 this has been represented by drawing the isothermals dividing the space between the $0^{\circ}$ and the $100^{\circ}$ isothermal into four parts. and extending this division below the $0^{\circ}$ isothermal as far as is possible in a finite

\footnotetext{
${ }^{1}$ In part this illustration is derived from Edser, "Heat for Advanced Students," p. 340.
} 
diagram. Let the lowest isothermal corresponding to the condition of the substance when there is no intrinsic molecular energy be

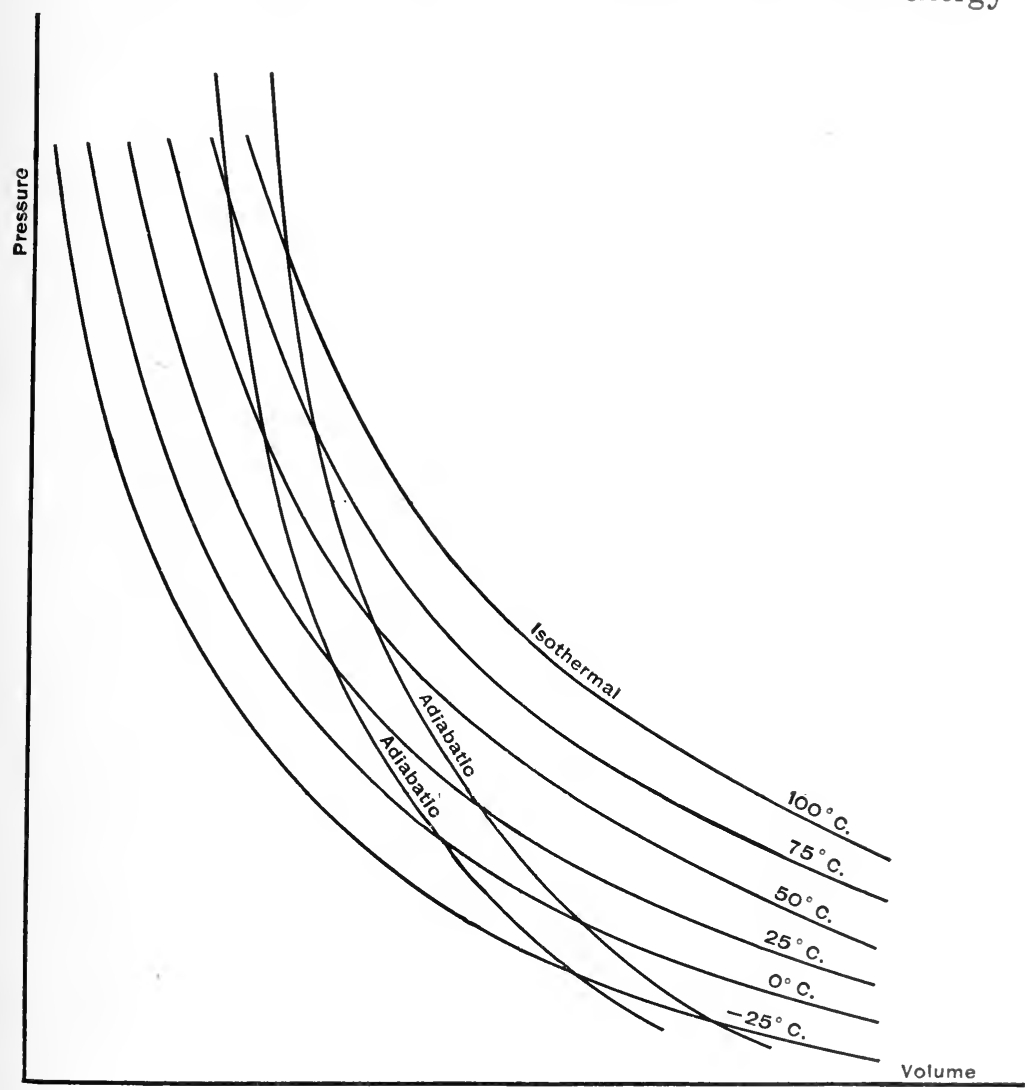

FIG. 6

called the absolute zero of temperature as in Section 15. Number the isothermals from this absolute zero, and let the number of any isothermal be represented by $T$.

Consider now a Carnot cycle composed of these two adiabatics and any two isothermals as $T_{1}^{\prime}$ and $T_{2}^{\prime}$, where $T_{1}^{\prime}$ is higher than $T_{2}^{\prime \prime}$. The efficiency of this cycle is $\frac{\mathfrak{Q}_{1}^{\prime}-\mathfrak{\Omega}_{2}^{\prime}}{\mathfrak{\Omega}_{1}^{\prime}}$, where $\mathfrak{\Omega}_{1}^{\prime}$ and $\mathfrak{\Omega}_{2}^{\prime}$ are measured as in Section 20 by the areas under the isothermal lines. The numerator of this expression is, however, equal to the area included by the two isothermals and the two adiabatics. If now a particular value of zero be given to $T_{2}^{\prime \prime}$, and if $T_{1}^{\prime}$ be put equal to $T$, then the expression for the efficiency of the Carnot cycle between 
these limiting isothermals becomes $\frac{\mathfrak{Q}-0}{\mathfrak{Q}}=\frac{T a}{\mathfrak{Q}}=1$. That is, $\mathfrak{Q}=T a$. This means merely that in traversing any isothermal between the two limiting adiabatics, the input is $T \times a$ in work units. Hence the efficiency of a Carnot cycle between two isothermals $T_{1}$ and $T_{2}$ is given by the expression

$$
\eta=\frac{\Omega_{1}-\Omega_{2}}{\Omega_{1}}=\frac{T_{1} a-T_{2} a}{T_{1} a}=\frac{T_{1}-T_{2}}{T_{1}^{\prime}} . \quad . \quad . \quad .
$$

If the numbers which have been assigned to the isothermals be considered to be temperatures, it is evident that we have a method for measuring temperature which is independent of the substance chosen. The zero of this thermodynamic scale coincides with the perfect gas thermometer zero, and the size of a degree is approximately the degree Centigrade as measured on a hydrogen thermometer. If now it can be shown that the efficiency of a Carnot cycle with a perfect gas for a working substance is $\frac{\theta_{1}-\theta_{2}}{\theta_{1}}$, where $\theta_{1}$ and $\theta_{2}$ are the absolnte temperatures on a perfect gas thermometric scale, it will be evident that the thermodynamic scale of Lord Kelvin is identical with the perfect gas scale.

30. Carnot Cycle for a Perfect Gas. It has already been shown

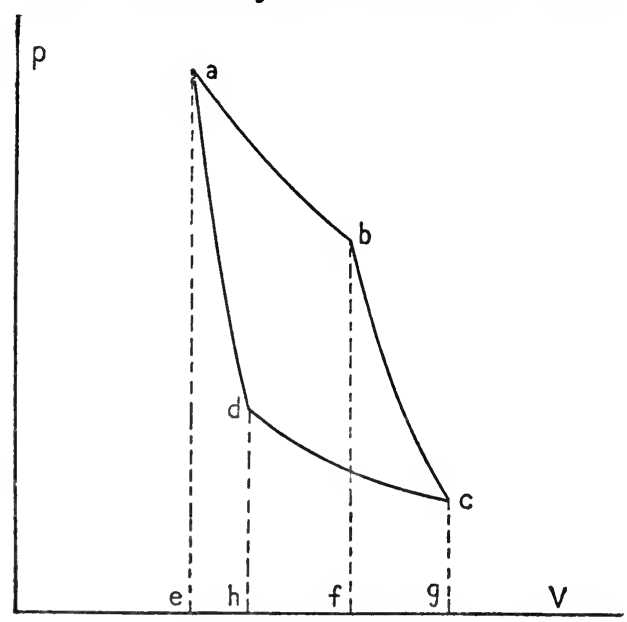

FIG. 7 that the work done by a perfect gas during the adiabatic expansion from $b$ to $c$ of the Carnot cycle shown in Figure 5 (reproduced here as Figure 7) is equal to that done upon the gas during the adiabatic compression from $d$ to $a$. It remains only to find the values of $\mathfrak{\Omega}_{1}$ and $\mathfrak{\Omega}_{2}$ in order to arrive at an expression for the efficiency of the Carnot cycle for a perfect gas. If the efficiency can be expressed entirely in terms of the absolute temperatures, then the comparison suggested in the preceding section can be made. According to equation (18) the area under the isothermal $a$ to $b$, which is equal to $J_{\mathfrak{Z}_{1}}$, is expressible as $m R \theta_{1} \log _{e}\left(V_{b} / V_{a}\right)$. And 
similarly, the heat rejected in passing along the isothermal from $c$ to $d$ is $\mathfrak{\Omega}_{2}=A m R \theta_{2} \log _{e}\left(V_{c} / V_{d}\right)$. The efficiency is therefore

$$
\begin{aligned}
& \eta=\frac{\mathfrak{Q}_{1}-\mathfrak{Q}_{2}}{\mathfrak{Q}_{1}}=\frac{A m R \theta_{1} \log _{e} \frac{V_{b}}{V_{a}}-A m R \theta_{2} \log _{e} \frac{V_{c}^{r}}{V_{a}^{r}}}{A m R \theta_{1} \log _{e} \frac{V_{b}}{V_{a}}} \\
& =\frac{\theta_{1} \log _{e} \frac{V_{b}}{V_{a}}-\theta_{2} \log _{e} \frac{V_{c}}{V_{d}}}{\theta_{1} \log _{e} \frac{V_{b}}{V_{a}}} .
\end{aligned}
$$

During the isothermal changes

$$
p_{a} V_{a}=p_{b} V_{b}=m R \theta_{1} \text {, and also } p_{c} V_{c}=p_{d} V_{d}=m R \theta_{2} .
$$

Hence

$$
\frac{p_{d} V_{d}}{p_{a} V_{a}}=\frac{\theta_{2}}{\theta_{1}} \text { and } \frac{p_{c} V_{c}}{p_{b} V_{b}}=\frac{\theta_{2}}{\theta_{1}}
$$

Similarly, for the adiabatic changes $p_{b} V_{b}{ }^{\kappa}=p_{c} V_{c}{ }^{\kappa}$ and $p_{d} V_{d^{\kappa}}{ }=p_{a} V_{a}{ }^{\kappa}$. Substituting from these adiabatic equations gives

$$
\begin{gathered}
\frac{\theta_{2}}{\theta_{1}}=\left(\frac{V_{d}}{V_{a}}\right)^{\kappa-1} \text { and } \frac{\theta_{2}}{\theta_{1}}=\left(\frac{V_{c}}{V_{b}}\right)^{\kappa-1}, \\
\frac{V_{d}}{V_{a}}=\frac{V_{c}}{V_{b}} \text { or } \frac{V_{b}}{V_{a}}=\frac{V_{c}}{V_{d}} .
\end{gathered}
$$

therefore

Substitution of this ratio in the expression for the efficiency gives

$$
\eta=\frac{\theta_{1}-\theta_{2}}{\theta_{1}}
$$

A comparison of equations (24) and (25) shows that a perfect gas scale is identical with the absolute thermodynamic scale.

31. Entropy, In equation (23), for the efficiency of a Carnot perfect gas eycle, if the two isothermals are taken at an infinitesimal difference of temperature apart, so that $\theta_{1}-\theta_{2}=d \theta$, then the corresponding heat $\mathfrak{Q}_{1}-\mathfrak{Q}_{2}$, converted into work, may be represented by the infinitesimal $d \mathfrak{Q}$, and by equation (25) the expression for the efficiency becomes

$$
\frac{d \mathfrak{Q}}{\mathfrak{Q}}=\frac{d \theta}{\theta} .
$$


The solution of this differential equation gives

$$
\begin{array}{r}
\log _{e} \frac{\mathfrak{\Omega}_{1}}{\mathfrak{\Omega}_{2}}=\log _{e} \frac{\theta_{1}}{\theta_{2}}, \text { or } \frac{\mathfrak{\Omega}_{1}}{\mathfrak{\Omega}_{2}}=\frac{\theta_{1}}{\theta_{2}}, \\
\frac{\mathfrak{I}_{1}}{\theta_{1}}=\frac{\mathfrak{\Omega}_{2}}{\theta_{2}} . . . . . .
\end{array}
$$

and hence

This means that for a reversible process the ratio of the heat absorbed (or rejected) to the absolute temperature of the isothermal traversed in passing between two adiabatics is independent of the isothermal traversed. The quantity which remains constant under these conditions is the difference in entropy of the substance in the states represented by the two adiabatics. An adiabatic line is then for a reversible process by definition a line of constant entropy, or an isentropic.

The entropy of any state will be represented by the usual symbol $\phi$. The expression $\frac{\varrho}{\theta}$ is then an expression for the difference in entropy, to be denoted by $\phi_{2}-\phi_{1}$, between the entropy $\phi_{1}$ of the first adiabatic and $\phi_{2}$, the entropy corresponding to the second adiabatic. This change in entropy between these two isentropics is independent of the path followed, provided only the path is a reversible one, as it was in the case of the isothermal of the Carnot cycle from which this concept was developed.

In general, if a small amount of heat $d \mathfrak{Q}$ be added to a body at a temperature $\theta$, which is sensibly constant during that addition, then the entropy of the body has been increased by an amount $d \phi$, where

$$
d \phi=\frac{d \Omega}{\theta} .
$$

If the change in entropy is produced by a large number of such additions, by processes which are completely reversible, the total change in entropy thereby produced is

$$
\phi_{2}-\phi_{1}=\int_{1}^{2} d \phi=\int_{1}^{2} \frac{7 \Omega}{\theta} . . . . .
$$

For the special case where the heat is added along an isothermal this reduces to

$$
\phi_{2}-\phi_{1}=\frac{\mathfrak{Z}}{\theta}
$$


Further, it is to be noticed that for the Carnot cycle

$$
\frac{\mathfrak{\vartheta}_{1}}{\theta_{1}}-\frac{\mathfrak{\vartheta}_{2}}{\theta_{2}}=0
$$

'That is, for a reversible cycle

$$
\left(\int\right) \frac{d \mathfrak{Q}}{\theta}=0, \quad \cdot \quad \cdot \quad \cdot \quad \cdot
$$

where the symbol $\left(\int\right.$ ) indicates the fact that the integration is to be performed about a complete cycle. This equation is sometimes stated as a mathematical form of the Second Law, but it is preferable to consider it a deduction from it.

32. Measurement of Entropy. It is not possible to determine the total entropy of a body. That is, the expression $\int \frac{d \Omega}{\theta}$ when used to find the entropy of a body in a given state will always contain a constant of integration. The integral does, however, arlmit of the determination of the difference in entropy between two given states. Hence it is that entropy is always expressed as so much more or less than the entropy of some chosen state of the substance under consideration. This is satisfactory for all practical uses of the concept of entropy. Having arbitrarily selected some adiabatic as a reference state to which all other states of the substance are to be compared, it is possible to conceive of the entire pressure-volume plot, for a given mass of the substance, as filled with adiabatic or isentropic lines which represent states of the substance differing by units of entropy, just as the states represented by the isothermal lines differ by units of temperature.

Quoting from Preston, " "The entropy of a body being arbitrarily taken as zero in some standard condition $A$ defined by some standard temperature and pressure (or volume), the entropy in any other state $B$ is the value of the integral $\int \frac{d \mathfrak{Q}}{\theta}$ taken along any reversible path by which the body may be brought to state $B$ from standard state $A$. The path may obviously be an are $A C$ of an isothermal passing through the point $A$, defining the standard state, together with the arc $B C$ of the adiabatic line passing through the point $B . "$

33. Temperature-Entropy Plot. It is evident from equation $(27)$ that, provided a change is taking place along a reversible path, the

1 Preston, “Heat,” p. 628. 
heat added (or rejected) may be expressed as the product of a temperature term and a difference in entropy term, thus

$$
\int_{1}^{2} d \cong=\int_{1}^{2} \theta d \phi . \quad . \quad \cdot \quad \cdot \quad \cdot
$$

For this reason and because of the practical interest in steam engineering of following the changes in entropy of steam it is most

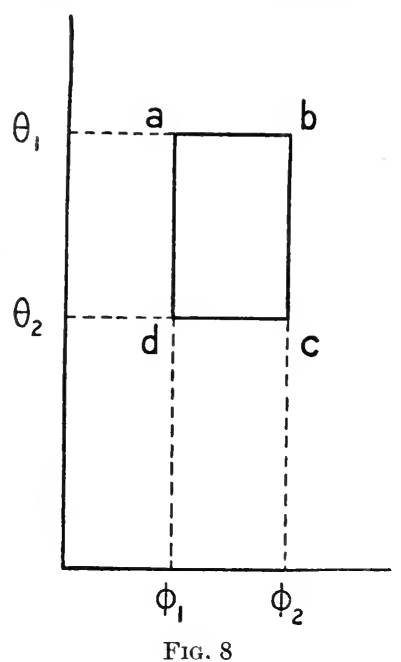
convenient to plot values of entropy on a temperature-entropy plot.

In the temperature-entropy, or " $\theta-\phi$ plot," the ordinates are temperatures and the abscissas are entropies measured from some standard state as described above. The $\theta-\phi$ diagram of the Carnot cycle will now be plotted as an illustration. During the isothermal expansion from $a$ to $b$ of Figure 7 , the entropy increases by an amount by $\phi_{2}-\phi_{1}$, and the change is represented as $a b$ in the plot of Figure 8 . The reversible adiabatic expansion from $b$ to $c$ is isentropic by definition and is represented on the $\theta-\phi$ plot by the line $b c$. The isothermal or constant temperature change $c d$ and the reversible adiabatic compression $d a$ complete the cycle.

It is to be noticed that for this case, since the isothermal is a reversible process, the heat absorbed at temperature $\theta_{1}$ is represented by the area under the line $a b$. Similarly, the heat rejected during the isothermal compression from $c$ to $d$ is given by the area under the line $c d$. The net heat converted into work in this cycle is then $\mathfrak{Q}_{1}-\mathfrak{Q}_{2}=\theta_{1}\left(\phi_{2}-\phi_{1}\right)-\theta_{2}\left(\phi_{2}-\phi_{1}\right)=\left(\theta_{1}-\theta_{2}\right)\left(\phi_{2}-\phi_{1}\right)$; that is, it is represented by the area enclosed by the $\theta-\phi$ plot of the cycle.

It is important to note carefully that it is only when the transformation takes place in a reversible manner that the heat absorbed (or rejected) is represented by the area underneath the curve on the $\theta$ - $\phi$ diagram representing the transformation. In all other cases the increase in entropy will be disproportionate to the increase in heat contents as in the case of the porous plug experiment to be described in Section 35. Although this restriction limits this use of a $\theta-\phi$ plot to the ideal cases, such a plot is still of great interest and value in many practical problems. 
34. Discussion of Carnot Cycle from $\theta-\phi$ Plot. If any other reversible cycle be plotted upon the $\theta-\phi$ diagram as in Figure 9, it is evident that the work output, which would be proportional to the area enclosed by the plot, is less than for the Carnot cycle, although the same range of temperatures is used. It follows then that no reversible heat engine can have an efficiency greater than that of the Carnot engine. And of course all heat engines with irreversible transformations are less efficient for the same limiting temperatures than the reversible engines. An example of a reversible cycle equal in efficiency to the Carnot cycle is given in Problem 11, $\theta_{2}$ on page 51 .

It is evident from Figures 8 and 9 that the high efficiency of the Carnot cycle is obtained as the result of two conditions, namely, that all the heat absorbed is taken in at the highest temperature, and all the heat rejected is at the lowest temperature. In so far as any heat engine conforms to

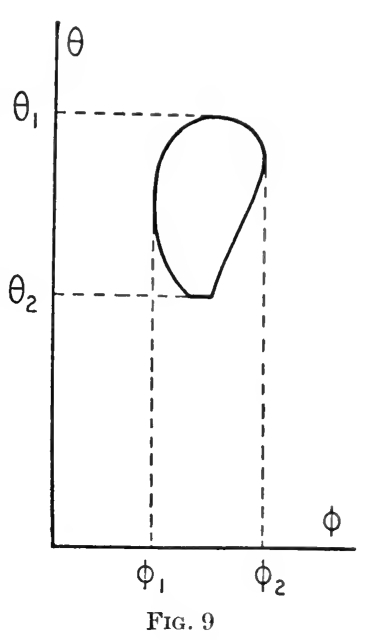
these requirements (neglecting the fact that its other transformations will not in general be isentropic) will its efficiency approach the limiting and maximum efficiency of a Carnot engine working between the same temperatures.

35. Entropy Changes in Irreversible Processes. Returning again to the concept of entropy, it may be said that entropy is that property of a body which remains constant so long as the body is undergoing adiabatic changes that are reversible. If the change is not reversible, then the entropy in the final state is greater than it would be for a reversible transformation. This is indicated by the classical "porous plug experiment" of Kelvin and Joule. In this experiment, which offers a more accurate method than does that described in Section 11 for testing Joule's Law, it was found that when air expanded from a higher pressure to a lower pressure without doing external work there was a distinct lowering of the temperature, due to the partial conversion of the kinetic energy of the molecules into potential energy of molecular separation. The experiment was performed by allowing air to expand from a high pressure through a plug of cotton wool to atmospheric pressure. The plug was surrounded with a non-conductor and the expansion was adiabatic. 
For the purposes of the present discussion the important point of the experiment is as follows. A mass of the gas reaching an opening in the plug, upon the other side of which the pressure is lower, expands adiabatically (a reversible and hence isentropic change), converting a portion of its intrinsic energy into mechanical kinetic energy of the entire mass. This mechanical kinetic energy is immediately converted into heat by friction, viscosity, and the like. The quantity of heat corresponding to this friction work is now returned to the air and causes an increase in entropy, exactly as if it had been added from some outside source. As a result of these two infinitesimal changes, the first isentropic and the second of increasing entropy, the final entropy of the air is obviously increased. The same conclusions as to an increase in entropy may be made for the original experiment of Joule, but it is easier to conceive of the steps in this case. This expansion, although adiabatic,

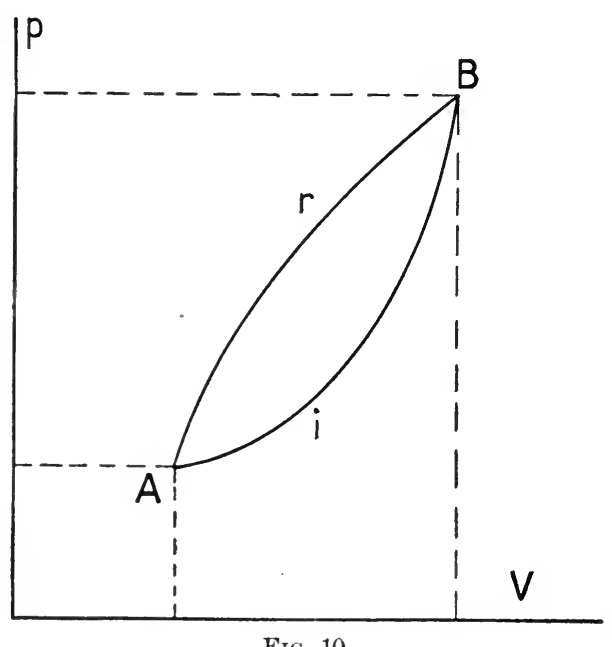

FIG. 10 is obviously irreversible and the entropy is increased. For further discussion it will be assumed to have been shown that all irreversible transformations lead to a final state of greater entropy than would a corresponding reversible transformation.

Consider a change of state for a given substance from $A$ to $B$ by a reversible path as represented on the pressurevolume plot of Figure 10 by the line $A r B$. This change might be represented on the temperature-entropy plot of Figure 11 by the line $A r B$. If now the transformation from $A$ to $B$ is along some irreversible path as $A i B$ in Figure 10, it may be represented on the $\phi-\theta$ plot by some line $A i B^{\prime}$, since the final entropy is greater than for the reversible change. The integral $\int_{A}^{B} \frac{d \mathfrak{Q}}{\theta}$ gives the change in entropy along the reversible path, but is less than the true change along the irreversible path. Thus

$$
\phi_{B^{\prime}}-\phi_{A}>\int_{A}^{B} \frac{d \mathfrak{Q}}{\theta} \text {. . . . . . . }
$$


Similarly, if the transformation take place from $B$ to $A$, the entroly along the reversible path is given by the integral $\int_{B}^{-1} \frac{7 \Omega}{\theta}$. Wut the entropy in the final state for the irreversible path is greater than that for the reversible path. That is,

$$
\phi_{A^{\prime}}-\phi_{B}>\int_{B}^{A} \frac{1}{\theta} \text {. }
$$

For the irreversible change, represented on the $p$-v plot by ArBiA the total change in entropy is not zero as it would be for an entirely reversible process, but is obviously greater than zero (or at the most equal to it). For, from $A$ to $B$ by a reversible process

$\phi_{B}-\phi_{A}=\int_{A}^{B} \frac{d \mathfrak{Q}}{\theta},$.

and from $B$ to $A$ by an irreversible process

$\phi_{A^{\prime}}-\phi_{B}>\int_{B}^{A} \frac{d \mathfrak{Q}}{\theta}$

Then by the path $A r B i A$

$$
\phi_{A^{\prime}}-\phi_{A}>\int \frac{d \mathfrak{Q}}{\theta}=0 \text {. }
$$

That is, for the irreversible process just considered, the

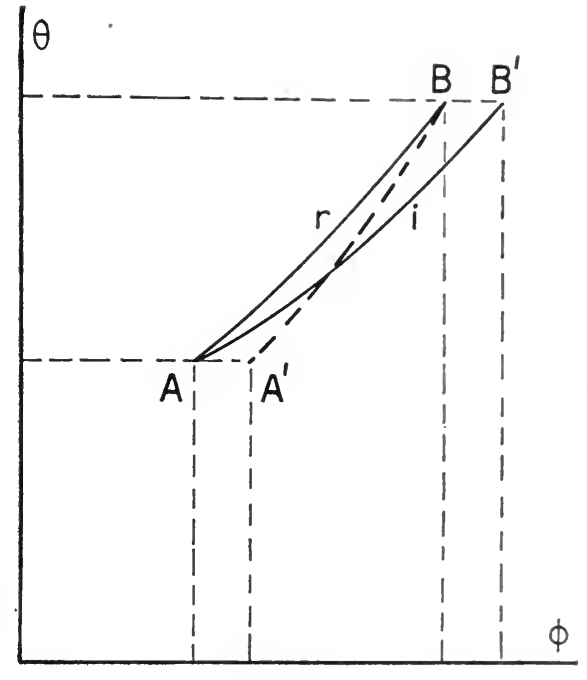

FIG. 11 expression $\int \frac{d פ}{\theta}$ taken for the entire change is less than the actual increase of entropy. But the physical state represented by $\phi_{A^{\prime}}$ is not the same as the initial physical state of entropy $\phi_{A}$. In order, then, that the process shall be a true cycle, it is necessary to subtract an amount of entropy of value in this case $\phi_{A^{\prime}}-\phi_{A^{\prime}}$. This fact, usually stated in the form given below, is due to Clausius.

$$
\left(\int\right) \frac{d g}{\theta}=0 . \cdots \cdot \cdot \cdot \cdot \cdot
$$




\section{CHAPTER II}

\section{GASES}

36. Graphical Representation of State for a Perfect Gas. It is a simple fact of solid analytical geometry that a function of three variables in general represents a surface. The general equation for a given mass $m$ of a perfect gas, namely $p V=m R \theta$, is evidently a function of three variables and may be taken as representing a surface plotted with reference to three rectangular axes, $p, V$, and $\theta$, corresponding to $x, y$, and $z$. This surface is known as the "characteristic surface" of the gas. Since the position of any point on this surface represents a corresponding state or condition of the gas, such a point is usually termed a "state point" of the gas. Now, whatever changes the given mass of gas may undergo, its successive states must be represented by state points on this characteristic surface, corresponding to the successive values of $p, V$, and $\theta$ which the gas assumes during its transformation. The continuous curve passing through these points is a curve in space and has an equation corresponding to the type of transformation which the gas has undergone.

The equation in rectangular coördinates of a curve in space is usually expressed by two equations, each containing two variables only. These two equations represent two cylinders, which are right projecting cylinders of the curve upon the two coördinate planes corresponding to the two sets of variables in which the equations are expressed. The intersection of these two cylinders determines completely the curve in space. In the case of a curve in space representing a gas transformation, since the curve must lie upon a known surface, one equation in addition to the equation for this characteristic surface is sufficient. This equation might be expressed in terms of $p$ and $V, p$ and $\theta$, or $V$ and $\theta$.

The projection on the $p$ and $V$ plane is most serviceable for ordinary purposes, as is evident from the discussion of Sections 19 and 20. It is to be noticed that the expressions there given in $p$ and $V$ coördinates for various type transformations represent equations for a right projecting cylinder of the space curve, perpendicular to the 
pressure-volume plane, and also that the third coördinate has already been found from the characteristic equation by substitution of the values of $p$ and $V$.

In general, a characteristic surface of unit mass of any substance may be represented by the equation stating the relation of any three of the five following magnitudes: specific pressure, specific volume, absolute temperature, intrinsic energy, and entropy. This is true because in general if any two of these magnitudes are known for a given state of the substance, the values of the remaining three for this state may be expressed as functions of the first two. Two exceptions occur, one evidently in the ease of perfect gases (and for practical purposes in the case of all gases) inasmuch as the absolute temperature of a gas is directly proportional to its intrinsic energy. (The other exception is in the case of a vapor where the temperature and the pressure do not completely determine the other magnitudes.)

The expression of a transformation in terms of the absolute temperature and the entropy is frequently of value, and the temperatureentropy plot will be used throughout the remainder of this text. ${ }^{1}$ Determinations of entropy and intrinsic energy from direct measurements are of course impossible. The three magnitudes that may be directly measured, namely, the temperature, pressure, and volume, are all easily found. For this reason, as well as because of the relation between work and area on a $p$-v plot (see Section 19), the pressure-volume plot is used almost exelusively in practical work.

37. Equations for Polytropic Transformation of a Perfect Gas. The equation for the most general type of gas transformation has been given in Section 19 as

$$
p_{1} V_{1}^{n}=p_{2} V_{2}^{n}=\text { a constant. }
$$

For $n$ equal to unity this equation expresses the relations of an isothermal change, and for $n$ equal to $\kappa$ the relations of an adiabatic change.

Since it is frequently desirable to express some special form of such a polytropic transformation in terms of $p$ and $\theta$, or of $V$ and $\theta$, general equations for that purpose will now be derived. Combining equation (i) with the relation for a perfect gas, namely,

$$
\frac{p_{1} V_{1}}{\theta_{1}}=\frac{p_{2} V_{2}}{\theta_{2}}, \quad \cdot \quad \cdot \quad \cdot \quad \cdot \quad \cdot \quad \cdot .
$$

1 A pressure-entropy plot is sometimes used in superheated steam problems, as is discussed on page 96 . 
gives

or

$$
\theta_{1} V_{1}^{n-1}=\theta_{2} V_{2}^{n-1}, \quad \cdot \quad \cdot \quad \cdot \quad \cdot \quad \cdot \text { (iii) }
$$

Also from (i) and (ii)

$$
\frac{Y_{1}}{T_{2}}=\left(\frac{\theta_{2}}{\theta_{1}}\right)^{\frac{1}{n-1}}
$$

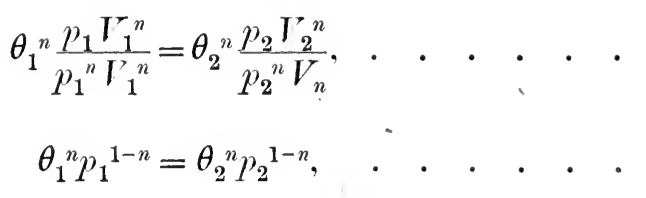

hence

or

$$
\frac{p_{1}}{p_{2}}=\left(\frac{\theta_{1}}{\theta_{2}}\right)^{\frac{n}{n-1}} .
$$

38. Fundamental Heat Equations of Perfect Gas. Since a gas transformation may be represented by the projection of the state curve upon the plane of $p$ and $V, p$ and $\theta$, or $V$ and $\theta$, it follows that the effect of adding to a given mass (as unit mass) a small amount of heat di may be expressed in terms of any two of these variables. It is to be remembered that this has already been done in terms of $p$ and $v$ in Section 21, where the result was expressed in equation (21), which is here reproduced.

$$
d \mathfrak{q}=c_{v} \frac{v}{R} d p+c_{p} \frac{p}{R} d v
$$

This equation will, however, be put in its more usual form by substituting from the equation for unit mass of a perfect gas, namely,

$$
p v=R \theta, \text {. . . . . . . . . . . }
$$

the value of $\frac{\theta}{v}$ for $\frac{p}{R}$ and also $\frac{\theta}{p}$ for $\frac{v}{R}$, giving equation (33) as below,

$$
d \mathfrak{q}=c_{p} \theta \frac{d v}{v}+c_{v} \theta \frac{d p}{p} . \quad . \quad . \quad . \quad .
$$

From equation (i) we have also

and

$$
d v=\frac{\partial v}{\partial \theta} d \theta+\frac{\partial v}{\partial p} d p=\frac{R}{p} d \theta-\frac{R \theta}{p^{2}} d p, \quad . \quad . \quad .
$$

$$
d p=\frac{\partial p}{\partial \theta} d \theta+\frac{\partial p}{\partial \eta} d v=\frac{R}{v} d \theta-\frac{R \theta}{v^{2}} d v . \quad . \quad .
$$


Substituting from (ii) in (33) gives equation (3t), which expresses in terms of the temperature, $\theta$, and pressure, $p$, the same effect as does (33). Thus

Hence

$$
d \mathfrak{q}=c_{p} \frac{\theta}{v}\left[\frac{R}{p} d \theta-\frac{R \theta}{p^{2}} d p\right]+c_{v} \frac{\theta}{p} d p=c_{p} \frac{R \theta}{p v} d \theta-\left(c_{p} \frac{R \theta^{2}}{p^{2} v}-c_{v} \frac{\theta}{p}\right) d p .
$$

$$
d \mathfrak{q}=c_{p} d \theta-\left(c_{p}-c_{v}\right) \theta \frac{d p}{p}
$$

Similarly, substituting from (iii) in (33) gives $d \mathfrak{q}$ in terms of $v$ and $\theta$. Thus

$$
d \mathfrak{q}=c_{r} d \theta+\left(c_{p}-c_{v}\right) \theta \frac{d v}{v}
$$

39. Entropy Change for Perfect Gas. From the three equations derived above there are easily obtained three expressions for the difference of entropy between two states of a gas provided the change produced may be eonsidered reversible. In case of a reversible transformation

$$
\phi_{2}-\phi_{1}=\int_{1}^{2} \frac{d \mathfrak{q}}{\theta} .
$$

If the coördinates of state 1 be represented by $p_{1}, v_{1}$, and $\theta_{1}$, and similarly the coördinates of state 2 by $p_{2}, v_{2}$, and $\theta_{2}$, then we may obtain from equation (33) the following expression for the difference in entropy:

or

$$
\phi_{2}-\phi_{1}=\int_{1}^{2}\left[c_{v} \frac{d p}{p}+c_{p} \frac{d v}{v}\right]=\int_{p_{1}}^{p_{2}} c_{v} \frac{d p}{p}+\int_{v_{1}}^{v_{2}} c_{p} \frac{d v}{v}
$$

$$
\phi_{2}-\phi_{1}=c_{v} \log _{e} \frac{p_{2}}{p_{1}}+c_{p} \log _{e} \frac{v_{2}}{v_{1}} . \quad . \quad . \quad \text {. }
$$

And similarly, equations (34) and (35) lead to equations (37) and (38), as follows :

$$
\begin{aligned}
& \phi_{2}-\phi_{1}=c_{v} \log _{e} \frac{\theta_{2}}{\theta_{1}}+\left(c_{p}-c_{v}\right) \log _{e} \frac{v_{2}}{v_{1}} \\
& \phi_{2}-\phi_{1}=c_{p} \log _{e} \frac{\theta_{2}}{\theta_{1}}-\left(c_{p}-c_{v}\right) \log _{e} \frac{p_{2}}{p_{1}}
\end{aligned}
$$

Any one of these three equations may be used to calculate the change in entropy. Thus if the change is isothermal, (37) and (38) both reduce to a simple form, since the logarithm of the term involving the temperature reduces to zero in this case. Similarly, 
for a constant volume change, (36) or (37) may most easily be used. It is important to notice that, since these equations all involve the specific heat and were derived for unit mass of the gas, the values of the entropy found by their use is in every case per unit mass of the substance, and to obtain the total entropy change the value $\phi_{2}-\phi_{1}$ must be multiplied by $m$. With this understanding it makes no difference whether the ratio of the volumes is $v_{1} / v_{2}$ or $V_{1} / V_{2}$, since these are equivalent ratios.

40. Relation of the Specific Heats of a Gas. It has already been stated in Section 18 that $c_{p} / c_{v}$ is a ratio, $\kappa$, that depends upon the

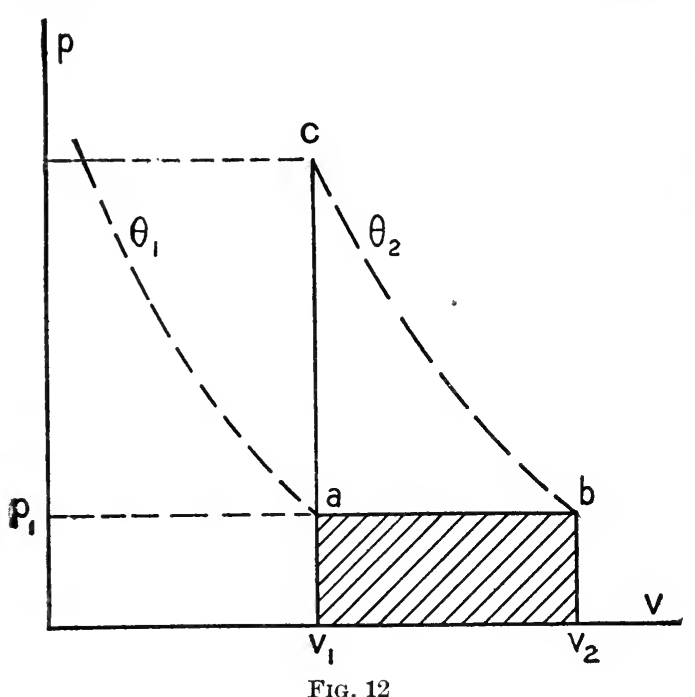
nature of the gas. It will now be shown that $c_{p}-c_{v}=A R$, where $R$ is the constant of the characteristic gas relation.

If unit mass of a perfect gas at a pressure, $p$, and a volume, $v$, is heated from $\theta_{1}$ to $\theta_{2}$ under the conditions of constant volume, the $p$ - $v$ plot of the change will be as shown in Figure 12 by the line $a c$. The heat supplied is $c_{v}\left(\theta_{2}-\theta_{1}\right)$. Since no external work is done.by the gas, this expression represents in heat units the increase in intrinsic energy of the gas. If, however, the change from $\theta_{1}$ to $\theta_{2}$ be made at constant pressure, as represented by the line $a b$ in the figure, then the heat added is $c_{p}\left(\theta_{2}-\theta_{1}\right)$. Since the final temperatures are the same, the increases in intrinsic energy are the same. During the heating at constant pressure an amount of external work represented by the shaded area of the figure has been done. The value in thermal units of this external work is $A p\left(v_{2}-v_{1}\right)$. It is evident, then, that

or

$$
c_{p}\left(\theta_{2}-\theta_{1}\right)-c_{v}\left(\theta_{2}-\theta_{1}\right)=A p\left(v_{2}-v_{1}\right), \quad . \quad \text {. }
$$

$$
c_{p}-c_{v}=\frac{A p\left(v_{2}-v_{1}\right)}{\theta_{2}-\theta_{1}}, \text {. . . . . . . }
$$

but

$$
p v_{1}=R \theta_{1} \text { and } p v_{2}=R \theta_{2} . \quad . \quad . \quad \text {. }
$$


From (iii) we have

$$
p\left(v_{2}-v_{1}\right)=R\left(\theta_{2}-\theta_{1}\right)
$$

Hence substituting from (iv) in (ii) gives

$$
c_{p}-c_{v}=A R \text {. }
$$

41. Changes in Intrinsic Energy of a Gas. For a perfect gas the intrinsic energy consists entirely, as has been said in Section 15, of molecular kinetic energy and is directly proportional to the absolute temperature. 'The value of the intrinsic energy of a gas at a given pressure and occupying a given volume may then be expressed in terms of this pressure and volume in accordance with the following reasoning. Imagine the gas to expand adiabatically from the state $a$, at which the value of the intrinsic energy is desired, until the pressure is zero. During this expansion all the intrinsic energy is converted into

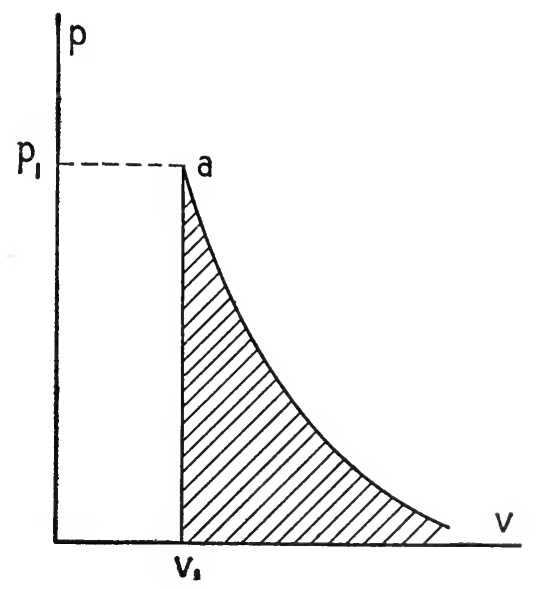

Fig. 13 external work. Such an expansion is represented diagrammatically in Figure 13. The energy may therefore be measured by the area under the adiabatic drawn through $a$ as in the figure. Denoting the energy in state $a$ as $E_{1}$, we have, according to equation (19) of Section 20,

$$
E_{1}=\frac{p_{1} V_{1}}{\kappa-1}\left[1-\left(\frac{V_{1}}{V_{2}}\right)^{\mathbf{\kappa}-1}\right]
$$

In this equation the volume $V_{2}$, corresponding to zero pressure, will be infinite and hence the expression for $E_{1}$ reduces to

$$
E_{1}=\frac{p_{1} V_{1}}{\kappa-1} \cdot \text {. . . . . . . . . }
$$

This may also be written as

$$
E_{1}=\frac{m R \theta_{1}}{\kappa-1}
$$

From this form it is evident that the value given in equation ( 40$)$ contains the mass implicitly, and hence the above expressions for intrinsic energy are for the total energy and not per unit mass of the gas. 
The difference in intrinsic energy between two states 1 and 2 of the same mass of gas is evidently

$$
E_{2}-E_{1}=\frac{p_{2} V_{2}-p_{1} V_{1}}{\kappa-1}
$$

In order that this energy shall be expressed in foot pounds it is necessary that $p$ be expressed in pounds per square foot and $V$ in cubic feet.

42. Imperfect Gases. As has been previously stated, for ordinary engineering purposes it is sufficient to apply the relations established in this chapter for perfect gases to any of the ordinary so-called "permanent gases" such as hydrogen, oxygen, nitrogen, marsh gas, and mixtures like air (and also, to some extent, to carbon dioxide and the like). When it is desired to analyze the action of any mixture, it may frequently be done by the use of Dalton's Law of partial pressures, which states that if $p_{1}$ and $p_{2}$ represent the pressures which two chemically inert gases would exert at the same temperature when separately occupying a volume $V$, then the resultant pressure $p$ for that temperature due to both these gases confined in this volume is the sum of $p_{1}$ and $p_{2}$.

Certain constants for the ordinary gases are to be found in Table 2 on page 130 .

43. Résumé of Equations for Cases.

The numbers following the equations are the original numbers assigned to them in the preceding chapters.

Temperature.

$\theta=459.5+t$ Fahrenheit ; $\theta=273+t$ Centigrade. . .

External Work.

$$
W=\int_{V_{1}}^{V_{2}} p d V \cdot \quad \cdot \cdot \cdot \cdot \cdot \cdot
$$

Characteristic Equation.

$$
p V=m R \theta \quad \quad \cdot \quad \cdot \quad \cdot \quad \cdot \quad \cdot
$$

Specific Heat.

$$
\begin{aligned}
& \text { - } c_{p}=\left(\frac{d \mathfrak{q}}{d t}\right)_{p} \text { and } c_{v}=\left(\frac{d \mathfrak{q}}{d t}\right)_{v} \text {. . . . . } \\
& \frac{c_{p}}{c_{v}}=\kappa . \quad \cdot \quad \cdot \quad \cdot \quad \text { (Section 18) } \\
& c_{p}-c_{v}=A R \text {. . . . . . . } \\
& \Omega=A W \ldots \quad \cdot \quad \cdot \quad . \quad \text {. . . . } \\
& d \Omega=\theta d \phi . \quad \text {. . . . . . . }
\end{aligned}
$$

General Heat Equations. 


$$
\begin{gathered}
d \mathfrak{l}=A(d S+d I+d W)=A(d E+d W) . \\
d \mathfrak{q}=c_{p} \theta \frac{d v}{v}+c_{v} \theta \frac{d p}{p} \cdot . . \\
d \mathfrak{q}=c_{v} d \theta+\left(c_{p}-c_{v}\right) \theta \frac{d v}{v} \cdot . \\
d q=c_{p} d \theta-\left(c_{p}-c_{v}\right) \theta \frac{d p}{p} . .
\end{gathered}
$$

Isothermal Transformation.

$$
\begin{gathered}
p_{1} V_{1}=p_{2} V_{2} \ldots \\
W=p_{1} V_{1} \log _{e} \frac{V_{2}}{V_{1}}=p_{1} V_{1} \log _{e} \frac{p_{1}}{p_{2}}=m R \theta_{1} \log _{e} \frac{V_{2}}{\Gamma_{1}} .
\end{gathered} .
$$

Adiabatic Transformation.

$$
\begin{aligned}
& p_{1} V_{1}{ }^{k}=p_{2} V_{2}{ }^{\kappa} \cdot \quad \cdot \quad \cdot \quad \cdot \quad \cdot \\
& \frac{p_{1}}{p_{2}}=\left(\frac{T_{2}}{V_{1}}\right)^{\kappa}=\left(\frac{\theta_{1}}{\theta_{2}}\right)^{\kappa}{ }^{\kappa} \\
& \frac{V_{1}}{V_{2}}=\left(\frac{p_{2}}{p_{1}}\right)^{\frac{1}{\kappa}}=\left(\frac{\theta_{2}}{\theta_{1}}\right)^{\frac{1}{\kappa-1}} \text {. } \\
& W=\frac{p_{1} T_{1}}{\kappa-1}\left[1-\left(\frac{T_{1}}{\Gamma_{2}}\right)^{\kappa-1}\right]=\frac{p_{1} \Gamma_{1}-p I_{2}}{\kappa-1} . .
\end{aligned}
$$

Intrinsic Energy.

$$
\begin{gathered}
E_{1}=\frac{p_{1} V_{1}}{\kappa-1} \cdot . \quad . \quad . \\
E_{2}-E_{1}=\frac{p_{2} T_{2}-p_{1} V_{1}}{\kappa-1} \cdot .
\end{gathered}
$$

Entropy.

$$
\begin{gathered}
d \phi=\frac{d \mathfrak{Q}}{\theta} \ldots \\
\phi_{2}-\phi_{1}=c_{v} \log _{e} \frac{p_{2}}{p_{1}}+c_{p} \log _{e} \frac{T_{2}}{\Gamma_{1}} \\
\phi_{2}-\phi_{1}=c_{v} \log _{e} \frac{\theta_{2}}{\theta_{1}}+\left(c_{p}-c_{v}\right) \log _{e} \frac{T_{2}}{I_{1}} \ldots \\
\phi_{2}-\phi_{1}=c_{p} \log _{e} \frac{\theta_{2}}{\theta_{1}}-\left(c_{p}-c_{v}\right) \log _{e} \frac{p_{2}}{p_{1}} \ldots
\end{gathered}
$$

Carnot Cycle.

$$
\eta=\frac{\mathfrak{Q}_{1}-\mathfrak{Q}_{2}}{\mathfrak{\Omega}_{1}}=\frac{\theta_{1}-\theta_{2}}{e_{1}} \quad . \quad .
$$




\section{PROBLEMS AND SOLUTIONS: GASES}

1. Use of the characteristic equation. A gas tank contains 1.5 pounds of oxygen at a temperature of $65^{\circ}$ Fahrenheit and a gauge pressure of 50.3 pounds per square inch. The atmospheric pressure is standard atmospheric pressure. Find how much more oxygen the tank can contain and not exceed its safe pressure limit of 250 pounds gauge, if the temperature is liable to rise to $100^{\circ}$.

Solution. A gauge always reads the difference between the pressure which it is to measure and the atmospheric pressure. Normal or standard atmospheric condition is a pressure of 14.7 pounds per square inch.

First, find the volume of the tank from eq. (13) and Table 2,

$$
\begin{gathered}
p=(50.3+14.7)(144), \quad \theta=459.5+65=524.5, \quad m=1.5, \quad R=52.56 . \\
\therefore V=\frac{(1.5)(52.56)(524.5)}{(65)(144)}=4.42 \text { cubic feet. }
\end{gathered}
$$

Then, find the weight of oxygen which the tank can contain under the conditions

$$
\begin{gathered}
p=(250+14.7)(144), \quad \theta=459.5+100=559.5, \\
m=\frac{(264.7)(144)(4.42)}{(52.56)(559.5)}=5.73 \text { pounds } .
\end{gathered}
$$

The added weight is then $5.73-1.5=4.23$ pounds.

2. Carnot cycle. A Carnot engine working between $350^{\circ}$ Fahrenheit and $75^{\circ}$ does 116,700 foot pounds of work per cycle. Find how much heat is supplied and rejected per cycle.

$$
\begin{aligned}
& \text { Solutior. } \quad \theta_{1}=459.5+350=809.5, \quad \theta_{2}=459.5+75=534.5 \text {, } \\
& \eta=\frac{\theta_{1}-\theta_{2}}{\theta_{1}}=\frac{809.5-534.5}{809.5}=0.34 \text {. } \\
& W=116.700 \text { foot pounds, } \quad A W=7^{\frac{1}{78}}(116,700)=150 \text { B.t.u. } \\
& \eta \mathfrak{Q}_{1}=A W . \quad \therefore \mathfrak{Q}_{1}=\frac{150}{0.34}=442 \text { B.t.u. } \\
& \mathfrak{Q}_{1}-\mathfrak{Q}_{2}=A W . \quad \therefore \mathfrak{Q}_{2}=442-150=292 \text { B. t. u. }
\end{aligned}
$$

3. Intrinsic energy. What is the energy of 2 pounds of air at 150 pounds per square inch pressure, absolute, if it occupies 6 cubic feet?

Solution. $\quad p=(150)(144), \quad V=6, \quad m=2, \quad \kappa=1.40$.

By eq. (40), $\quad E=\frac{p V}{k-1} . \quad \therefore E=\frac{(150)(144)(6)}{1.40-1}=324,000$ foot pounds.

Alternative solution. Find the temperature $\theta$, and assuming that the value of $c_{v}$ holds from the absolute zero to this temperature, calculate the number of thermal units to raise the temperature of 2 pounds of air from zero to $\theta$. Then reduce thermal units to mechanical energy units. 
By eq. (13),

$$
\begin{aligned}
\theta & =\frac{(150)(144)(6)}{(53.35)(2)}=1215, \\
E & =m c_{v} \theta=(2)(0.169)(1215)=411 \text { B. t. u. } \\
\therefore E & =(411)(778)=320,000 \text { foot pounds. } 1
\end{aligned}
$$

4. Constant pressure transformation. If 2 pounds of air are heated at constant pressure from $60^{\circ}$ Fahrenheit to $200^{\circ}$ find how much external work is done and how much the intrinsic energy is increased. How much heat is required?

Solution. $\quad c_{p}=0.2375, \quad c_{p}-c_{v}=A R, \quad R=53.35, \quad m=2, \quad t_{1}=60, \quad t_{2}=200$. The heat required at constant pressure is $c_{p} m\left(t_{2}-t_{1}\right)=(0.2375)(2)(200-60)=66.5$ B.t.u. External work done is $\left(c_{p}-c_{v}\right)(m)\left(\dot{t_{2}}-t_{1}\right)=A R(2)(200-60)=\frac{53.35}{778}(2)(140)=19.2$.

Increase in intrinsic energy is . . . . . . . . . $4 \overline{47.3 \mathrm{~B} . \mathrm{t} . \mathrm{u} .}$ Or, expressed in mechanical units, it is $(47.3)(778)=36,800 \mathrm{ft}$. $\mathrm{lb}$.

5. Exponent $n$. Given 2 pounds of air at a pressure of 14.7 pounds absolute which occupy a volume of 24.78 cubic feet. If the air expands until the pressure in $0.4 p_{1}$ and the volume is $V_{2}=2 V_{1}$, find the exponent $n$ in the equation $p V^{n}=p_{1} V_{1}^{n}$, which may be taken to represent the expansion.

Solution. $p_{1} V_{1}^{n}=p_{2} V_{2}^{n}$, where $p_{2}=0.4 p_{1}$ and $V_{2}=2 V_{1}$.

Taking the logarithm to the base 10 , we have

$$
\begin{aligned}
\log p_{1}+n\left(\log V_{1}\right) & =\log p_{2}+n\left(\log V_{2}\right) . \\
\therefore n & =\frac{\log p_{1}-\log p_{2}}{\log V_{2}-\log V_{1}}=\frac{\log 2.5}{\log 2}=\frac{0.3979}{0.3010}=1.32 .
\end{aligned}
$$

6. Entropy changes. Given 2 pounds of air at 14.7 pounds absolute pressure and $32^{\circ} \mathrm{Fahrenheit}$, find and plot the following entropy changes.

(a) constant pressure, temperature increases to $736^{\circ}$ absolute, Fahrenheit.

(b) constant volume, temperature increases to $736^{\circ}$ absolute.

(c) constant temperature, pressure decreases to $\left(\frac{2}{3}\right)(14.7)$ pounds.

(d) what is the entropy change in Problem 5 ?

Solution. By eq. (38),

$$
\phi_{2}-\phi_{1}=c_{p} \log _{e} \frac{\theta_{2}}{\theta_{1}}=(0.237)\left[2.303 \log \frac{736}{491}\right]=0.0961 \text {. }
$$

Since this change in entropy is per pound, the total change is 0.192 .

(b) By eq. (37), $\quad \phi_{2}-\phi_{1}=c_{v} \log _{e} \frac{\theta_{2}}{\theta_{1}}=(0.169)\left[2.303 \log \frac{736}{491}\right]=0.0684$.

The total change in two pounds of air is then 0.137 .

1 For all of the problems of this book slide rule results are sufficiently accurate. The slide rule has been used in all numerical work connected with these problems. 
(c) By eq. (38), $\phi_{2}-\phi_{1}=-\left(c_{p}-c_{v}\right) \log _{e} \frac{p_{2}}{p_{1}}=(0.237-0.169)[2.30 \log 1.5]=0.0276$. Or the change for 2 pounds is 0.0552 .

'These changes are referred to in Figure 14 by the marginal letters used above.

(d) By eq. (36), $\phi_{2}-\phi_{1}=c_{p} \log _{e} \frac{V_{2}}{V_{1}}+c_{v} \log \frac{p_{2}}{p_{1}}=2.30[0.237 \log 2+0.169 \log 0.4]$ or 0.0090 , and hence for 2 pounds 0.018 .

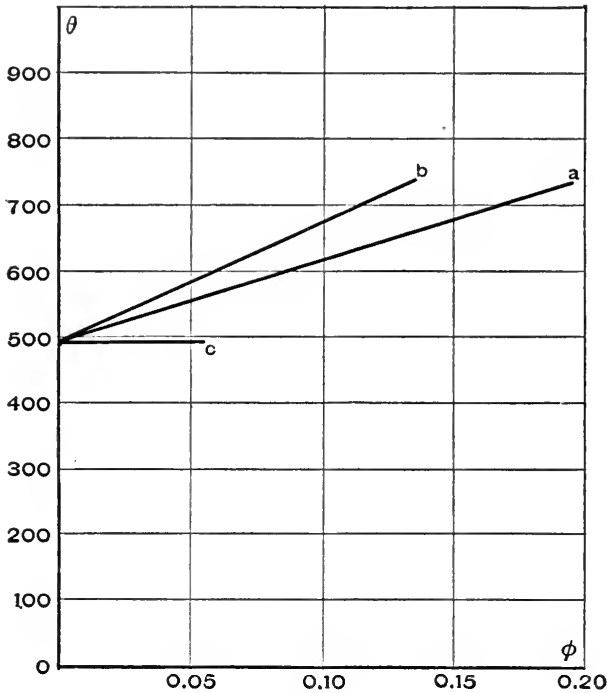

Fig. 14

7. Isothermal expansion. Three cubic feet of air at a pressure of 500 pounds absolute and a temperature of $450^{\circ}$ Fahrenheit expand isothermally to a volume of 18 cubic feet. Find the heat received. Find the work done per pound of air.

Solution. $\quad p_{1}=(500)(144), \quad v_{1}=3, \quad \theta_{1}=459.5+450=910, \quad v_{2}=18$.

By eq. (5), $\quad p_{2}=\frac{p_{1} V_{1}}{V_{2}}=\frac{(500)(3)}{(18)} 144=(83.3)(144){ }^{1}$

By eq. (18), $\quad W=(500)(144)(3)\left[2.30 \log \frac{18}{3}\right]=387,000$ foot pounds.

$$
\mathfrak{L}=\mathrm{AW}=\frac{387,000}{778}=498 \text { B. t. u. }
$$

By eq. (13),

$$
m=\frac{(500)(144)(3)}{(53.35)(910)}=4.46 \text { pounds. }
$$

Therefore work per pound is 87,000 foot pounds.

8. Adiabatic expansion. If the air in Problem 7 after reaching the volume of 18 cubic feet is allowed to expand adiabatically until

1 It is convenient to solve here for $p_{2}$, which will be used in Problem 9. 
the temperature is $200^{\circ}$ Fahrenheit, what is the amount of work done per pound of air?

Solution. Designate the state after adiabatic expansion by the subscript 3 .

$$
p_{2}=(83.3)(144), \quad V_{2}=18, \quad \theta_{2}=910, \quad \theta_{3}=459.5+200=660 .
$$

By eq. (32),

$$
\frac{V_{3}}{V_{2}^{\prime}}=\left(\frac{\theta_{3}}{\theta_{2}}\right)^{\frac{-1}{\kappa-1}} . \quad \therefore V_{3}=\left(\frac{910}{660}\right)^{\frac{1}{0.4}}(18)=40.2 .
$$

By eq. (19),

$$
W=\frac{p_{2} V_{2}}{\kappa-1}\left[1-\left(\frac{V_{2}}{V_{3}}\right)^{\kappa-1}\right]=\frac{p_{2} V_{2}}{\kappa-1}\left[1-\frac{\theta_{3}}{\theta_{2}}\right] \text {. }
$$

Therefore

$$
W=18 \frac{(83.3)(144)}{1.4-1}\left[1-\frac{660}{910}\right]=148,500 \text { foot pounds. }
$$

The work per pound is $\frac{148,500}{4.46}=33,300$ foot pounds.

9. Simultaneous equations in $p$ and $v$. If the expansions of Problems 7 and 8 are two transformations in a Carnot cycle, find the $p$ and $v$ coördinates of the four intersections of isothermals and adiabatics. What is the efficiency? Plot the cycle.

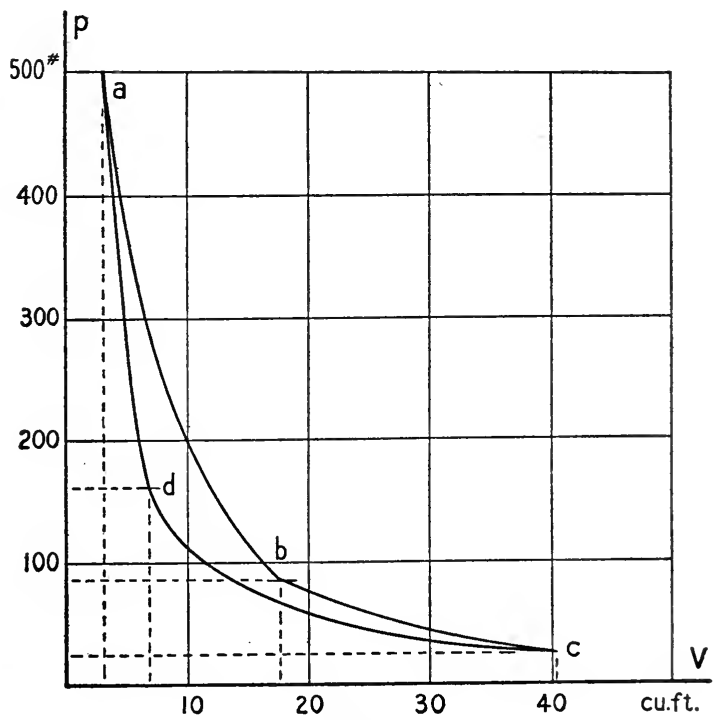

Fig. 15

Solution. From Problems 7 and 8 ,

$$
\begin{array}{lll}
p_{1}=500 & V_{1}=3 & \theta_{1}=910 \\
p_{2}=83.3 & V_{2}=18 & \theta_{2}=\theta_{1} \\
& V_{3}=40.2 & \theta_{3}=660
\end{array}
$$

Find $p_{3}$ by eq. (22).

$$
p_{3}=\frac{V_{2}{ }^{\kappa}}{V_{3}{ }^{*}} p_{2}=\left(\frac{18}{40.2}\right)^{1.40}(83.3)=27.1 \text { pounds per square inch. }
$$


Find $V_{4}$ by solving simultaneously the equations for the adiabatic through state point 1 and the isothermal through state point 3 , thus

$$
p_{4} V_{4}^{\kappa}=p_{1} V_{1}^{\kappa} \text { and } p_{3} V_{3}=p_{4} V_{4} .
$$

Therefore

$$
V_{4}=\left(\frac{p_{1} V_{1}^{\kappa}}{p_{3} V_{3}}\right)^{\frac{1}{\kappa-1}}=\left(\frac{(500)(144)(3)^{1.40}}{(27.1)(144)(40.2)}\right)^{\frac{1}{0.4}}=6.7,
$$

and $p_{4}=\frac{p_{3} V_{3}}{V_{4}}=\frac{(27.1)(144)(40.2)}{(6.7)}=(163)(144)$, or 163 pounds per square inch.

The values of $p, V$, and $\theta$ not given in Problems 7 and 8 are then

$$
\begin{array}{ll}
p_{3}=27.8 & V_{4}=6.7
\end{array} \theta_{4}=\theta_{3}
$$

The efficiency, by eq. (25), is $\eta=\frac{910-660}{910}=27.5$ per cent.

The plot of this cycle is seen in Figure 15 .

10. Pressure-volume plot. A quantity of air occupies 8 cubic feet at $70^{\circ}$ Fahrenheit and under an atmospheric pressure of 15 pounds. It is compressed adiabatically to a volume of 2 cubic feet, cooled at constant volume to its original temperature, and allowed to expand adiabatically to atmospheric pressure. It then heats at constant pressure to its initial temperature. Draw the pressure-volume plot of this cycle.

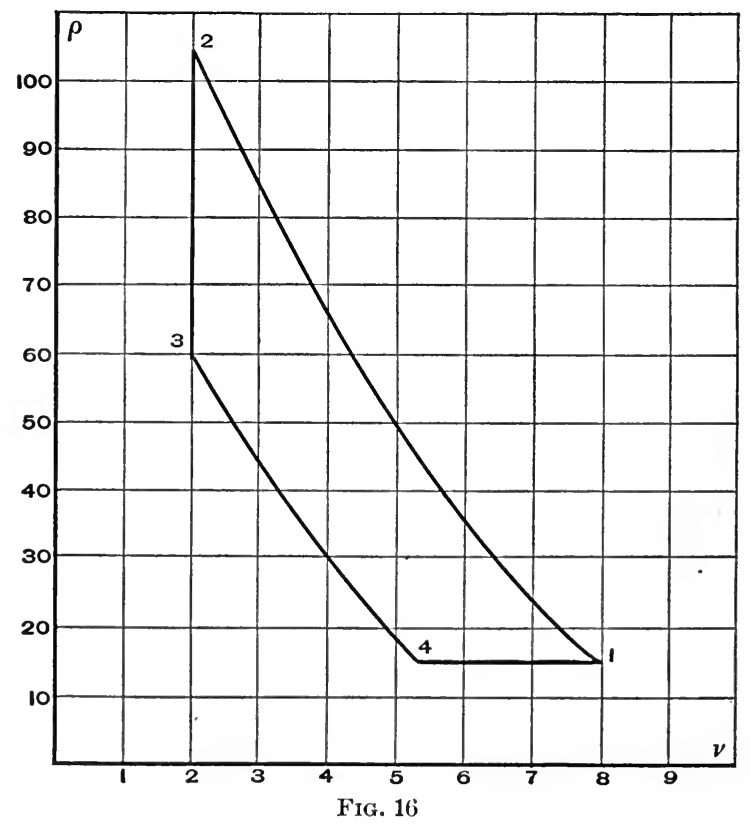

Solution. The $p-v$ plot is shown in Figure 16, which has been drawn by the use of the values given below. 
Given $p_{1} V_{1}{ }^{\kappa}=p_{2} V_{2}{ }^{\kappa}$, where $p_{1}=(15)(144), V_{1}=8, \theta_{1}=460+70=530, \quad V_{2}=2$, $\kappa=1.40$; then

$$
p_{2}=\left(\frac{8}{2}\right)^{1.40}(15)=104.6 \text { pounds per square inch. }
$$

By eq. (31), $\quad \theta_{2}=\left(\frac{V_{1}}{V_{2}}\right)^{\kappa-1} \theta_{1}=\left(\frac{8}{2}\right)^{0.4} 530=922$.

Since $\theta_{3}=530, \quad p_{3}=\frac{\theta_{3}}{\theta_{2}} p_{2}=\frac{530}{922} 104.6=60$.

During adiabatic expansion to atmospheric pressure

$$
p V^{\kappa}=p_{3} V_{3}^{\kappa}=p_{4} V_{4}^{\kappa} \text { and } p_{4}=15 .
$$

Hence

$$
V_{4}=\left(\frac{p_{3}}{p_{4}}\right)^{\frac{1}{\kappa}} V_{3}=\left(\frac{60}{15}\right)^{0.71}(2)=5.35 \text {, }
$$

and

$$
\theta_{4}=\left(\frac{2}{5.35}\right)^{0.4} \cdot(530)=358^{\circ} \text { absolute, or }-102^{\circ} \mathrm{F} .
$$

11. Regenerative cycle, Sterling engine. The Sterling hot-air engine works upon the following cycle (as shown in the $\theta-\phi$ plot of Fig. 17). From $a$ to $b$ the air in the engine expands isothermally at a temperature $\theta_{1}$, from $b$ to $c$ this air is cooled at essentially constant volume to the temperature $\theta_{3}$ and pressure $p_{3}$, from $c$ to $d$ it is then compressed practically isothermally to the volume $v_{1}$ and a pressure $p_{4}$, from $d$ to $a$ the air is heated at constant volume-to its initial condition.

This is accomplished by the use of a displacement piston $D$ in conjunction with a working piston $W$, as is shown in the diagram of Figure 18. The displacement piston, actuated by a rocker arm, moves up its full stroke during $d a$, while the working piston is essentially at rest. It

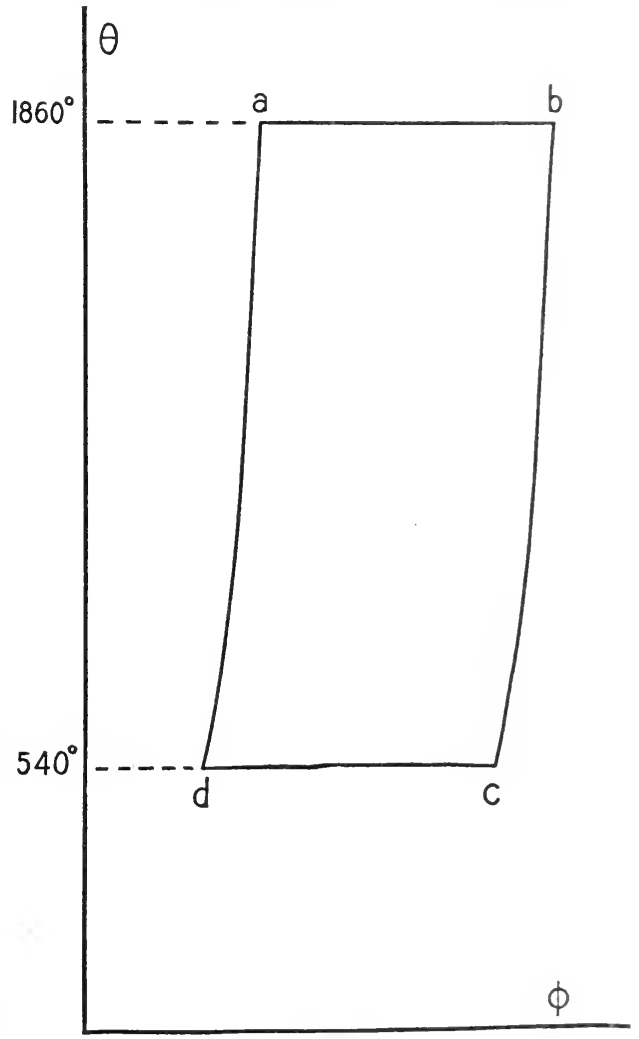

FIG. 17 
thus forces all the cool air at temperature $\theta_{3}$ through the regenerator $R$ into the lower portion beneath which there is a fire $F$. The re-

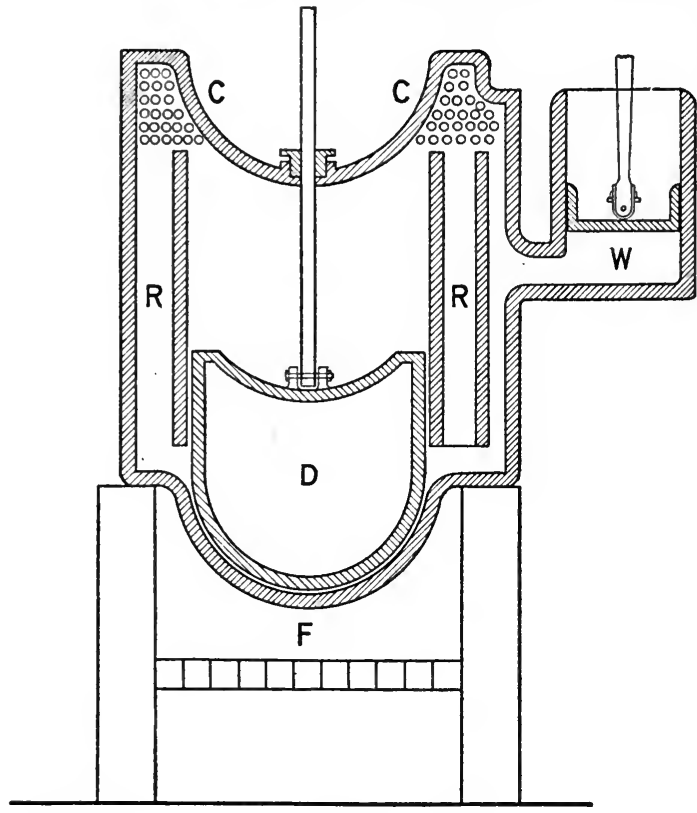

FIG. 18

generator consists merely of thin metal plates or wires which will rapidly absorb or reject heat. The air in contact now with the heating surface expands from $a$ to $b$ and causes a working stroke of the piston $W$, while the piston $D$ remains at the top of its stroke. When $W$ has reached the end of its stroke, the piston $D$ moves quickly down, thus causing the air below it to pass through the regenerator. The air passing through the regenerator gives up

heat and then passes into the refrigerator $C$. The pressure is thereby reduced from $b$ to $c$. The refrigerator consists of a set of pipes through which flows cooling water. The working piston $W$ now returns, compressing essentially isothermally at the temperature of the refrigerator all the air originally contained by both cylinders. This last transformation is represented by $c d$.

Neglecting clearance, find the efficiency for such an engine working between $1400^{\circ}$ and $80^{\circ}$ Fahrenheit. Assume the volume of the working cylinder to be 0.9 cubic foot and the entire volume, exclusive of the working cyclinder, to be 3 cubic feet. Compare with the Carnot engine in efficiency. Sketch the temperature-entropy plot and compare with the plot for the Carnot cycle. The lowest pressure is 16 pounds per square inch.

Solution. Denote by the subscripts $1,2,3$, and 4 the state points $a, b, c$, and $d$, respectively.

$$
\begin{array}{rlrl}
\theta_{1} & =\theta_{2}=460+1400=1860 & & \theta_{3}=\theta_{4}=460+80=540 \\
V_{1} & =V_{4}=3 & V_{2}=V_{3}=3+0.9=3.9 \\
p_{3} & =16 & &
\end{array}
$$


Then

$$
\begin{aligned}
& p_{4}=\frac{p_{3} V_{3}}{v_{4}}=\frac{3.9}{3} 16=20.8, \\
& p_{1}=\frac{\theta_{1}}{\theta_{4}} p_{4}=\frac{1860}{540} 20.8=71.6, \\
& p_{2}=\frac{\theta_{2}}{\theta_{3}} p_{3}=\frac{1860}{540} 16=55.1 .
\end{aligned}
$$

By eq. (18),

$$
\mathfrak{\Omega}_{1-2}=\frac{p_{1} V_{1}}{778} \log _{e} \frac{V_{2}}{V_{1}}=\frac{(71.6)(144)(3)}{(778)}\left[2.30 \log \frac{3.9}{3}\right]=10.42 \text { I3.t.u. }
$$

$\mathfrak{D}_{2-3}=\mathfrak{Q}_{4-1}$, since they are constant volume changes between the same limiting temperatures.

$$
\mathfrak{Q}_{3-4}=\frac{p_{3} V_{3}}{778} \log _{e} \frac{V_{4}}{V_{3}}=\frac{(16)(144)(3.9)}{778}\left[2.30 \log \frac{3}{3.9}\right]=3.03 \text { B.t. u. }
$$

The efficiency is then

$$
\eta=\frac{10.42-3.03}{10.42}=70.8 \text { per cent. }
$$

The Carnot efficiency is

$$
\eta=\frac{1860-540}{1860}=70.8 \text { per cent. }
$$

Discussion. This is an illustration of a regenerative cycle where, although the changes are at constant volume instead of isentropic, the efficiency is the same. There are other cycles possible which have the same efficiency as the Carnot for the same range of temperatures, but none has a higher efficiency, as is explained in Section 34.

12. Air compressor with and without clearance. An air compressor with perfect valves receives air at the atmospheric pressure of 15 pounds per square inch and delivers it at a constant pressure of 90 pounds per square inch. The compression follows

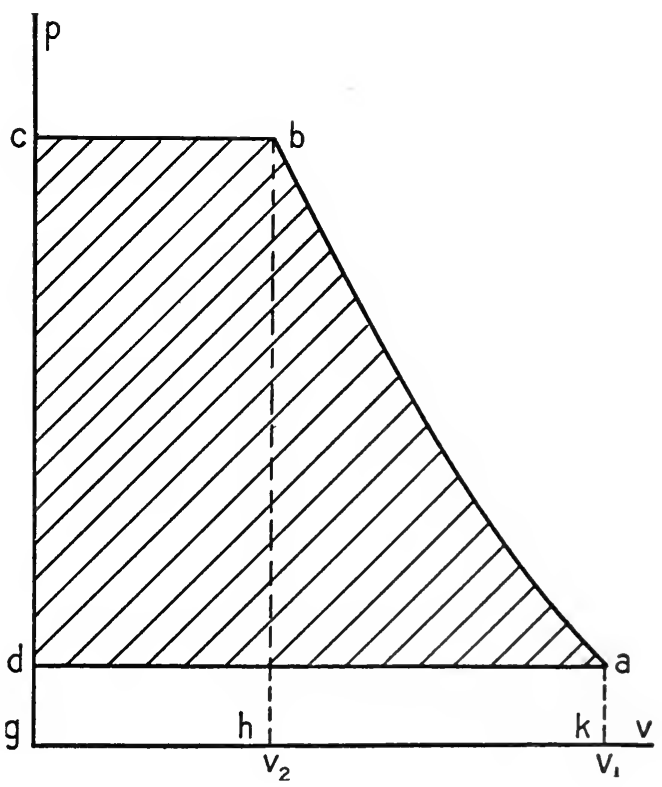

FIG. 19 the equation $p V^{n}$ equal to a constant where $n$ is 1.3 due to partial cooling. Draw the $p-v$ plot and find how many foot pounds of 
work are required for each cubic foot of air at the atmospheric conditions under which the condenser is working. (a) Neglect clear-

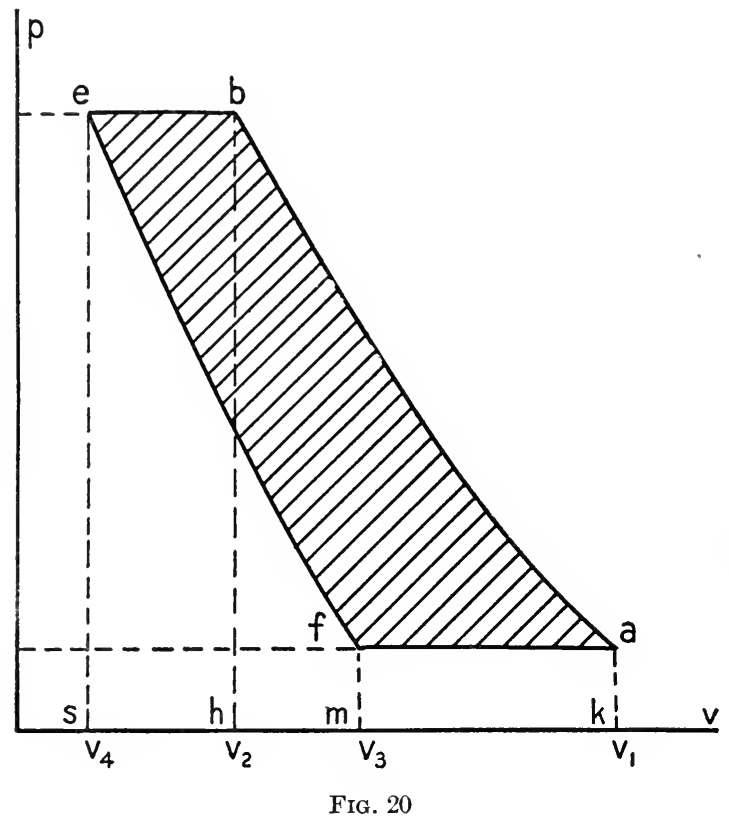
ance. (b) Allow for a clearance of 11.1 per cent of the piston displacement.

Solution. Representing the total piston displacement and clearance by $V_{1}$, the rolume after compression by $V_{2}$, the clearance volume by $V_{4}$, and the volume occupied by the clearance air at atmospheric pressure by $V_{3}$, Figures 19 and 20 represent respectively the cycle and the work done upon the air for conditions (a) and (b) above. The calculations are tabulated below for these two cases. It is important to notice that the net work done on the air is only that done in compressing it above the 15 pounds at which it is received.

(a) Without clearance.

Work of compression. By eq. (19), area $a b h k=3650 V_{1}$ Volume expelled at $90 \mathrm{lb}$. pressure, $V_{2}=\left(\frac{15}{90}\right)^{\frac{1}{1.3}} V_{1}=0.251 V_{1}$ Work of expulsion $(90)(144)\left(V_{2}-V_{4}\right)$, area $b c g h=3250 V_{1}$ Total work,

Work done by atmosphere, $6900 V_{1}$ Work done by clearance air. $\left(V_{3}=0.397 V_{1}\right)$, By eq. (15) Therefore net work of compressor, $4740 V_{1}$ Cubic feet of air (at atmospheric pressure) delivered, $\quad V_{1}$ Foot pounds of work per cubic foot delivered, 4740 (b) With clearance. area $a b h k=3650 V_{1}$ $V_{2}-V_{4}$

$$
\begin{aligned}
& =(0.251-0.100) V_{1} \\
& =0.151 V_{1}
\end{aligned}
$$

area besh $=1960 V_{1}$ $5610 V_{1}$ area $a f m k=1.300 V_{1}$ area efms $=1460 V_{1}$ $2850 V_{1}$ $0.603 V_{1}$ 4740

Discussion. The effect of the clearance in a frictionless compressor is not to increase the work per cubic foot of air delivered, but to require a larger piston displacement to deliver the same amount of air per stroke.

13. Otto gas engine cycle. The Otto, or de Rochas, cycle for a gas engine consists thermodynamically, in the ideal case, of the following processes : (1) adiabatic compression of an explosive mixture 
of air and gas; (2) heating at constant volume by combustion; (3) adiabatic expansion of the products of combustion to the original volume occupied by its constituents; (4) cooling at constant volume.

This cycle will be discussed upon the following assumptions: (1) the specific volumes of the constituents will be considered equal to that of the products of combustion under the same conditions of temperature and pressure ; (2) the products of combustion are considered to follow the laws of perfect gases and to have specific heats in the same ratio; (3) the density of the gases before and after combustion are considered equal to that of dry air under the same conditions of pressure and temperature.

The $p-v$ plot for such a cycle is shown in Figure 21. The piston makes four strokes for each working stroke or explosion. During the forward stroke $a b$ the piston draws into the cylinder a charge of gas and air. From $b$ to $c$ it compresses. From $c$ to $d$ the heating takes place instantaneously, due to the explosion of the mixture. During the forward stroke de external work is done. The valve then opens at $e$ and the gases exhaust, the pressure and temperature dropping to that of the atmosphere or the "muffler." In effect it is as if the valves had not opened but the gas had been cooled at constant volume as represented by the line $e b$. The fourth stroke now expels this gas against atmospheric pressure and is represented

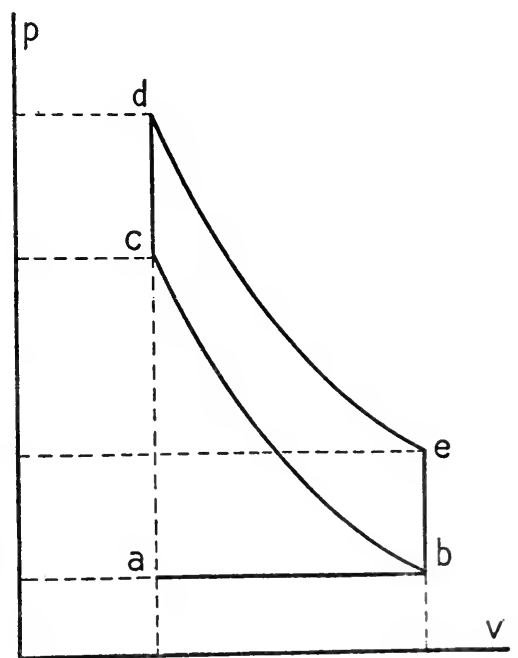

FIG. 21 by $b a$.

(a) If the clearance is 25 per cent of the piston displacement, find the theoretical thermal efficiency.

(b) If a mixture of gas and air which will develop 85 B. t. u. by combustion is taken into the cylinder at a pressure of 14 pounds and a temperature of $60^{\circ}$ Fahrenheit, find the temperatures at $c$ and $d$ and the pressures at $c, d$, and $e$.

(c) Compare the efficiency with the Carnot efficiency for the range of temperatures found from $(b)$. 
Solution. (a) All the heat taken in is along $c d$ in Figure 21. Similarly, the heat rejected is along $e b$. The calculation will be made for one pound of the explosive mixture.

The efficiency is $\quad \eta=\frac{\mathfrak{q}_{1}-\mathfrak{q}_{2}}{\mathfrak{q}_{1}}$,

where

$$
\mathfrak{q}_{1}=c_{v}\left(\theta_{d}-\theta_{c}\right) \text { and } \mathfrak{q}_{2}=c_{v}\left(\theta_{e}-\theta_{b}\right) \text {. }
$$

Hence

$$
\eta=\frac{c_{v}\left(\theta_{d}-\theta_{c}\right)-c_{v}\left(\theta_{e}-\theta_{b}\right)}{c_{v}\left(\theta_{d}-\theta_{c}\right)}=1-\frac{\theta_{e}-\theta_{b}}{\theta_{d}-\theta_{c}} .
$$

Since $d e$ and $c b$ are adiabatics, they must differ by a constant quantity of entropy. Equating then the difference in entropy found by eq. (3i) between $c$ and $d$ to that found between $e$ and $b$, gives

$$
c_{v} \log \frac{\theta_{e}}{\theta_{b}}=c_{v} \log \frac{\theta_{d}}{\theta_{c}}, \text { hence } \frac{\theta_{e}}{\theta_{b}}=\frac{\theta_{d}}{\theta_{c}} .
$$

Therefore

$$
\frac{\theta_{e}-\theta_{b}}{\theta_{d}-\theta_{c}}=\frac{\theta_{b}}{\theta_{c}}
$$

But since $b$ and $c$ lie on the same adiabatic, eq. (32) gives $\frac{\theta_{b}}{\theta_{c}}=\left(\frac{v_{c}}{v_{b}}\right)^{\kappa-1}$. Now $v_{c}$ is clearance and $v_{b}$ is piston displacement plus clearance. Hence

$$
\eta=1-\frac{\theta_{b}}{\theta_{c}}=1-\left[\frac{\text { clearance }}{\text { clearance }+ \text { piston displacement }}\right]^{\kappa-1} \text {. }
$$

Substituting for the clearance 25 per cent of the piston displacement gives

$$
\eta=1-\left[\frac{0.25}{1+0.25}\right]^{1.4-1}=1-0.525=47.5 \text { per cent. }
$$

(b) $p_{a}=p_{b}=14 . \quad \theta_{b}=60+460=520 . \quad \mathfrak{q}_{1}=85$ B. t. u.

$$
\begin{aligned}
& \theta_{c}=\theta_{b}\left(\frac{v_{b}}{v_{c}}\right)^{\kappa-1}=520\left(\frac{1}{0.525}\right)=990 . \\
& \mathfrak{q}_{1}=c_{v}\left(\theta_{d}-\theta_{c}\right)=0.169\left(\theta_{d}-990\right)=85 \text { B. t. u. }
\end{aligned}
$$

Therefore

$$
\theta_{d}=1490 \text {. }
$$

By eq. (32),

$$
p_{c}=\left(\frac{v_{b}}{v_{c}}\right)^{\kappa} p_{b}=(9.52)(14)=133
$$

Then

$$
\frac{p_{d}}{p_{c}}=\frac{\theta_{d}}{\theta_{c}} ; \text { therefore } p_{d}=\frac{1490}{990} 133=201 .
$$

By eq. (32),

$$
p_{e}=\left(\frac{v_{d}}{v_{e}}\right)^{\kappa} p_{d}=\frac{201}{9.52}=21 \text {. }
$$

(c) The Carnot efficiency is

$$
\eta=\frac{\theta_{d}-\theta_{b}}{\theta_{d}}=\frac{1490-520}{1490}=65 \text { per cent. }
$$

14. Diesel internal combustion cycle. Gas engines operating upon the Diesel cycle compress air to a high temperature, as shown by $b c$ in Figure 22. The temperature is sufficient to ignite the fuel, which is injected from $c$ to $d$ at a rate such that $c d$ is essentially an isothermal. Considering the initial compression to be adiabatic and from 15 pounds atmospheric pressure and $60^{\circ}$ Fahrenheit to 500 
pounds gauge, the isothermal to be for 15 per cent of the working stroke, and the remainder of the expansion to be adiabatic, solve for the following: (1) the temperature of the isothermal, (2) the clearance in per cent of the piston displacement, (3) the B.t.u. supplied by the combustion per pound of working substance, (4) the thermal efficiency, and (5) the Carnot efficiency for the same range of temperatures.

Solution.

(1) $\quad \theta_{c}=\left(\frac{p_{c}}{p_{b}}\right)^{\frac{\kappa-1}{\kappa}} \theta_{b}=\left(\frac{515}{15}\right)^{\frac{0.40}{1.40}}$ $=1430^{\circ}$ abs. or $970^{\circ} \mathrm{F}$.

(2) $\frac{v_{c}}{v_{b}}=\left(\frac{p_{b}}{p_{c}}\right)^{\frac{1}{\kappa}}$; therefore $v_{c}=0.08 v_{b}$ or $v_{c}=0.087\left(v_{b}-v_{c}\right)$.

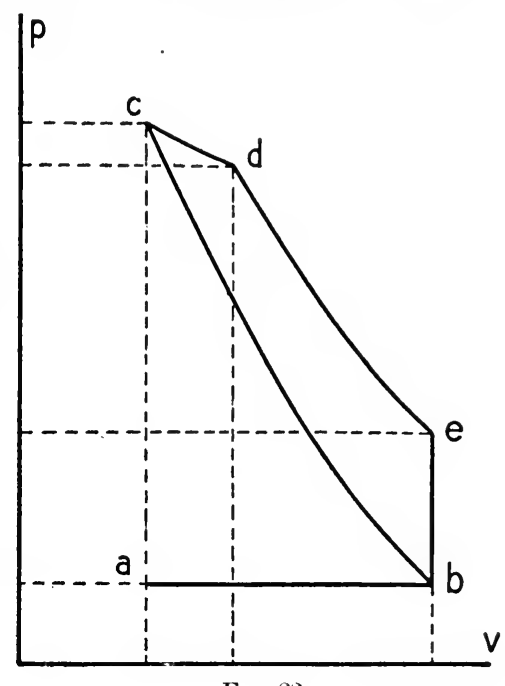

FIG. 22

That is, the clearance is 8.7 per cent of the piston displacement.

(3) Since

$$
v_{d}=0.15\left(v_{b}-v_{c}\right)+v_{c}=2.73 v_{c},
$$

the heat supplied along the isothermal is per pound of mixture

$$
\mathfrak{q}_{1}=\frac{p_{c} v_{c}}{778} \log _{e} \frac{v_{d}}{v_{c}}=\frac{R \theta_{c}}{778} \log _{e} \frac{v_{d}}{v_{c}}=\frac{(53.35)(1430)}{778}=98 \text { B.t.u. }
$$

Since

$$
\theta_{e}=\left(\frac{v_{d}}{v_{e}}\right)^{\kappa-1} \theta_{d}=\left[\frac{(2.73)\left(0.08 v_{b}\right)}{v_{b}}\right]^{0.40} 1430=780,
$$

the heat rejected at constant volume is

$$
\mathfrak{q}_{2}=0.169(780-520)=44 \text { B.t.u. }
$$

(4) Therefore the efficiency

$$
\begin{aligned}
& \eta=\frac{98-44}{98} \fallingdotseq 55 \text { per cent. } \\
& \eta=\frac{1430-520}{1430} \fallingdotseq 64 \text { per cent. }
\end{aligned}
$$

(5) The Carnot efficiency is

15. Air refrigeration. The fact that air falls in temperature during an adiabatic expansion is made use of in the design of air. refrigerating machines. The essential features of such a machine are shown in Figure 23. The air is received by the working cylinder $W$ from the room which is to be kept cool. Let its temperature and pressure be $\theta_{a}$ and $p_{a}$ respectively. The air is compressed adia- 
batically as shown in the $p$ - $v$ plot of Figure 24 . It is then cooled at constant pressure by passing it through a coil placed in a tank sup-

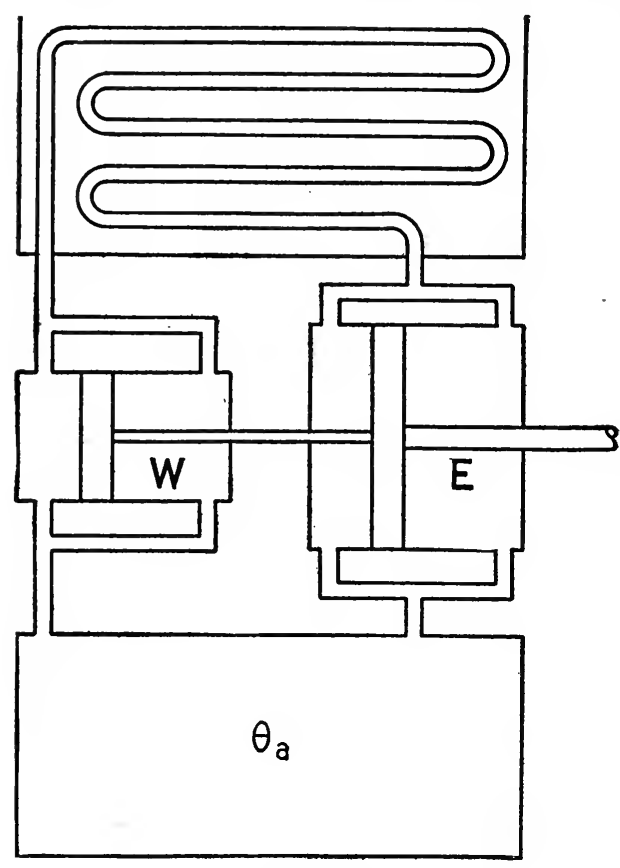

Fig. 23 plied with cooling water. After this it expands adiabatically along $c d$ as shown in the plot, and does work in the expansion cylinder $E$, which returns it to the prime mover, which is driving the compressor. The temperature of the air is now considerably below that of the room from which it was received. It then enters the room and is heated at constant pressure.

If $p_{a}$ is 14.7 pounds absolute, $p_{b}$ is 14 times $p_{a}, \theta_{a}$ is to be zero degrees Fahrenheit, how much heat is abstracted from each pound of air while it is in the cooling room? Sketch the $p$ - $v$ plot for each cylinder and the $\theta-\phi$ plot for the entire cycle. Neglect the clearance. Assume that the cooling water reduces the temperature of the air to $95^{\circ}$ Fallrenheit.

Solution.

$p_{b}=14 p_{a}=206$ pounds per square inch。

Calling $v_{a}$ equal to $v$, we find $v_{b}$ and $\theta_{b}$ by eq. (32) and (19) as follows :

$$
\begin{aligned}
& v_{b}=\left(\begin{array}{l}
p_{a} \\
p_{b}
\end{array}\right)^{\frac{1}{\kappa}} v_{a}=\left(\frac{1}{14}\right)^{0.71} v=0.154 v, \\
& \theta_{b}=\left(\frac{p_{b}}{p_{a}}\right)^{\frac{\kappa-1}{\kappa}} \theta_{a}=(14)^{0.29}(460)=990 .
\end{aligned}
$$

These values are plotted in Figure 25 , which shows the work of the working cylinder $W$.

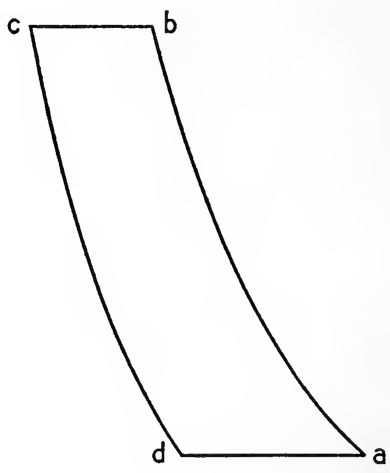

Fig. 24 
Similarly, since $\theta_{c}=95+460=555$ and $p_{c}=p_{b}$ and $p_{d}=p_{a}$,

$v_{c}=\frac{\theta_{c}}{\theta_{b}} v_{b}=\frac{555}{990}(0.154 v)=0.086 v$ $v_{d}=\left(\frac{p_{c}}{p_{d l}}\right)^{\frac{1}{\kappa}} v_{c}=(14)^{0.71}(0.086 v)=0.562 v$ $\theta_{d}=\left(\frac{p_{d}}{p_{c}}\right)^{\frac{\kappa-1}{\kappa}} \theta_{c}=\left(\frac{1}{14}\right)^{0.29}(555)=260$

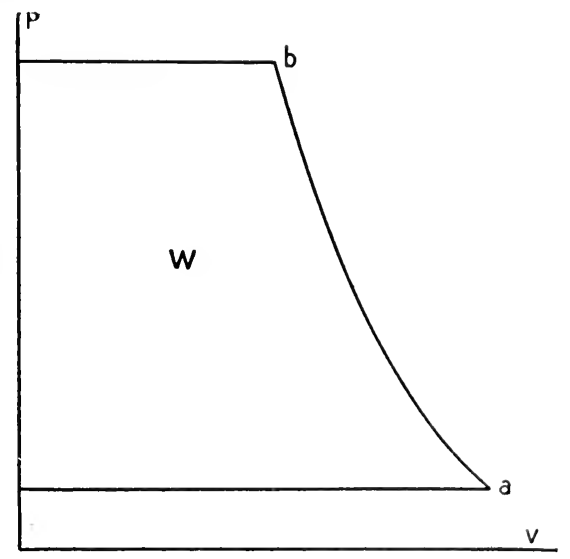

FIG. 25

These values are plotted in Figure 26, which shows the work done in the expansion cylinder $E$.

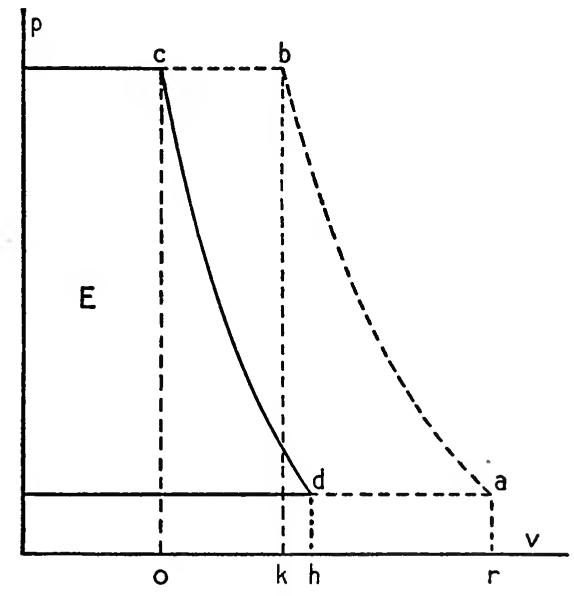

FIG. 26

The net input to the compressor is the dotted area $a b c d$ in Figure 26. This input will be found by parts as below :

$$
\begin{aligned}
& \text { area } a b k r=\frac{p_{b} v_{b}-p_{a} v_{a}}{\kappa-1}=(144)(14.7) \frac{(14)(0.154)-1}{0.40} v=6100 v \text { foot pounds. } \\
& \text { area } b c o k=p_{b}\left(v_{b}-v_{c}\right)=(206)(144)(0.068) v=1990 v \text { foot pounds. } \\
& \text { area } c d h o=\frac{p_{c} v_{c}-p_{d} v_{d}}{\kappa-1}=(144)(14.7) \frac{(14)(0.086)-(0.562)}{0.40} v=3400 v \text { foot pounds. } \\
& \text { area } d a r h=p_{a}\left(\dot{v}_{a}-v_{d}\right)=(144)(14.7)(0.438) v=930 v \text { foot pounds. }
\end{aligned}
$$


The net input is then 3760 foot pounds per cubic foot of entering air.

One pound of air at the admission temperature and pressure has a specific volume of

$$
\frac{\theta_{a}}{491} 12.39=11.6 \text { cubic feet. }
$$

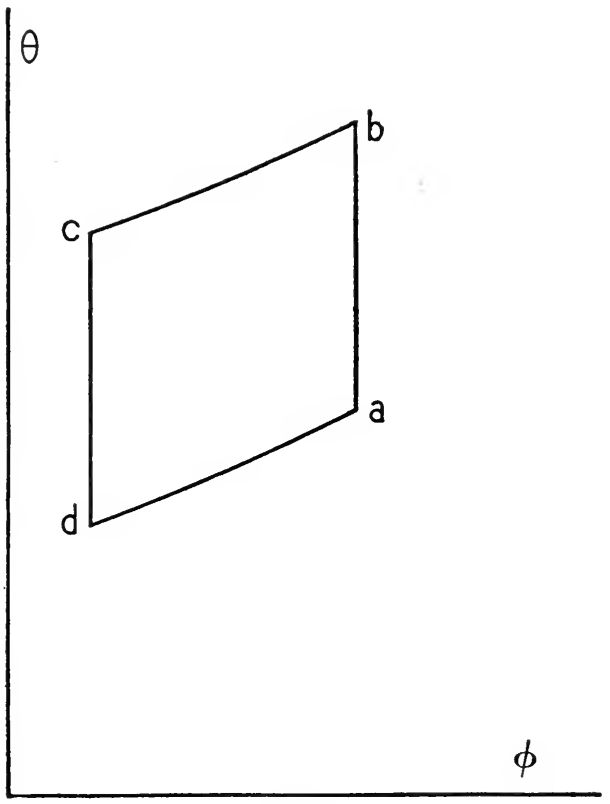

FIG. 27

The work of the compressor per pound of air pumped is then

$$
(3760)(11.6)=43,600 \text { foot pounds. }
$$

The B.t. u. equivalent of this work is

$$
\frac{43,600}{778}=56 \text { B.t.u. }
$$

The heat abstracted per pound by the room it is desired to refrigerate is

$$
c_{p}\left(\theta_{d}-\theta_{a}\right)=0.237(460-260)=47.5 \text { B.t.u. }
$$

The cooling water must then remove the sum of these two amounts of heat or 103 B.t.u. As a check calculate the heat abstracted by the cooling water. It is eviclently

$$
c_{p}\left(\theta_{b}-\theta_{c}\right)=0.237(990-555)=103 \text { B.t.u. }
$$

The temperature-entropy plot is shown in Figure 27. 


\section{CHAPTER III}

\section{WATER AND ITS SATURATED VAPOR}

44. Phenomena of Vaporization. In accordance with the kinetic theory the molecules of a liquid are considered to be in motion in a manner similar to the molecules of a gas, except for the fact that the average free path which a molecule travels between impacts is very much less in a liquid, and except for the fact that the molecules are now within the sphere of each other's influence and exert large mutual attractions. The continual evaporation which takes place from the surface of an exposed liquid demands, however, the generally accepted assumption, that occasional molecules possessing individually kinetic energies higher than the average for the entire body convert a portion of their kinetic energy into potential energy by breaking away from the surface attraction of the neighboring molecules and passing into the space above the liquid. That this free evaporation is facilitated by an increase of temperature, that is, by an increase of molecular kinetic energy, is a fact of common knowledge which supports this theory.

If the space above the liquid is enclosed, some of the molecules, which have escaped, and in that space are moving essentially after the manner of gaseous molecules, will in the course of their motion impinge upon the surface of the liquid and once more become a part of it. When, however, there are as many molecules entering the liquid in this way as leave it in the same time, there is a so-called "state of active equilibrium," and the vapor constituted by the free molecules is said to be saturated. If now the volume above the liquid, which these free molecules occupy, is reduced, there will be momentarily a larger density, and hence a larger number of impacts of the free molecules with the liquid, and hence a condensation of the vapor. The ultimate result will be a reduction of the number of free molecules until the density is reduced to its former value and the state of active equilibrium is again attained. Other things being equal, the density of a vapor in contact with its liquid is independent of the volume which the vapor is free to oceupy. 
On the other hand, if the temperature of the liquid is increased, the equilibrium will be momentarily disturbed by the escape from the liquid of a larger number of molecules than return to it in the same time. This momentary disturbance lasts until a new stable density of greater value is reached. The density of a saturated vapor in contact with its liquid is then some function of its temperature.

The pressure exerted by the vapor is also a function of the temperature, since by the kinetic theory the pressure depends upon the energy of the molecules and their number per unit volume. The number per unit volume is directly proportional to the density, and hence the pressure increases with a twofold rapidity as the temperature rises. The temperature at which the pressure of the saturated vapor would be equal to the external pressure upon the surface of a given body of liquid is called the boiling temperature of the liquid for that condition of pressure. At this temperature bubbles of the vapor start to form beneath the surface of the liquid, rise to the top, and burst into vapor. After boiling commences no further increase in the temperature (for constant pressure) is possible until all the liquid has been converted into vapor.

Water vapor, or steam, as it is usually called, is colorless and transparent. The cloudy white form it frequently takes is due to the condensation of part or all of the original steam into minute drops of water. In this case the steam is called "wet" because of the mixture with it of water particles. Steam free from moisture, whether due to condensation, entrained water, or other causes, is called "dry."

'The temperature and pressure of water vapor' are always the same as the temperature and pressure of the water with which it is in contact. ${ }^{1}$ If, however, the saturated vapor is removed from contact with its liquid, its temperature may be raised above the original boiling point. The steam is then said to be superheated. If the pressure of the superheated steam is kept constant, its volume increases with the temperature; and also, if its volume is kept constant, the pressure increases with increased temperature. In steam engineering practice the steam is superheated in its passage from the boiler to the engine as the result of passing through coils of pipe in the flue of the boiler or as the result of some similar arrangement. Its volume per pound, or specific volume, then increases, and this increase is cared for at once by the motion of the piston of the engine. The

1 Except for differences beneath the surface of the liquid due to the hydrostatic pressure. 
effect is the same as if the steam were entirely separated from the water from which it is produced.

45. Specific Heat of Water. In order to convert a pound of water into steam, it is necessary, first, to raise the temperature of the water to that corresponding to the boiling point for the given pressure, and, second, to vaporize this water. In each case the necessary heat may be expressed by an equation of the form $d \mathfrak{Q}=A d S+A d I+A d W$, as in Section 8. In raising the temperature of the water there is produced a small increase in its volume. For ordinary engineering purposes this is so small that the terms $A d I$ and $A d W$ may be neglected. This necessary increase in the heat of the liquid will be represented by the symbols $q_{2}-q_{1}$, the significance of which will appear more fully later in this section.

This increase in the heat of the liquid may be found approximately by assuming the specific heat to be constant and of average value unity. Then the heat, $q_{2}-q_{1}$, required to change one pound of water from a temperature of $t_{1}$ to the boiling temperature $t_{2}$ is expressed as $q_{2}-q_{1}=t_{2}-t_{1}$. It is in general desirable to know this necessary heat more accurately, and this requires a knowledge of the specific heat of water at various temperatures. The curve of Figure 28 shows the variations of the value of the specific heat of

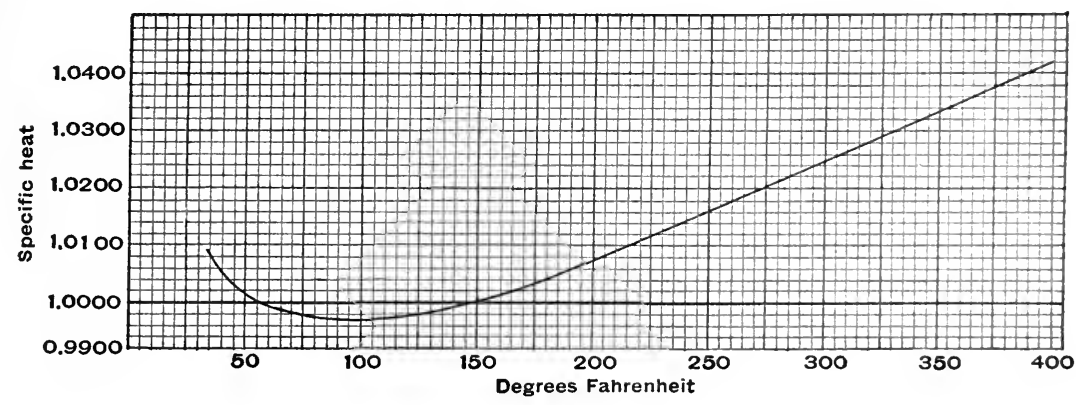

FIG. 28

water, taking the specific heat as unity for the change from $62^{\circ}$ to $63^{\circ}$ Fahrenheit. From such a curve, accurately construeted on a large scale, it would be possible to obtain the value of the specific heat for each degree and then by a summation of the form $\Sigma c d t$ to obtain $q_{2}-q_{1}$.

It is more convenient, however, to use some set of tables, in which are tabulated the results of such a summation from $32^{\circ}$ Fahrenheit, as a lower limit, to $t^{\circ}$ as an upper limit, where $t$ has for values all 
the whole degrees within the ordinary range of engineering work. The heat which is required to raise one pound of water from $32^{\circ}$ to $t_{1}^{\circ}$ will be represented by $q_{1}$. Similarly, the heat in B. t. u. required to raise one pound of water from $32^{\circ}$ to $t_{2}{ }^{\circ}$ is $q_{2}$. The heat required to raise one pound of water from $t_{1}$ to $t_{2}$ is then $q_{2}-q_{1}$ B.t. u. Such a table of values for $q$ is illustrated in Table 3 , on page 130, for a few chosen values. Certain other constants for steam, which will be discussed later, enter into this table.

The plot of Figure 29 shows the general nature of the relation between pressure and temperature for water vapor. It gives, in

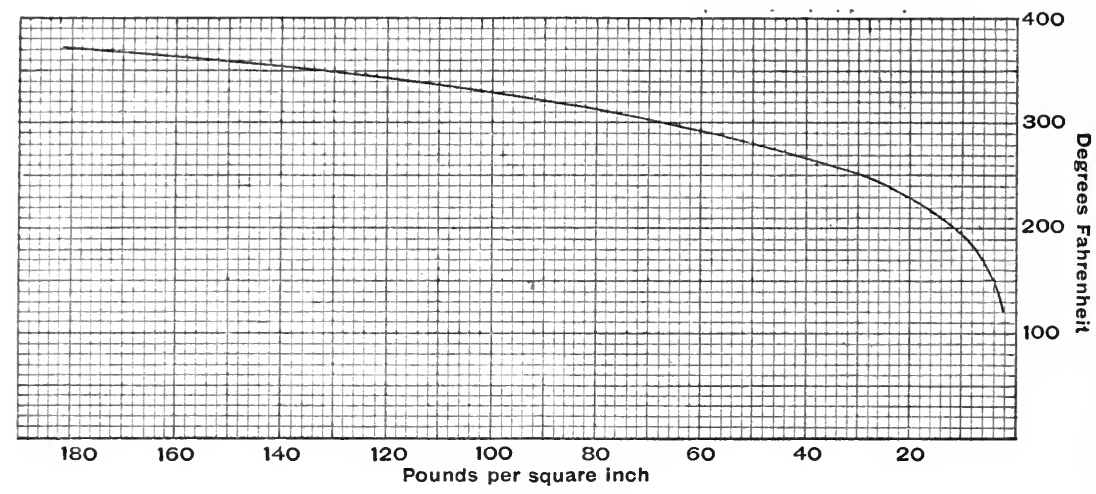

Fig. 29

other words, the boiling point temperature corresponding to a given pressure. There is to be noticed at the higher temperatures the rapid increase of pressure mentioned in Section 44. In Table 3, the value of $p$ corresponding to any given temperature $t$ is the pressure in pounds per square inch for which $t$ is the boiling point.

46. Heat of Vaporization. The heat required to vaporize one pound of water may be considered in three parts. The first of these, namely, $q_{2}-q_{1}$, which is required to raise the temperature of the water to the boiling point, has already been discussed. The remaining two have been considered partially in the illustration given on page 7 . They are $\rho$, the internal latent heat required to separate the molecules against their mutual attractions, and Apu, the external latent heat or heat equivalent of the external work which must be clone against the pressure $p$ to cause the increase, $u$, in the volume. This increase $u$ is $s-\sigma$, where $s$ represents the specific volume of one pound of dry steam at the pressure $p$, and $\sigma$ represents the volume of one pound of water. 
The total heat required to raise one pound of water from $32^{\circ}$ Fahrenheit to a temperature $t$ and there to vaporize all of it is then

where

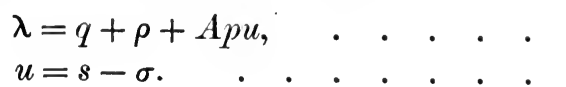

The letter $\lambda$ will be used hereafter as a general symbol for the total heat contents of a pound of water (in whatever form it may be) as measured by the heat in B.t.u. required to change the water from $32^{\circ}$ Fahrenheit to the condition for which $\lambda$ represents the heat contents. Also the letter $r$ will be used to denote the total latent heat of vaporization. Thus

$$
r=\rho+A p u \text {. }
$$

In Figure 30 is shown the general relation of $q, \rho, A p u, \lambda$, and $s$ for steam at various temperatures. It is to be noticed that the term $A p u$, which is represented in the figure by the portion of the ordinate between the curve of $\rho$, the "internal latent heat," and $r$, the total "latent heat," is almost constant. Since the specific volume of a pound of water is always approximately 0.016 cubic foot, it may for many purposes be entirely neglected as compared with the specific volume $s$ of dry steam. Hence the product $A p u$ is practically equal to Aps. This relation between $p$ and $s$ has been expressed in empirical formulas for calculating the specific volume of dry steam for any pressure. Thus by Zeuner as

and by Rankine as

$$
\begin{aligned}
& p s^{1.0646}=479, \quad . \quad . \quad . \quad . \quad . \quad . \quad . \\
& p s^{\frac{17}{16}}=482 .
\end{aligned}
$$

These equations obviously make allowance for the fact that $A p u$ is not exactly constant but increases somewhat at higher pressures.

The values for $p, r, \rho, A p u, s$, and $\gamma$, the density or mass per eubic foot, for selected values of $t$ are given in Table 3 on page $130 .{ }^{1}$ It - is to be remembered that these values of $r, \rho, A p u$, as well as $q$, are all in B.t.u. per pound. The specific volume $s$ is in cubic feet per pound. The pressure $p$ is in pounds weight per square foot, but since pressures are usually measured by gauges graduated in pounds

\footnotetext{
1 A convenient set of steam tables is the work of Professor Peabody of the Massachuset ts Institute of Technology. It is published under the title "Steam and Entropy Tables" by Wiley and Sons. The values used in this text and included in Table 3 are taken from the 8th edition by the kind permission of the anthor and the publishers. The symbols used in Table 3 are those of Professor Peabody except in the case of entropy, which is consiclered later in Sections 49 and 50. The tables compiled by Professors Marks and Davis of Harvard University also embody in a most convenient form the results of the recent investigations (Longmans, 1908).
} 
per square inch, the values of the table in the column headed by $p$ are so expressed and must be multiplied by a factor of 144 before being used in any numerical calculations.

It is to be noticed also from Figure 30 that $\rho$, the internal latent heat of vaporization, decreases with the volume. This is in accord-

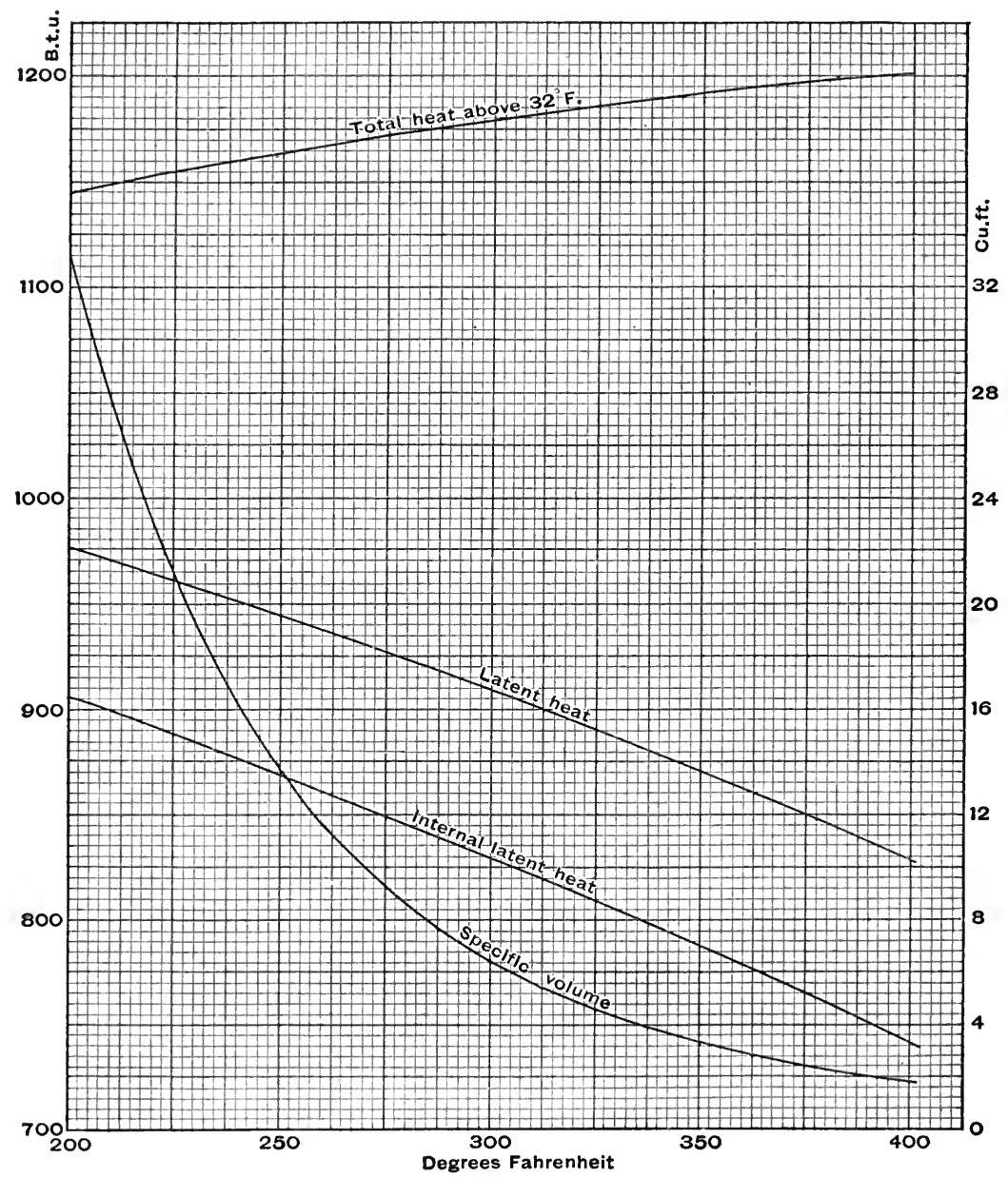

Fig. 30

ance with the kinetic theory, for when occupying a smaller volume the molecules are closer together and less work has been done in producing vaporization against their mutual attractions.

47. Quality of Steam. Consider a pound of water at $32^{\circ}$ Fahrenheit, and the results of raising its temperature to $t^{\circ}$ and there evap- 
orating against the corresponding pressure of $p$ pounds, not the entire pound of water as the problem has previously been considered, but a fraction of a pound, say $x$. Then obviously the fundamental equations given below on the left hand must be restated as shown on the right hand.

$$
\begin{gathered}
\lambda=q+r=q+\rho+A p u . \ldots(43) \quad \lambda=q+x r=q+x p+x A p u . . \\
v=s=u+\sigma . \ldots(44) \quad v=x s+(1-x) \sigma=x u+\sigma . .
\end{gathered}
$$

In general, if from any cause the steam considered is not dry but has mixed with it water, and the mixture is $x$ parts dry steam and $(1-x)$ parts water, then $x$ is defined as the "quality," and the modified equations given above must be used. The quality $x$ is usually expressed in per cent, but obviously it must enter into these equations as a decimal. The amount of water present, $(1-x)$ parts, is expressed as a per cent and called the "priming."

48. Intrinsic Energy of Steam. In the formation of one pound of dry steam at a pressure $p$, it is to be noted carefully that the molecules of the forming steam act as direct agents by which a certain amount of mechanical work, $p u$, is done at the expense of $A p u$ heat units supplied by the fire. At no time do these $p u$ units constitute part of the intrinsic energy of the steam. Therefore, in the equation $\lambda=q+\rho+A p u$, which is in the form of $\mathfrak{Q}=A(d S+d I+d W)$, the term $q+\rho$ represents the intrinsic energy of the steam and corresponds to $A d S+A d I$. Representing by $\epsilon$ the intrinsic energy of a pound of steam and water mixture, we have, if $x$ is unity,

$$
A \epsilon=q+\rho . \quad \text {. . . . . . }
$$

Or, more generally, for the intrinsic energy of one pound of a mixture of quality $x$,

$$
A \epsilon=q+x \rho . \text {. . . . . . . }
$$

49. Entropy of the Liquid. By definition, the entropy of a body in state 2 is the integral $\int_{1}^{2} \frac{d \mathfrak{q}}{\theta}$, taken along any reversible path from state point 1 , arbitrarily chosen as a zero of entropy. By convention for steam the point 1 is taken as the entropy of a pound of water at $32^{\circ}$ Fahrenheit. The heating of water is a reversible process, and the integral representing the entropy of one pound, at any temperature $t^{\circ}$ Fahrenheit or $\theta^{\circ}$ Fahrenheit on the absolute scale, may then be written as

$$
\phi_{w}=\int_{\theta=491.5}^{\theta} \frac{c d \theta}{\theta} . \quad . \quad . \quad . \quad . \quad . \quad .
$$


As an illustration, the entropy of water at $33^{\circ}$ Fahrenheit will be calculated. Thus

$$
\phi_{w}=\int_{491.5}^{492.5} c\left(\frac{d \theta}{\theta}\right)=\log _{e} \frac{492.5}{491.5}=0.0021 .
$$

By a proper allowance for the varying value of the specific heat $e$, the entropy of water may be calculated in this way for various temperatures. Values so calculated are given in Table 3 in the column headed $\phi_{w}$. This symbol will be used hereafter to represent the entropy of one pound of water.

50. Entropy of Vaporization. In a somewhat similar manner, since vaporization is a reversible process, the increase of entropy $\phi_{v}$ due to vaporization may be found. Thus

$$
\phi_{v}=\int \frac{d q}{\theta}=\frac{r}{\theta}, \quad . \quad . \quad . \quad . \quad . \quad . \quad .
$$

where the value of $\theta$ is the absolute temperature at which vaporization occurs, and $r$ is the heat, $\int d \mathfrak{q}$. As an illustration, the entropy of vaporization for $212^{\circ}$ Fahrenheit will be calculated. Thus, substituting for $r$ from Table 3 ,

$$
\phi_{v}=\frac{969.7}{459.5+212}=1.444 \text {. }
$$

The symbol $\phi_{v}$ will be used to represent the increase in entropy per pound of steam due to vaporization.

The total entropy of a pound of dry steam is then

$$
\phi=\phi_{w}+\phi_{v} \text {. }
$$

If the quality is not unity, but is $x$, then the total entropy of a pound of the mixture is

$$
\phi=\phi_{w}+x \phi_{v} \text {. . . . . . . . }
$$

51. Temperature-Entropy Chart for Steam. If the temperatureentropy cliagram for the change in entropy of one pound of water from $32^{\circ}$ Fahrenheit to some temperature $t_{1}{ }^{\circ}$ is plotted, it will appear as shown in Figure 31 by the line Fab. If the diagram is constructed for several higher temperatures, as $t_{2}, t_{3}$, and so on, it will be found that for these temperatures the changes in entropy from $32^{\circ}$ are represented by lines $F c d, F e f$, and so on. Such a temperature-entropy diagram for one pound of water when constructed for a sufficient number of temperatures is usually known as a "temperature-entropy chart for steam." It is of considerable value in the solution of problems in steam turbine study and of interest, although probably 
WATER VAPOR

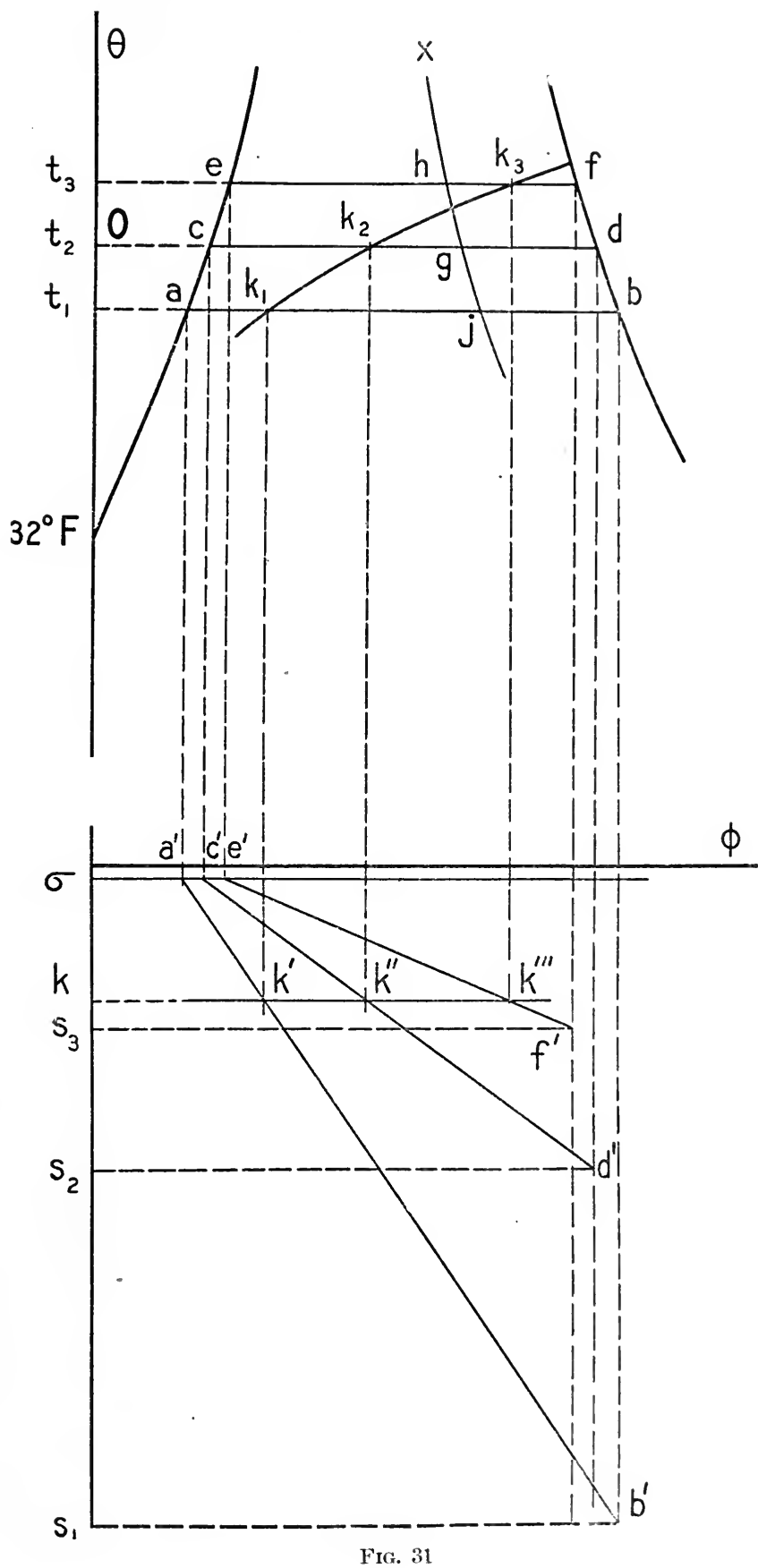


of lesser value, in the study of the steam engine. Such a temperature-entropy chart is given in part on page 86 .

The curve Face of Figure 31 is called the "water line," and the locus of the points like $b, d$, and $f$, is the "dry steam line" or "line of constant steam weight."

Since the entropy of a mixture is $\phi_{w}+x \phi_{v}$ and the length of the lines $a b, c d$, and $e f$ represents values of $\phi_{v}$, the entropy of any mixture may be easily determined from this chart. Thus if the entropy of a mixture of quality $x$ at a temperature $t_{2}$ is desired, we proceed as follows. Divide the line $c d$ into two parts by a point $g$ so located that $\mathrm{cg} / \mathrm{cd}$ shall equal $x$. Then obviously the length of the line $\mathrm{cg}$ is $x \phi_{v}$ and the total entropy of the mixture is represented by the line $O g$. For convenience in making such interpolations on the chart a series of lines like $h g j$ may be drawn, indicating all the points of the same quality for values of $x$ differing by 10 per cent, as is done in Figure 32. (In the chart on page 86 such lines have been drawn for differences of one per cent.) These lines of constant quality are usually called " $x$ lines."

Since the temperature is constant during vaporization, if the pressure is constant, the lines $a b, c d$, and so on represent constant pressure lines, the values of which correspond to the temperatures $t_{1}, t_{2}$, and so on.

Lines of constant volume showing the entropy of all states of a mixture of steam and water which occupy per pound the same volume may be drawn as follows. Continue the axis of temperatures below the entropy axis and let it represent to a convenient scale an axis of volumes. Draw a line parallel to the entropy axis and below it by an amount equal to $\sigma$, the specific volume of water. In Figure 31 the distance $\sigma$ is made disproportionately large for clearness. The letters $a^{\prime}, c^{\prime}$, and $e^{\prime}$ refer to the intersections of the dotted vertical projection lines with this constant volume line through $\sigma$. Then for any pressure and temperature as $t_{1}$ project normal to the entropy axis the line $a a^{\prime}$ to intersect the constant volume line $\sigma$ in $a^{\prime}$, and the line $b b^{\prime}$ to intersect the line $s_{1} b^{\prime}$, which is drawn through $s_{1}$, the point on the axis of volumes which corresponds to the specific volume of dry steam at the temperature $t_{1}$. The line $a^{\prime} b^{\prime}$ shows then the increase of entropy with increasing volume due to vaporization at this temperature. In a similar manner, other lines, showing the relations of volume and entropy for the temperatures $t_{2}$, $t_{3}$, and so on, may be drawn. 'These are represented by $c^{\prime} d^{\prime}$ and $e^{\prime} f^{\prime}$. For any volume as $k$ the line of constant volume $k k^{\prime \prime \prime}$ intersects the 
lines $a^{\prime} b^{\prime}, c^{\prime} d^{\prime}$, and $e^{\prime} f^{\prime}$ in points $k^{\prime}, k^{\prime \prime}$, and $k^{\prime \prime \prime}$, the abscissas of which represent the entropy of mixtures of constant volume $k$ but of different temperatures. The corresponding temperatures may be found by projecting the points $k^{\prime}, k^{\prime \prime}$, and $k^{\prime \prime \prime}$ back onto the lines $a b, c d$, and $e f$, respectively. The ordinates of these projections, namely $k_{1}, k_{2}$, and $k_{3}$, represent the temperatures for mixtures of volume $k$. The line $k_{1} k_{3}$ is then on the temperature-entropy plot a line of constant volume, and similarly, the lines $a^{\prime} b^{\prime}, c^{\prime} d^{\prime}$, and $e^{\prime} f^{\prime}$ on the volumeentropy plot are lines of constant pressure and temperature. On the chart of page 86 several constant volume lines are represented.

A family of curves for constant total heat contents might also be constructed through all the points for which $\lambda=q+x r=$ constant, for different values of the parameter $\lambda$. These lines of constant heat contents are of especial value in the solution by the use of a $\theta-\phi$ chart of problems on the flow of steam through nozzles. There is unfortunately no graphical method of constructing constant heat content curves, and the calculation and plotting of separate points is necessarily tedious. Constant heat curves are shown in the chart on page 86 . Similarly, curves for constant intrinsic energy might

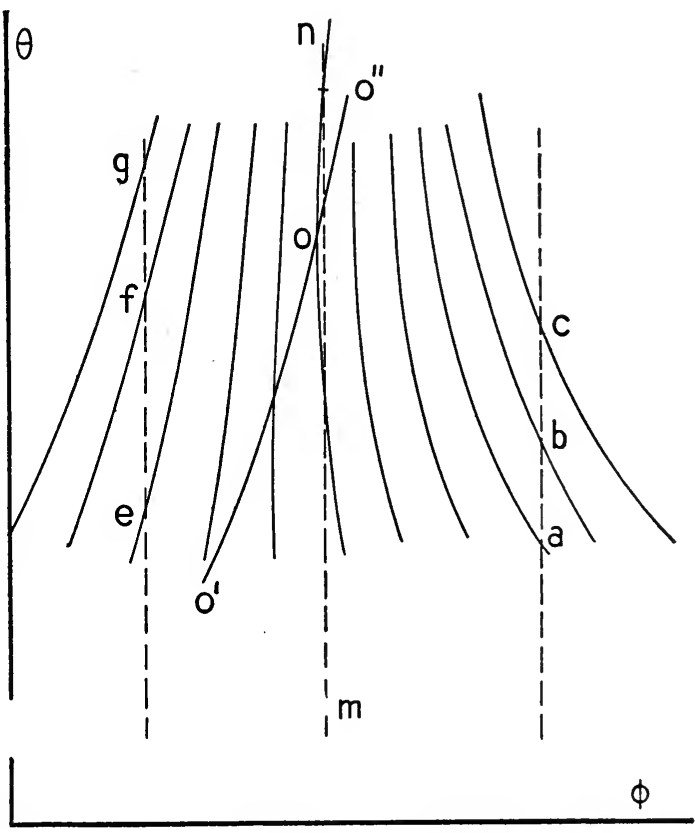

FIG. 32 be plotted in accordance with the relation $\epsilon=q+x \rho=$ constant. The general appearance of these curves is similar to those for constant heat contents, but slightly different in slope. ${ }^{1}$

52. Isentropic Transformation of a Mixture. From a study of the temperature-entropy diagram of Figure 32 it is evident that if an

1 For a method of determining graphically the intrinsic energy corresponding to a desired point on the $\theta-\phi$ chart, the reader is referred to Berry, "Temperature Entropy Diagram," 1st edition, pp. 28-29. For the converse problem there is no graphical solution. 
isentropic compression $a b c$ be made, starting with a mixture the state of which is represented by $a$, as successive lines of smaller volume are intersècted (volume lines are not shown in the figure), so also are met lines of greater quality or higher values of $x$. That is, an adiabatic compression of steam near its saturation point eauses reëvaporation, the heat for which is obtained at the expense of the external work done during eompression. When the point $c$ is reached, further isentropie compression results in superheating the steam, as was first announeed by Cazin. Conversely, starting from $c$, an isentropic expansion results in a condensation or lowering of the quality $x$, as was first stated by Hirn. That is, the external work is now done by the expanding steam at the expense of the heat of vaporization of a part of it.

From the same figure it will be noticed that the $x$ lines for those qualities less than 0.5 in general slope to the left, while the slope is toward the right for those qualities greater than about 0.5. Hence for the isentropic line efg, when the mixture is near its liquid condition, it is evident that compression results in condensation, and, vice versa, expansion results in reëvaporation, as was stated in Section 44.

An isentropic line as $m n$ of Figure 32 if produced will in general cut an $x$ line in two points. Hence for each $x$ line an isentropie may be drawn tangent to it at some point, as $o$. If the line $o^{\prime} o^{\prime \prime}$ be drawn through all these possible points of tangency, its general appearance will be as shown in the figure. Such a line is ealled the "zero line" (because it represents states for which the speeifie heat may be shown to be zero). ${ }^{1}$

The ehanges in the state of a mixture undergoing an isentropic compression may then be expressed in a more rigorous form by saying that for values of $x$ greater than the zero line values there is a reëvaporation, and for values less than the zero line values there is a condensation. Conversely, for isentropic expansion there will be condensation (or reëvaporation) for values of $x$ greater than (or less than) the zero line values.

Since for an isentropic ehange the external work performed by the steam is at the expense of the intrinsic energy, the work $w$ per pound of a steam mixture undergoing a reversible adiabatic expansion from state 1 to state 2 is

$$
w=\epsilon_{1}-\epsilon_{2}=q_{1}+x_{1} \rho_{1}-q_{2}-x_{2} \rho_{2} . \quad . \quad . \quad .
$$

${ }^{1}$ Berry, "Temperature Entropy Diagram," pp. 25-26. 
53. Specific Heat of Steam. 'The specific heat of dry steam may be found for any of the following conditions: (a) superheating under constant pressure, (b) superheating at a constant volume, (c) for changes of both pressure and volume so related that the whole mass remains dry saturated steam, that is, for changes such that the temperature and entropy are related as in the dry steam line $f l$ of Figure 31, page 69. For constant pressure the value of 0.48 may be used for temperatures near that of saturation; for higher temperatures, values are given in the Table on page 93. For constant volume, Regnault gave 0.346 , but this determination is not entirely reliable.

For condition (c) the specific heat is negative for all ordinary temperatures and pressures. The physical significanee of this fact may best be shown by an example. Thus, suppose it is desired to raise the temperature of one pound of steam from $212^{\circ}$ to $213^{\circ}$ Falirenheit in accordance with condition (c) above. This may be done by compressing the steam under a pressure which increases according to the relation plotted in Figure 29 of page 64 . The speeific volume decreases with this increased temperature and pressure. The external work done may be taken as approximately equal to the product of the average pressure and the decrease in volume. This is work done upon the steam by some external source. The ehange in total heat contents represents the amount of heat which must be added from soine external source. The values of $p, q, s$, and $r$ for $212^{\circ}$ and for $213^{\circ}$ Fahrenheit are given below.

$\begin{array}{cccc}212^{\circ} & 213^{\circ} & 212^{\circ} & 213^{\circ} \\ q=180.3 & q=181.3 & p=14.70 & p=14.99 \\ r=\frac{969.7}{1150.0} & r=\frac{969.1}{1150.4} & s=26.78 & s=26.29 \\ \lambda= & & \end{array}$

Hence, 0.4 B. t. u. must be supplied from some external source to change from $212^{\circ}$ to $213^{\circ}$. But an amount of external work

$$
\frac{14.70+14.99}{2} 144(26.78-26.29)=1020 \mathrm{ft.} \mathrm{lb.}=1.35 \text { B. t. u. }
$$

has been done upon the steam. Now, to maintain the required conditions, external work to the value of $1.35 \mathrm{~B}$. t. u. has been done, but only $0.4 \mathrm{~B}$. t. $\mathrm{u}$. are needed to change the steam from $212^{\circ}$ to $213^{\circ}$. Hence, unless this excess of $(1.35-0.4)$ obtained from the external work is to go into superheating, it must be subtracted. 
This amount of heat per pound or $0.95 \mathrm{~B} . \mathrm{t}$. u. must therefore be conducted away, and hence the specific heat is negative. ${ }^{1}$

54. Résumé of Equations for a Mixture of Steam and Water. The following equations refer to a pound of mixture, and are numbered as in the preceding sections.

Total Heat Contents.

or

$$
\begin{aligned}
& \lambda=q+r=q+\rho+A p u=A \epsilon+A p u \\
& \lambda=q+x r=q+x \rho+x A p u=A \epsilon+x A p u . .
\end{aligned}
$$

Total Entropy.

$$
\phi=\phi_{w}+\phi_{v}=\int_{\theta=41.5 .5}^{\theta} \frac{c d \theta}{\theta}+\frac{r}{\theta}, \quad \text { or } \phi=\phi_{w}+x \phi_{v} . .
$$

Total Intrinsic Energy.

$$
A \epsilon=q+\rho, \text { or } A \epsilon=q+x \rho . \quad . \quad . \quad .
$$

Specific Volume. For water $\sigma=0.016$. For steam (dry) $s$.

$$
v=x s+(1-x) \sigma=x u+\sigma . \quad \text {. . . . }
$$

Increase of Volume. $u=s-\sigma$, or $x u$. . . . . . . (44)

Constant Heat Transformation.

$$
\lambda_{1}=\lambda_{2}, \quad \text { or } q_{1}+x_{1} r_{1}=q_{2}+x_{2} r_{2} \ldots . . .
$$

Constant Energy Transformation.

$$
\epsilon_{1}=\epsilon_{2}, \quad \text { or } q_{1}+x_{1} \rho_{1}=q_{2}+x_{2} \rho_{2} . \quad . \quad . \quad .
$$

$\left.\begin{array}{l}\text { Isothermal Transformation. } \\ \text { Constant Pressure. }\end{array}\right\} q, \theta$, and $p$ are constant.

$$
w=p\left(v_{2}^{\prime}-v_{1}\right)=p u\left(x_{2}-x_{1}\right) \text {. }
$$

Isentropic Transformation.

Reversible Adiabatic.

$$
\begin{gathered}
\phi_{1}=\phi_{2}=\phi_{v v_{1}}+x_{1} \phi_{v_{1}}=\phi_{v v_{2}}+x_{2} \phi_{v_{2}} . . . \\
w=\epsilon_{2}-\epsilon_{1}=\frac{1}{A}\left(q_{2}-q_{1}+x_{2} \rho_{2}-x_{1} \rho_{1}\right) . .
\end{gathered}
$$

Irreversible Adiabatic Change.

$$
d \mathfrak{q}=0, \text { but } d \phi \neq 0 \text {. }
$$

1 This method is of course unsatisfactory for the purpose of determining this specific heat because of the large errors introduced in a difference between two quantities where the difference is of a magnitude not much greater than the probable error in the quantities subtracted. For the method used by Clausius see Preston, "Heat," pp. 655-658. For 212 Fahrenheit Clausius gives 1.13. The method used above is selected because it is free from differential notation, and emphasizes the physical relations involved. 


\section{PROBLEMS AND SOLUTIONS: SATURATED WATER VAPOR}

16. Quality. How much heat has been required to change one pound of water at $60^{\circ}$ Fahrenheit into a mixture of steam and water occupying 2 cubic feet at a pressure of 130 pounds per square inch absolute?

Solution. From Table 3,

$$
\begin{aligned}
& \text { For } 130 \text { pounds } \quad \text { For } 60^{\circ} \mathrm{F} \text {. } \\
& q_{2}=318.6 \quad q_{1}=28.1 \\
& v_{2}=872.1 \\
& s_{2}=3.451
\end{aligned}
$$

By eq. (49), $v_{2}=2=x_{2} s_{2}+\left(1-x_{2}\right) \sigma$ or $x_{2}=\frac{2-0.016}{3.450-0.016}=0.577$.

By eq. (48), $\lambda_{2}-\lambda_{1}=q_{2}+x_{2} r_{2}-q_{1}=318.6+(0.577)(872.1)-28.1=793$ B.t.u.

17. Intrinsic energy. Find the intrinsic energy, volume, and entropy of a mixture of 5 pounds of steam and water which is 80 .per cent steam, if the pressure is 120 pounds absolute per square inch. How much heat must be added to make the quality unity at this pressure?

Solution. From Table 3, for 120 pounds,

$$
q=312.3, \quad r=876.9, \quad \rho=794.2, \quad \phi_{w}=0.4922, \quad \phi_{v}=1.0951, \quad s=3.723 .
$$

Given $x=0.80$.

By eq. (51), energy per pound $=778[312+(0.80)(794.2)]=736,000$ foot pounds, or $3,680,000$ foot pounds for 5 pounds.

By eq. (55), entropy per pound $=0.4922+(0.80)(1.0951)=1.3682$, or 6.841 for 5 pounds.

By eq. (48), total heat per pound $=312.3+(0.80)(876.9)=1013$ B. t. u., or 5065 B. t. u. for 5 pounds.

The total heat for $x=1.00$ is $312.3+876.9=1189.2$ B.t. u. per pound, or 5946 B. t. u. for 5 pounds.

Therefore the heat which must be added is $5946-5065=881$ B. t. u.

By eq. (49),

the volume per pound $=(0.80)(3.723)+(1-0.80)(0.016)=2.99$ cubic feet.

18. Isoenergic transformation. If three pounds of a mixture of steam and water at a pressure of 120 pounds absolute and a quality of 0.90 undergoes an isoenergic change until the pressure is 150 pounds absolute, find the quality and the volume in the final condition. 
Solution. From Table 3,

For 120 pounds

$$
\begin{aligned}
& q_{1}=312.3 \\
& \rho_{1}=794.2
\end{aligned}
$$

$$
\begin{gathered}
\text { For } 150 \text { pounds } \\
q_{2}=330.0 \\
\rho_{2}=779.3 \\
s_{2}=3.014
\end{gathered}
$$

Given $x_{1}=0.90$.

By eq. (51),

$$
q_{1}+x_{1} \rho_{1}=q_{2}+x_{2} \rho_{2} .
$$

Therefore

$$
\begin{aligned}
x_{2} & =\frac{312.3+(0.90)(794.2)-330.0}{779.3}=0.893 . \\
v_{2} & =x_{2} s_{2}+\left(1-x_{2}\right) \sigma . \\
v_{2} & =(0.893)(3.014)+(1-0.893) \sigma \text { per pound } \\
& =2.70 \text { cubic feet per pound, }
\end{aligned}
$$

By eq. (49),

or for 3 pounds the volume is 8.10 cubic feet.

19. Isothermal transformation. Three pounds of steam and water at 140 pounds absolute occupy 6 . cubic feet. If the volume is increased at constant pressure to 9 cubic feet, find the heat added, the external work done, the final quality, and the increase in entropy.

Solution. The volume per pound is 2 cubic feet in state 1 and 3 cubic feet in state 2.

From Table 3 , for 140 pounds,

By eq. (49),

$$
\rho=784.0, \quad A p u=83.4, \quad \phi_{v}=1.0675, \quad s_{1}=3.220 .
$$

Therefore

$$
\begin{aligned}
& v_{1}=x_{1} s+\left(1-x_{1}\right) \sigma . \\
& x_{1}=\frac{2-0.016}{3.220-0.016}=0.620 . \\
& x_{2}=\frac{3-0.016}{3.220-0.016}=0.932 .
\end{aligned}
$$

Similarly,

The heat added is

$$
\lambda_{2}-\lambda_{1}=x_{2} r-x_{1} r=\left(x_{2}-x_{1}\right)(\rho+A p u),
$$

$$
(0.932-0.620)(784+83.4)=245+26=271 \text { B.t. u. per pound. }
$$

Of this added heat 26 B.t. u. represents the external work. For 3 pounds the added heat is $813 \mathrm{B.t.u}$. and the external work is

$$
(3)(778)(26)=60,400 \text { foot pounds. }
$$

The increase in entropy is $\left(x_{2}-x_{1}\right) \phi_{v}=(0.932-0.620)(1.0675)=0.333$ per pound, or 1.0 unit of entropy for 3 pounds.

20. Adiabatic transformation. Five pounds of steam of quality 0.80 expand adiabatically from 130 pounds to 30 pounds absolute pressure. Find the quality, the change in volume, and the external work done.

Solution.

$$
\begin{aligned}
\text { For } 130 \text { pounds } \\
q_{1}=318.6 \\
\rho_{1}=789.0 \\
\phi_{w_{1}}=0.5000 \\
\phi_{v_{1}}=1.0808 \\
s_{1}=3.451
\end{aligned}
$$

For 30 pounds

$$
\begin{aligned}
q_{2} & =219.1 \\
\rho_{2} & =868.2 \\
s_{2} & =13.74 \\
\phi_{w_{2}} & =0.3687 \\
\phi_{v_{2}} & =1.3305 \\
s_{2} & =13.74
\end{aligned}
$$


Given $x_{1}=0.80$.

By eq. (55), for an adiabatic expansion,

Therefore

$$
\begin{aligned}
\phi_{w_{1}}+x_{1} \phi_{v_{1}}=\phi_{w_{1}}+x_{2} \phi_{v_{2}} . \\
x_{2}=\frac{0.5000+(0.80)(1.0808)-0.86887}{1.3305}=0.75 .
\end{aligned}
$$

By eq. (56), the work per pound is

or

$$
\epsilon_{1}-\epsilon_{2}=\frac{1}{A}\left(q_{1}+x_{1} \rho_{1}-q_{2}-x_{2} \rho_{2}\right),
$$

$778[318.6+(0.80)(789)-219.1-(0.75)(868.2)]=778(79.5)=62,000 \mathrm{ft} . \mathrm{lb}$. or 310,000 foot pounds for 5 pounds of mixture.

By eq. (49),

$$
\begin{aligned}
& v_{1}=(0.80)(3.450)+(1-0.80) \sigma, \\
& v_{2}=(0.75)(13.74)+(1-0.75) \sigma .
\end{aligned}
$$
and

The increase of volume per pound is (neglecting $\sigma$ ),

$$
(0.75)(13.74)-(0.80)(3.45)=7.63 \text { cubic feet, or } 38.15 \text { for } 5 \text { pounds. }
$$

21. Constant volume transformation. A closed tank containing 10 pounds of dry steam at 130 pounds absolute is cooled until 90 per cent of the steam has been condensed. What is the final temperature and how much heat has been removed?

Solution. Since the change takes place at constant volume, no external work is done, and the heat removed is that which is required to reduce the intrinsic energy from state 1 to state 2 .

$$
\begin{gathered}
\text { For } 130 \text { pounds } \\
q_{1}=318.6 \\
\rho_{1}=789.0 \\
s_{1}=3.451
\end{gathered}
$$

Given $x_{1}=1.00$ and $x_{2}=0.10$. Solving for 1 pound,

By eq. (49),

$$
v_{1}=s_{1} \text { and } v_{2}=x_{2} s_{2}+\left(1-x_{2}\right) \sigma=0.10 s_{2}+(0.90)(0.016) .
$$

For constant volume,

$$
v_{1}=v_{2},
$$

hence

$$
3.451=0.10 s_{2}+0.014, \quad \text { or } s_{2}=34.87 \text {. }
$$

From tables similar to Table $3, s_{2}=34.37$ is found to be the specific volume of steam at $198.9^{\circ}$ Fahrenheit and 11.3 pounds pressure.

For these conditions, $\quad q_{2}=167.1, \rho_{2}=905.6$.

The heat removed is $\epsilon_{1}-\epsilon_{2}$, where, by eq. (51),

$$
\epsilon_{1}=318.6+789.0 \text { and } \epsilon_{2}=167.1+(0.10)(905.6) ;
$$

that is, 850 B.t.u. per pound, or 8500 B.t.u. per 10 pounds.

22. Boiler explosion. How much energy is liberated if a boiler containing two tons of water at $353^{\circ}$ Fahrenheit explodes? Assume the atmospheric pressure to be 14.7 pounds. Draw the $\theta-\phi$ dia- 
gram. What volume would the steam thus produced occupy if there was no condensation?

Solution.

At $353^{\circ} \mathrm{F}$.

at 14.7 pounds,

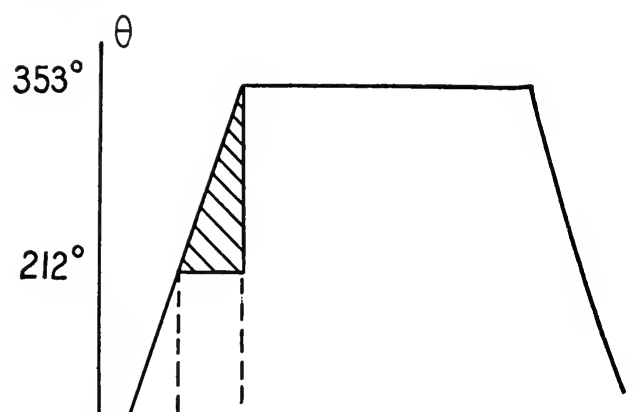$$
q_{2}=180.3 \text {. }
$$

$$
p=140 \text { pounds, } \quad q_{1}=324.4 ;
$$

Hence the heat energy liberated per pound is $q_{1}-q_{2}$, or 144 B.t.u.

For 2 tons this is 576,000 B.t.u., that is $448,000,000$ foot pounds.

The $\theta-\phi$ plot is shown to scale in Figure 33, where the heat liberated is represented by the shaded area.

If this energy is used entirely to produce reevaporation at a pressure of 14.7 pounds, the number of pounds of steam produced is found as follows. From Table $3, r$ for 14.7 pounds is 969.7 . For each pound of water there is then evaporated $x=\frac{144}{969}=0.149$ pound. The volume occupied by this steam is $0.149 \mathrm{~s}$ or $0.149(26.78)=3.98$ cubic feet. The steam produced by the explosion would fill 4000 (3.98) or 15,920 cubic feet at the atmospheric pressure. Actually the steam would in part be formed at higher pressure, there would be destructive expansion, and the values calculated above represent not actual conditions but theoretical conditions which are useful only in giving some ideas of the magnitude of the energy liberated.

23. Equivalent evaporation and boiler horse power. What is the equivalent evaporation from and at $212^{\circ}$ Fahrenheit per pound of coal in a boiler where 19,000 pounds of feed water at a temperature of $70^{\circ}$ Fahrenheit are converted into steam at 80.3 pounds gauge by the combustion of one ton of coal? 
If a boiler horse power is defined as the capacity of a boiler which evaporates 30 pounds of water per hour from a temperature of $100^{\circ}$ Fahrenheit against a pressure of 70 pounds gauge, find the equivalent evaporation from and at $212^{\circ}$ Fahrenheit, which is equal to a boiler horse power. If the above evaporation took place in two hours, what is the boiler horse power?

Solution. From Table 3,

$\begin{array}{ccccc}\text { For } 80.3+14.7 \mathrm{lb} . & \text { For } 70^{\circ} \mathrm{F} . & \text { For } 70+14.7 \mathrm{lb} . & \text { For } 100^{\circ} \mathrm{F} . & \text { For } 212^{\circ} \mathrm{F} . \\ q=294.6 & q=38.1 & q=286.4 & q=68 & q=180.3 \\ r=890.5 & & r=896.7 & & r=969.7\end{array}$

Per pound of coal, the given boiler evaporates $\frac{19,000}{2000}=9.5$ pounds of water from $70^{\circ}$ Fahrenheit to steam at 95 pounds absolute. The B.t. u. required are evidently $294.6+890.5-38.1=1147.0$ per pound.

Per pound of water from and at $212^{\circ}$ the B. t. u. required are 969.7. The equivalent evaporation of the given boiler is evidently $9.5 \frac{1147.0}{969.7}=11.25$ pounds of water
from and at $212^{\circ}$ per pound of coal.

Now a boiler horse power is equivalent to $30(286.4+896.7-68)=30(1115.0)$ B.t. u. per hour. That is, a boiler horse power is equal to an equivalent evaporation of $30 \frac{1115.0}{969.7}=34.5$ pounds per hour.

The actual boiler had an equivalent evaporation per hour of $\frac{2000}{2} 11.25=11,250$ pounds. Hence, a boiler horse power of $\frac{11,250}{34.5}=325$.

24. Surface condenser. A surface condenser receives steam at a pressure of 4 pounds absolute. Twenty pounds of water at $60^{\circ} \mathrm{Fahr}$ enheit flow through the pipes of the condenser for every pound of steam that is condensed. What is the temperature of the feed water leaving the condenser? The condenser water flows to a Worthington cooling tower, where it trickles down through tiles that cause it to spread over a large surface. If five per cent is evaporated and carried away by a current of air from a fan passing through the tower, other things being equal, what would be the final temperature of the remaining 95 per cent?

Solution. For 4 pounds pressure, $q=121.0$ and $r=10055$, or $\lambda_{1}=1126.5$ B.t. u. For $60^{\circ}$ Fahrenheit temperature, $q=28.1=\lambda_{2}$.

Let $q_{x}$ represent the heat of the liquid per pound for the final common temperature of the steam and condenser water.

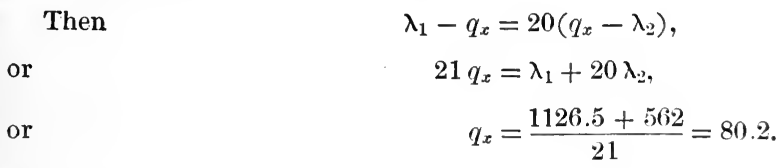

Hence the temperature is $112^{\circ}$ Fahrenheit. 
To evaporate 5 per cent of a pound of water at $112^{\circ}$ Fahrenheit requires $0.05 r$ B. t. u. For $112^{\circ}$ Fahrenheit, $r$ is 1029 . Hence it requires 51.5 B.t. u.

The abstraction of $51.5 \mathrm{~B}$. t. u. from the remaining 95 per cent of the pound to reevaporate the 5 per cent reduces the heat of the liquid for the water to $[(0.95)(80.2)$ -51.5] B.t.u. for each 95 per cent of a pound. Hence per pound of remaining water $q$ is $\frac{(0.95)(80.2)-51.5}{0.95}=26$ B. t.u. The corresponding temperature is $26+32$, or $58^{\circ}$ Fahrenheit. (Actually the temperature of the cooling water and the condensed steam are not exactly the same.)

25. Jet condenser. A jet condenser, which injects coolirg water into the steam which is to be condensed, receives steam at 4 pounds absolute. The water of the jet is $60^{\circ}$ Fahrenheit. If the feed is $120^{\circ}$ Fahrenheit, how much condensing water is used per pound of steam condensed?

Solution. Using the values of $\lambda$ from Problem 24 and $q$ for $120^{\circ}$ Fahrenheit as $88 \mathrm{~B}$.t. u., if we represent by $m$ the mass of the condensing water used per pound of steam condensed, then

or

$$
\begin{aligned}
(1126.5-88) & =m(88-28.1), \\
m & =\frac{1126.5-88}{88-28.1}=17.3 \text { pounds. }
\end{aligned}
$$

26. Rankine cycle. The operations of a steam engine are, essentially, the admission of steam, its expansion, and its final expulsion. The quantity of the working fluid in the engine is not always the same, and for this reason the $p$-v plot of the engine does not represent a closed cycle in the sense in which the cycle of the Carnot engine of page 22 is a closed cycle. Considering the boiler, the engine, and the condenser as a unit, the steam does pass through a closed cycle. The assumptions of the theoretical and ideal Rankine cycle are as follows: (1) Water is raised from the temperature at which it is fed into the boiler to the temperature and pressure at which it is to be admitted to the engine cylinder. (2) When the admission valve of the engine opens, an isothermal expansion occurs in the boiler and engine cylinder while the piston is moved forward by this expansion until cut-off occur's. (3) The steam then expands adiabatically in the engine cylinder until its pressure is that of the condenser. (4) It is then expelled by the back stroke of the piston and condensed at the constant temperature and pressure of the condenser. This condensed water is then fed back into the boiler.

Draw the $p-v$ and $\theta-\phi$ plots of such a Rankine cycle for one pound of water received by the engine and expelled at a condenser pressure such that the vacuum gauge reads 24.4 inches of mercury. The boiler pressure is 80 pounds gauge, the atmospheric pressure is 15 pounds. Calculate the efficiency. 
Solution. The $p-v$ plot is shown in Figure 35 , which is drawn to scale to emphasize the volume relations. The $\theta-\phi$ plot is also drawn to scale and is shown in Figure 84 . The work done may most easily be found from the $\theta-\phi$ plot. The shaded area abrel represents the work done in heat units. The efficiency is evidently

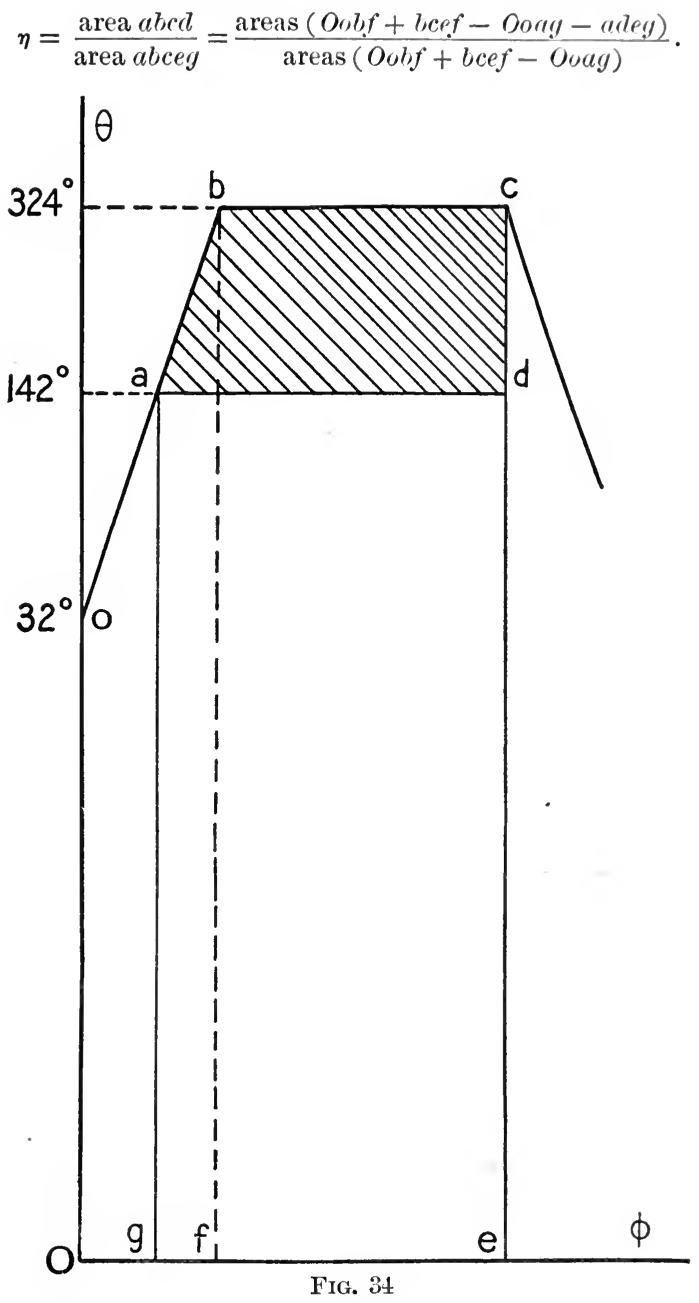

Replacing these areas by their heat equivalents, we have

$$
\eta=\frac{q_{1}+r_{1}-q_{2}-x_{2} r_{2}}{q_{1}+r_{1}-q_{2}}=1-\frac{x_{2} r_{2}}{q_{1}+r_{1}-q_{2}} \cdot
$$

The boiler pressure is $80+15=95$ pounds absolute. Since 30 inches of mercury correspond to a pressure of $\mathbf{1 4 . 7}$ pounds, 24.t inches correspond to a condenser vacuum of $\frac{14.7}{30} 24.4=12$ pounds. Hence the condenser pressure is 3 pounds absolute. 


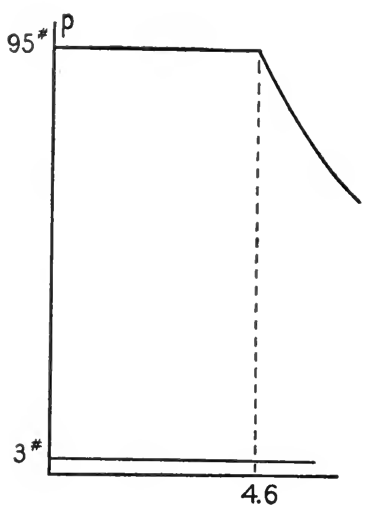

From Table 3,

For 95 pounds pressure

$$
\begin{aligned}
q_{1} & =294.6 \\
r_{1} & =890.5 \\
\phi_{w} & =0.4699 \\
\phi_{v} & =1.1363 \\
s_{1} & =4.644
\end{aligned}
$$

For 3 pounds pressure

$$
\begin{aligned}
q_{2} & =109.6 \\
r_{2} & =1012.2 \\
\phi_{w} & =0.2011 \\
\phi_{v_{2}} & =1.6841 \\
s_{2} & =118.4
\end{aligned}
$$

For the problem under solution the value of $x_{2}$ must be found by using equation (55), the condition for an isentropic expansion. Thus

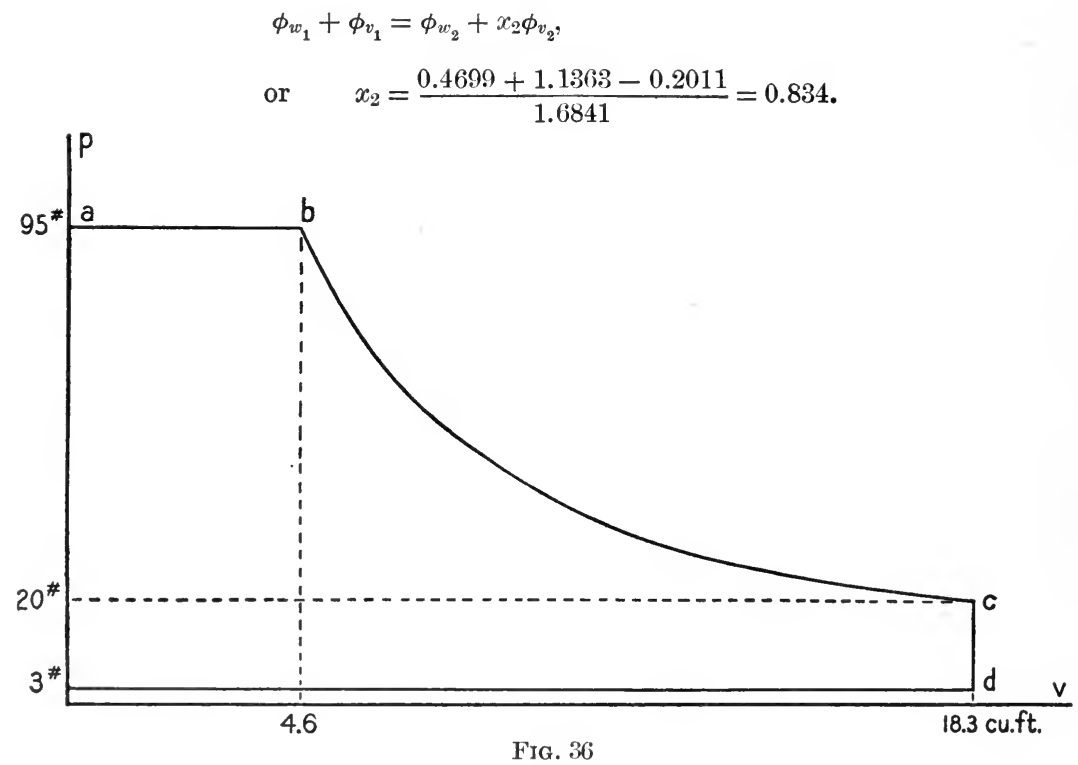

Then substitution in the equation above for the "Rankine efficiency" gives

$$
\eta=1-\frac{(0.834)(1012.2)}{294.6+890.5-109.6}=1-0.786, \text { or } 21.4 \text { per cent. }
$$

27. Incomplete expansion. Because of the large piston displacement required for a complete expansion down to the condenser pressure, the resulting small force exerted upon the piston and the 
larger amount of friction, better mechanical efficiency can be obtained by sacrificing some theoretical thermal efficiency and stopping the expansion at some pressure higher than the condenser back pressure. Such a cycle may be represented as in Figure 36 . From $c$ to $d$ the steam in an actual engine flows through the exhaust valve until the pressure has fallen to the condenser back pressure. From $d$ to $e$ the remainder of the steam is expelled by the back stroke of the engine. For all thermodynamic purposes in this discussion the effect is the same as if the steam were cooled at constant volume from $c$ to $d$, and compressed isothermally from $d$ to $e$.

If the initial pressure is 95 pounds and the condenser back pressure 3 pounds absolute as in Problem 26, find the thermal efficiency if release occurs at 20 pressure absolute. Draw the $\theta-\phi$ plot. What proportion of the total piston displacement is the cut-off in this case?

Solution. The $p-v$ and $\theta-\phi$ plots are

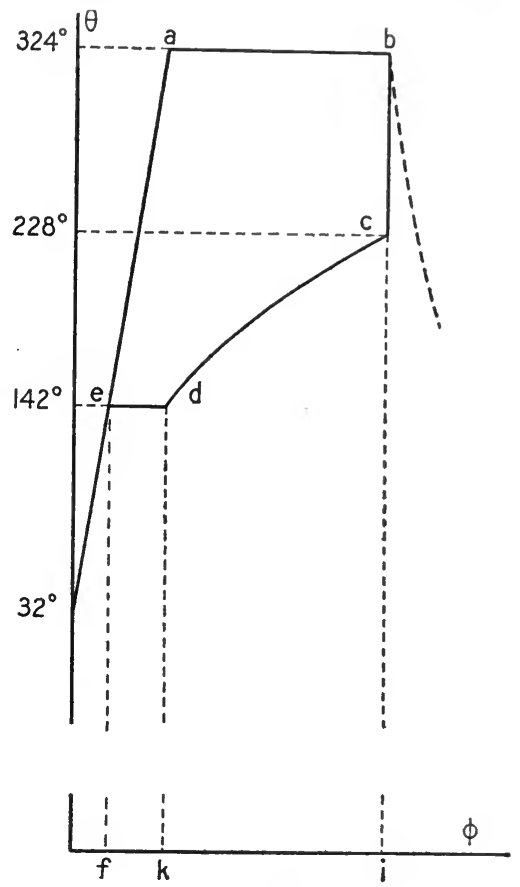

FIG. 37 shown to scale in Figure 36 and Figure 37. The efficiency may be found from the $\theta-\phi$ plot by the use of a planimeter. The line $c d$ represents the constant volume of $v_{c}$. The efficiency may also be obtained by calculations based on the $p-v$ plot as follows. Denote by 1 the state for 95 pounds pressure, by 2 that for 20 pounds, and by 3 that for 3 pounds.

Then

$$
\eta=\frac{\operatorname{areas}(a b m o+b c n m-d n o e)}{J\left(q_{1}+r_{1}-q_{3}\right)},
$$

where

$$
\text { area } a b m o=p_{a} v_{a}=p_{1} s_{1} \text { per pound of entering steam, }
$$

From Table 3 ,

$$
\begin{aligned}
\text { area } b c n m=J\left(q_{b}+\rho_{b}-q_{c}-x_{c} \rho_{c}\right)=J\left(q_{1}+\rho_{1}-q_{2}-x_{2} \rho_{2}\right), \\
\text { area } d n o e=p_{d} v_{d}=p_{3} v_{c}=p_{3}\left(x_{2} u_{2}+\sigma\right) .
\end{aligned}
$$

$$
\text { For } \begin{aligned}
p_{1} & =95 \\
q_{1} & =294.6 \\
r_{1} & =890.5 \\
s_{1} & =4.644 \\
\phi_{w_{1}} & =0.4699 \\
\phi_{v_{1}} & =1.1363 \\
\rho_{1} & =808.8
\end{aligned}
$$

$$
\text { For } \begin{aligned}
p_{2} & =20 \\
q_{2} & =196.4 \\
r_{2} & =959.4 \\
s_{2} & =20.09 \\
u_{2} & =20.07 \\
\phi_{w_{3}} & =0.3362 \\
\phi_{v_{2}} & =1.3957 \\
\rho_{2} & =885.1
\end{aligned}
$$

For $p_{3}=3$

$q_{3}=109.6$ 
For an adiabatic expansion to a pressure of 20 pounds and quality $x_{2}$ we have

$$
\phi_{w_{1}}+\phi_{v_{1}}=\phi_{w_{2}}+x_{2} \phi_{v_{2}}
$$

or

$$
x_{2}=\frac{0.4699+1.1363-0.3362}{1.3957}=0.909 \text {. }
$$

Hence from the relations established from Figure 36

Work during admission is $\quad(95)(144)(4.644)=63,200 \mathrm{ft}$. lb. per lb. of steam,

Work during expansion is by eq. (36)

$$
778[294.6+808.8-196.4-(0.909)(885.1)]=79,400 \mathrm{ft} .1 \mathrm{~b} .
$$

The combined work of admission and expansion is $\overline{142,600 \mathrm{ft} \text {. } 1 \mathrm{~b} \text {. }}$

Work during expulsion is $(3)(144)[0.909(20.07)]=7800 \mathrm{ft} . \mathrm{lb}$.

Therefore external work done by the engine is $\overline{134,800 \mathrm{ft} . \mathrm{lb}}$. per lb. of steam.

The input is $778(294.6+890.5-109.6)=\quad 832,000 \mathrm{ft}$. lb. per lb. of steam.

Hence the efficiency is $\frac{1348}{8320}=16.1$ per cent.

The volume at release per pound is $v_{c}=(0.909)(20.07)=18.3$ cubic feet.

The volume at cut-off per pound is $v_{b}=4.64$.

The cut-off is then $\frac{4.6}{18.3}=25$ per cent.

28. Efficiency and boiler pressure. Assuming a non-conducting engine working non-expansively and non-conducting, draw the $p$ - $v$ and $\theta-\phi$ plot for a boiler pressure of 90 pounds absolute and an atmospheric pressure of 14.7 pounds. The feed water is $60^{\circ}$ Fahrenheit. Calculate the efficiency for pressures of $50,70,90,110$, and 130 pounds absolute and plot a curve showing the relation between boiler pressure efficiency for this case.

Solution. The efficiency for a pressure of 90 pounds is calculated as an illustration. The $p-v$ and $\theta-\phi$ plots are shown in Figures 38 and 39
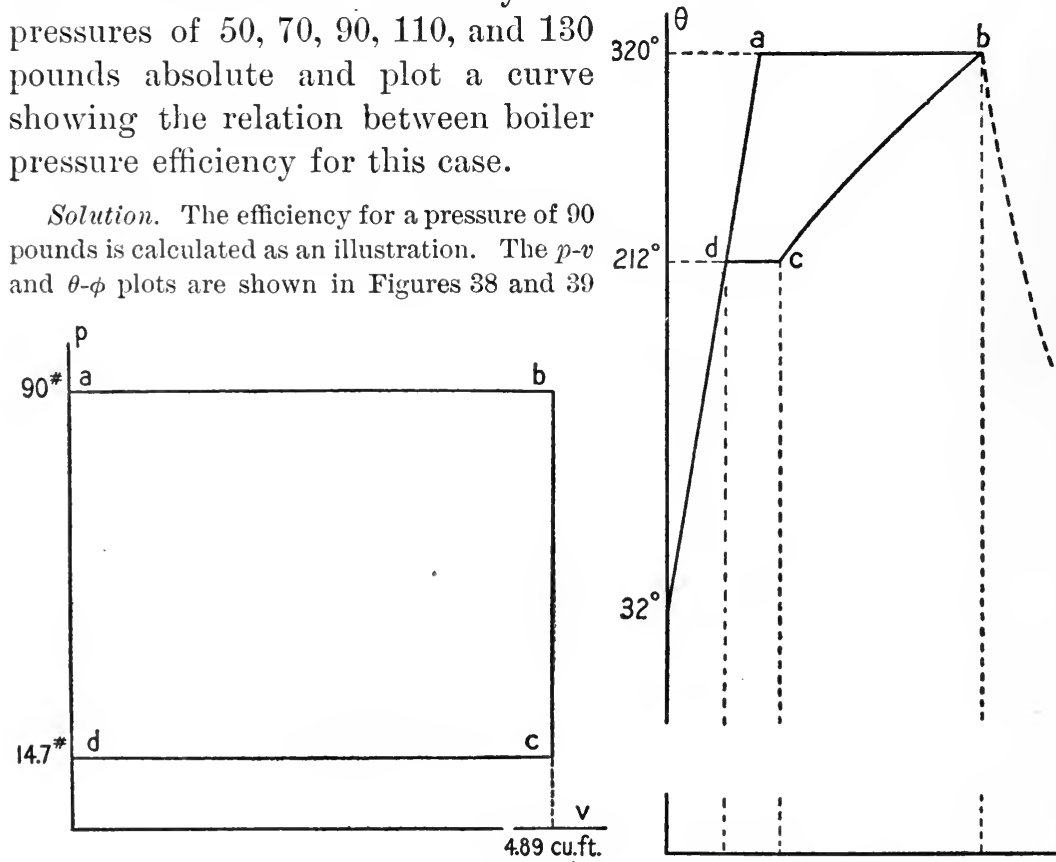

FIG. 38

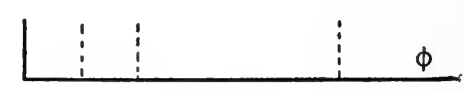

FIG. 39 
respectively. Table 3 gives for 90 pounds $q_{1}=290.7, r_{1}=893.5$, and $s_{1}=4.886$. For $60^{\circ}$ Fahrenheit $q_{3}=28.1$.

The input is $q_{1}+r_{1}-q_{3}=290.7+893.5-28.1=1156.1$ B.t.u.

The output is most conveniently found from the $p-v$ plot. It is $\left(p_{a}-p_{d}\right) v_{b}=\left(p_{1}-p_{2}\right) s_{1}$.

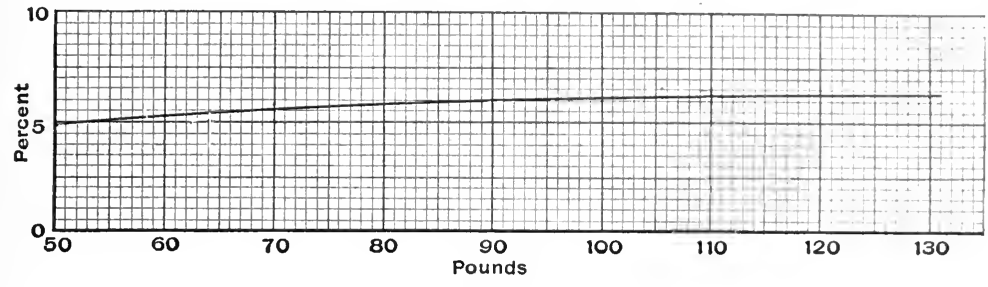

FIG. 40

Or substituting the indicated values, the output is

$(144)(90-14.7) 4.886=52,900$ foot pounds $=68$ B.t.u.

The efficiency is then $\frac{68}{1156}=5.9$ per cent.

The curve of efficiency and boiler pressure is shown in Figure 40.

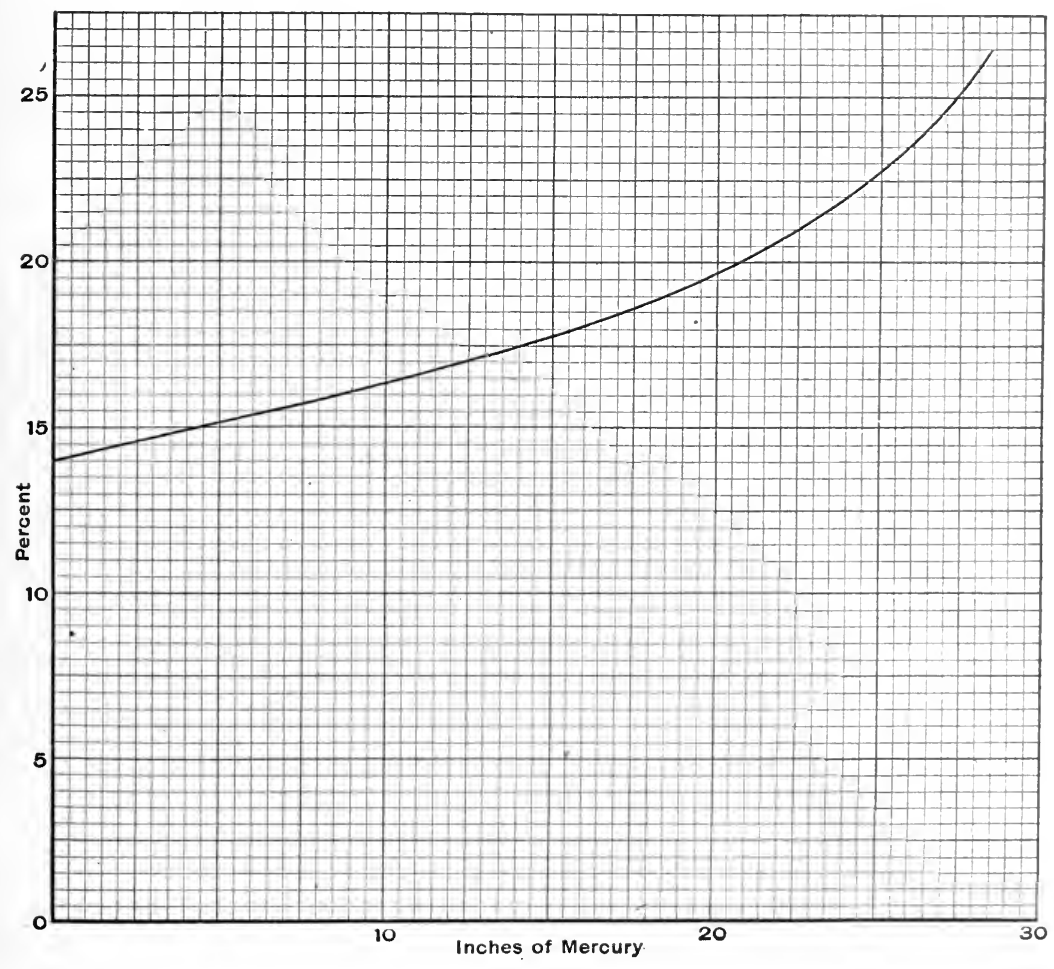

Frg. 41 
29. Condensing engine. Find for the Rankine cycle the theoretical efficiency of an engine supplied with steam at 100 pounds absolute for the following values of the condenser back pressure: 1, 2, 3, 4, 6, 10, and 14.7 pounds absolute. Plot efficiency against inches of mercury indicated by a vacuum gauge from an atmospheric pressure of 14.7 pounds.

Solution. The solution of this problem is identical with that of Problem 26. The plot of Figure 41 shows the variation of the efficiency with the condenser vacuum.

30. Use of the temperature entropy chart. Assume a frictionless non-conducting engine working on a cycle of incomplete expansion as in Problem 27. If the clearance is negligible, the cut-off 40 per cent of the piston displacement of 5 cubic feet, the admission steam at 100 pounds absolute, and the condenser back pressure 3 pounds absolute, draw the $p-v$ and $\theta-\phi$ plots.

Solution. One pound of steam at 100 pounds pressure has a volume of 4.432 cubic feet. Therefore at cut-off the engine cylinder contains $\frac{40}{100} \frac{5.00}{4.432}=0.451$ pounds.

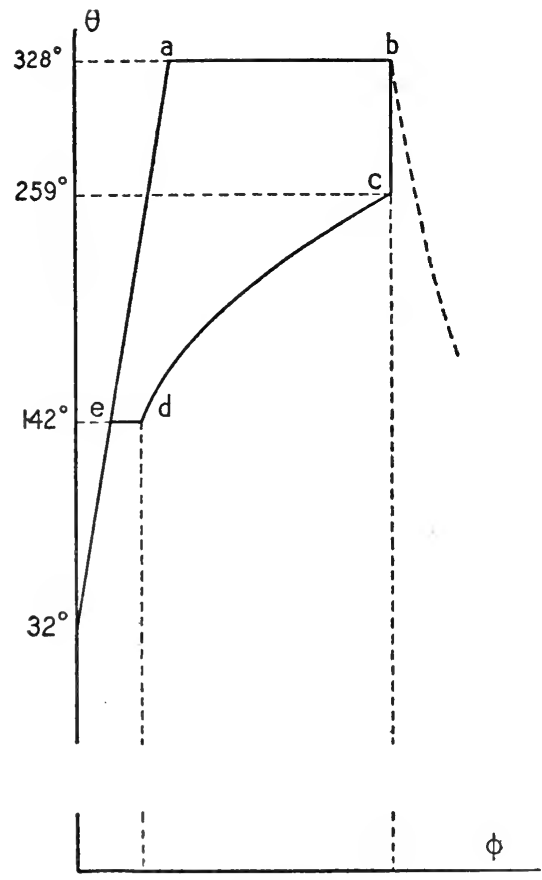

FIG. 43

Since the $\theta-\phi$ chart and the tables for steam given on page 130 are both per pound of steam, it is most convenient to reduce the engine under consideration to one containing a pound of steam (or mixture if the steam is wet). Thus, if the cylinder volume is imagined to be increased in the ratio $\frac{1}{0.451}$, that is, to $\frac{5.00}{0.451}=11.1$ cubic feet, there will be one pound of steam in the cylinder at cut-off.

From the $\theta-\phi$ chart of Figure 42 it is found that the entropy of one pound of dry steam at 100 pounds is 1.602 . Where the line of constant entropy of this value intersects the line of constant volume for 11 cubic feet gives the point $c$ on the $\theta-\phi$ plot of Figure 43, corresponding to the point $c$, which may now be plotted on the $p$ - $v$ plot of Figure 44 . If intervening points on the $p-v$ plot of the expansion are desired, they may be found by noting the intersections of the line of constant entropy, equal to 1.602 , with various lines of constant volume and then finding the pressures corresponding to those intersections. The constant volume line of value 11 cubic feet is followed until it intersects the constant pressure line for 3 pounds. This gives the point $d$ on the $\theta-\phi$ plot. 


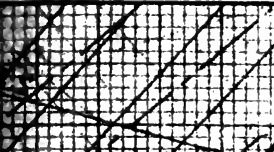

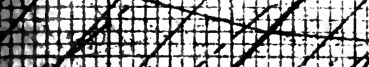

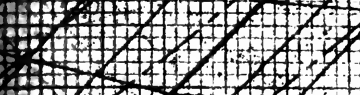

4272

$62+170107$

$1+1+101$

-1.12

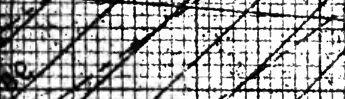

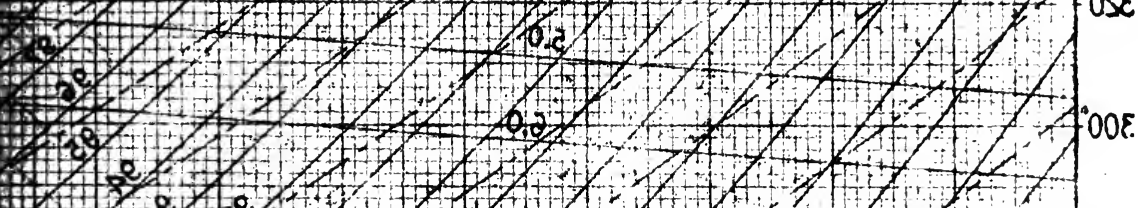

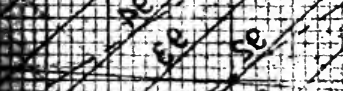

2. 1.76

$+2,120$

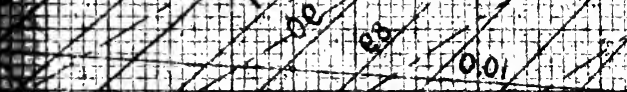

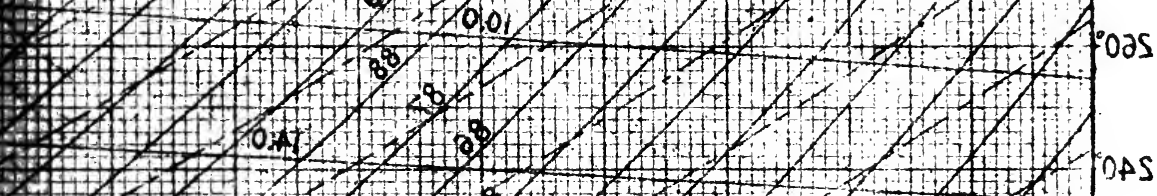

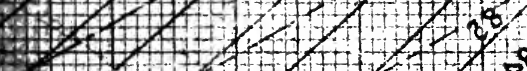

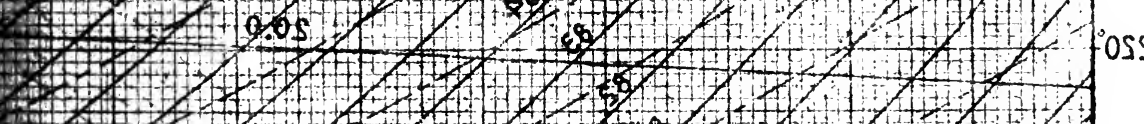

8

8.

ativis

17.17\%

160

1+2112

$4+1$

$1+1$

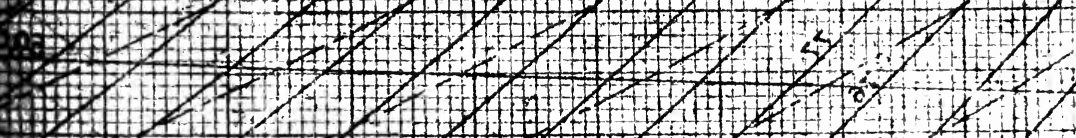

\section{臨}

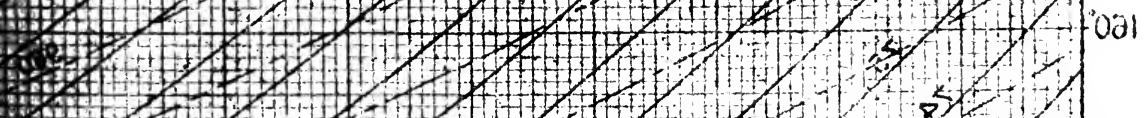

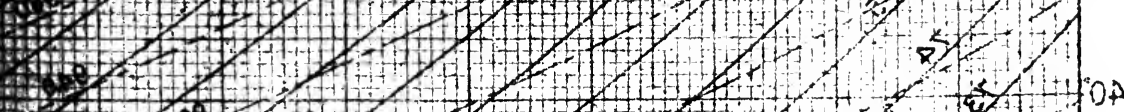
47,005

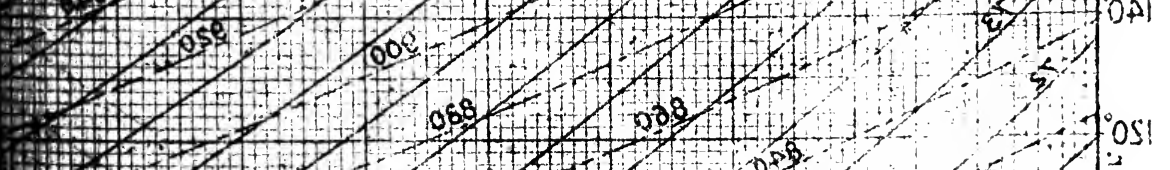

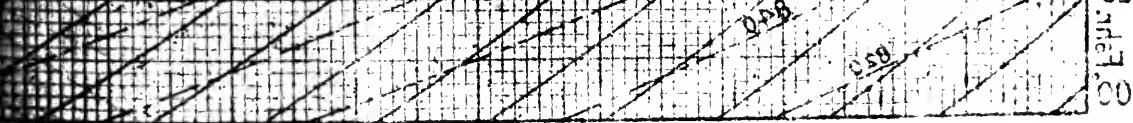

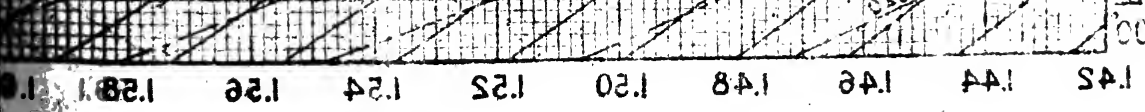




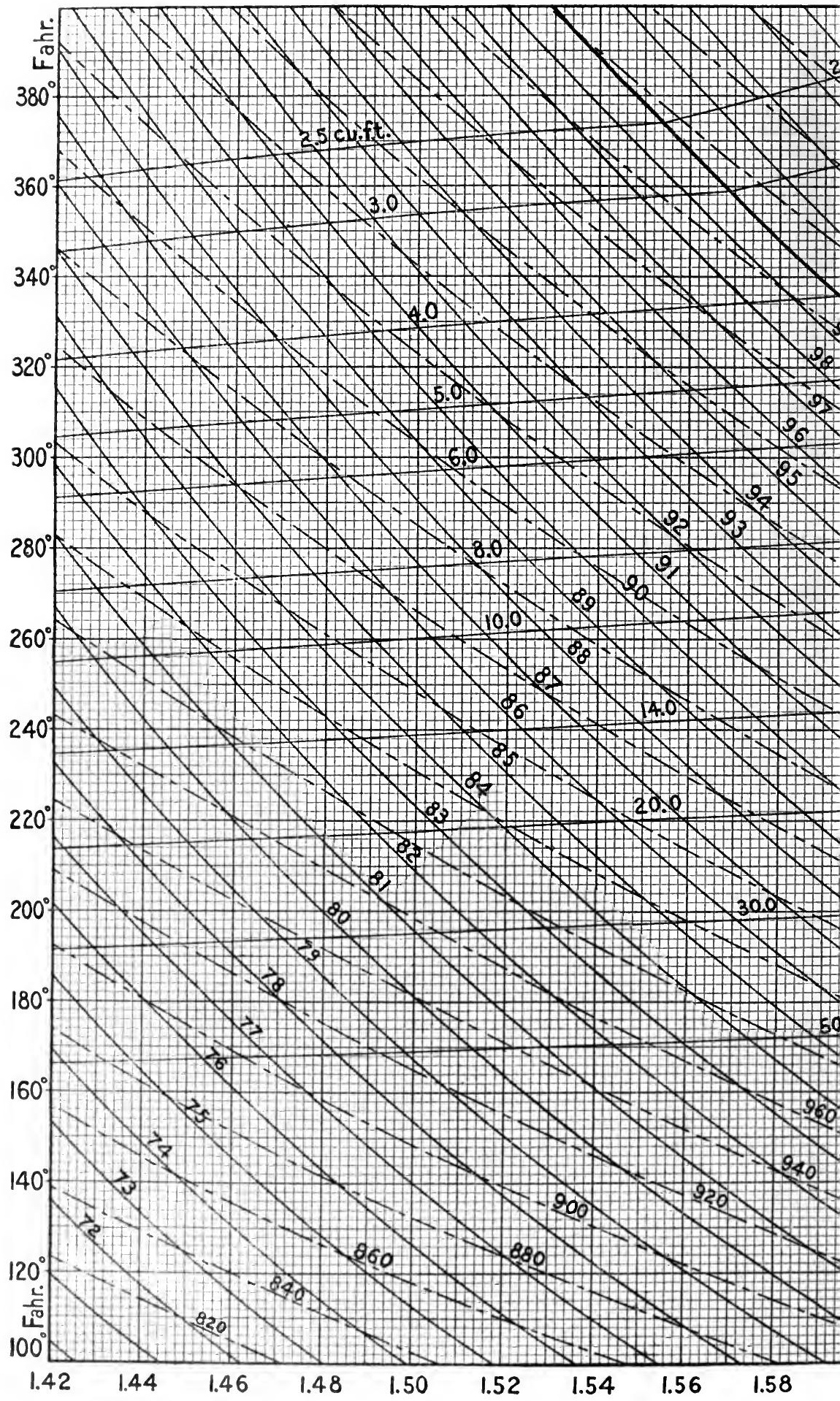




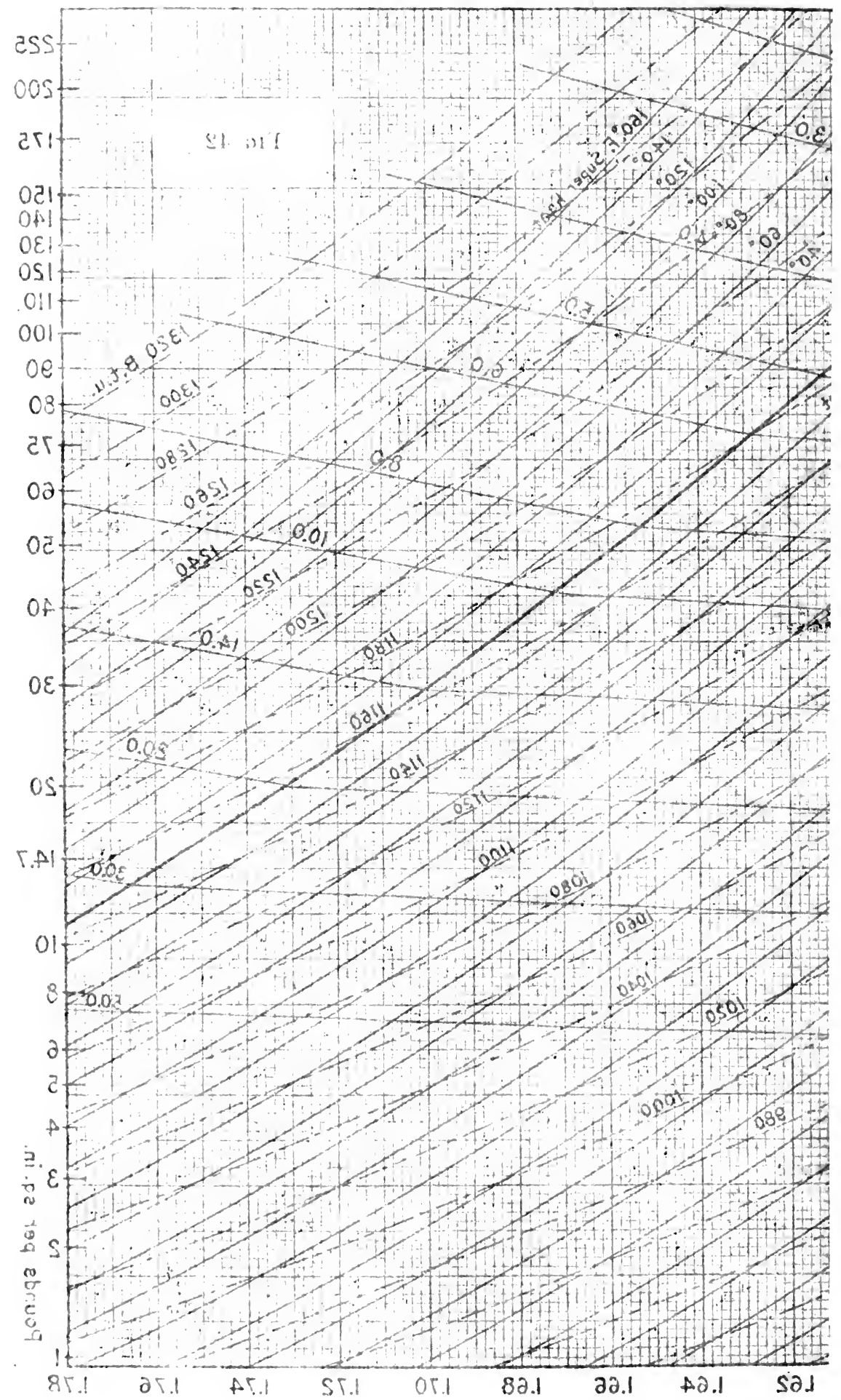


The efficiency may now be found after the manner of Problem 27. The pressure volume plot for this engine with its piston displacement of 5 cubic feet is shown dotted in Figure 44.

31. Boulvin diagram. A simple method of transforming graphically a steam cycle expressed in terms of any one of the following sets of coordinates into terms of any other set is known from its proposer as the Boulvin diagram. The coördinates are $p$ and $v, p$ and $\theta, \theta$ and $\phi$, and $\phi$ and $v$. If

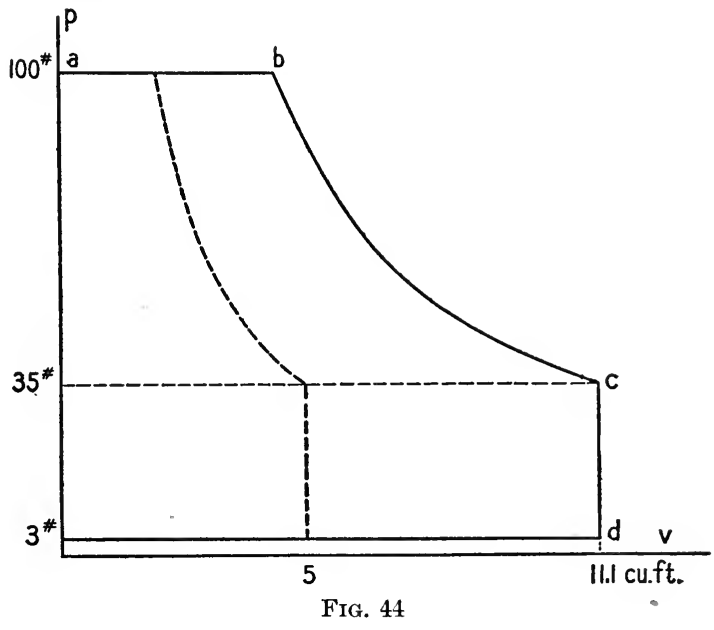
four quadrants are drawn, as in Figure 45, the method may be shown by changing a point $k_{\mathrm{iii}}$ expressed in terms of $p$ and $v$ into each of the

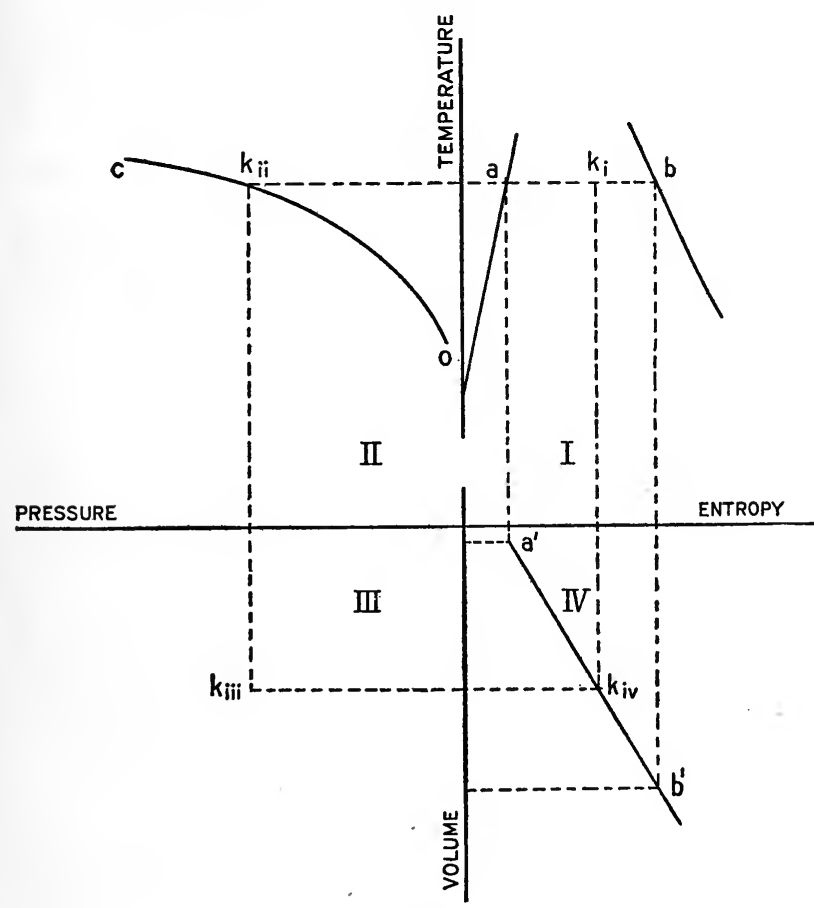

FIG. 45 other sets of coördinates. Thus, in quadrant II, draw the curve oc for the pressure-temperature relations for the boiling point of water. To any point $k_{\mathrm{iii}}$ in the pressure-volume quadrant there is possible but one temperature, namely, that corresponding to its pressure or to the temperature of $k_{\text {ii }}$ on the temperature-pressure curve of quadrant II. 
Now draw in quadrant I the $\theta-\phi$ curve $a b$ for this temperature. The line of constant temperature $a^{\prime} b^{\prime}$ in quadrant IV may then be drawn as described in Section 51. The projection of $k_{\text {iii }}$ on the curve $a^{\prime} b^{\prime}$ at $k_{\mathrm{iv}}$ then gives the entropy corresponding to the pressure (that is, to the temperature) and volume of the point $k_{\mathrm{iii}}$. The point $k_{\mathrm{i}}$ on the $\theta-\phi$ plot is then found by projecting $k_{\text {iv }}$ on $a b$.

Figure 46 shows the indicator card of an actual engine. The piston displacement was 1.037 cubic feet. Compression occurred at 3.8 per cent stroke. The amount of steam supplied per stroke to the end of the cylinder for which this card was obtained was 0.0781

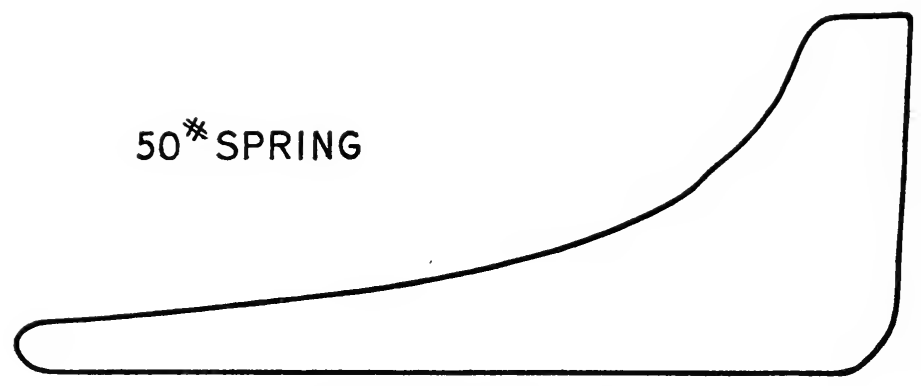

FIG. 46

pound. The clearance was 9.4 per cent of the piston displacement. The receiver back pressure was 14.7 pounds. Draw the $\theta$ - $\phi$ plot for the expansion and discuss the heat transfer indicated.

Solution. Assume the steam dry at compression. This is the ordinary assumption of steam engineering practice. All the water in the cylinder at release is supposed to reëvaporate luring the back stroke of the piston and be expelled, except for that steam which remains in the clearance spaces. ${ }^{1}$ The steam at compression then occupies $(9.4+3.8)$ per cent of 1.037 cubic foot at a pressure of $\mathbf{1 4 . 7}$ pounds. For this pressure the specific weight or density is found from 'Table 3 to be 0.0374 . Hence the clearance steam was $\frac{182}{1000}(1.037)(0.0374)=0.0052$ pound. At cut-off there was then present in the cylinder $0.0781+0.0052=0.0833$ pound of steam.

Since the $\theta-\phi$ diagram is most conveniently plotted for unit weight of steam, we

1 For further discnssion of the quality at compression see Reeve, "Thermodynamics of Heat Engines," p. 214 (Macmillan). 


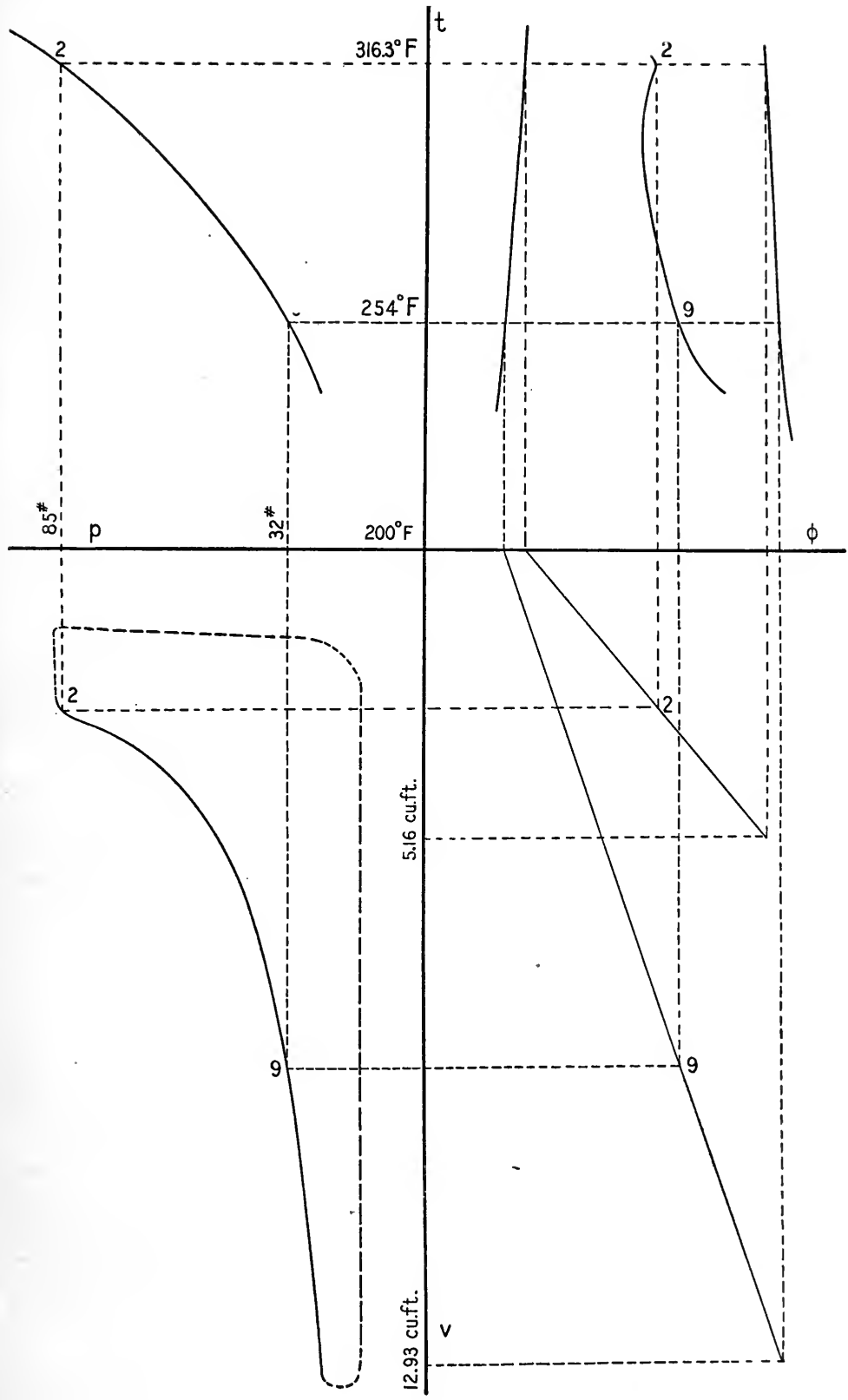

FIG. 47 
follow the method of Problem 30 and plot in the $p-v$ quadrant of the Boulvin diagram the changes in the pressure and volume per pound of mixture. That is, all the volumes of the actual problem are increased in the ratio of 1 to 0.0833 . The $p-v$ plot of quadrant III of Figure 47 shows the expansion line of the indicator card of Figure 46 so modified and plotted. The piston displacement has been increased to $\frac{1.037}{0.0833}=12.45$ cubic feet. Similarly, the clearance has become 9.4 per cent of 12.45 , or 1.17 cubic feet。

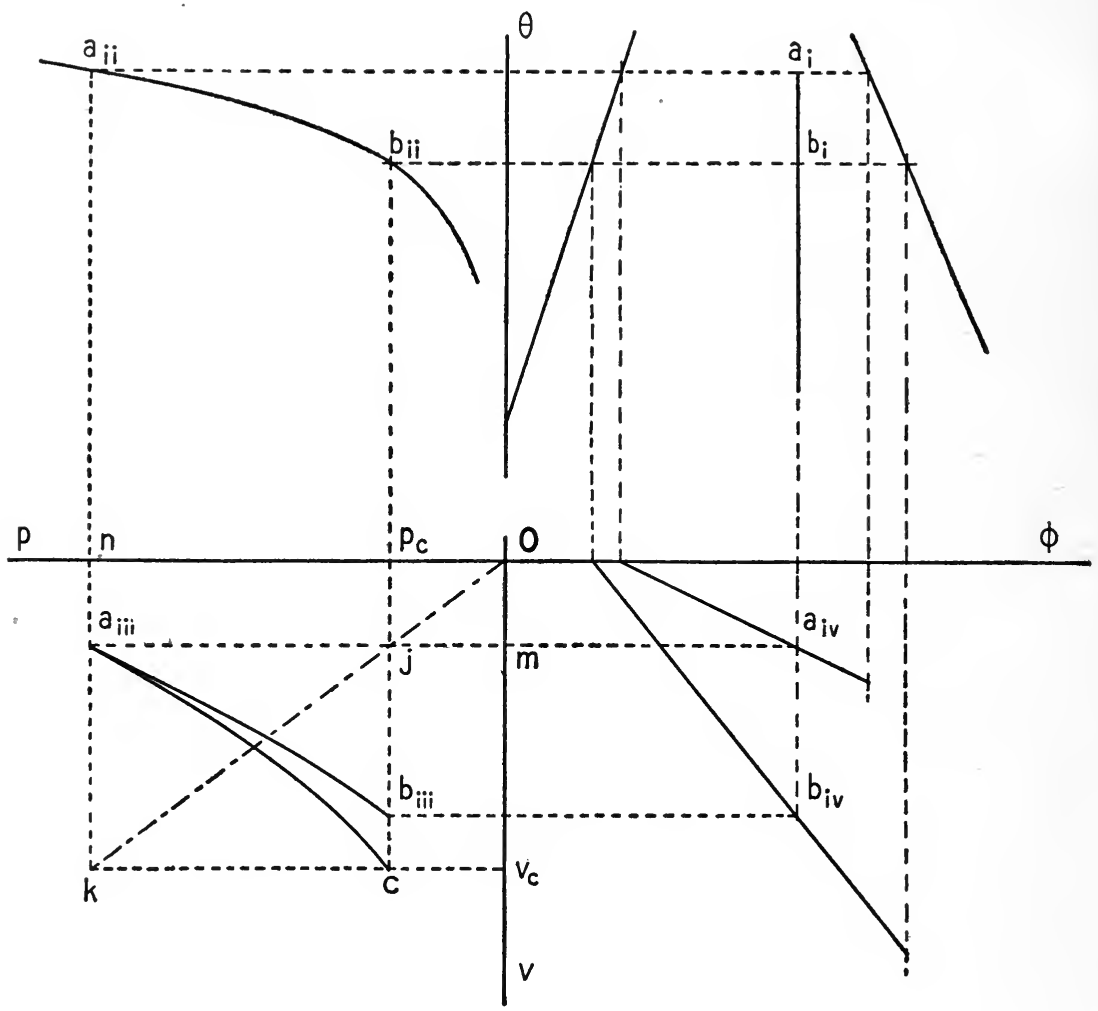

FIG. 48

The $\theta-\phi$ plot of the expansion has been constructed by the method outlined above. The construction lines for two points which have been reproduced in the plate indicate sufficiently the method.

Discussion. The steam supplied to this engine was found by means of a throttling calorimeter to have a quality of $\mathbf{9 9}$ per cent when it entered the cylinder. Due to condensation the quality had fallen to 47 per cent at cut-off. Some further condensation took place during the earlier part of the expansion. For the latter and greater part of the expansion, however, heat was returned to the steam from the cylinder walls. ${ }^{1}$ The quality therefore rose to 72 per cent at release.

1 That this heat is at a lower availability as a result of the lower temperature is evident from Problem 50. The present problem serves as an introduction to the discussion of the gain due to superheating which is made in Section 62 of page 98 . 
32. Hyperbolic expansion line. Because the actual adiabatic line for a steam transformation is not to be drawn by any simple graphical method, it is the custom of steam engineers to judge of the actual operation of a given engine by drawing through the point of cut-off on the $p-v$ plot (indicator card) of the engine a hyperbola. It is the object of this problem to compare the hyperbolic line with the true isentropic. By using the Boulvin diagram find the $p$ - $v$ plot of an isentropic line which has been assumed in the $\theta$ - $\phi$ quadrant. Then through the point of this adiabatic in the $p$-v quadrant which corresponds to the highest assumed pressure draw a rectangular hyperbola.

Solution. The construction of the adiabatic $a b$ is evident from Figure 48 . To draw the hyperbola proceed as follows. Draw $a_{i i i} m$ perpendicular to $O v$ and $a_{i i i} n$ perpendicular to $O p$. Through some point $v_{c}$ on $O v$ draw $v_{c} k$ perpendicular to $O v$. At $k$ the intersection of $v_{c} k$ and $a_{i i i} n$ draw $k O$ to the origin. Through $j$, the intersection of $k O$ and $a_{i i i} m$, draw $p_{c} j$ perpendicular to $O p$ to meet $v_{c} k$ in $c$, which is a second point on the hyperbola. In a similar manner other points may be obtained. 


\section{CHAPTER IV \\ SUPERHEATED STEAM}

55. Three Molecular States. It is a well-known fact of physics that there are three molecular conditions in which substances may exist. These are commonly stated as solid, liquid, and aeriform. The aeriform bodies are commonly subdivided into two classes; namely, those which obey Boyle's Law at ordinary temperatures and pressures and are called gases, and those which at ordinary pressures and temperatures can exist in both a liquid and an aeriform state and are called vapors. The distinction between these molecular conditions is not one depending upon the substance considered, but is one dependent wholly upon its physical conditions of pressure and temperature.

Thus it is known that the so-called "permanent gases" like hydrogen and oxygen have been liquefied and even solidified at extremely low temperatures and high pressures. For any gas there is a certain critical temperature above which it eannot be liquefied. And at this critical temperature there is a certain minimum value of the pressure which must be applied to produce liquefaction. In the development of this portion of physics the term "permanent gases" was introduced and applied to those substances in an aeriform condition which resisted the efforts that had been made to liquefy them. The term to-day, since the work of Wroblewski and Olszewski, Pictet, Cailletet, Dewar, and Linde, is evidently a misnomer.

The same substanice can exist in any one of the three principal molecular states. The commonest example of this is, of course, water, which at ordinary temperatures may coexist in all three states. For the ordinary temperatures of our atmosphere, however, the aeriform state which water assumes must be classed as a vapor state. It seems reasonable to consider that at higher temperatures it would more nearly approximate a true gaseous state.

56. Superheated Steam. Water vapor, as has already been noted in previous discussions, obeys the ordinary vapor laws by having the temperature and pressure of the liquid from which it is produced and with which it is in contact. But when removed from contact with 
its liquid (whether actually or essentially as described in Section 44), its temperature may be raised above its former value. The vapor or steam is then said to be superheated. According to that portion of the molecular theory stated above it is to be expected, then, that the pressure, specific volume, and temperature of superheated steam would tend to follow an equation somewhat of the form of the characteristic equation of a perfect gas.

In accordance with this supposition two very satisfactory formulas have been proposed. They are both of the form $p v=R \theta$, but contain corrective terms. The simplest, and for ordinary purposes one of sufficient agreement with the actual experimental results, is that suggested by Tumlirz and based upon the experiments of Battelli. It will usually be referred to as the "equation of Tumlirz." Expressed in the units of the F.P.S. system it is

$$
p v=85.85 \theta-0.256 p
$$

where $\theta$ is the absolute temperature in Fahrenheit degrees and $p$ and $v$ are specific pressure and volume.

The equation proposed by Knoblausch, Linde, and Klebe ${ }^{1}$ is more satisfactory in its agreement, but the accuracy is attained at the expense of a tedious calculation. It is expressed in the same symbols as equation (57) as follows:

$$
p v=85.85 \theta-p(1+0.00000976 p)\left(\frac{150,300,000}{\theta^{3}}-0.0833\right) .
$$

57. Specific Heat of Superheated Steam. The specific heat of superheated steam has been determined for constant pressure conditions by Knoblausch and Jakob. The following table of their values is only partial : ${ }^{2}$

\begin{tabular}{|c|c|c|c|c|c|}
\hline Pressure \# per sq. in. & $p=85.3$ & $p=113.8$ & $p=142.2$ & $p=170.6$ & $p=199.1$ \\
\hline Temperature $^{\circ} \mathrm{F}$. & $t_{0}=316$ & $t_{0}=336$ & $t_{0}=354$ & $t_{0}=368$ & $t_{0}=381$ \\
\hline$t_{1}=392$ & 0.530 & 0.560 & 0.597 & 0.635 & 0.677 \\
\hline$t_{1}=482$ & 0.514 & 0.532 & 0.552 & 0.570 & 0.588 \\
\hline$t_{1}=572$ & 0.505 & 0.517 & 0.530 & 0.541 & 0.550 \\
\hline
\end{tabular}

The table gives the average value of the specific heat (for the pressure indicated in the upper row) which should be used in calculating

1 These equations expressed in F.P.S. units are taken from the "Steam and Entropy Tables " of Professor Peabody.

2 A complete table in F. P. S. units is given in Professor Peabody's "Tables." 
the heat required to superheat from the saturation temperature (corresponding to this pressure and given in the second row) to the temperature given in the left-hand column. Illustration of the use of this table will be given in Problem 35.

For all engineering purposes steam is superheated at constant pressure. The additional heat required to raise one pound of dry steam from the condition of saturated vapor at a pressure $p_{0}$ and a temperature $t_{0}$ (or $\theta_{0}$ absolute) to a temperature $t$ (or $\theta$ ) will be denoted by the symbol $h$. That is,

$$
h=c_{p}\left(\theta-\theta_{0}\right)=c_{p}\left(t-t_{0}\right), . \quad . \quad . \quad .
$$

where $c_{p}$ represents the specific heat at constant pressure as found from the above table. The total heat contents of a pound of superheated steam at a temperature $t$ is then

$$
\lambda=q_{0}+r_{0}+h, \quad \text {. . . . . . }
$$

where $r_{0}$ and $q_{0}$ are the values for the saturation temperature $t_{0}$ which corresponds to the pressure $p$ (equal to $p_{0}$, since the superheating is at constant pressure).

For many engineering purposes the value of $c_{p}$ may be taken as 0.48 with sufficient accuracy.

58. Entropy of Superheated Steam. The increase in entropy due to superheating, expressed by the symbol $\phi_{s}$, is sufficiently well obtained for most purposes ${ }^{1}$ by using the average value of the absolute temperature at which the superheating occurs and the specific heat $c_{p}$ as 0.48 . Thus,

$$
\phi_{s}=\int_{\theta_{0}}^{\theta} \frac{c_{p} d t}{\theta}=\frac{h}{\frac{\theta+\theta_{0}}{2}}=\frac{0.48\left(\theta-\theta_{0}\right)}{\frac{\theta+\theta_{0}}{2}}=0.96 \frac{\theta-\theta_{0}}{\theta+\theta_{0}} .
$$

The total entropy $\phi$ of a pound of superheated steam as measured from the assumed zero of entropy at $32^{\circ}$ Fahrenheit may then be written

$$
\phi=\phi_{s}+\phi_{v}+\phi_{s} . \quad \text {. . . . . . }
$$

59. Temperature-Entropy Chart for Superheated Steam. The $\theta-\phi$ chart for steam may now be extended into the superheated region to the right of the curve of constant steam weight. The increase of entropy due to superheating at constant pressure is found by equation (61), and lines of constant pressure $p_{1}, p_{2}$, and so on, are plotted

\footnotetext{
1 Stodola, in the 2d edition of "The Steam Turbine," uses 0.48. Cross interpolation in the table given on page 93 is usually unsatisfactory and not as accurate as a graphieal process. The tables of Marks and Davis give values of $v, \lambda$, and $\phi$ for intervals of $10^{\circ} \mathrm{F}$. for steam superheated at all the ordinary pressures of engineering.
} 
as in Figure 49. These lines, representing the increase in entropy at constant pressure, are essentially straight for small ranges of temperature. For large ranges and for high temperatures of superheat, they should be plotted, using values of the entropy as determined more exactly by use of the specitic heat values obtained by Knoblausch and Jakob.

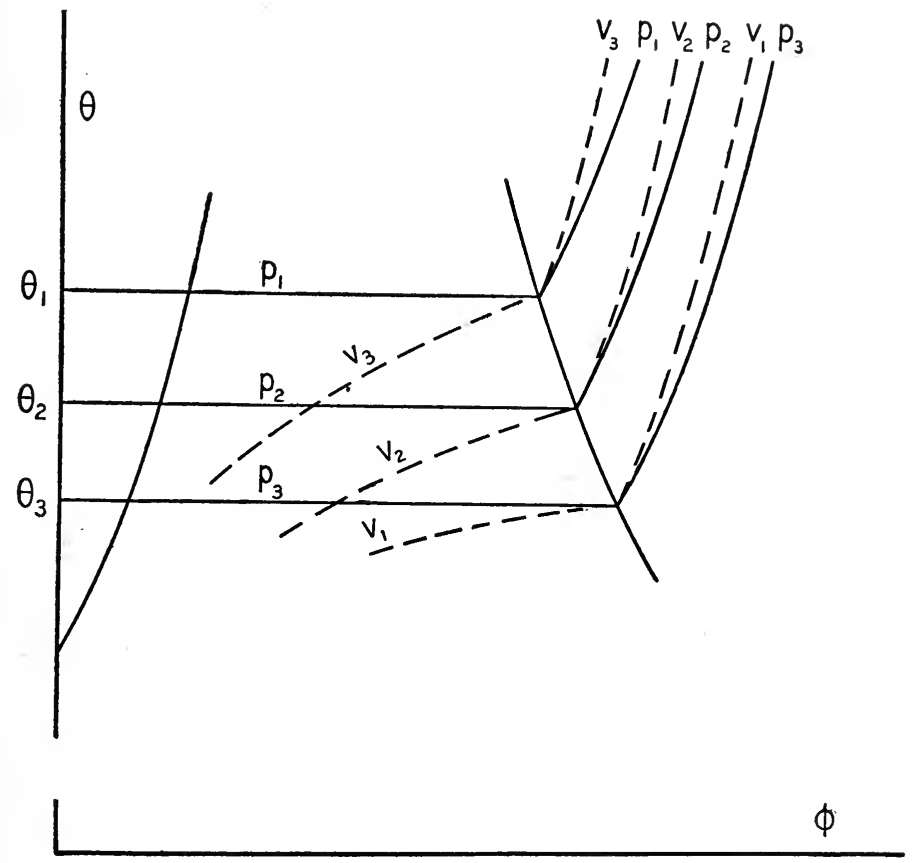

FIG. 49

Constant volume lines may also be extended into the superheated region by calculating for any assumed pressure and volume the corresponding temperature $\theta$ in accordance with either equation (57) or (58). The intersection of the line for constant pressure for the assumed value and the line of constant temperature for the calculated value $\theta$ gives a point on the desired constant volume line. The constant volume line might be drawn by a method similar to that employed in constructing the constant pressure line and using the increase in entropy for this condition calculated in a similar manner. were it not that the value of $c_{v}$ as 0.346 is not reliable. Constant pressure and constant volume lines are shown in Figure 49.

In a temperature-entropy chart constructed as above it is evident from Figure 49 that both the lines of constant pressure and the 
lines of constant volume are discontinuous at the curve of constant steam weight, since they are different functions of the temperature and the entropy in the wet steam and superheated steam regions. In the wet steam region the lines of constant pressure coincicle with the lines of constant temperature. The chart is therefore equally well a $\theta-\phi$ or a $p-\phi$ plot. In the superheated region it is merely a $\theta-\phi$ plot. Because of. the fact that steam is usually heated at constant pressure a $p$ - $\phi$ plot is sometimes convenient in this region. A chart constructed in this way is shown in Figure 42 on page 86.1 To indicate temperatures it is necessary to draw "quality" lines through those points representing steam of the same degree of superheat. Such lines would be everywhere equally distant from the curve of constant steam weight if the specific heat of superheated steam were constant. In the chart shown these lines are drawn for intervals of superheat equal to 20 degrees. The scale of temperatures given on the left-hand side obviously refers only to points within the wet steam region or on the dry steam line. The unequal scale of pressures on the right refers to any point of the plot. 'Such a plot is frequently spoken of as a temperature-entropy chart, but is one only for the region of wet steam and more rigorously should be called a pressure-entropy chart as stated above.

60. Intrinsic Energy of Superheated Steam. Let $p_{1}$ be the pressure upon a pound of superheated steam, $t_{1}$ (or $\theta_{1}$ absolute) be its temperature, and $v_{1}$ its specific volume as calculated by either equation (57) or (58). This superheated steam may be considered as derived at constant pressure $p_{0}$ (equal to $p_{1}$ ) from a pound of dry saturated steam at a saturation temperature $t_{0}$ (or $\theta_{0}$ ) corresponding to this pressure $p_{0}$. During the formation of this pound of dry steam a quantity of intrinsic energy has been added to it, which measured from water at $32^{\circ}$ Fahrenheit has been expressed in equation (50) as $\epsilon_{0}=\frac{1}{A}\left(q_{0}+\rho_{0}\right)$, where the subscript 0 indicates the value of any magnitude to which it is attached corresponding to the saturation temperature $t_{0}$. Since $\rho_{0}=r_{0}-A p_{0} u_{0}$ by equation (45) and since $u_{0}=s_{0}-\sigma$ by equation (44), it follows that the intrinsic energy of the pound of dry saturated steam may be written as

$$
\epsilon_{0}=\frac{1}{A}\left(q_{0}+r_{0}\right)-p_{1}\left(s_{0}-\sigma\right) . \quad . \quad . \quad .
$$

1 The entropy diagram constructed in connection with Problem 50 on page 120 is drawn both as a $p-\phi$ and as a $\theta-\phi$ plot to illustrate this difference. 
During superheating, at the pressure $p_{1}$, to the temperature $t_{1}$ the volume has increased to a value $v_{1}$, and there has been done an amount $p_{1}\left(v_{1}-s_{0}\right)$ of external work. Since the total heat added has been $h$, the increase $\epsilon_{1}-\epsilon_{0}$ in intrinsic energy due to superheating is

$$
\epsilon_{1}-\epsilon_{0}=\frac{1}{A} h-p_{1}\left(v_{1}-s_{0}\right) . \quad . \quad \text {. . . }
$$

The total intrinsic energy $\epsilon_{1}$ possessed by a pound of superheated steam in the condition indicated by the subscript 1 is then

$$
A \epsilon_{1}=q_{0}+r_{0}+h-A p\left(v_{1}-\sigma\right)=\lambda_{1}-A p\left(v_{1}-\sigma\right) .
$$

61. Isentropic Transformation. From the $\theta-\phi$ plot of Figure 50 it is evident that an isentropic or reversible adiabatic expansion of superheated steam may be considered in two parts; namely, an expansion entirely within the superheated region until the state $k$ represented by the intersection of the isentropic line $m n$ and the curve of constant steam weight, and a further expansion within the region of wet steam. During the first part of the expansion the quality, which may now be taken to mean degrees of superheat, that is $t-t_{o}$, decreases,. and during the second part of the expansion the quality as represented by $x$ decreases.

If the expansion is

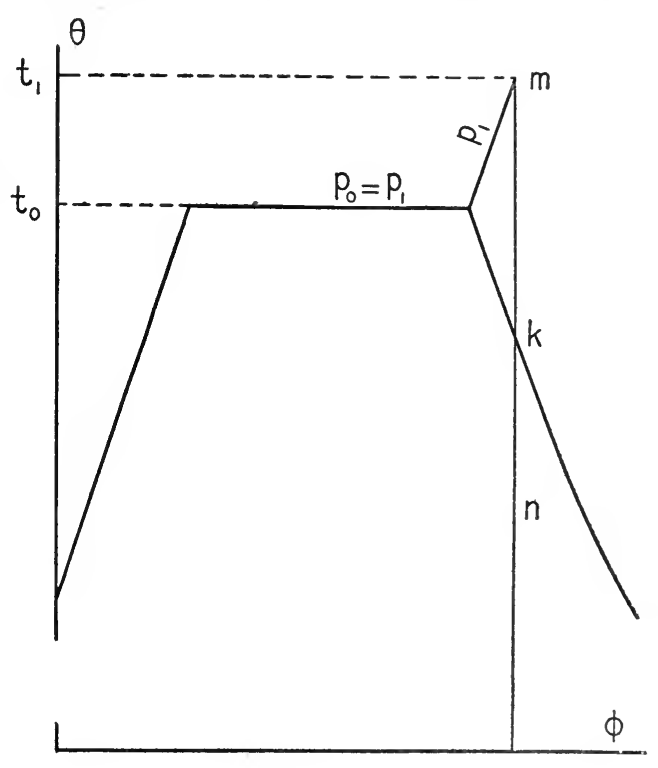

FIG. 50 wholly in the superheated region, the quality in the second state expressed in degrees superheat may most easily be found from a $\theta-\phi$ chart. It may, however, be found analytically by the method illustrated in Problem 38.

If the expansion, as is more usural, extends into the region of wet steam, the quality may be found for the second state by the following relation for constant entropy :

$$
\phi_{1}=\phi_{w}+\phi_{v}+\phi_{s}=\phi_{2}=\phi_{v_{2}}+x_{2} \phi_{v_{2}} . \quad . \quad \text {. }
$$


62. Superheating in Engineering Practice. The occasion for the use of superheated steam is evident in part from Problem 31 on page 87 . The steam supplied to the engine there described had a quality of over 99 per cent as it entered the engine. Due to the fact that heat was lost to the walls of the engine cylinder its quality lad dropped to 47 per cent at cut-off. During a portion of the expansion the transfer of heat to the cylinder walls continued, but during the latter part of the expansion some of this heat was returned to the mixture, reëvaporating some of the 53 per cent originally condensed during admission. During exhaust still further heat was abstracted from the cylinder walls to reëvaporate (at the condenser back pressure) the moisture left in the cylinder. As a result the temperature of the cylinder rises with the admission of steam and falls with its exhaust.

Consider for a moment that the entering steam is dry, if the quality at cut-off is $x_{1}$, then the intrinsic energy of the mixture has been reduced from $q_{1}+\rho_{1}$ to $q_{1}+x_{1} \rho_{1}$. The difference $\left(1-x_{1}\right) \rho_{1}$ then represents (neglecting all further changes due to heat transfers to the cylinder) a decrease in energy which should be available for the expansion. During the expansion still further heat energy is lost to the cylinder, and later returned at a lower availability. Of the heat lost to the walls during admission, part is returned at lower temperatures during expansion, and the rest in reëvaporation during exhaust. All of this energy of reëvaporation is of course rejected to the condenser.

It is evident, since a quantity of heat $q_{2}+r_{2}$, where the subscript 2 represents condenser conditions, is always to be rejected to the condenser, that as much as possible of the difference $q_{1}+r_{1}-q_{2}-r_{2}$ between the total heat supplied and rejected, must be converted into work. To accomplish this the mean temperature of the cylinder must be more nearly that of the entering steam. To prevent the loss at admission the steam may be supplied superheated by a number of degrees such that at cut-off the steam in the cylinder is dry. To prevent the loss during expansion the entering steam may be still further superheated so as to be dry at release. Then no heat will be abstracted from the cylinder walls to reëvaporate at the condenser back pressure. For any given engine it is of course a matter of experiment to determine how much superheat is required.

In general, it may be said, superheated steam reduces the amount of heat interchanged between the steam and the cylinder walls, and thereby reduces the weight of steam required per stroke of the 
engine for the same work output. Quoting from Ripper: ${ }^{i}$ "Superheating may be looked upon not as a means of obtaining a thermal efficiency with the engine in any way proportional to the temperature used in superheat, but as a device for realizing, or at least approaching, the full thermal efficiency of the saturated steam between the ranges of pressure used by the engine."

In the case of the steam turbine much of the advantage of dry steam is the consequent small friction in the turbine nozzle and passages. The presence of water materially increases this friction. Experiments by Stodola ${ }^{2}$ show that the friction increases as the density of the steam increases. Thus, for example, interpreting some experiments performed by Lewicki, Stodola says, the work of friction of a wheel in saturated steam with equal specific weight (i.e. density $\gamma$ ), equal size of wheel, and equal velocity is 1.3 times that in the air. The work of friction for a wheel in superheated steam at atmospheric pressure and $572^{\circ}$ Fahrenheit is the same as that for air.

\section{Résumé of Equations for Superheated Steam.}

Characteristic equations.

$$
\begin{aligned}
& p v=85.85 \theta-0.256 p \text {. . . . . . . . . . . . } \\
& p v=85.85 \theta-p(1+0.00000976 p)\left(\frac{150,300,000}{\theta^{3}}-0.0833\right) \text {. }
\end{aligned}
$$

Total heat contents.

$$
\lambda=q_{0}+r_{0}+h=q_{0}+r_{0}+c_{p}\left(\theta-\theta_{0}\right) \cdot \text {. } . . . \quad \cdot \quad \cdot
$$

Total intrinsic energy.

$\epsilon_{1}=\frac{1}{A} \lambda_{1}-p\left(v_{1}-\sigma\right) . \quad$. . . . . . . . . . . . .

Total entropy.

$$
\phi=\phi_{w}+\phi_{v}+\phi_{s}=\phi_{w}+\phi_{v}+\frac{c_{p}\left(\theta-\theta_{0}\right)}{\frac{\theta+\theta_{0}}{2}} \text {. . . . . }
$$

1 Ripper, "Steam Engine, Theory and Practice," 4th ed., p. 156 (Macmillan).

2 Lowenstein's translation, Stodola, "The Steam Turbine," 2d ed., pp. 135-140. 


\section{PROBLEMS AND SOLUTIONS: SUPERHEATED STEAM}

33. Specific volume. Find from equations (57) and (58) the volume occupied by two pounds of steam superheated $75^{\circ}$ Fahrenheit at a pressure of 135 pounds absolute.

Solution. From Table 3 , for $p=135$ we have $t_{0}=350^{\circ} \mathrm{F}$.

The absolute temperature of the superheated steam is $\theta=460+350+75=885$.

In eq. (58), substitution of this value of $\theta$ gives for the corrective term

$$
\left(\frac{150,300,000}{\theta^{3}}-0.0833\right)=0.134 \text {. }
$$

or

$$
\text { Hence } \begin{aligned}
v & =\frac{(85.85)(885)}{(135)(144)}-[1+0.00000976(135)(144)](0.134), \\
v & =3.91-0.16=3.75 \text { cubic feet. }
\end{aligned}
$$

Or the volume of two pounds of steam is 7.50 cubic feet.

By eq. (57), $v=\frac{(85.85)(885)}{(135)(144)}-0.256=3.91-0.26=3.65$ cubic feet.

The volume for two pounds is then 7.30 cubic feet.

The per cent of difference between the values calculated by equation (57) and (58)

is then

$$
\frac{3.75-3.65}{3.75} 100=2.7 \text { per cent. }
$$

34. Temperature of superheat. At 130.3 pounds gauge 3 pounds of steam occupy 10.5 culic feet. Find the degrees of superheat.

Solution. The volume per pound is 3.5 cubic feet. The pressure is 145 pounds absolute. The equation of Knoblauch, Linde, and Klebe (58) is capable of solution only by means of tedious approximations. The equation of 'Tumlirz (57) is here used.

$$
\theta=\frac{p v+0.256 p}{85.85}=\frac{(145)(144)(3.5+0.256)}{85.85}=913^{\circ} \text { Fahrenheit absolute. }
$$

From Table 3, the saturation temperature corresponding to a pressure of 145 pounds is $35.5 .8^{\circ} \mathrm{F}$. or $815^{\circ} \mathrm{F}$. absolute. The superheat is therefore $913-815=98^{\circ} \mathrm{F}$.

If greater accuracy is desired and justified by the precision with which $p$ and $v$ are known, this value of $\theta=913$ may be substituted in the corrective term of equation (58) and then that equation solved for $\theta$, considering the corrective term to be constant. Such a substitution gives $\theta=885$ and 70 degrees of superheat.

35. Specific heat of superheated steam. Find the heat required to superheat 2 pounds of steam 75 degrees at 135 pounds absolute.

Solution. For 135 pounds the saturation temperature is $350^{\circ}$ Fahrenheit.

From Table 4 , the specific heat is found to be approximately 0.558 , by cross interpolation between the four values.

\begin{tabular}{c|c|c} 
pounds & 113.8 & \multicolumn{1}{|l}{142.2} \\
\hline $392^{\circ} \mathrm{F}$. & 0.560 & 0.597 \\
\hline $482^{\circ} \mathrm{F}$. & 0.532 & 0.552
\end{tabular}


The heat required per pound is then $(0.558)(75)=41.8$ or 42 B.t.u. For two pounds it would require 84 B.t.u.

36. Total heat. Find the total heat contents of two pounds of steam superheated $75^{\circ}$ Fahrenheit at a pressure of 135 pounds absolute. How much heat is required to produce this steam from feed water at a temperature of $90^{\circ}$ Fahrenheit?

Solution. The total heat contents $\lambda_{1}$ of one pound of steam under the above conditions is $\lambda_{1}=q_{1}+r_{1}+h_{1}$.

From Table $3, q_{1}=321.5, r_{1}=869.8$, and $h_{1}$ from Problem 35 is 42 B.t. u. Hence $\lambda_{1}=1233.3$ B.t.u.

For feed water at $90^{\circ}$ Fahrenheit, $\lambda_{2}=q_{2}=58.1$ B.t.u.

Hence per pound $\lambda_{1}-\lambda_{2}=1175$ B.t.u. or 2350 B.t.u. for 2 pounds.

37. Entropy. Find the entropy for the superheated steam of Problem 36, and also the increase in entropy above that of the feed watel.

Solution. The entropy of superheat is

From Table 3,

$$
\phi_{s_{1}}=\frac{h}{\frac{\theta_{0}+\theta_{1}}{2}}=\frac{2(42)}{810+885}=0.0495 .
$$

Hence

For the feed water

Hence

$$
\begin{aligned}
\phi_{v_{1}} & =1.0741, \phi_{w_{1}}=0.5037 . \\
\phi_{1} & =1.6266 . \\
\phi_{2} & =0.1117 . \\
\phi_{1}-\phi_{2} & =1.5149 \text { or } 3.030 \text { for } 2 \text { pounds. }
\end{aligned}
$$

38. Quality. If the entropy of steam at 135 pounds absolute is 1.60, find its quality approximately.

Solution. Using the values of Problem 37, if $\phi_{1}=1.60$, then

$$
\phi_{s_{1}}=\phi_{1}-\phi_{v_{1}}-\phi_{v_{1}}=0.0251 \text {. }
$$

Assuming the specific heat as 0.48 , we have $h_{1}=0.48\left(\theta_{1}-\theta_{0}\right)$.

And

$$
\phi_{s_{1}}=\frac{2(0.48)\left(\theta_{1}-\theta_{0}\right)}{\theta_{0}+\theta_{1}}=0.0229 \text {. }
$$

Hence

$$
0.96 \theta_{1}-0.96 \theta_{0}=0.023 \theta_{0}+0.023 \theta_{1} \text {. }
$$

Substituting

$$
\theta_{0}=350+460=810 \text { as in Problem } 37,
$$

$$
\theta_{1}=\frac{0.983(810)}{0.937}=850, \text { corresponding to } 40^{\circ} \text { of superheat. }
$$

39. Quality after adiabatic expansion. If the steam of Problem 36 expauds adiabatically to 3 pounds back pressure (absolute), find the quality of the mixture.

Solution. From Problem 37,

$$
\phi_{1}=1.627 \text {. }
$$

From Table 3, for a pressure of 3 pounds, $\phi_{w_{2}}=0.201$, and

$$
\phi_{v_{2}}=1.684 \text {. }
$$

By equation 64,

$$
x_{2}=\frac{\phi_{1}-\phi_{w_{2}}}{\phi_{v_{2}}}=\frac{1.627-0.201}{1.684}=0.846 .
$$


40. Intrinsic energy. Find the intrinsic energy of the steam of Problem 36.

Solution. By equation 63 , the intrinsic energy $\epsilon_{1}$ is

$$
A \epsilon_{1}=q_{1}+r_{1}+h_{1}-A p_{1}\left(v_{1}-\sigma\right)=\lambda_{1}-A p_{1}\left(v_{1}-\sigma\right) .
$$

From Problem 33, the specific volume is 3.75 cubic feet, and from Problem 36 the total heat is $\lambda_{1}=1233$.

Therefore

or

$$
A \epsilon_{1}=1233-\frac{(135)(144)}{778}(3.75-\sigma)=1139 \text { B. t. u. per pound, }
$$

$$
\epsilon_{1}=(778)(1139)=885,000 \text { foot pounds per pound of steam. }
$$

For 2 pounds the energy is $1,770,000$ foot pounds.

41. Work of adiabatic expansion. Find the work done in the adiabatic expansion of Problem 39.

Solution. For state $1, \quad p_{1}=135, \quad t_{1}=425, \quad \epsilon_{1}=885,000 \mathrm{ft} . \mathrm{lb}$.

For state $2, \quad p_{2}=3, \quad x_{2}=0.846, \quad q_{2}=109.6, \quad \rho_{2}=946.4_{\text {s }}$ and hence $\quad \epsilon_{2}=\frac{1}{A}\left(q_{2}+x_{2} \rho_{2}\right)=(778)(911)=707,000 \mathrm{ft} . \mathrm{lb}$.

The work of the adiabatic expansion is

$$
w=\epsilon_{1}-\epsilon_{2}=885,000-707,000=178,000 \text { foot pounds per pound. }
$$

For 2 pounds of steam the work is 356,000 foot pounds.

42. Gain by superheating. The steam at cut-off in a certain engine cylinder has a quality of 80 per cent. It is found that super-

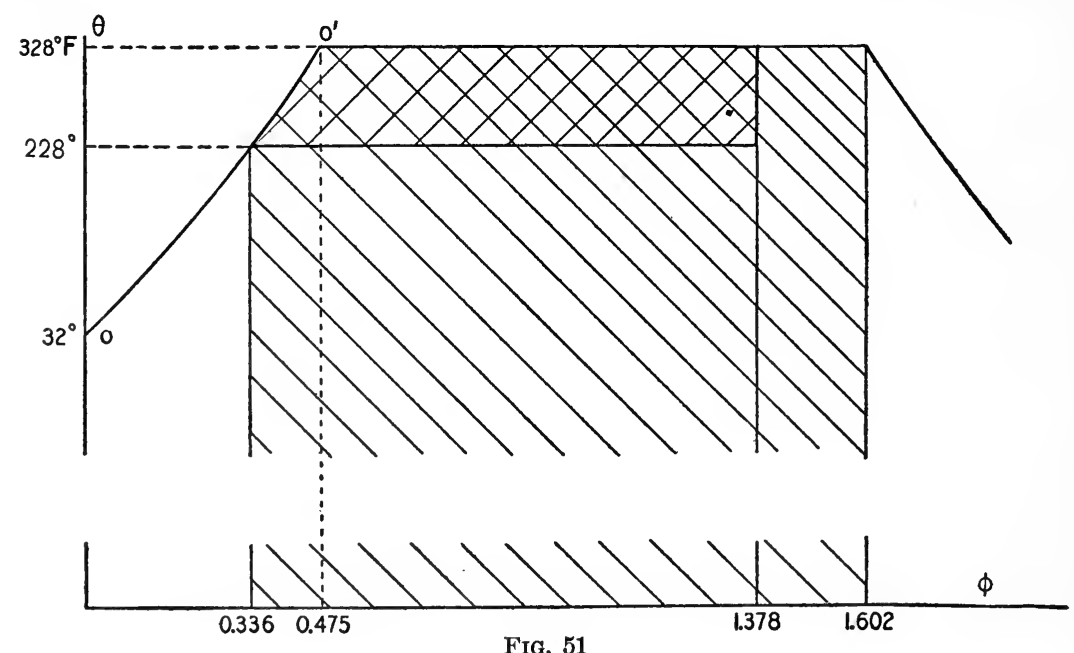

heating the steam 120 degrees makes the quality unity at cut-off. The entering steam is at a pressure of 100 pounds absolute. The 
back pressure is 20 pounds absolute. The specific heat may be taken at 0.52 . What is the gain in efficiency due to superheating? Plot the temperature entropy relations for both cases. Assume adiabatic expansion.

Solution. The $\theta-\phi$ plots for the condition of 80 per cent quality and 100 per cent quality are shown to scale in Figures 51 and 52 respectively. The useful work is repre-

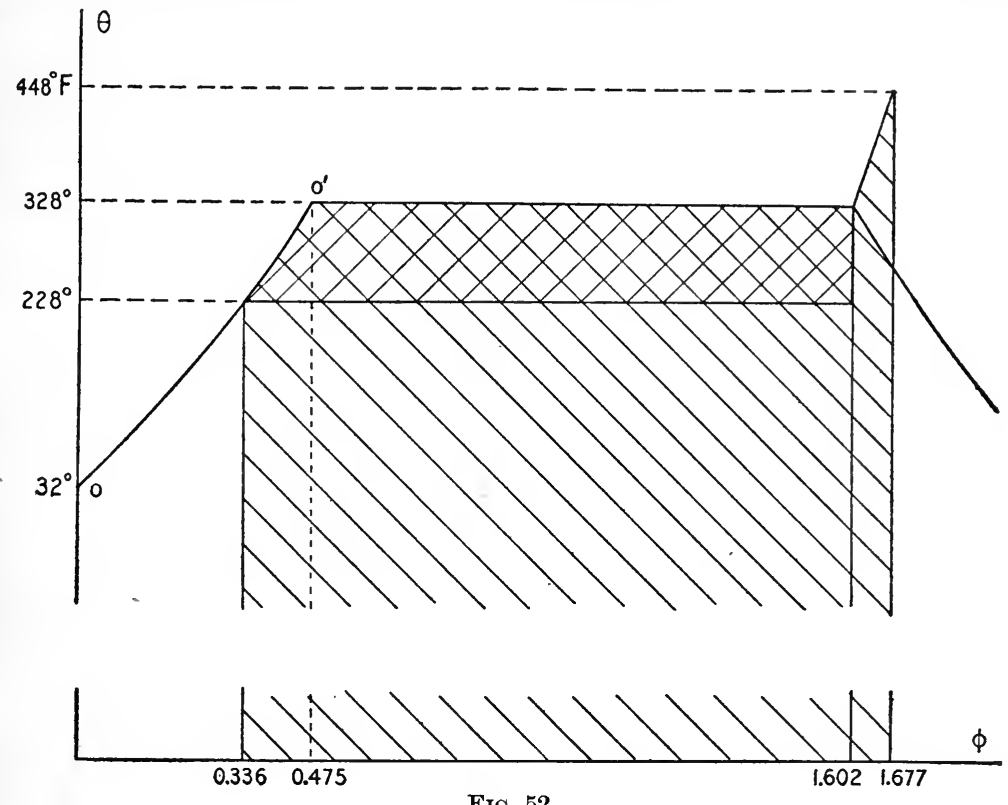

Fig. 52

sented in heat units by the doubly cross-hatched area in each case. The entire crosshatched area represents the heat supplied.

The useful work $\mathfrak{q}_{1}-\mathfrak{q}_{2}$ is found numerically by assuming the water line $o o^{\prime}$ a straight line and writing

$$
\mathfrak{q}_{1}-\mathfrak{q}_{2}=\left[\frac{1}{2}\left(\phi_{w_{1}}-\phi_{w_{2}}\right)+x_{1} \phi_{s_{1}}\right]\left(\theta_{1}-\theta_{2}\right) .
$$

The useful work for 80 per cent quality is then

$$
\mathfrak{q}_{1}-\mathfrak{q}_{2}=\left[\frac{1}{2}(0.4748-0.3362)+0.80(1.1273)\right](327.9-227.9)=97.1 \text { B.t.u. }
$$

Similarly, for 100 per cent quality,

$$
\mathfrak{q}_{1}{ }^{\prime}-\mathfrak{q}_{2}{ }^{\prime}=119.7 \text { B.t. u. }
$$

The input is $\mathfrak{q}_{1}=q_{1}+r_{1}-q_{2}=298.5+887.6-196.4=989.7$ B.t.u. for the first case. For the superheated steam $\mathfrak{q}_{1}{ }^{\prime}=q_{1}+r_{1}+h_{1}-q_{2}$ where $h_{1}$ is approximately (0.52) $(120)$ or 62.4 B.t.u. The input in the second case is therefore $1186+62=1052$ B.t.u.

The efficiency is then $\frac{97.1}{989.7}=9.81$ per cent for wet steam and $\frac{119.7}{1052}=9.60$ per cent for dry steam at cut-off.

For the conditions of the present problem the gain in efficiency due to superheating sufficiently to avoid cylinder condensation during admission is 1.54 per cent in 9.81 per cent or an increase of the efficiency of 15.7 per cent. 


\section{CHAPTER V}

\section{FLOW OF STEAM AND GASES}

64. Formula of de Saint-Venant. Consider a compressible body $B$ (Figure 53) acted upon by forces $F_{1}$ and $F_{1}^{\prime}$. Let $d z_{1}$ represent

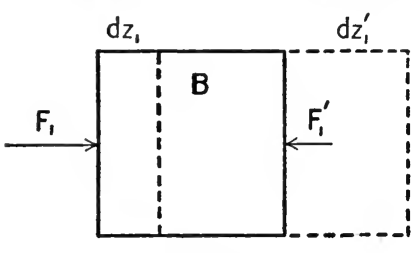

FIG. 53 the distance of motion of the point of application of $F_{1}$, and similarly $d z_{1}{ }^{\prime}$ represent the motion of the point of application of $F_{1}^{\prime}$. The motion $d z_{1}^{\prime}$ is of course negative if there is expansion as shown in the figure. Then the gain in energy of the entire mass $B$ is equal to the algebraic sum of the work of the acting forces, or $F_{1} d z_{1}-F_{1}^{\prime} d z_{1}^{\prime}$.

If we now consider two adjacent elements $B$ and $B^{\prime}$ of an aeriform body, as shown in Figure 54, it is evident that the force $F_{1}^{\prime}$, acting upon the element $B$, is equal and

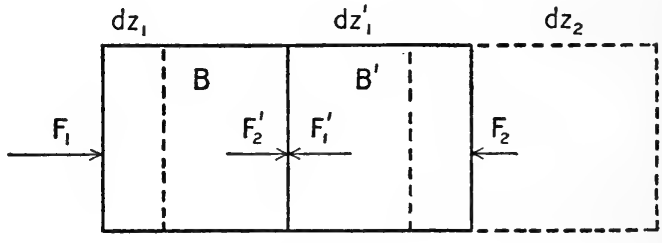

Fig. 54

opposite to the force $F_{2}{ }^{\prime}$ acting upon $B^{\prime}$. Hence, for this case, the total work done upon the entire body, $B$ plus $B^{\prime}$, is given by the expression

$$
d O=F_{1} d z_{1}-F_{2} d z_{2} . \quad \cdot \quad \cdot \quad \cdot \quad \cdot \quad \cdot \quad .
$$

This is the gain in energy of the entire mass, and will be represented in subsequent discussions by the symbol $d O$. The total change for an extended body will evidently be an expression of the same form as (i).

Consider a steady frictionless flow of steam in a non-conducting channel $C$, shown in Figure 55. The transformation of the steam will then be isentropic. Let the cross-section at $A$ be denoted by $a_{1}$ and at $B$ by $a_{2}$, the corresponding pressures on these areas by $p_{1}$ and $p_{2}$. Then, if $F_{1}$ and $F_{2}$ represent the forces acting upon $a_{1}$ and $a_{2}$ respectively,

$$
F_{1}=p_{1} a_{1} \text { and } F_{2}=p_{2} a_{2} . \quad \cdot \quad \cdot \quad \cdot \text {. }
$$


Since the flow is steady, there is the same amount of steam in pounds passing $A$ as is passing $B$ in the same time. If $M$ represents the weight of steam passing $A$ or $B$ in one second, and if the velocities at $A$ and $B$ are respectively $\mathfrak{v}_{1}$ and $\mathfrak{v}_{2}$ in feet per second, then this condition of steady flow may be expressed as

$$
\begin{aligned}
M v_{1} & =a_{1} \mathfrak{v}_{1} \\
\text { and } \quad M v_{2} & =a_{2} \mathfrak{v}_{2}, .
\end{aligned}
$$

where $v_{1}$ and $v_{2}$ are the specific volumes of the

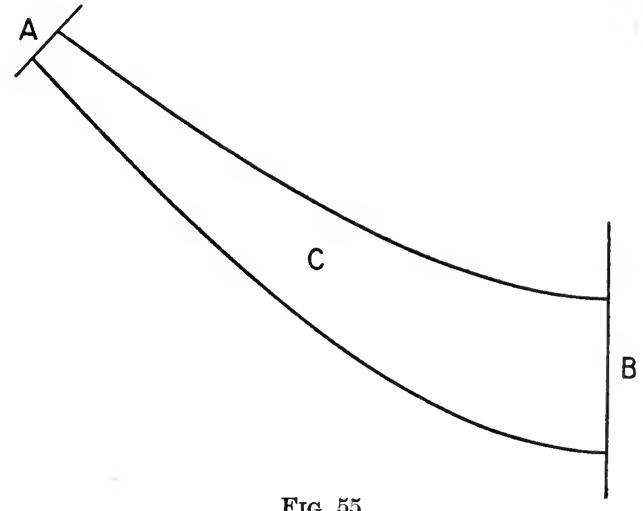

Frg. 55

steam in the states in which it passes $A$ and $B$ respectively. Equating $M$ from the two equations of (iii) gives

$$
M=\frac{a_{1} \mathfrak{b}_{1}}{v_{1}}=\frac{a_{2} \mathfrak{v}_{2}}{v_{2}}
$$

This equation is known as the "equation of continuity," and holds for any fluid in a condition of steady flow.

Substitution of this equation of continuity in the equation (i) $f_{(\circ}$ the increase of energy gives an equation expressing this increase $d O$ in terms of the pressure and specific volumes at $A$ and $B$. Thus, if we consider the flow for a small fraction of time $d \mathrm{t}$, then

$$
d z_{1}=\mathfrak{v}_{1} d \mathrm{t} \text { and } d z_{2}=\mathfrak{v}_{2} d \mathrm{t} . \text {. . . . }
$$

In the time $d \mathrm{t}$ the mass of steam passing $A$ or $B$ may be expressed as

$$
d m=M d \mathrm{t} \text {. }
$$

Hence, for this mass, $d m$, the equation of continuity (48) becomes

$$
d m=\frac{a_{1} \mathfrak{v}_{1} d \mathrm{t}}{v_{1}}=\frac{a_{2} \mathfrak{v}_{2} d \mathrm{t}}{v_{2}} .
$$

Substitution from equations (ii), (iv), (v), and (vi) in equation (i) gives

$$
d O=M\left(p_{1} v_{1}-p_{2} v_{2}\right) d \mathrm{t} .
$$

The total increase of energy expressed by equation (vii) is partly external kinetic energy of the entire mass of steam, $M d \mathrm{t}$, and partly 
intrinsic or molecular energy. Representing these components by $d K$ and $d E$ respectively, we have

$$
d O=d K+d E . \quad . \quad . \quad . \quad .
$$

The external kinetic energy expressed in terms of the gain in velocity of the entire mass of the steam is

$$
d K=\frac{M d \mathrm{t}}{2 g}\left(\mathfrak{v}_{2}^{2}-\mathfrak{v}_{1}^{2}\right) . \quad . \quad . \quad . \quad .
$$

The increase of intrinsic energy $d \boldsymbol{E}$ is equal to the external work done in compressing the steam. If there is expansion, as in the case considered, the increase in intrinsic energy is negative, and is numerically equal to the work done by the gas in expanding. Thus,

$$
\dot{d E}=-M d \mathrm{t} \int_{v_{1}}^{v_{2}} p d v . \quad . \quad . \quad . \quad . \quad .
$$

Substitution from equations (viii), (ix), and (x) in equation (vii) gives

$$
M d \mathrm{t}\left(p_{1} v_{1}-p_{2} v_{2}\right)=M d \mathrm{t} \frac{\mathfrak{v}_{2}^{2}-\mathfrak{v}_{1}^{2}}{2 g}-M d \mathrm{t} \int_{v_{1}}^{v_{2}} p d v . . \quad .
$$

Or dividing by $M d \mathrm{t}$ and transposing, we have

$$
\frac{\mathfrak{v}_{2}^{2}-\mathfrak{v}_{1}^{2}}{2 g}=p_{1} v_{1}-p_{2} v_{2}+\int_{v_{1}}^{v_{2}} p d v \ldots . .
$$

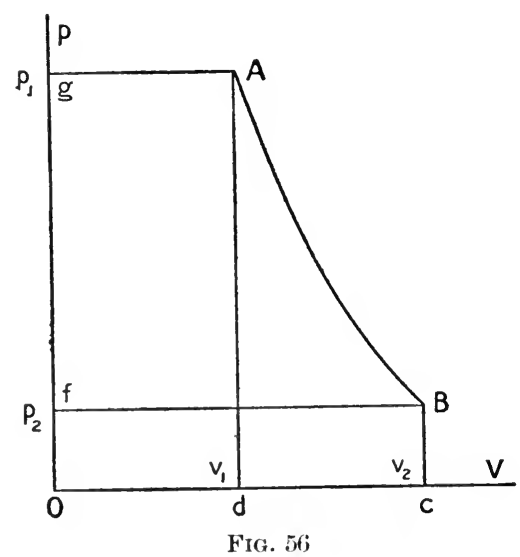
cific volumes at $A$ and $B$ if the frictionless adiabatic expansion may be taken as represented by an equation of the form

$$
p v^{n}=p_{1} v_{1}^{n}=p_{2} v_{2}{ }^{n} \cdot \text {. . . . . . }
$$


Then

Therefore

$$
\int_{p_{3}}^{p_{1}} v d p=\frac{n}{n-1}\left(p_{1} v_{1}-p_{2} v_{2}\right) . \quad \cdot \quad \cdot \quad \cdot \text { (xiv) }
$$

$$
\frac{\mathfrak{v}_{2}^{2}-\mathfrak{v}_{1}^{2}}{2 g}=\frac{n}{n-1}\left(p_{1} v_{1}-p_{2} v_{2}\right) . \quad . \quad . \quad . \quad .
$$

In this equation, which is due to de Saint-Venant, ${ }^{1} p$ and $v$ are specific pressures and specific volumes in pounds per square foot and cubic feet per pound, respectively. The kinetic energy term represents the increase in external kinetic energy per pound of steam. The exponent $n$ is given by Zeuner as $1.035+0.1 x$ for wet steam of quality $x$; that is, 1.135 for steam initially dry, and $n$ is 1.3 for superheated steam.

65. Alternative Expression for the Formula of de Saint-Venant. If in equation (viii) of the preceding section we substitute for $d K$ from equation (ix) of the same section, but for $d E$ write $\left(\epsilon_{2}-\epsilon_{1}\right) M d \mathrm{t}$, where $\epsilon_{1}$ and $\epsilon_{2}$ are the values of the intrinsic energy per pound of steam at $A$ and at $B$ respectively, we have

$$
M\left(p_{1} v_{1}-p_{2} v_{2}\right) d \mathrm{t}=M d \mathrm{t} \frac{\mathfrak{v}_{2}^{2}-\mathfrak{v}_{1}^{2}}{2 g}+\left(\epsilon_{2}-\epsilon_{1}\right) M d \mathrm{t},
$$

or dividing by Mit and transposing, we obtain for a pound of a mixture undergoing a frictionless adiabatic expansion the relation

$$
p_{1} v_{1}+\epsilon_{1}+\frac{\mathfrak{v}_{1}^{2}}{2 g}=p_{2} v_{2}+\epsilon_{2}+\frac{\mathfrak{v}_{2}^{2}}{2 g} .
$$

Equations (66) and (67) of Section 64 and equation (68) given above as well as the equation of continuity (65) hold equally well for all fluids. Thus if the fluid is a gas, the value of the exponent $n$ is of course $\kappa=\frac{c_{p}}{c_{v}}=1.4$ for diatomic mixtures,

$$
\frac{\mathfrak{v}_{2}^{2}-\mathfrak{v}_{1}^{2}}{2 g}=\frac{1.4}{0.4}\left(p_{1} v_{1}-p_{2} v_{2}\right) . \quad . \quad . \quad . \quad . \quad .
$$

If the fluid is incompressible as water, then $v_{1}=v_{2}$ and for an adiabatic change $\epsilon_{1}=\epsilon_{2}$,

whence

$$
\frac{\mathfrak{v}_{2}^{2}-\mathfrak{v}_{1}^{2}}{2 g}=\left(p_{1}-p_{2}\right) \sigma . . \quad . \quad . \quad . \quad . \quad . \quad .
$$

1 A more rigorous demonstration from which the above is derived is to be found in Lowenstein's translation of Stodola, "Steam Turbines," $2 d$ ed., pp. 4-8. 
For steam three cases may be distinguished; namely, (1) expansion through the channel from a condition of saturated steam of quality $x_{1}$ to a condition of quality $x_{2},(2)$ expansion from a superheated condition to a saturated condition of quality $x_{2}$, and (3) expansion entirely within the superheated region. For all these cases the expression for the gain in kinetic energy per pound of the steam mixture reduces to a simple form which will be derived in the following section.

66. Zeuner's Formula for Kinetic Energy. In equation (68) transposition gives

$$
\frac{\mathfrak{v}_{2}^{2}-\mathfrak{v}_{1}^{2}}{2 g}=\left(p_{1} v_{1}+\epsilon_{1}\right)-\left(p_{2} v_{2}+\epsilon_{2}\right) .
$$

Considering an expansion which takes place entirely within the region of wet steam, we have

$$
v_{1}=x_{1} u_{1}+\sigma, \text { and } v_{2}=x_{2} u_{2}+\sigma . \quad . \quad . \quad .
$$

Therefore, neglecting $\sigma$, which in general will make a difference of less than half of one per cent and substituting from equation (i) in equation (68), we may write

But

$$
\left(\epsilon_{1}+x_{1} p_{1} u_{1}\right)-\left(\epsilon_{2}+x_{2} p_{2} u_{2}\right)=\frac{\mathfrak{v}_{2}^{2}-\mathfrak{v}_{1}^{2}}{2 g} . . .
$$

$$
\epsilon_{1}=\frac{1}{A}\left(q_{1}+x_{1} \rho_{1}\right) \text { and } \epsilon_{2}=\frac{1}{A}\left(q_{2}+x_{2} \rho_{2}\right) . \quad \text {. . . . }
$$

Substituting from equation (iii) in equation (ii) and remembering that gives

$$
\lambda_{1}=q_{1}+x_{1} \rho_{1}+x_{1} A p_{1} u_{1} \text { and } \lambda_{2}=q_{2}+x_{2} \rho_{2}+x_{2} A p_{2} u_{2}, .
$$

$$
A \frac{\mathfrak{v}_{2}^{2}-\mathfrak{v}_{1}^{2}}{2 g}=\lambda_{1}-\lambda_{2} \cdot \quad \text {. . . . . . . }
$$

Similarly, consider the steam superheated in state 1 and wet in state 2. For superheated steam, equation (63) of Section 60 gives

$$
A \epsilon_{1}=q_{1}+r_{1}+c_{p}\left(\theta_{1}-\theta_{0}\right)-A p_{1}\left(v_{1}-\sigma\right) . . .
$$

Or neglecting $\sigma$ as before,

$$
\epsilon_{1}+p_{1} v_{1}=\frac{1}{A}\left[q_{1}+r_{1}+c_{p}\left(\theta_{1}-\theta_{0}\right)\right]=\frac{\lambda_{1}}{A} . \quad . \quad . \quad(
$$


Substituting for $\left(\epsilon_{1}+p_{1} v_{1}\right)$ from equation (vi) and for $\left(\epsilon_{2}+p_{2} v_{2}\right)$ from equations (iii) and (iv) in equation (ii) gives

$$
A \frac{\mathfrak{v}_{2}^{2}-\mathfrak{v}_{1}^{2}}{2 g}=\lambda_{1}-\lambda_{2}
$$

For the case of an expansion entirely within the superheated region similar reduction leads to the same equation as (71).

67. Kinetic Energy of an Irreversible Adiabatic Expansion. If, however, the decrease in total heat contents is not entirely converted into external energy, but is partly expended in external work of amount $w$, while some heat of amount $\mathfrak{q}$ is transferred from the substance to external bodies, we have to consider the general case of an expansion with heat changes and external work. Then equation (71) becomes

$$
\lambda_{1}-\lambda_{2}=A \frac{\mathfrak{v}_{2}^{2}-\mathfrak{v}_{1}^{2}}{2 g}+\mathfrak{q}+A w .
$$

If there is work done against friction, there must be added to the right-hand side of equation (71) a term $y$ representing the work per pound done against friction and expressed in heat units. If $\mathfrak{q}$ is zero, then the expansion is adiabatic; if $w$ is zero, then there is a free expansion; but if $y$ is not zero, then there is an adiabatic expansion similar to that described in the case of the "porous plug experiment" of Section 35, which is irreversible and during which there is an increase in entropy. This case of the irreversible expansion of steam in a non-conducting channel will be discussed in the latter part of the following section.

68. Loss of Kinetic Energy Due to Friction. If there is friction during the expansion from a pressure of $p_{1}$ to a pressure of $p_{2}$, the gain in kinetic energy will be less than it would have been for a similar frictionless expansion. Denote by $\mathfrak{b}_{2}{ }^{\prime}$ the final velocity for a How with friction for the same range of pressures as gives $\mathfrak{v}_{2}$ for a frictionless expansion. Then denoting by $l$ the loss of kinetic energy per pound of the mixture expressed in thermal units, we have

$$
l=A \frac{\mathfrak{v}_{2}^{2}-\mathfrak{v}_{1}^{2}}{2 g}-A \frac{\mathfrak{v}_{1}^{\prime 2}-\mathfrak{v}_{1}^{2}}{2 g}=A \frac{\mathfrak{v}_{2}^{2}-\mathfrak{v}_{2}{ }^{2}}{2 g} . . .
$$

Denoting by $\lambda_{2}{ }^{\prime}$ the total heat contents, after flow with friction, it is clear that

$$
l=\left(\lambda_{1}-\lambda_{2}\right)-\left(\lambda_{1}-\lambda_{2}{ }^{\prime}\right)=\lambda_{2}{ }^{\prime}-\lambda_{2}, \quad . \quad . \quad .
$$

where $\lambda_{1}$ and $\lambda_{2}$ have their former significance and refer to a frictionless flow. 
As the expansion takes place, a portion of the kinetic energy of the mixture is converted into heat by friction and immediately afterwards taken up by the mixture at a lower temperature and availability and again converted into kinetic energy. Since the heat equivalent of the friction work is thus continually being returned to the mixture, the actual loss in external kinetic energy due to the friction is less than the total work done against frictional forces. The heat corresponding to this friction work has been represented in the previous section by $y$.

This heat $y$ when returned to the steam or fluid in successive infinitesimal amounts $d y$ produces changes in the intrinsic energy of the mixture of amount to be represented by $d \epsilon$ and also produces an amount of external work of value $p d v$. That is,

But

$$
\begin{aligned}
& d y=A d \epsilon+A p d v \text {. . . . . . . . } \\
& A d(p v)=A p d v+A v d p \text {. . . . . . . } \\
& d y=A d \epsilon+A d(p v)-A v d p . \quad . \quad .
\end{aligned}
$$

In equation (iii) there may be substituted as in Section 66

Hence

$$
\begin{array}{r}
A d \epsilon+A d(p v)=A\left(\epsilon_{1}+p_{1} v_{1}\right)-A\left(\epsilon_{2}+p_{2} v_{2}\right)=d \lambda . . \\
d y=d \lambda-A v d p . \quad \text {. . . . . . . }
\end{array}
$$

By integration from state 1 to state 2

$$
y=\lambda_{2}-\lambda_{1}-A \int_{p_{1}}^{p_{2}} v d p, \quad . \quad . \quad . \quad .
$$

where equation (76) is a general relation for the total amount of friction work.

If now the expansion is frictionless and takes place from state 1 to state 2 , we have $y=0$ and

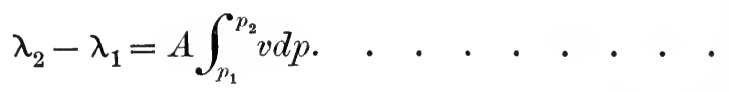

For the expansion with friction between the states 1 and $2^{\prime}$ at which the pressure is the same as for state 2 but at which the volume $v^{\prime}$ is different,

$$
y=\lambda_{2}^{\prime}-\lambda_{1}-A \int_{p_{1}}^{p_{2}^{\prime}} v^{\prime} d p . \quad . \quad . \quad .
$$

Subtracting equations (v) and (vi) gives

or,

$$
y=\lambda_{2}^{\prime}-\lambda_{2}-A\left(\int_{p_{1}}^{p_{2}^{\prime}} v^{\prime} d p-\int_{p_{1}}^{p_{2}} v d p\right)
$$

$$
y=\lambda_{2}{ }^{\prime}-\lambda_{2}-A \int_{p_{1}}^{p_{2}}\left(v^{\prime}-v\right) d p . \quad .
$$


In Figure 57 is shown the $p-v$ plot for these two expansions. From this it is evident that

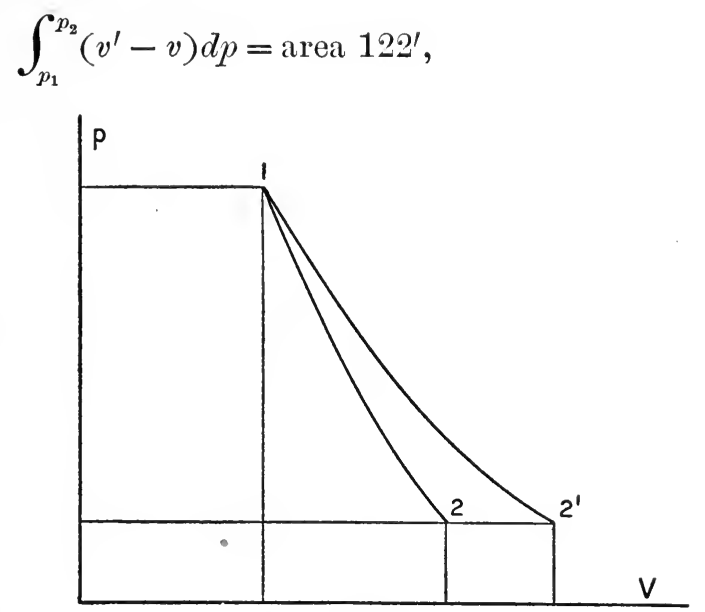

Fig. 57

or reversing the order of the integration and thus changing the algebraic sign,

$$
-\int_{p_{1}}^{p_{2}}\left(v_{1}^{\prime}-v\right) d p=+\int_{p_{2}}^{p_{1}}\left(v^{\prime}-v\right) d p=\text { area } 12^{\prime} 2 .
$$

Therefore

$$
y=\lambda_{2}{ }^{\prime}-\lambda_{1}+A \times \text { area } 12^{\prime} 2
$$

The total work done against friction is therefore larger than the loss of kinetic energy due to friction by the amount of work corresponding to the area on the $p$-v plot included by the line representing the actual expansion, the line representing an adiabatic expansion, and the line of constant final pressure.

The temperature entropy plot for these expansions is shown in Figure 58. For a frictionless adiabatic expansion the area $a b 12$ represents in heat units the increase in kinetic energy per pound. If all the kinetic energy of the expansion were converted into friction and this friction into heat, the line 13 representing the curve of constant total heat contents through 1 would be the path followed by the state point of the steam mixture. The area $23 \mathrm{fl}$ would then be equal to the area ab12. The path followed by the state point during the actual irreversible expansion through a channel like a turbine nozzle is some line between the constant heat line 13 and the reversible adiabatic 12 , such as $12^{\prime}$. For the path $12^{\prime}$ the 
friction work is $12^{\prime} e d$, of which $22^{\prime} e d$ represents the loss in kinetic energy $l$. The net increase of kinetic energy during an irreversible

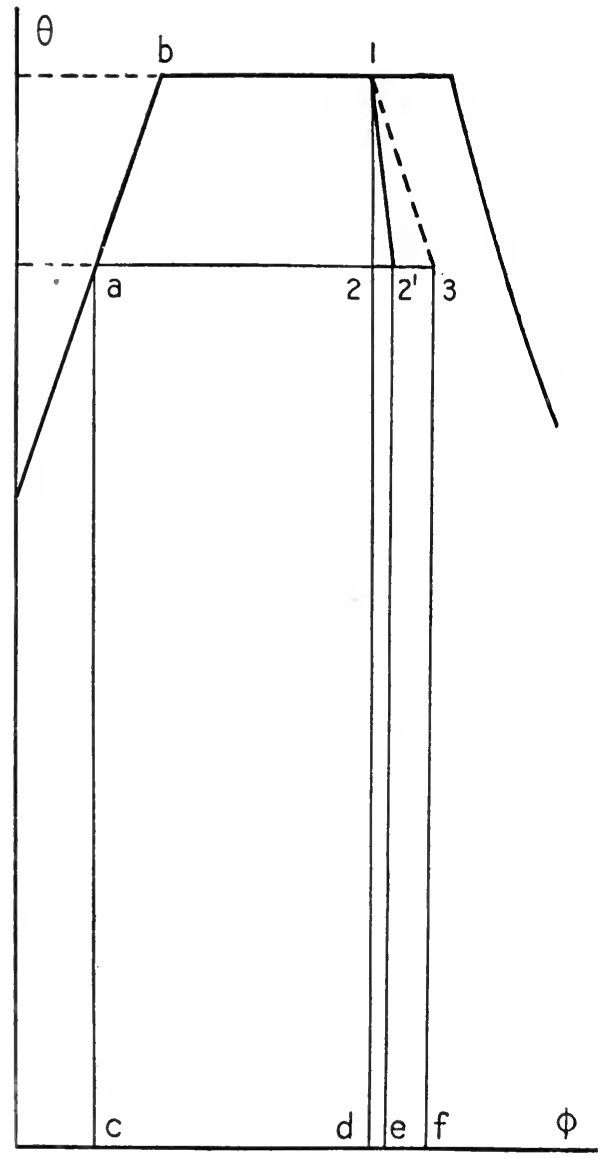

FIG. 58 adiabatic expansion is then expressed in heat units ky the area $12 a b$ diminished by the area $22^{\prime} e d$.

The loss $l$ is eomparatively small. For nozzles such as are used in turbines which are not more than 2 inches long $l$ is about 5 to 8 per cent of the energy which would be developed in a frictionless flow. For larger nozzles of length 4 to 6 inches and with diameters at the narrowest point of from one quarter to three eighths of an inch $l$ is from 10 to 15 per cent. ${ }^{1}$

69. Flow through an Orifice. If a fluid flows from a reservoir of such size that $\mathfrak{v}_{1}^{2}$ may be neglected in comparison with $\mathfrak{v}_{2}^{2}$ (as is the case in the flow of steam from a boiler into a pipe), then equations (i1) and (65) may be used to find the weight of fluid passing through the orifice if the second state be taken at the narrowest point or throat of the short tube or orifice. The reason for specifying that the second state shall be that at the throat is evident from the following experimental fact and also may be seen from the curves of Problem 5.3.

Experiments of Napier show that the weight of steam discharged through an orifice increases for a constant pressure $p_{1}$ with a reduction of the back pressure $p_{2}$ until $p_{2}$ is $0.58 p_{1}$, after which further reduction does not increase the weight discharged. In other words, 
the pressure in the orifice for steam is always such as to give a maximum value for the weight of steam discharged per second. For steam, therefore, the pressure at the orifice may be taken as $0.58 p_{1}$ provided that $p_{1}>1.73 p_{2}$.

Rankine therefore announced from his study of the experimental results obtained by Napier the following empirical formulas for calculating the flow of steam through an orifice. The symbols have the significance of the preceding discussion.

Where $p_{1} \geqq \frac{5}{3} p_{2}$,

$$
M=a \frac{p_{1}}{70}
$$

and

where $p_{1}<\frac{5}{3} p_{2}$,

$$
M=a \frac{p_{2}}{42} \sqrt{\frac{3\left(p_{1}-p_{2}\right)}{2 p_{2}}} . \quad \cdot \quad \cdot \quad \cdot
$$

It is to be noticed that these formulas of Napier and the assumption of the pressure in the throat as $0.58 p_{1}$ are only approximations. Since no flow is rigorously frictionless or adiabatic and the variations from these ideal conditions depend upon the orifice or tube employed when greater accuracy than about 2 per cent is required, the given nozzle should be calibrated by weighing the steam passing through it for various values of $p_{1}$ and $p_{2}$.

70. Fliegner's Formulas for the Flow of Air. For the flow of air through a rounded orifice, where the pressures are $p_{1}$ and $p_{2}$ on the two sides of the orifice and the area is $a$, the experiments of Fliegner show that the weight $M$ of air in pounds per second is given by the following formulas:

where $p_{1}>2 p_{2}$

where $p_{1}<2 p_{2}$

$$
M=0.530 a \frac{p_{1}}{\sqrt{\theta_{1}}} ; \quad \cdot \quad \cdot \quad \cdot \quad \cdot \quad \cdot
$$

$$
M=1.06 a \sqrt{\frac{p_{2}\left(p_{1}-p_{2}\right)}{\theta_{1}}}
$$

In these formulas $\theta_{1}$ is the absolute temperature Fahrenheit of the air in the reservoir. The pressure and the area are to be measured in any similar units, as either in pounds per square foot and in square feet, or the pressures in pounds per square inch and the area in square inches. 
71. Resumé of Equations for Flow of Fluids.

Equation of continuity.

$$
M=\frac{a \mathfrak{v}}{v}=\frac{a_{1} \mathfrak{v}_{1}}{v_{1}} \quad . \quad \cdot \quad \cdot \quad . \quad . \quad . \quad . \quad . \quad .
$$

Frictionless adiabatic flow of steam.

$$
A \frac{\mathfrak{v}_{2}^{2}-\mathfrak{v}_{1}^{2}}{2 g}=\lambda_{1}-\lambda_{2} . \quad \text {. . . . . . . }
$$

General equation.

$$
\lambda_{1}-\lambda_{2}=A \frac{\mathfrak{v}_{2}^{2}-\mathfrak{v}_{1}^{2}}{2 g}+\mathfrak{q}+y+A w . \quad . \quad . \quad .
$$

Friction work.

$y=l+A$ (area between isentropic and actual expansion paths

on a $p$-v plot). . . . . .

Loss of external K. E. due to friction.

$$
l=\lambda_{2}{ }^{\prime}-\lambda_{2} \cdot \quad \cdot \quad \cdot \quad \cdot \quad \cdot \quad \cdot \quad \cdot \quad . \quad . \quad . \quad .
$$

Napier's formulas for steam flow.

$$
\begin{aligned}
& M=a \frac{p_{1}}{70}, \text { where } p_{1} \geqq \frac{5}{3} p_{2} . \quad . \quad . \quad . \quad . \\
& M=a \frac{p_{2}}{42} \sqrt{\frac{3\left(p_{1}-p_{2}\right)}{2 p_{2}}}, \text { where } p_{1}<\frac{5}{3} p_{2^{\circ}}
\end{aligned}
$$

Fliegner's formula for air flow.

$$
\begin{aligned}
& M=0.530 a \frac{p_{1}}{\sqrt{\theta_{1}}, \text { where } p_{1}>2 p_{2} . \quad . \quad . \quad .} \\
& M=1.060 a \sqrt{\frac{p_{2}\left(p_{1}-p_{2}\right)}{\theta_{1}}}, \text { where } p_{1}<2 p_{2} .
\end{aligned}
$$

\section{PROBLENS AND SOLUTIONS}

43. Kinetic energy of steam. Given one pound of steam initially dry, issuing from a large reservoir under a pressure of 100 pounds absolute. Assume adiabatic expansion to a back pressure of 14.7 pounds absolute. Consider the velocity in the reservoir negligible. Find the final velocity $(a)$ using equation (67), (b) using equation (71); (c) find the error due to negleeting the term $\sigma\left(p_{1}-p_{2}\right)$. 
Solution. From Table 3, for 100 pounds, $q_{1}=298.5, r_{1}=887.6, \phi_{w_{1}}=0.4748$, $\phi_{v_{1}}=1.1273, s_{1}=4.432 ;$ for $p_{2}=14.7$ pounds, $q_{2}=180.3, r_{2}=969.7, \phi_{w_{2}}=0.3125$, $\phi_{v_{2}}=1.4441, s_{2}=26.78$.

Hence the quality after adiabatic expansion without friction is

$$
x_{2}=\frac{\phi_{w_{1}}+\phi_{v_{1}}-\phi_{w_{2}}}{\phi_{v_{2}}}=\frac{0.4748+1.1273-0.3125}{1.4441}=0.893 .
$$

(a) $v_{1}=s_{1}=4.432 ; v_{2}=x_{2} s_{2}$, approximately, or $(0.893)(26.78)=23.92$.

By equation (67),

$$
\frac{\mathfrak{v}_{2}^{2}}{2 g}=\frac{1.135}{0.135}[(100)(144)(4.432)-(14.7)(144)(23.92)]=111,100 \text { foot pounds. }
$$

Therefore

$$
\mathfrak{v}_{2}=2670 \text { feet per second. }
$$

$(b) \cdot \lambda_{1}=298.5+887.6=1186.1, \lambda_{2}=180.3+x_{2}(969.7)=1046.4$.

Hence, in equation (71), $J\left(\lambda_{1}-\lambda_{2}\right)=108,800$ foot pounds,

the term $\sigma\left(p_{1}-p_{2}\right)$ is of value $(0.016)(144)(100-14.7)=196$ foot pounds, and is negligible.

Therefore, neglecting this term, we have

or

$$
\frac{\mathfrak{b}_{2}{ }^{2}}{2 g}=108,800
$$

(c) The term neglected above evidently introduces an error of 0.2 per cent in the value of the kinetic energy, or 0.1 per cent in the value of the final velocity.

44. Kinetic energy of superheated steam. Given one pound of steam at 100 pounds absolute and $120^{\circ}$ Fahrenheit of superheat, find the final velocity acquired in expanding along a reversible adiabatic to a back pressure of 14.7 pounds absolute, $(a)$ by using equation (67), (b) by using equation (71), as in Problem 43.

Solution. From Table 3, the values of the constants for these pressures may be obtained, as given in the preceding problem. For $120^{\circ}$ of superheat $c_{p}$ may be taken as 0.53. Then

and

$$
\begin{gathered}
h_{1}=c_{p}\left(\theta_{1}-\theta_{0}\right)=0.53(120)=63.6 \text { B.t.u. }, \\
\phi_{s_{1}}=\frac{63.6}{\frac{1}{2}(788+908)}=0.0750 .
\end{gathered}
$$

The specific volume is found by equation (58) to be 5.27 cubic feet.

The quality after expansion is, then,

$$
x_{2}=\frac{\phi_{w_{1}}+\phi_{v_{1}}+\phi_{s_{1}}-\phi_{w_{2}}}{\phi_{v_{2}}}=\frac{0.4748+1.1273+0.0750-0.3125}{1.4441}=0.945 .
$$

(a) Since the expansion extends from the superheated into the saturated region, it is necessary to divide the expression of equation (67) for the increase of kinetic energy into two parts, one representing the increase while the steam is superheated and calculated by using $n=1.3$, and one representing the increase while the steam is saturated and calculated, as in Problem 43, by using $n=1.135$. This demands a knowledge of the pressure and volume corresponding to the intersection of the isentropic line 
with the curve of constant steam weight. This is most easily found from the $\theta-\phi$ chart of page 86 to be 40 pounds for the pressure and 10.5 cubic feet for the specific volume. It may be found approximately by using the values for the entropy at 30 and 50 pounds, and assuming that the rate of change of the entropy is proportional to the rate of change of the pressure. Such an interpolation gives the pressure as 39 pounds. The more accurate value of 40 pounds will be used in this solution. Substitution of these values in equation $(67)$ then gives

$$
\begin{aligned}
\frac{\mathfrak{\nu . 2}_{2}^{2}}{2 g}= & \frac{1.3}{0.3}[(100)(144)(5.27)-(40)(144)(10.5)] \\
& \quad+\frac{1.135}{0.135}[(40)(144)(10.5)-(14.7)(144)(25.3)] \\
= & 67,000+58,100=108,000 \text { foot pounds. }
\end{aligned}
$$

Hence $\mathfrak{v}_{2}=2830$ feet per second.

(b) Solution by equation (71) is similar to that of Problem 43 except that $\lambda_{1}$ is greater by the superheat $h_{1}$.

$$
\begin{aligned}
\lambda_{1} & =1186.1+63.6=1249.7, \quad \lambda_{2}=180.3+(0.945)(969.7)=1096.5 . \\
\frac{\mathfrak{v}_{2}^{2}}{2 g} & =J\left(\lambda_{1}-\lambda_{2}\right)=778(153.2)=119,200 \text { foot pounds. }
\end{aligned}
$$

Hence $\mathfrak{v}_{2}=2770$ feet per second.

Discussion. The deviation between solutions $(a)$ and $(b)$ is due to the inaccuracy

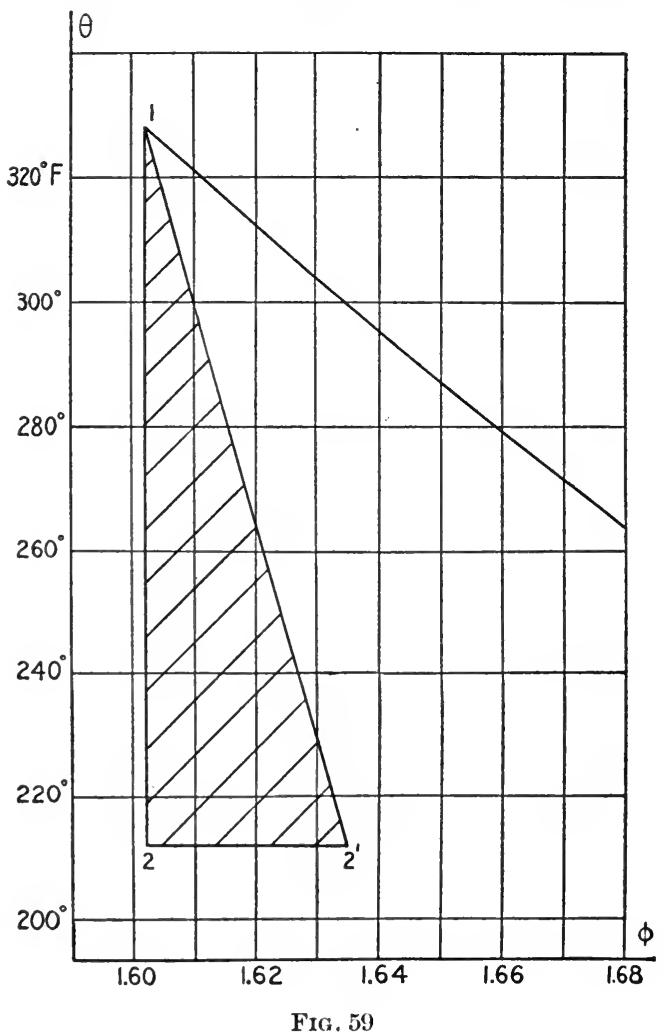
with which $n$ is accompanied. The method of $(b)$ is the more accurate and in general shorter of solution.

45. Loss due to friction. In Problem 43 the velocity for a frictionless adiabatic expansion has already been found. Taking the same values for the pressures but assuming a loss of 16 per cent of the energy available for increasing the kinetic energy, find the final velocity. Sketch the $\theta-\phi$ plot and find the total work done against friction. Assume the plot of the actual expansion line on the $\theta-\phi$ diagram to be a straight line.

Solution. The increase in kinetic energy of the steam for 
a reversible adiabatic has been determined as 108,800 foot pounds, and the final cuality as 0.893 . The loss, $l$, in kinetic energy is then $\frac{18}{100}(108,800)=17,400$ foot pounds $=22 \mathrm{~B}$.t.u. The absolute temperature corresponding to the back pressure is $212+460=672$ degrees. The inçrease in entropy due to the friction is then $\frac{22}{6}=$ 0.0328 and is represented in Figure 59 by the line $22^{\prime}$.

The area $12^{\prime 2}$ may now be found approximately by multiplying half this increase in entropy by the difference in temperature between points 1 and 2 ; that is,

area $12^{\prime} 2=\frac{1}{2}(0.0328)(328-212)=1.9$, or approximately 2 B. t. u.

The work done against friction, $y$, is by equation $(77)$ equal to the sum of this area and $l$. Hence $y=22+2=24$ B. t. u., or 18,700 foot pounds.

The final velocity is of course one half of 16 per cent smaller than that for a frictionless flow, since the energy available is 16 per cent smaller. Hence $\mathfrak{v}_{2}=24400$ feet per second.

46. Flow of air. Find the weight of air passing per second through a rounded orifice of area 0.1 square inch, if the pressures on the two sides of the orifice are 45 and 14.7 pounds absolute, $(a)$ by equation (67), $(b)$ by equation (80). The temperature of the air within the reservoir is $80^{\circ}$ Fahrenheit.

Solution. (a) The pressure $p_{t}$ in the throat, where the area is given above, is to be taken according to Fliegner's experiments as 0.577 of $p_{1}$, or for this case $p_{t}=(0.577)(45)$. The specific volume of the air in the reservoir is, by equation (13),

$$
v_{1}=\frac{80+460}{460} \frac{14.7}{45} 12.39=4.75 \text { cubic feet. }
$$

The specific volume after expansion is, by eq. (32),

$$
v_{2}=\left[\frac{45}{(0.577) 45}\right]^{\frac{1}{1.40}}(4.75)=7.02 \text { cubic feet. }
$$

Hence, by eq. (65),

$M=\frac{0.1}{(144)(7.02)} \sqrt{\frac{1.40}{0.40} 2 g[(45)(144)(4.75)-(0.577)(144)(7.02)]}=0.100$ pounds per second.

(b) By equation (80), since $\theta_{1}=80+460=540$,

$$
M=(0.530)(0.1) \frac{45}{\sqrt{540}}=0.102 \text { pound per second. }
$$

47. Napier's formula. Given steam of 95 per cent quality expanding from 120 pounds absolute to atmospheric pressure of 14.7 pounds through an orifice of 0.1 square inch area. Assume the pressure in the throat to be $0.58 p_{1}$ or 70 pounds absolute. Find the weight of steam per second, (a) by equation (71), (b) by equation (78).

Solution. (a) From Table 3, for 120 pounds, $q_{1}=312.3, r_{1}=876.9, \phi_{w_{1}}=0.4922$, $\phi_{v_{1}}=1.0951$. For $p_{t}=70$ pounds, $q_{t}=272.9, r_{t}=906.6, \phi_{v_{t}}=0.4418, \phi_{v_{t}}=1.1892$, $s_{t}=6.199$. 
Hence And

$$
x_{t}=\frac{0.4922+(0.95)(1.0951)-0.4418}{1.1892}=0.917, \quad v_{t}=(0.917)(6.199)=5.69 .
$$

$\lambda_{1}-\lambda_{t}=312.3+(0.95)(876.9)-272.9-(0.917)(906.6)=41.1$.

Then

$$
M=\frac{a_{t} \mathrm{\Downarrow}_{t}}{v_{t}}=\frac{0.1 \sqrt{2 g 778(41.1)}}{(144)(5.69)}=0.175 \text { pound per second. }
$$

(b) $\quad M=\frac{(0.1)(120)}{70}=0.172$ pound per second.

48. Napier's formula. Compare the weight of steam of quality 95 per cent passing through an orifice 0.1 square inch in area from an initial pressure of 20 pounds absolute to atmospheric pressure of 14.7 pounds, as calculated (a) by equation (71), (b) by equation (79).

Solution. (a) From Table 3, for $p_{1}=20$ pounds, $q_{1}=196.4, r_{1}=959.4, \phi_{w_{1}}=0.3362$, $\phi_{v_{1}}=1.3957$. For $p_{2}=14.7$ pounds, $q_{2}=180.3, r_{2}=969.7, \phi_{w_{2}}=0.3125, \phi_{v_{2}}=1.4441$, $s_{2}=26.78$.

Hence

$$
\begin{aligned}
x_{2} & =\frac{0.3362+(0.95)(1.3957)-0.3125}{1.4441}=0.9345 \text { or } 0.934 . \\
v_{2} & =(0.934)(26.78)=25.0 . \\
\lambda_{1}-\lambda_{2} & =196.4+(0.95)(959.4)-180.3-(0.934)(969.7)=21.9 \text { B.t.u. }
\end{aligned}
$$

Therefore

$$
M=\frac{0.1 \sqrt{2 g 778(21.9)}}{(144)(25.0)}=0.0291 \text { pound per second. }
$$

(b) $\quad M=\frac{0.1(14.7)}{42} \sqrt{\frac{3(20-14.7)}{2(14.7)}}=0.0257$ pound per second.

49. Injector (non-lifting). An injector, as the name implies, is a device for injecting water into a boiler against the boiler steam pressure. This is accomplished by a mechanism the principles of which are evident from Figure 60. To start the injector, steam is

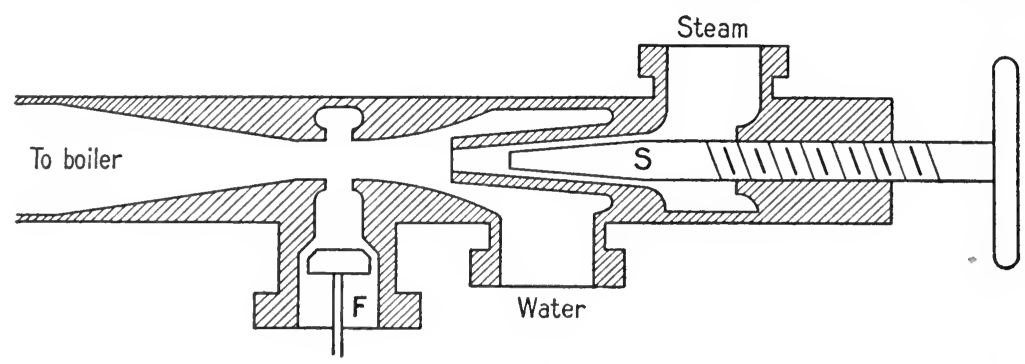

FIG. 60

allowed to flow from the steam delivery pipe through the injector and out of the overflow $F$, thus blowing out any condensed water 
and insuring practically dry steam. The steam valve $S$ is then turned off and the water valve turned on. When the water appears at the overflow the steam valve and the valve in the delivery tube to the boiler are both turned on. The entering steam is condensed by contact with the colder feed water and converts some of its heat energy into mechanical kinetic energy, forcing feed water and the condensed steam into the boiler. The partial vacuum formed by the steam and water rushing by the overflow valve draws it shut.

Calculate the number of pounds of water forced into a boiler against a pressure of 120 pounds absolute by one pound of steam from the same boiler. Neglect the velocity of the entering water. Assume the steam 98 per cent dry, the temperature of the water supplied to the injector as $70^{\circ}$ Fahrenheit, and the temperature of the water leaving the injector as $170^{\circ}$ Fahrenheit. Assume that the kinetic energy of the entire mass of water leaving the injector is one per cent of the total heat contents of one pound of steam.

Solution. Let there be $m$ pounds of feed water entering the injector for every pound of steam. The total energy of the entering water plus that of the entering steam must equal the total energy of the two combined as they leave the injector. That is,

$m \lambda_{\text {water }}+\mathrm{K} . \mathrm{E}_{\text {water }}+\lambda_{\text {steam }}+\mathrm{K} . \mathrm{E}_{\text {steam }}=(1+m) \lambda_{\text {(water+steam) }}+\mathrm{K} . \mathrm{E}_{\text {(water+steam) }}$, where

$\lambda_{\text {water }}=39$ B.t. u. ; K. E. water is negligible ; $\lambda_{\text {steam }}=q+x r=1172$ B.t.u. per pound.

K. E.(water+steam) $=12$ B. t. u. ; and $\lambda_{\text {(water+steam) }}=138$ B. t. u. per pound.

Hence $39 m+1172=(1+m) 138+12$ or $m=10$ pounds approximately.

For practical purpose it is sufficient to neglect all kinetic energy terms and write

$$
\begin{aligned}
m=\frac{(x r)_{s}+q_{s}-q_{w+s}}{q_{w+s}-q_{w}} & =\frac{1172-138}{138-39} \\
& =10 \text { pounds approximately. }
\end{aligned}
$$

50. Peabody throttling calorimeter. The Peabody throttling calorimeter employs the phenomena of an irreversible adiabatic expansion in the determination of steam quality. It consists of a reservoir $A$ shown in Figure 61 to which the steam of which the quality is desired is admitted by a small pipe $b$. A pressure gauge at $f$ and a thermometer at $e$ record the temperature and pressure of the steam after it has expanded to fill the reservoir. The valve $d$ admits of the free passage of steam from the calorimeter. The entire

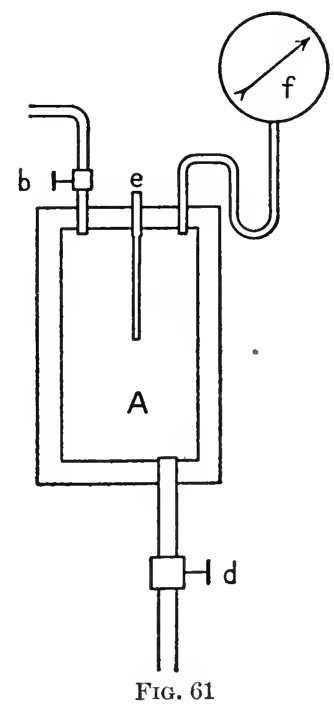


reservoir is surrounded by a heat insulator. For a test, the valve $b$ which connects the reservoir with the main steam pipe, in which it is desired to know the quality, is opened slightly, and thus admits steam throttled down to a pressure of 5 to 15 pounds above that of the atmosphere to which $d$ is connected.

If the boiler pressure is $p_{1}=80.3$ pounds gauge, the pressure $p_{2}$ within the calorimeter is 5.3 pounds gauge, the atmospheric pressure is 14.7 pounds, and the temperature within the calorimeter is $578^{\circ}$ Fahrenheit, find the quality of the steam. Sketch the temperature entropy diagram for this process.

Solution. There is assumed to be an irreversible adiabatic expansion, in which as fast as the heat of the entering steam is converted into kinetic energy of motion, this energy is transformed into work of friction and eddy currents, and is returned at a slightly lower temperature to the steam. The change thus takes place at constant heat contents Hence $\lambda_{1}=\lambda_{2}$. If the steam is nearly saturated, the constant heat line will take it into the superheated region for lower temperatures and pressures. Hence we have

$$
\lambda_{1}=q_{1}+x_{1} r_{1}=\lambda_{2}=q_{2}+r_{2}+c_{p}\left(t-t_{0}\right),
$$

where $t_{0}$ is the saturation temperature corresponding to the pressure $p_{2}$, and $t$ is the temperature as recorded by the thermometer in the calorimeter.

Hence, using 0.48 for $c_{p}$ and substituting the proper values for $q$ and $r$ from Table 3 , we have

$$
x_{1}=\frac{q_{2}+r_{2}+c_{p}\left(t-t_{0}\right)-q_{1}}{r_{1}}=\frac{196.4+959.4+0.48(278-228)-294.6}{890.5}=0.994 .
$$

The temperature-entropy plot is shown in Figure 62, where are plotted to scale the corresponding values of the entropy as found from Table 3 for the constant steam weight line, and by calculation for the curve of constant heat contents equal to $\lambda_{1}$.
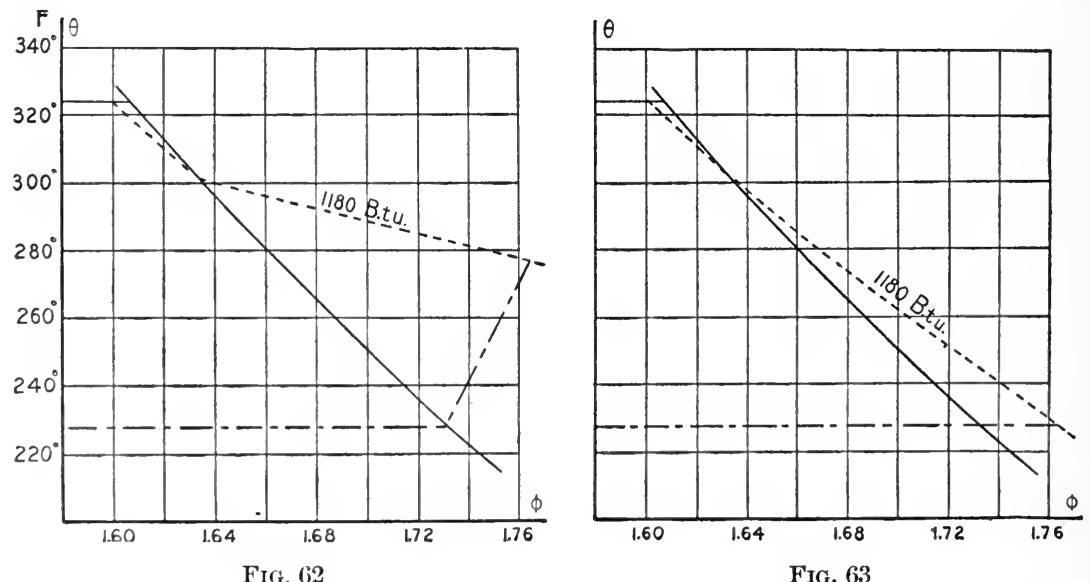

FIG. 63

To illustrate more fully the difference in appearance between the $\theta-\phi$ and the $p$ - $\phi$ diagrams discussed on page 96 , there is shown in Figure 63 to the same scale as used above the $p$ - $\phi$ plot for this process. 
51. Limits of throttling calorimeter. If the valve $d$ of the calorimeter described in Problem 50 is connected not to the atmosphere but to the exhaust pipe of the engine so that the back pressure is 3 pounds absolute, find the largest amount of priming (lowest value of $x$ ) that can be measured with the calorimeter for a boiler pressure of 85 pounds absolute. Neglect probable and observational errors in the thermometer.

Solution. At the back pressure of 3 pounds the steam must in the limiting case be just at the point of being superheated. Hence putting $c_{p}\left(t-t_{x}\right)$ equal to zero in the equation for $x_{1}$ derived in Problem 50 and substituting for $q$ and $r$ from Table 3, we have

$$
x_{1}=\frac{109.6+1012.2-286.5}{896.6}=0.9315 .
$$

For this pressure the calorimeter can then be used to determine the steam quality down to 93.15 per cent or 6.85 per cent of priming. The range of the apparatus is evidently extended by connecting the outlet to the condenser and thus using a lower back pressure.

52. Throttle control. A small non-condensing engine uses steam at 50 pounds absolute, receiving it through a throttle valve from a header containing dry steam at 200 pounds absolute. ${ }^{1}$ The atmospheric pressure is $\mathbf{1 4 . 7}$ pounds. Find the loss in availability of the steam in B.t.u. per pound due to throttling.

Solution. During throttling heat is converted into kinetic energy and then by friction into heat as in the calorimeter of Problem 50. The $\theta-\phi$ diagram for throttling is similar to that of Problem 50.

If a quantity of heat $d \mathfrak{Q}$ is received from a source at a temperature of $\theta$, and if the lowest available temperature is $\theta_{0}$, the Carnot efficiency between these two temperatures represents the fraction of $d \mathfrak{Q}$ which is available for useful work. This available portion of $d \mathfrak{D}$, the heat received from the hot source, has been termed its "motivity" by Lord Kelvin. ${ }^{2}$ Now the heat possessed by a mass of steam has been received at a series of temperatures ranging in the boiler from that of the feed water as it enters to its final temperature. At each of these temperatures the motivity or availability is given by an expression of the form $\frac{\theta-\theta_{0}}{\theta} d \mathfrak{O}$. The availability of a given mass in any state, as 1 , is then the sum of all the motivities due to the addition of the several infinitesimal amounts of heat $d \mathfrak{Q}$ at these various temperatures. That is, the motivity in state 1 is

$$
\int_{0} \frac{\theta-\theta_{0}}{\theta} d \mathfrak{Q}=\mathfrak{\Omega}_{1}-\theta_{0} \phi_{1}
$$

Similarly, the motivity in any other state, as 2 , is of the form $\mathfrak{Q}_{2}-\theta_{0} \phi_{2}$. The difference in avalability of the substance at these two states is then

$$
\mathfrak{Q}_{1}-\mathfrak{Q}_{2}+\theta_{0}\left(\phi_{2}-\phi_{1}\right),
$$

1 The conditions here chosen are not such as would ordinarily enter into good engineering practice. They are selected so that $\phi_{2}-\phi_{1}$ is large as compared to the precision with which $\phi_{1}$ or $\phi_{2}$ is known.

2 See Preston, “ Heat,” pp. 629, 630. 
an expression which for the condition of constant heat contents required by this particular problem reduces to

$$
\theta_{0}\left(\phi_{2}-\phi_{1}\right) \text {. }
$$

The lowest temperature for this problem is that at which the steam is rejected, or $212^{\circ}$ Fahrenheit. The absolute temperature $\theta_{0}$ corresponding to this is 672 . The entropy $\phi_{1}$ is found directly from Table 3 to be 1.5459.

In order to find the entropy in state 2 it is necessary to make use of the condition that the heat contents remain constant and solve for the temperature as follows :

Therefore

$$
\begin{aligned}
& \lambda_{1}=q_{1}+r_{1}=354.3+843.5=1197.8 \\
& \lambda_{2}=q_{2}+r_{2}+c_{p}\left(t_{2}-t_{0}\right)=250.4+922.8+c_{p}\left(t_{2}-281\right)=1173.2+h .
\end{aligned}
$$

$$
h=24.6 ; t_{2}-t_{0}=51.2 . \quad \phi_{8}=\frac{24.6}{460+\frac{1}{2}(281+332)}=0.0324 .
$$

The entropy in the saturated condition is found from the tables, hence the total entropy $\phi_{2}$ is $1.6581+0.0324=1.6905$. The loss in availability per pound is then

$$
672(1.6905-1.5459)=97.2 \text { B.t. u. }
$$

53. Nozzle area. Plot to a scale of pressures for abscissas and ordinates of B.t.u. the energy $\lambda_{1}-\lambda$ available for increasing the kinetic energy of a mixture of steam expanding adiabatically without friction from 150 pounds absolute and $75^{\circ}$ Fahrenheit of superheat to a back pressure of 6 pounds absolute. Plot also the velocity during this expansion. Plot the area in square inches of a nozzle to discharge one pound per second.

Solution. From Table 3 , for 150 pounds, $q_{1}=330.0, r_{1}=863.0$. For 75 degrees of superheat, use $c_{p}=0.57$. Hence $h_{1}=43$. Therefore $\lambda_{1}=1236$. $\phi_{w_{1}}=0.5142$, $\phi_{v_{1}}=1.0551$, and $\phi_{s_{1}}=0.0503$. Hence $\phi_{1}=1.6196$ or 1.62 approximately.

The values of the heat contents for other pressures may be found either by calculation or they may be read directly from the pressure-entropy chart of page 86 . In this solution they are read directly from the chart, ${ }^{1}$ and also the values of the specific volumes corresponding. The velocity is then found by equation (71) and the requisite area by equation (65), which may be written

$$
\text { area in square inches }=\frac{(144)(\text { specific volume })}{\text { velocity }} \text {. }
$$

The desired values are plotted in Figure 64 .

54. Turbine nozzle. A 300 horse-power De Laval turbine uses 18 pounds of steam per horse-power hour. There are twelve nozzles supplying steam to the turbine wheel. The steam is at 150 pounds absolute and 75 degrees of superheat, and it expands to a back pressure of 2 pounds absolute. Find the diameters of the nozzle at the throat and at the exit end necessary to deliver the requisite amount

1 For the purposes of this calculation the diagram proposed by Professor Mollier and named after him is most convenient. In it ordinates are $\lambda$ 's and abscissas are $\phi$ 's. Lines of constant pressure and of quality are plotted. Such a diagram is contained in the Tables of Marks and Davis. 
of steam : (a) for frictionless adiabatic expansion, (b) for 15 per cent energy loss by friction. For the throat pressure use $p_{t}=0.58 p_{1}$.

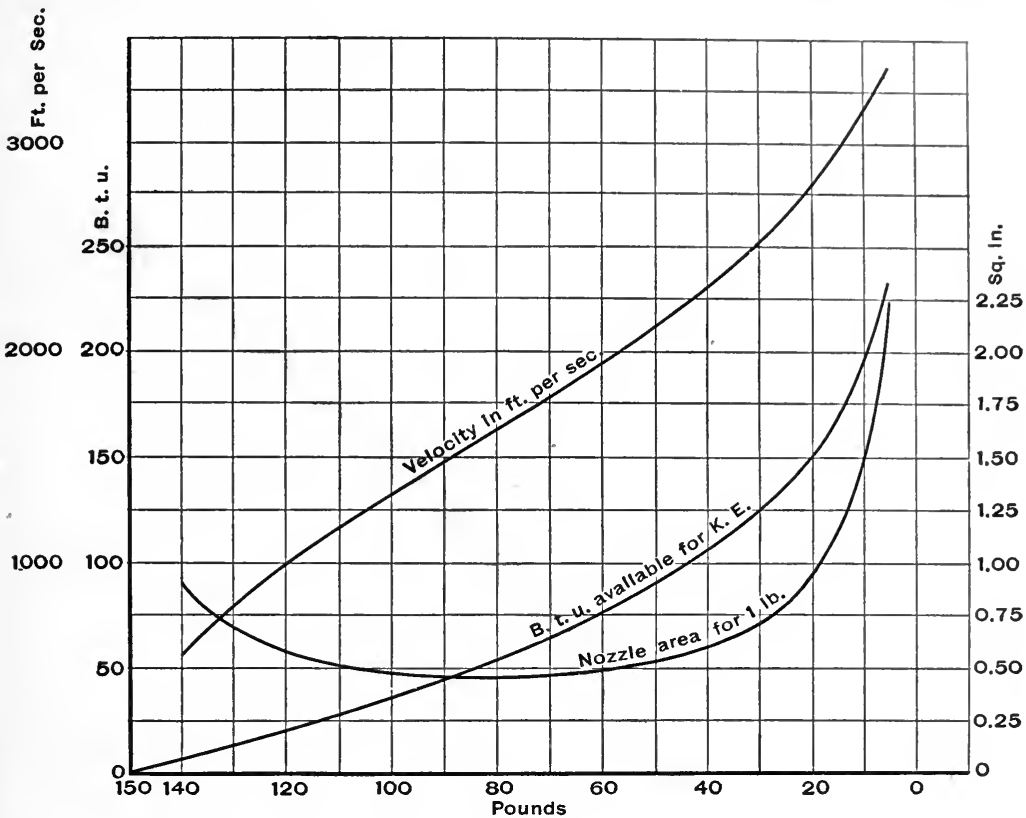

Fig. 64

Solution. (a) Each nozzle must deliver $\frac{(300)(18)}{(3600)(12)}$, or 0.125 pound per second.

The quality in the throat for adiabatic expansion is found as follows. The entropy is 1.6196 in the initial condition as found in Problem 53. For the throat pressure $\left(\phi_{w}\right)_{t}=0.4617$ and $\left(\phi_{v}\right)_{t}=1.1516$. Hence $\left(\phi_{s}\right)_{t}$ must equal 0.0063 . Since the saturation temperature is $318^{\circ}$ Fahrenheit, for the throat pressure of 87 pounds, the quality is 10 degrees of superheat (using $c_{p}=0.48$ ).

By equation (57) the specific volume is

$$
v_{t}=\frac{(85.85)(788)}{(87)(144)}-0.256=5.13 .
$$

The total heat content at the throat is $(\lambda)_{t}=288.2+895.3+4.8=1188.3$.

The total heat contents in the initial condition were found in Problem 53 to be $\lambda_{1}=1236$.

The velocity in the throat is then

$$
\mathfrak{v}_{2}=\sqrt{2 g 778(1236-1188.3)}=1545 \text { feet per second. }
$$

The requisite area in the throat must then be

$$
\frac{(144)(5.13)}{1545}=0.479 \text { square inch per pound of steam. }
$$

But for the turbine nozzle of the problem only 0.125 pounds per second are to pass, hence the requisite area is $(0.125)(0.479)=0.06$ square inches. The diameter at the throat is $0.276 \mathrm{inch}$. 
Similar solution for the exit end gives $x_{2}=0.828, \lambda_{2}=940.0$, and the specific volume at the exit is 143.3 cubic feet. The velocity is therefore 3835 feet per second, the area of the turbine nozzle is 0.672 square inch, and the diameter is $0.924 \mathrm{inch}$.

(b) It is usual not to make allowance for the friction at the throat, since its effect would be small, but to make the nozzle at the throat according to the calculations for a frictionless adiabatic expansion as made above. It will be remembered in justification of this method that the steam discharged through an orifice is practically independent of the pressures, provided only that the exit pressure is less than 0.58 of the initial pressure. (Thus compare Problem 47.)

At the exit end the loss in energy is 15 per cent of $\lambda_{1}-\lambda_{2}$ or 44 B.t. u. The quality at exit for a flow with friction is greater than that for a frictionless flow by an amount equal to the fraction of a pound of steam that this number of B.t.u. would evaporate. Since $r_{2}$ for 2 pounds is 1021.9 , the increase in quality is $\frac{44}{1022}=0.043$. The quality after flow with friction is then $0.828+0.043=0.871$. Hence the specific volume is greater and is $(0.871)(173.1)=150.8$ cubic feet. In order that this turbine nozzle shall deliver the same amount of steam for the same pressure conditions the area at the exit end must be larger than that for the case of frictionless expansion in the ratio of the specific volumes in the two cases. Hence the requisite area is

$$
\frac{150.8}{143.3}(0.672)=0.706 \text { square inch. }
$$

And the diameter is $0.947 \mathrm{inch}$. 


\section{MISCELLANEOUS PROBLEMS}

1. Determine four points on the pressure-volume curve and also four points on the temperature-entropy curve representing the transformation of a pound of dry air undergoing an increase of temperature from $100^{\circ}$ to $200^{\circ}$ Fahrenheit under the following conditions: (a) adiabatically from an initial pressure of 15 pounds, $(b)$ at constant pressure of $15^{1}$ pounds per square inch, $(c)$ at constant volume of 11 cubic feet. Assume a zero of entropy for each initial condition.

2. Plot in the same manner as in Problem 1 the transformation at a constant temperature of one pound of air originally occupying 11 cubic feet at 35 pounds per square inch if the pressure decreases to 15 pounds.

3. Find the work done, the change in internal energy, the change in entropy, and the heat added during the expansion of 3.5 cubic feet of air from 200 to 90 pounds pressure. The temperature is maintained constant at $650^{\circ}$ Fahrenheit absolute.

4. Find the external work done, the change in internal energy, the change in entropy, and the heat added if 3 cubic feet of air at $200^{\circ}$ Fahrenheit expand adiabatically from a pressure of 85 pounds per square inch to a pressure of 15 pounds.

5. A quantity of gas occupying 9 cubic feet at a pressure of 42 pounds per square inch expands until the volume is 14 cubic feet at a pressure of 23 pounds. Find an equation representing a possible expansion between these two points. Find the work done. Find the change in intrinsic energy.

6. A steam engine working between 135 pounds and 2 pounds per square inch pressure requires 225 B.t. u. per indicated horse power per minute. What is the ratio between the thermal efficiency of this engine and that of a Carnot engine working between the same temperature limits?

7. Calculate the work done by an air compressor without clearance in compressing and delivering 10 cubic feet of dry air from an atmospheric pressure of 15 pounds to a gauge pressure of 75 pounds. Assume adiabatic compression.

1 All pressures are absolute and not gauge unless otherwise stated. 
8. What would have been the work in Problem 7 if the compression had been according to the relation $p V^{\mathbf{1 . 3 2}}=$ a constant?

9. If cooling water of temperature $65^{\circ}$ Fahrenheit is available, to what pressure must dry atmospheric air be compressed in order that after cooling it may in an adiabatic expansion fall to $32^{\circ}$ Fahrenheit? The initial temperature of the air is $70^{\circ}$ Fahrenheit. Atmospheric pressure is 15 pounds per square inch.

10. Find from the entropy chart of page 86 what the pressure is upon a mixture of steam and water one pound of which occupies 6 cubic feet, if the quality is 95 per cent.

11. What is the quality of a mixture of steam and water of entropy 1.590 if one pound occupies 8 cubic feet?

12. What is the quality in Problem 11 if the mixture occupies 3 cubic feet?

13. One pound of saturated steam at a pressure of 140 pounds per square inch undergoes a constant heat content change until the final pressure is 25 pounds. Find the quality. Calculate the final intrinsic energy.

14. Steam at 140 pounds gauge and superheated $100^{\circ}$ Fahrenheit expands adiabatically. What is the pressure when the steam is dry saturated steam? Atmospheric pressure is 15 pounds.

15. If the steam of Problem 14 expands in an engine cylinder to five times its original volume, what, approximately, are the final pressure and quality?

16. Steam of entropy 1.560 and heat contents 1200 B.t.u. expands adiabatically until $200 \mathrm{~B}$.t. u. have been converted into external work. Find the final quality.

17. Find from the entropy chart of page 86 the limit of a throttling calorimeter when used to measure quality in steam of pressure 145 pounds if it is connected to the atmosphere of which the absolute pressure is 14.7 pounds.

18. Steam of quality 99 per cent and pressure 160 pounds passes through a reducing valve. To what pressure must it be throttled in order that the steam discharged shall be dry and saturated?

19. Steam from a boiler is passed into a barrel calorimeter in which there is 200 pounds of water at a temperature of $60^{\circ}$ Fahrenheit. When 3 pounds have been condensed, the final temperature is $75^{\circ}$ Fahrenheit. The boiler pressure is 145 pounds. Find the quality. 
20. Feed water of temperature $80^{\circ}$ Fahrenheit enters a boiler and passes from a boiler through a superheater. It emerges at 100 pounds pressure and a temperature of $600^{\circ}$ Fahrenheit. If 8 pounds of water is thus converted into steam for each pound of coal burned, find the equivalent evaporation from and at $212^{\circ}$ Fahrenheit.

21. Find from values taken from the entropy chart of page 86 the Rankine efficiency of an engine working between 140 pounds with a quality of 95 per cent and 10 pounds.

22. A four-stage impulse turbine is designed to develop equal velocity in each of the stages. If supplied with steam of 100 pounds pressure and $100^{\circ}$ Fahrenheit superheat and expanding to a condenser pressure of 2 pounds, find the velocity developed in each stage.

23. What is the area at the throat of a nozzle which allows 50 pounds of steam to pass in a minute, if the entering steam is 150 pounds pressure and $100^{\circ}$ Fahrenheit superheat, and the back pressure is less than 0.58 of the admission pressure?

24. What would be the area if the back pressure in Problem 23 were 100 pounds?

25. How much work is done in compressing adiabatically one pound of a mixture of steam and water of quality 80 per cent and pressure $\mathbf{1 4 . 7}$ pounds until the volume is 6 cubic feet?

26. Two pipes deliver into a third. One supplies 300 gallons per minute at a temperature of $80^{\circ}$ Fahrenheit and the other supplies steam of quality 75 per cent and pressure 100 pounds per square inch at the rate of 5 pounds per minute. What is the resulting quality of the mixture in the third pipe?

27. A hot-water heater is constructed on the above principle (Problem 26). Ten gallons of water at $200^{\circ}$ are to be obtained per minute in the third pipe. The entering water is at $60^{\circ}$ Fahrenheit. The entering steam is at 50 pounds pressure and dry. What is the smallest amount of steam in pounds required per minute?

28. What approximately is the final temperature and pressure exerted by a mixture of steam and water in a closed tank if the tank originally contained 10 pounds at a pressure of 140 pounds per square inch and a quality of 98 per cent and 9000 B.t. u. are removed by cooling? 
29. Find the increase in volume per pound caused by passing dry steam at a pressure of 130 pounds per square inch through a superheater which raises the temperature to $500^{\circ}$. Fahrenheit.

30. A throttle valve reduces the pressure of dry steam from 135 pounds to 100 pounds. Find the loss in availability in B.t.u. per pound if the lowest available temperature is $141.5^{\circ}$ Fahrenheit.

31. Plot to scale the indicator card for a theoretically perfect engine which has a stroke of 30 inches, a clearance of 5 per cent, cut-off at 30 per cent, compression at 5 per cent, release at the end of the stroke, a piston diameter of 16 inches, and makes 100 revolutions per minute. If the entering steam is 100 pounds pressure, the condenser pressure is 2 pounds, and the expansion and compression may be assumed to be adiabatic, find the work done during admission, expansion, exhaust, and compression. Find the indicated horse power. Find the mean effective pressure. The engine is of course double-acting, having valves at each end of the cylinder.

32. Find the horse power of a hot-air engine which works on a Sterling cycle from a maximum temperature of $700^{\circ}$ Fahrenheit and a maximum pressure of 110 pounds absolute, if the volume of the working cylinder is 2 cubic feet and that of the displacement cylinder is 6.5 cubic feet. Neglect clearance. The lowest available temperature is $90^{\circ}$ Fahrenheit. The engine makes 100 revolutions per minute.

33. Draw to scale the cross section of a nozzle which will discharge 0.50 pound of steam per second for frictionless adiabatic expansion from 120 pounds pressure and $120^{\circ}$ Fahrenheit superheat to 2 pounds pressure per square inch.

34. If the friction loss in the nozzle of Problem 33 is to be taken as 12 per cent and the nozzle is drawn tapered from the throat to the exit end, how much larger must be the diameter of the exit end in order that the same amount of steam shall pass per second?

35. If the steam of Problem 33 had not been superheated but had been of quality 98 per cent, what would have been the velocity at the exit end of the nozzle?

36. If a steam turbine is to have four stages of equal velocity and to be supplied with steam under the conditions of Problem 33, what will be the pressures in the intermediate stages? 



\section{TABLES}

TABLE 1

\begin{tabular}{|c|c|c|c|c|c|c|c|}
\hline \multicolumn{4}{|c|}{$\mathrm{G}_{\Delta \mathrm{S}}$} & \multirow{2}{*}{$\begin{array}{c}\begin{array}{c}\text { EXPANSION } \\
\text { COEFFICIENT } a\end{array} \\
0.0036600\end{array}$} & \multirow{2}{*}{$\begin{array}{c}\begin{array}{c}\text { Pressure } \\
\text { Cokfficient } \beta\end{array} \\
0.0036626\end{array}$} & \multirow{2}{*}{$\begin{array}{l}\text { Observer } \\
\text { P. Chappuis }\end{array}$} & \multirow{2}{*}{$\begin{array}{l}\text { Date } \\
1887\end{array}$} \\
\hline Hydrogen & . & . & . & & & & \\
\hline Hydrogen & . . & 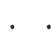 & 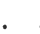 & 0.003661 & 0.003668 & Regnault & 1840 \\
\hline Air. . & . . & 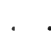 & . & 0.003670 & 0.003665 & Regnault & 1840 \\
\hline Air. . & . . & 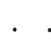 & & - - - & 0.003663 & Kuenen and Randall & 1895 \\
\hline Carbon dic & xide (C & $\mathrm{CO}_{2}$ & & 0.003710 & 0.003688 & Regnault & 1840 \\
\hline Nitrous ox & ide $\left(\mathrm{N}_{2}\right.$ & $\left.{ }_{2} \mathrm{O}\right)$ & & 0.003719 & 0.003676 & Regnault & 1840 \\
\hline Oxygen . & . . & . & . & -5 & 0.003674 & von Jolly & 1874 \\
\hline Nitrogen & . . & . & . & - & 0.003667 & von Jolly & 1874 \\
\hline Argon & . . & . & . & & 0.003668 & Kuenen and Randall & 1895 \\
\hline Helium . & . . & . & . & - & 0.0036627 & Travers and Jaquerod & 1903 \\
\hline
\end{tabular}

TABLE 2

\begin{tabular}{|c|c|c|c|c|}
\hline Substaxce & $\begin{array}{c}\text { SPECIFIC Volume } \\
\text { Cubic feet per pound }\end{array}$ & $\begin{array}{l}\text { SPECific Heat } \\
\text { Constant pressure }\end{array}$ & $\begin{array}{l}\text { Specific Heat } \\
\text { Cónstant Volume }\end{array}$ & $\begin{array}{l}\text { RूTाO } \\
k=\frac{c_{p}}{c_{v}}\end{array}$ \\
\hline Hydrogen . . & 178.2 & 3.409 & 2.406 & 1.408 \\
\hline Oxygen . . . & 12.21 & 0.2175 & 0.155 & 1.403 \\
\hline Air (dry). . . & 12.39 & 0.2375 & 0.1689 & 1.401 \\
\hline Nitrogen . . . & 12.75 & 0.2438 & 0.173 & 1.409 \\
\hline Marsh gas $\left(\mathrm{CII}_{4}\right)$. & 23.30 & 0.593 & 0.467 & 1.31 \\
\hline Carbon dioxide . & 8.10 & 0.2025 & 0.171 & 1.30 \\
\hline Carbon monoxide. & 12.80 & 0.2426 & 0.173 & 1.401 \\
\hline Ethlyene $\left(\mathrm{C}_{2} \mathrm{H}_{4}\right)$. & 12.58 & 0.404 & 0.332 & 1.24 \\
\hline
\end{tabular}

1 For standard conditions of $p=14.7$ pounds per square inch and $t=32^{\circ}$ Fahrenheit. 
TABLE $3^{1}$

(F.P.S. Gravitational system; degrees Fahrenheit, British thermal units)

Dry Saturated Steam

\begin{tabular}{|c|c|c|c|c|c|c|c|c|c|c|c|}
\hline 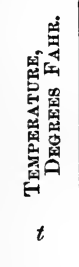 & 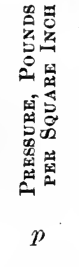 & 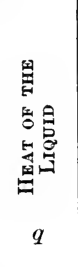 & 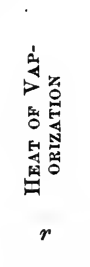 & 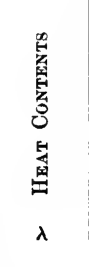 & 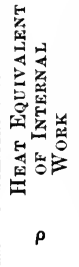 & 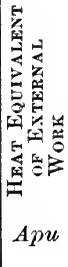 & 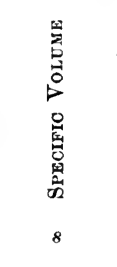 & 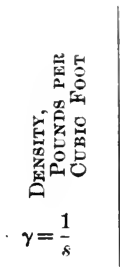 & 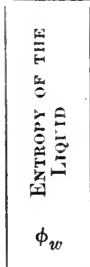 & 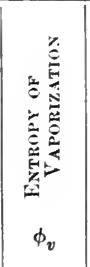 & 要 \\
\hline & & 28.1 & 57.0 & 85.1 & 9.8 & 57.2 & 7. & 000828 & 0.0 & 0 & \\
\hline & & 33.1 & 54.4 & 87.5 & 66.7 & 57.7 & & & & & \\
\hline & 10 & 38.1 & 51.8 & 89.9 & 3.6 & 58.2 & 868. & 01152 & 0.0747 & & \\
\hline & 429 & 43.1 & 49.2 & 92.3 & 90.5 & 58.7 & & & & & \\
\hline & 506 & 48.1 & 46.5 & 94.6 & 37.2 & 59.3 & 634. & 01577 & 0.0984 & & \\
\hline & & 53.1 & & 97.0 & 34.1 & & & & & & . \\
\hline & 696 & 58.1 & 41.2 & 99.3 & 30.9 & 60.3 & 46 & 2131 & 0.1 & & \\
\hline & & 68.0 & & & 4.4 & & & & & & \\
\hline 101.8 & & 69.8 & 7 & 5 & 73.1 & 61.6 & & & & & \\
\hline 110 & 1.271 & 78.0 & & & 67.7 & & & & & & \\
\hline & 1.689 & 88 & & & 0 & 63.4 & & & & & \\
\hline 126. & & 94.2 & & & & & & & & & \\
\hline & & 6 & & & 4 & & & & & & \\
\hline & & 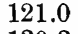 & & & 6 & & & & & & 6 \\
\hline & & 3 & & & .1 & & & & 0. & & \\
\hline & & & & & .8 & & & & & & \\
\hline & & & & & & 7. & & & & & \\
\hline & l.7 & 1 & & & & & & & & & \\
\hline & & & & & & & & & & & \\
\hline & & 2 & 9 & & & 76 & 13 & & & 1 & \\
\hline & & & & & & & & & & & \\
\hline & & & & & & & & & & & \\
\hline & 8 & & & & & & & & & & \\
\hline & 85 & 28 & 8. & & & & & & & & 1 \\
\hline & & & & & & & & & & & \\
\hline & & & & & & & & & & & \\
\hline & & & & & & & & & & & \\
\hline & & & & & & & & & & & \\
\hline & & & & & & & & & & & \\
\hline & & & & & & 82 & & & & & \\
\hline & & & & & & & & & & & \\
\hline & & & & & & & & & & & \\
\hline & & & & & & & & & & & \\
\hline & & & & & & & & & & & \\
\hline & & & & & & & & & & & \\
\hline & & & & & & & & & & & \\
\hline & & & & & & & & & & & \\
\hline & & & & & & & & & & & \\
\hline & & & & & & & & & & & \\
\hline & & & & & & & & & & & \\
\hline & & & & & & & & & & & 1.5 \\
\hline & & & & & & & & 0.54 & 0.56 & 0.960 & 1.5 \\
\hline
\end{tabular}

1 These values are taken from the 1909 edition of Professor C. H. Peabody's "Tables of the Properties of Steam," with the permission of the author and the publishers, Wiley and Sons. 
LOGARITHMS

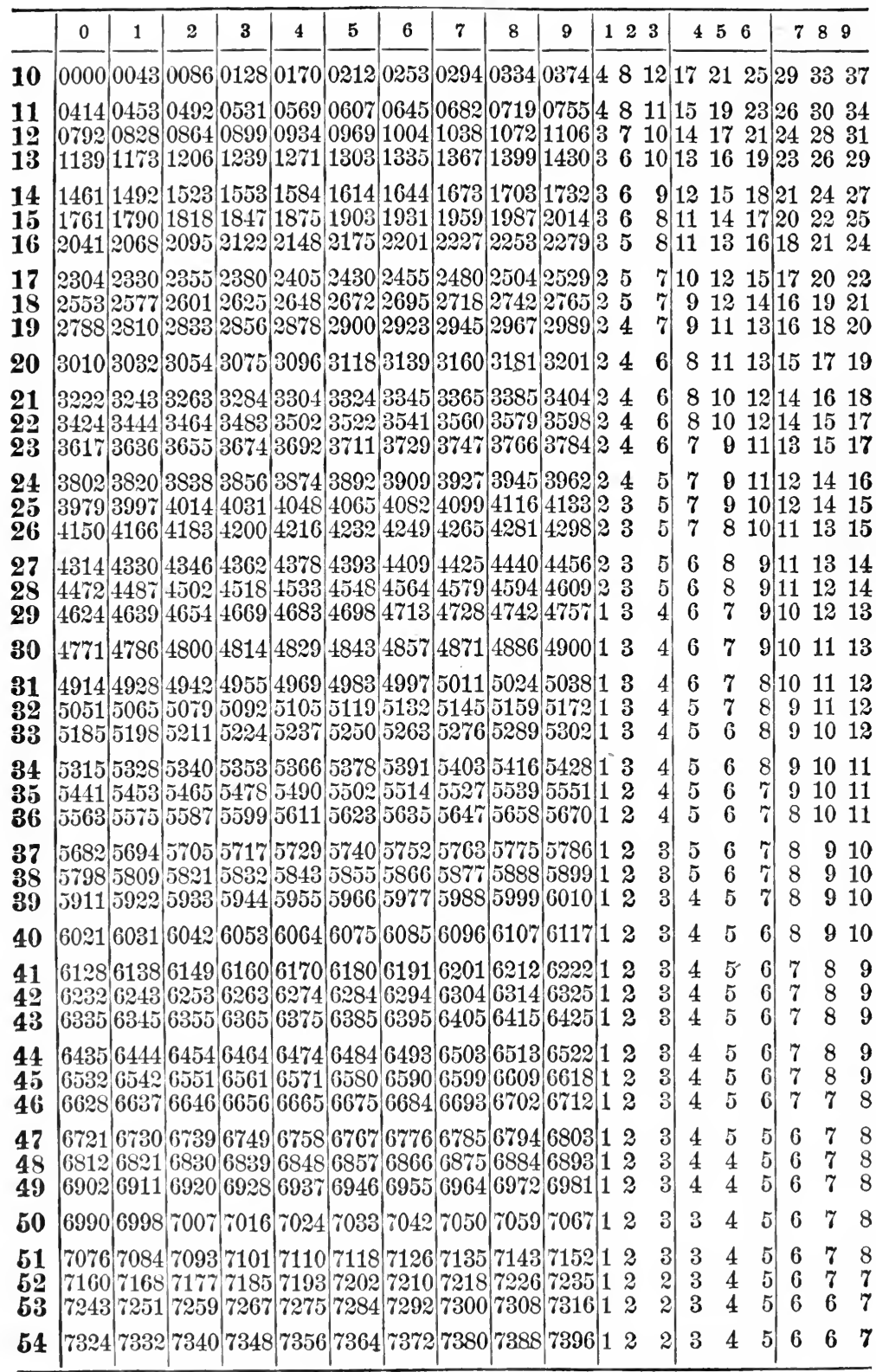


LOGARITHMS

\begin{tabular}{|c|c|c|c|c|c|c|c|c|c|c|c|c|c|}
\hline & & & & 3 & 4 & & & & & 9 & $\begin{array}{lll}1 & 2 & 3\end{array}$ & $\begin{array}{lll}4 & 5 & 6\end{array}$ & $\begin{array}{lll}7 & 8 & 9\end{array}$ \\
\hline & 04 & 7412 & 7419 & 7427 & 7435 & 7443 & 7451 & 7459 & 7466 & 7474 & 129 & & 56 \\
\hline & 599 & $\begin{array}{l}7490 \\
7566 \\
7642\end{array}$ & & & $\begin{array}{l}7010 \\
7589 \\
7664\end{array}$ & $\begin{array}{l}7520 \\
7597 \\
7672\end{array}$ & $\begin{array}{l}7528 \\
7604 \\
7679\end{array}$ & $\begin{array}{l}7536 \\
7612 \\
7686\end{array}$ & & & & & \\
\hline $\begin{array}{l}9 \\
0 \\
1\end{array}$ & & $\begin{array}{l}7716 \\
7789 \\
7860\end{array}$ & & & $\begin{array}{l}y \\
7 \\
r\end{array}$ & $\begin{array}{l}7745 \\
7818 \\
7889\end{array}$ & & & & & & & \\
\hline & & 31 & & & & 59 & $\mid 7966$ & 73 & & & & & \\
\hline & & & & & & & & & & & & & \\
\hline & 62 & 69 & 75 & & 8089 & 8096 & 8102 & 8109 & 8116 & 8 & & & \\
\hline & 29 & 36 & 142 & 8149 & 8156 & 8162 & 8169 & 8176 & 8182 & 8189 & 12 & 33 & 55 \\
\hline & 95 & 02 & 09 & 15 & 8222 & 28 & 8235 & 8241 & 48 & 54 & & & \\
\hline & & 31 & & & & 93 & 99 & & & & & & \\
\hline & & 81 & & & 8351 & $835 \%$ & 8363 & 8370 & 86 & 82 & & & \\
\hline & 51 & & & & & $\begin{array}{l}20 \\
82\end{array}$ & 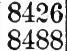 & & & & & & \\
\hline & 13 & 19 & 5. & 31 & 37 & 43 & 8549 & 8555 & 8561 & 67 & & & \\
\hline & & 79 & & & 8597 & 303 & 09 & 15 & & & 2 & & \\
\hline & 92 & 98 & 04 & 10 & 16 & 722 & 8727 & & 39 & 745 & $\begin{array}{lll}1 & 12\end{array}$ & & \\
\hline & 51 & 456 & 8762 & 68 & 8774 & 8779 & 8785 & 8791 & 8797 & 8802 & 12 & 3 & 4 \\
\hline & & 14 & 20 & 55 & 31 & 37 & 342 & 18 & & 59 & 1 ? & & \\
\hline & & & & & & & & & & & & & \\
\hline & & & & & & & & & & & & & \\
\hline & & & & & & & & & & & & & \\
\hline & 85 & 90 & 096 & & 06 & 2 & 117 & & & & & & \\
\hline & 8 & & & & & & 70 & & & & & & \\
\hline & & & & & & & & & & & & & \\
\hline & & & & & & 69 & & & & & & & \\
\hline & & & 0 & 09 & 9 & 9320 & 93 & 9330 & 35 & 9340 & 112 & & 44 \\
\hline & & & & & $136 \pi$ & 370 & 9375 & 9380 & & & & & \\
\hline & & & & & & & & & & & & & \\
\hline & & & & & & 69 & & & & & $\begin{array}{lll}1 & 1\end{array}$ & & \\
\hline & & & 504 & & 3 & 8 & 23 & 528 & & & & & \\
\hline & & & & & & & & & & & & & \\
\hline & & & & & 509 & 614 & & & & & & & \\
\hline & & & & & 96 & 61 & 96 & 771 & & & & & \\
\hline & & & & & & & & & & & & & \\
\hline & & & & & 97 & 9800 & 980 & 9809 & 9814 & 9818 & $\begin{array}{lll}0 & 1 & 1\end{array}$ & & 34. \\
\hline & & & & & & & & & & & & & \\
\hline & & & & & & & & & & & & & \\
\hline & & & & & & & 9939 & & & & & & \\
\hline 19 & 956 & 61 & 965 & 969 & 974 & 97 & 9983 & 987 & 991 & 996 & & & \\
\hline
\end{tabular}





\section{INDEX}

Absolute pressure, 46

Absolute temperature, 12, 14

Absolute zero, 13

Adiabatic, for gases, $16,19,20,32$; for steam, 71 ; for superheated steam, 97

Air compressor, 53

Air flow, 113

Air refrigeration, 57

Availability of energy, 2, 121

Battelli, 93

Boiler explosion, 77

Boiler horse power, 78

Boulvin diagram, 87

Boyle's law, 8

British thermal unit, 5

Calorie, 5

Calorimeter, throttling, 119, 121

Carnot cycle, 22 ; for perfect gas, 30 ; plot of, 34, 35; efficiency, 24, 31; engine, 23 ; theorem, 24, 26

\section{Cazin, 71}

Centigrade scale, 4, 12

Chappuis, 12

Charles's law, 11

Clausius, 27 ; inequality of, 37

Clearance, in air compressor, 53 ; in gas engine, 54

Compression, steam at, 88

Condenser, jet, 80 ; surface, 79

Conservation of energy, 2, 5

Continuity, equation of, 105

Cycle, see Carnot, reversible, etc.

Cylinder condensation, 90, 98

Dalton's law, 44

De Laval turbine, 122

De Rochas gas-engine cycle, 54

De Saint Venant, formula of, 104

Diesel internal-combustion cycle, 56

Dry steam, 62
Efficiency, see Carnot, Rankine, etc.

Energy, 1; see intrinsic, kinetic, etc.

Entropy, 31; measurement of, 3:3 ; for gases, 41 ; for water, 67 ; for steam, 68 ; for superheated steam, 94

Equations, résumé of, for gases, 44 ; for steam, 74 ; for superheated steam, 99 ; for flow of fluids, 114

Equivalent evaporation, 78

Fahrenheit scale, 4

First law of thermodynamics, 5

Fliegner's formulas, 113

Flow of fluids, Chap. V

Friction in flow of fluids, 109

Gases, molecular theory of, 3 ; laws of, 8-11; general equation, 14 ; perfect, 10, 38, 40; imperfect, 44; see also intrinsic energy, entropy, specific heat, etc.

Gay-Lussac, law of, 11

Heat contents, see heat equations

Heat equations, for gases, 40 ; for steam, 65, 67; for superheated steam, 94

Helium, 13

Hirn, 71

Hydrogen, 13

Hyperbolic expansion line, 91

Imperfect gas, 44

Incomplete steam expansion, 81

Indicator, 18 ; card, 88

Injector, 118

Intrinsic energy, 2, 16; for gases, 43 ; for steam, 67 ; for superheated steam, 96

Irreversible process, 35, 37, 109

Isentropic, 16, 32, 33; for gases, 41; for steam, 71 ; for superheated steam, 97 
Isoenergic, 16 ; see also intrinsic enérgy

Isothermal, 16, 19, 20

Joule, 3, 5; law of, 9 ; experiment of, 10

Kelvin, 28, 121

Kinetic energy, 2 ; see molecular kinetic theory

Kinetic energy due to expansion, 104, $109,114,115$

Knohlausch and Jakob, 93

Knoblausch, Linde, and Klebe, 93

Latent heat, 7 ; internal, 7 ; external, 7 ; of vaporization, 61

Lewicki, 99

Loss of kinetic energy due to friction, $109,116,122$

Mechanical equivalent of heat, 5

Molecular kinetic theory, 3, 8, 11, 13, $14,61,65,92$

Mollier diagram, 122

Motivity, 121

n, for gases, 39, 43 ; for steam, 107

Napier's formulas, 112

Newton, 1, 21

Nozzle area, 122

Otto gas-engine cycle, 54

Peabody, 119

Perfect gas, see gases

Polytropic transformation, 39,47

Porous plug experiment, 35

Potential energy, 2

Pressure, defined, 1.5 ; absolute, 46

Pressure, coefficient, 11

Pressure-entropy plot, 96, 120

Pressure-volume plot, 16, 17

Problems, see C'ontents

Quality, 66, 96, 119

Rankine, 65, 112; Rankine cycle and efficiency, 80)
Regenerative cycle, 51

Regnault, 12, 14, 73

Reversible cycle, 24

Second law of thermodynamics, 21, 26

Solutions of problems, see Contents

Specific, use of the term, 14

Specific heat, 5 ; of gases, 15,42 ; of water, $6: 3$; of steam, 73 ; of superheated steam, 9:3

Specific volume, of water, 65 ; of steam, 65,67 ; of superheated steam, 94

Steam, see under entropy, quality, etc.

Sterling hot-air engine, 51

Stodola, 99

Superheated steam, 93; see under entropy, kinetic energy, etc.

Superheating in practice, 98, 102

Temperature, 3, 12, 14, 28

Temperature-entropy plot, 33; for steam, 68; for superheated steam, 94 ; use of, 86,122

Thermal units, 4

Thermodynamic scale of temperature, 28

Thermometer, mercury, 3 ; hydrogen, 12,14

Throttle control, 121

Throttling calorimeter, 119, 121

Transformation, see adiabatic, isother-

- mal, etc.

Tumlirz, 93

Turbine nozzle, 112, 122

Vapor, see steam

Vaporization, 61

Volume, see specific volume

Volume coefficient, 11

Water, specific heat, 63 ; specific volume, 65

Water vapor, see steam

Wet steam, 62

Work 1 ; calculations for, 17

Zeuner, formulas of, 65, 107, 108 
\begin{tabular}{|c|}
\hline+ \\
$\cdot$,
\end{tabular}

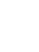


1 


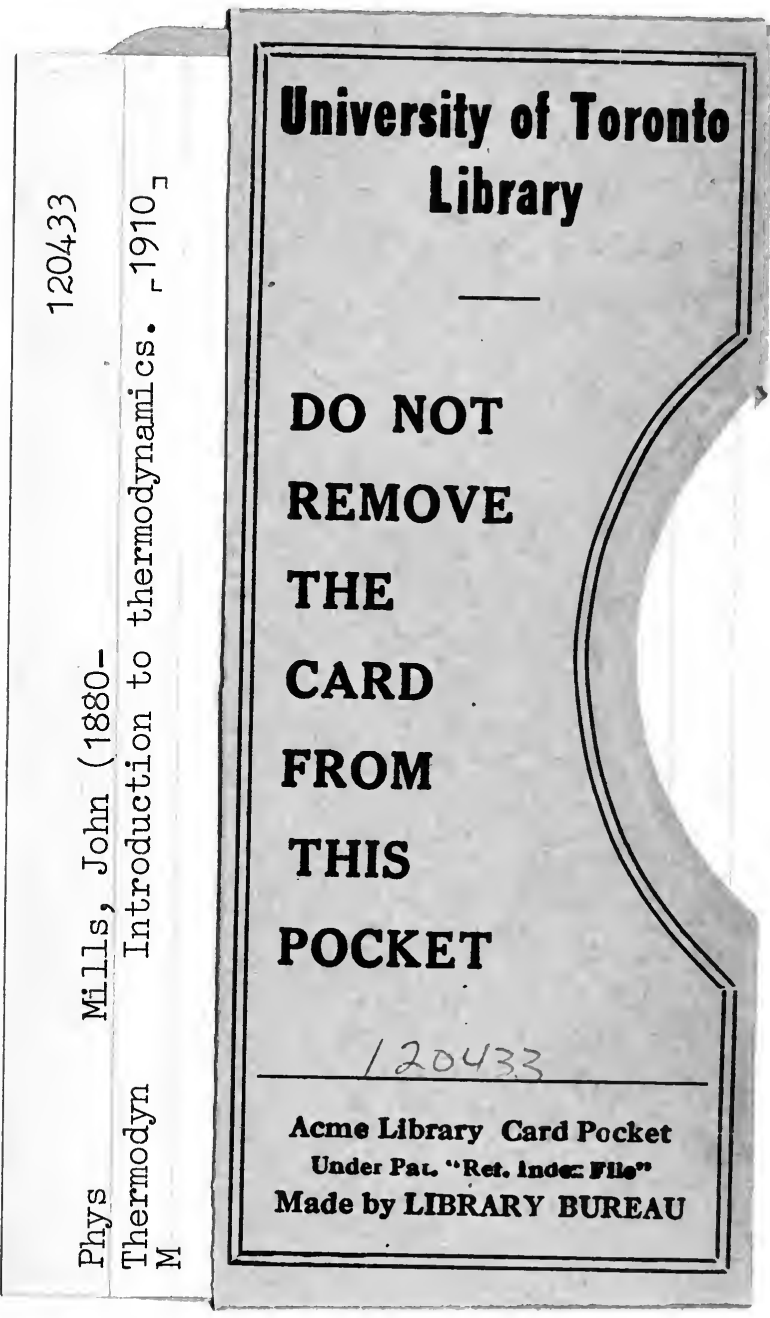


-

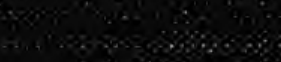

